\title{
595.76 CI6c
}

W 


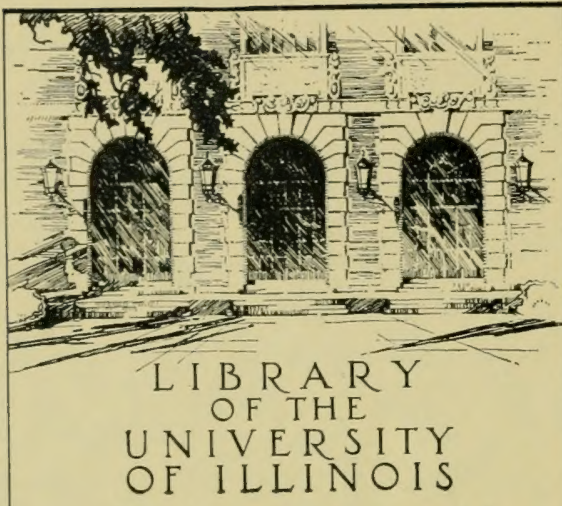

From the Library of Professor Henry F. Wickham University of Iowa Presented in 1942

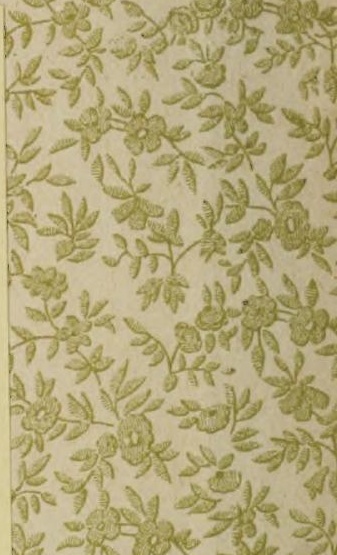

if

\section{NATURAL}

\section{DEC 21942 HISTORY}

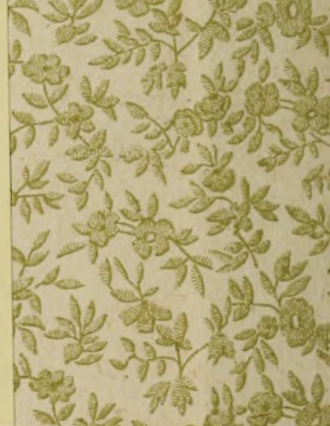

+ 1 1) in 19 ofre

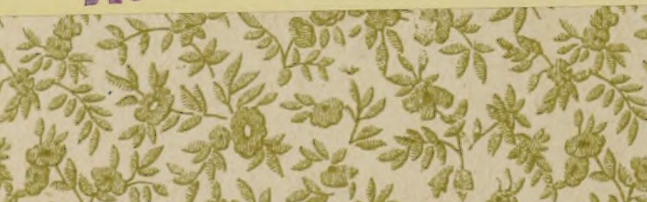




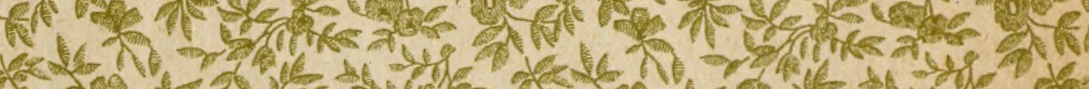
Tfe - Th a $65^{2}$ -

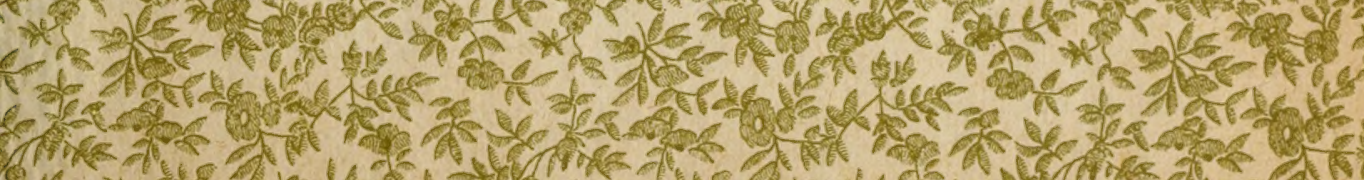


Digitized by the Internet Archive in 2010 with funding from

University of Illinois Urbana-Champaign 




\title{
GATALOGUE MÉTHODIQUE
}

\author{
DES
}

ELATÉRIDES

CONNUS EN 1890

PAR

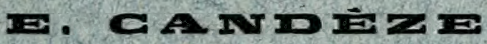

Docteur en Médecine,

Membre de l'Académie des Sciences, Lettres et Beaux-Arts de Belgique, de l'Académie des Sciences de Philadelphie,

Membre honoraire de la Saciété Entomologique de France, etc., etc.

\section{LIÉGE}

IMPRIMERIE H. VAILLANT-CARMANNE

Rue St-Adalhert, 8. 


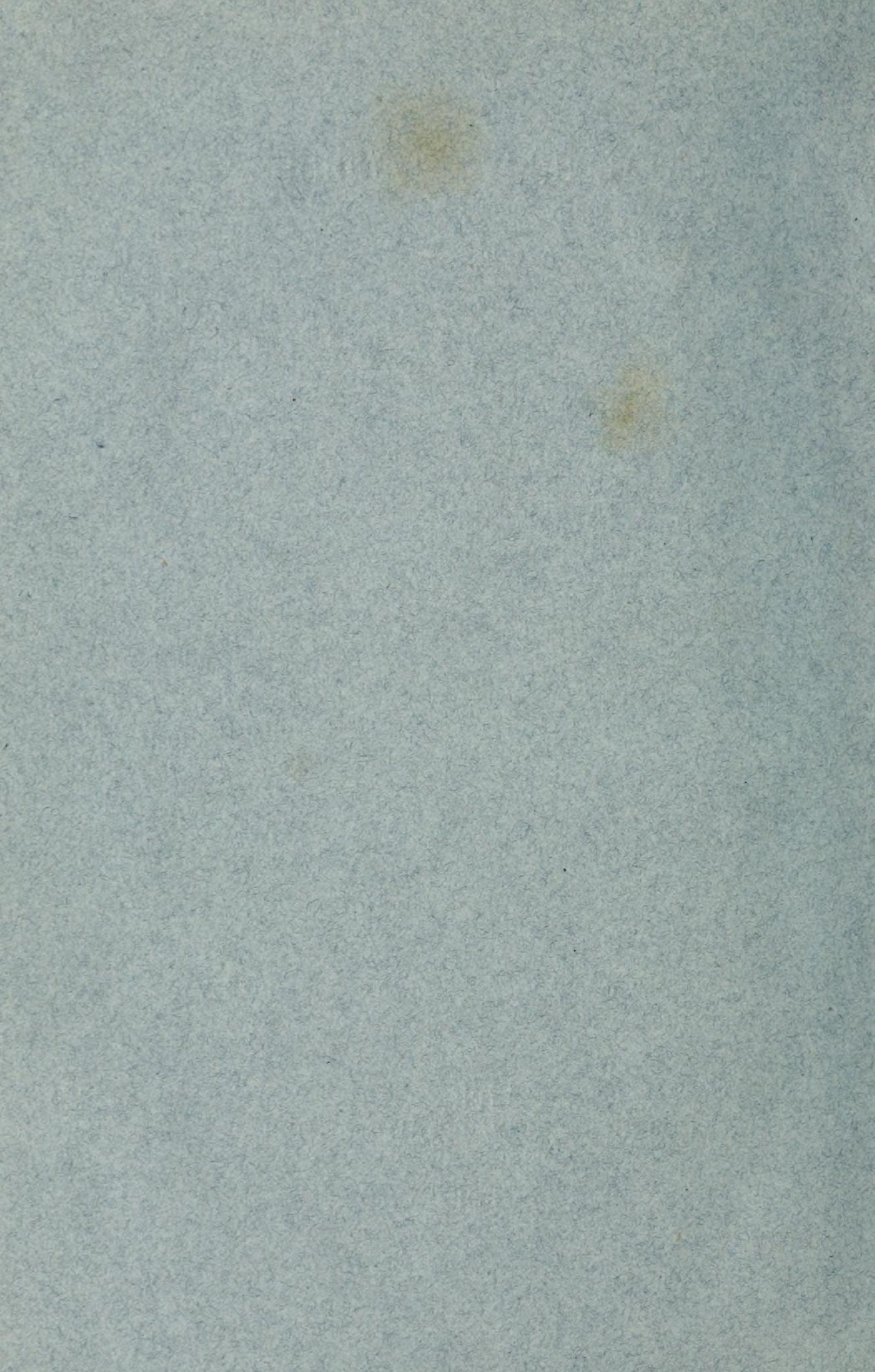


CATALOGUE MÉTHODIQUE DES ÉLATÉRIDES 



\section{GATALOGUE MÉTHODIQUE}

DES

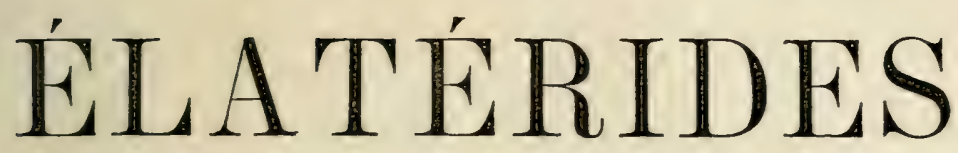

CONNUS EN 1890

PAll

\section{E. CA}

Docteur en Mrédecine,

Membre de l'Académie des Sciences, Lettres et Beaux-Arts de Belgique, de l'Académie des Séiences de Philadelphie,

Membre honoraire de la Société Entomologique de France, etc., etc.

\section{LIÉGE}

IMPRIMERIE H. VAILLANT-CARMANNE

Rue St-Adalhert, 8 . 



\section{AVAN'T-PROPOS}

La nécessité des catalogues s'impose de plus en plus, en présence du flot montant des espèces de plantes et d'animaux que l'on décrit partout.

Se crois inutile de développer cette proposition qui ralliera, j'en suis certain, l'avis de tous les naturalistes qui se sont attachés à l'étude systématique des nombreux spécimens de plantes et d'insectes qui s'accumulent dans les Musées publics et particuliers.

Nous sommes loin du temps de Linnée. Le naturaliste suédois osait entreprendre, et mener à bien, la classification de tous les êtres vivants. Ses disciples d'aujourd'hui, plus modestes, s'attachent à l'étude d'une famille, d'une tribu, d'un genre même, et s'y cantonnent.

Et malgré cette spécialisation (que l'on me pardonne cette expression), la mise en ordre de telle famille, de telle tribu, de tel genre n'est pas toujour's chose aisée.

Pour ne parler que des insectes, ou plutôt des coléoptères, il est bien des genres qui réclament impérieusement un arrangement systématique et cet arrangement n'arrive pas et menace de n'arriver jamais.

Je citerai comme exemple le genre Onthophagus. Où est l'entomologiste qui aurait le courage d'entreprendre la monographie de ces insectes? Harold et Van Lansberge, qui en avaient fait une étude spéciale, ont reculé devant un travail d'ensemble. Il ne s'agit cependant que d'un genre. 
La raison en est facile à comprendre. Les types des espèces décrites, au nombre de plusieurs centaines, sont dispersés dans un nombre considérable de Musées et de collections particulières, en Europe, en Amérique, en Afrique et jusqu'en Australie. Le monographe consciencieux se trouverait probablement arrêté au début de son travail, les Musées en général se refusant à communiquer leurs types, par l'impossibilité d'examiner les espèces décrites, aux lieux où elles se trônvent. Les voyages de circumnarigation ne sont pas à la portée de tout le monde, et le temps n'est pourtant pas éloigné où une bonne monographie demandera à son auteur un tour du monde préalable.

Un catalogue disposé alphabétiquement est un travail purement bibliographique. Une bonne bibliothèque suffit, à la rigueur. Il en est tout autrement d'un catalogue méthodique.

Ce n'est pas sans appréhension que je publie le présent catalogue. Les Elatérides y sont disposés, autant que possible, dans l'ordre de leurs affinités et il eît été bon que j'eusse pu les examiner tous. Or, l'on me croira sans peine lorsque j'avouerai que je n'ai pu toujours le faire.

Il est tel Musée important, en Europe, où sont conservés des types de genres et d'espèces que j’aurais voulu examiner, dans l'intérêt de mon Catalogue. Je n'ai pu les voir. Ils étaient introuvables, en dépit de la bonne volonté, - à laquelle je me plais à rendre hommage - des personnes préposées à leur conservation.

Force m'a été de passer outre.

Le classement méthodique est néanmoins possible lorsque les auteurs ont en soin d'indiquer les affinités des espèces qu'ils décrivaient; mais il n'en est point toujours ainsi. Nombre d'espèces de l'Australie et de la Nouvelle Zélande, par exemple, ont été dénommées et sommairement décrites sans ces renseignements indispensables. 
Cette impossibilité où je me suis trouvé de contrôler et classer les espèces en question sera indiquée chaque fois qu'il y aura lieu.

A l'époque où Lacordaire a publié le quatrième volume du Généra des Coléoptères et lorsque moi-même j'ai entrepris la Monographie des Elatérides, on connaissait 65 genres et environ un millier d'espèces.

La famille s'est accrue depuis.

Le catalogue de Munich renseigne 174 genres, dont plusieurs sont tombés en synonymie ou appartiennent à d'autres familles, et 2693 espèces.

Le catalogue ci-après énumère 238 genres et 3960 espèces. Il me paraît que ces chiffres seuls en démontrent l'utilité sans qu'il soit nécessaire d'en justifier la publication par d'autres raisons.

J'y ai introduit la répartition géographique pour certains genres devenus très riches en espèces, et cosmopolites.

Les grandes divisions de Wallace m'ont servi de base. Je crois utile de rappeler ici, brièvement, les six régions admises par le naturaliste anglais, ce sont: $1^{\circ}$ la région palécur;tique, comprenant l'Europe, le pourtour de la Méditerranée, le nord de l'Asie jusqu'au pied des grandes montagnes du centre; $2^{\circ}$ la région éthiopique, embrassant l'Afrique au delà des grands déserts, l'Arabie méridionale et les îles africaines; $3^{\circ}$ la région orientale, comprenant l'Asie au sud de l'Himalaya, Ceylan, les grandes îles Malaises jusqu'à Timor; $4^{\circ}$ la région australienne, composée de l'Australasie, de la Polynésie et de la Micronésie; $5^{\circ}$ la région néotropique, qui correspond à l'Amérique du Sud, et . $6^{\circ}$ la région néarctique, qui embrasse l'Amérique du Nord et ses îles.

Il va de soi que ces divisions géographiques ne sont pas absolument rigoureuses, les parties limitrophes offrant des mélanges nombreux, là où elles ne sont pas constituées par de vastes mers ou de hautes montagnes. 
Ainsi la $1^{\text {rc }}$ et la $3^{\circ}$ région se fondent l'une dans l'autre en Chine et an Japon. La $1^{\text {re }}$ et la $2^{3}$ ont des limites mal définies au nord de l'Arabie, en Perse et dans l'Afghanistan. Les deux dernières ont leur trait d'union dans l'Amérique centrile où se rencontrent des espèces mexicaines et colombiennes sans qu'il soit possihle d'établir une ligne de démarcation entre les deux régions, que l'on trouvera nettement distinctes, néanmoins, si l'on compare les faunes des régions plus écartées au Nord et au Sud.

Enfin certains pays comme Madagascar, la Nouvelle Zélande, le Chili, possèdent une faune entomologique spéciale ou qui se rattache, par quelques points, à d'autres faunes parfois fort éloignées. La grande île africaine a une indépendance semblable à celle d'un vaste continent. D'autre part, les régions arctiques des deux mondes ont une foule d'espèces communes.

Quoi qu'il en soit de ces exceptions, la distribution géographique des insectes a une importance très réelle pour le plus grand nombre, et il convient d'en faire grand cas dans la caractéristique des genres ou des espèces.

L'indication de patrie sera forcément un peu brève; mais qu'on ne perde pas de vue qu'il ne s'agit ici que d'un catalogue et que les mentions plus détaillées sont mieux à leur place dans les ouvrages où les espèces sont décrites. Je citerai comme exemple le Melanoxanthus melanocephatus. Cette espèce se rencontre, sous les tropiques, en Asie, en Afrique et en Amérique et la mention de l'habitat demanderait plusieurs lignes. Le catalogue se borne à clire: "Zone intertropicale. .: Quant aux indications plus détaillées, elles ont été faites ailleurs. Ainsi du Mrvistlus scobrimula, etc. Diantres telles que celles du Laron sinensi: ont été rectifiées. Cr rernier a été décrit r'après des exemplaires provenant de Chine et nommé en conséquence. Depuis lors, j'ai pu me convaincre que l'espèce se trouve également dans l'Hin- 
doustan, dans l'Inde transgangétique, y compris la presqu'ile de Malacca, à Sumatra, à Bornéo, etc., ce qui a été relevé dans d'autres publications. Ici je dois me borner à une désignation sommaire.

On m'a fait observer aussi qu'il eût été bon de faire connaître où se trouvent les types de chaque espèce; à quoi je répondrai que l'indication en a été faite pour toutes les espèces que j'ai décrites, à la suite de leurs diagnoses, et que l'absence de mention spéciale de ce genre signifie que le type existe dans ma propre collection; je ne puis, naturellement, rien dire à cet égard de celles qui n'ont pas été décrites par moi, et que souvent je n'ai même pas vues.

Reste la question de la nomenclature. J'ai fait ma profession de foi il y a longtemps déjà, dans la préface de la Révision de la Monographie des Elatérides. Je n'ai pas à y revenir ici.

Toutefois cette importante question ayant fait ultérieurement l'objet de plusieurs Mémoires et tout récemment encore ayant donné lieu, à Paris, non seulement à de nombreuses déclarations, mais en outre à l'élaboration d'un " projet de loi ${ }_{n}$ scientifique, je ne puis me dispenser d'ajouter, dans cet avant-propos, quelques mots de confirmation à la manière de voir qui m'a toujours servi de règle, en matière de nomenclature.

Ennemi des mesures radicales et révolutionnaires, reconnaissant des titres sérieux aux noms qui ont été usités pendant de longues années, je pense, avec beaucoup de bons esprits, que sous prétexte de justice à rendre aux anciens, certains réformateurs se plaisent à substituer des appellations de leur façon à des noms connus, et loin de rendre service à la science entomologique ils ne font qu'ajouter des difficultés nouvelles à une science qui n'en a déjà que trop. Un exemple servira d'explication.

Le genre Lacon, bien connu en Europe par une de ses 
espèces, où elle est l'unique du genre, ne compte pas moins, à l'heure actuelle, de 231 espèces répandues dans le monde entier.

Ce nom générique de Lrtcon, vieux d'un demi-siècle, a été accepté par ceux qui ont adjoint successivement tous ces congénères' à l'espèce typique, l'E. murinus de Linnée.

Tout en reconnaissant que l'origine de ce nom, dû à Castelnau, est entachée de quelque irrégularité, il faut bien admettre que la recommaissance qui en a été faite depuis plus de cinquante ans dans nombre d'ouvriges et par des savants tels que Germar ('), Erichson, Lacordaire, Klug, Boheman, Von Harold, etc., lui a reconstitué un état-civil, une légitimité aussi acceptable que beaucouj d'autres, et en tous cas préférable à ce nom noureau d'Archontas, par exemple, que certains réformateurs voudraient lui imposer.

Cette recherche à outrance de la paternité des noms peut servir d'amusement à quelques bibliophiles et mettre en lumière l'érudition de ceux qui s'y livrent, mais ne sert nullement la science, bien au contraire.

Sous prétexte de justice à rendre à d'anciens descripteurs, oubliés non sans raison, ces érudits ne se font pas faute de commettre des erreurs et des injustices à l'égard d'auteurs autrement sérieux; en voici la preuve.

Les mêmes réformateurs dont je viens de parler voudraient substituer au nom de Corymbites, appliqué en 1834 par Latreille à quelques Élatérides, celui de Ctenicenus, domné par Stephens, en 1830, quatre ans aujaravant, aux mêmes insectes.

Au premier abord cela ne paraît que juste.

Mais si cette rectification était consommée, il surgirait

(1) Germar (Zeitsch. II, p. 260, 1810) a caractérisé avec détails le genre en question. Rćtablissons done ainsi la synonymie : Lacon (Casteln.) Germar Zcitschr., etc.

Pas n'est besoin d'un nom nouveau! 
des critiques qui ne manqueraient pas de faire remarquer que Latreille, ayant déjà fondé en 1817 un genre Ctenicère pour le Taupin double croix de Madagascar, Latreille est le légitime propriétaire du nom, et que Stephens, à qui on voudrait l'attribuer, l'avait lui-même indûment employé.

Laissons donc debout le nom de Corymbites.

J'ai cru devoir introduire quelques changements dans l'ordre de certaines tribus. L'une d'entre elles, celle des Mélanactites a été même supprimée et ses éléments dispersés dans d'autres, ainsi que j'en ai déjà donné les raisons antérieurement ('). On tronvera les genres qui composaient cette tribu répartis de la manière suivante: Les Alaolacon et les Aliteus dans les Asaphites, les Anaïssus et les Melantho dans les Crépidoménites, les Tibionema dans les Athoütes, les Ischius, près des Pyrophorus, les Menalactes et les Aphileus à la suite des Corymbites.

Le G. Beliophorus a été démembré ("); le G. Ictis supprimé, ce genre ayant été établi sur la femelle d'un Pleonomus.

Quelques modifications apportées à la composition des genres Ischiodontus, Eolus, Heteroderes, Corymbites, etc., seront justifiés en leurs lieu et place. Elles l'ont déjà été, du reste, dans des notes accompagnant des publications antérieures.

Le nom de Pristilophus a été rétabli comme nom générique. Les insectes qui le composent sont un démembrement des Corymbites ainsi qu'il a été expliqué dans une notice sur les Élatérides du Chota-Nagpore ( $\left.{ }^{(}\right)$.

J'ai dit tout à l'heure que j'avais apporté à la répartition géographique plus d'importance qu'autrefois.

Les Psephus, les Agonischius, les 1schiodontus, genres deve-

(1) Révision, p. 110.

() Ibid, , p. 205.

(3) Ann. Soc. ent. Belg., Bull, 1890 
nus tous trois très nombreux, sont reliés entre eux par des espèces en quelque sorte intermédiaires. Si l'on ajoute à leurs caractères que les premiers constituent la masse des Dicrépidiites de l'ancien continent, de l'A frique principalement, et que les deux autres appartiennent exclusivement à l'Amérique, leur classifiation, dans la tribu ci-dessus, en devient plus aisée et plus naturelle.

De même pour les Eolus, genre essentiellement américain et d'où j'ai retiré certaines petites espèces de l'Inde pour les réunir aux Heteroderes.

J'en dirai autant pour les Horistonotus d'Australie et de l'Inde que j'ai transportés dans les vrais Cardiophorus, toujours pour des considératious géographiques. Il est vrai que les caractères génériques proprement dits en reçoivent quelque atteinte, mais quel est le genre, riche en espèces, qui ne doive souffrir des exceptions pour pouvoir encadrer toutes les recrues qui ne cessent de lui arriver tous les jours.

La synonymie a été réduite au strict nécessaire, les noms tombés dans l'oubli et ceux qui ne se retrouvent que dans les catalogues étant exposés, d'autre part, dans les ouvrages descriptifs auxquels celui-ci renvoie.Il n'est fait d'exceptions que pour certains noms de Dejean et de Sturm, encore usités dans quelques collections.

Décembre 1890. 


\section{CATALOGUE MÉTHODIQUE}

\section{DES \\ É L A T É R I E S}

Tribu I.

AGR YPNites.

Front plat ou concave, généralement dépourvu de rebord transversal en avant, au moins sur la ligne médiane. Antennes courtes chez presque tous. - Prosternum ayant de chaque côté ses sutures ouvertes pour y recevoir et cacher les antennes au repos ('). Pubescence souvent rude ou squamiforme.

\section{Tribu II.}

\section{ALAïtes.}

Front concave, sans carène transversale en avant. Mandibules simples. - Antennes courtes, parfois flabellées chez les mâles et reçues à la base dans de courts canaux prothoraciques. - Mésosternum allongé, à bords saillants, séparé du métasternum par une suture distincte. - Tarses courts, revêtus en dessous de poils courts formant une brosse continue, ou des pelottes, jamais lamellés. - Corps souvent marbrés par des poils squamiformes. - Taille grande.

\section{Tribu III.}

Chalcolépiditites.

Tête concave, son bord antérieur variable. - Mentonnière grande. - Métasternum et mésasternum confondus ensemble,

(1) Les caractères principaux sont imprimés en italiques. 
re dernier faisant suite au premier sans suture distincte entre en..-- Tarses comprimés, munis de brosses ou de lamelles en dessous. - Corps glabre ou revêtu de poils squamiformes, de couleur brillante dans le premier cas, toujours vive et élégante. - Taille grande.

\section{Tribu IV.}

OXYNOPTÉRITES.

Tête concave, sans rebord en avant. - Mandibules simples, saillantes, brusquement recourbées. - Palpes longs. Antennesflabellées chez les mâles. - Epimères métathoraciques larges. - Tarses sans lamelles. Taille grande, parfois yigantesque pour la famille.

\section{Tribu V.}

TÉTRALOBITES.

Front très épaissi en avant, montrant une plaque uasale aussi haute que large, à peu d'exceptions près. - Antennes fortement flabellies chez les mâles. - Parapleures et épimères métathoraciques très larges. - Tarses quadrilamellés en dessous. - Taille très grande.

\section{Tribu VI.}

\section{DicréPIDHTES.}

Front fortement rebordé en avant. - Sutures prosternales concaves, généralement munies de canaux antennaires. Tarses comprimés, les deuxième et troisième articles au moins laminifìres, le quatrième petit, simple, jamais cordiforme. - Ongles simples. - Ecusson non cordiforme.

\section{Tribu VIr.}

\section{Eudactylites.}

Front caréné transversalement en avant. - Prosternum muni de sutures latérales droites, fines, rectilignes. - Tarses 
courts, très dilatés, munis de lamelles ou de brosses en dessous. - Ongles simples. - Ecusson non cordiforme.

\section{Tribu VIII.}

\section{MONOCRÉPIDITES.}

Tête légèrement convexe, rarement aplatie, le front rebordé en avant. - Sutures latérales du prosternum fines et rectilignes, à de rares exceptions près. - Hanches postérieures dilatées en dedans. - Tarses à article 4 lamellè ou dilaté, cordiforme. - Crochets simples.

\section{Tribu IX.}

\section{ELATÉRITES.}

Tête convexe à front rebordé en avant. - Prothorax plus étroit en avant qu'à la base. - Prosternum médiocrement large, ses sutures rectilignes ou concaves. - Hanches postérieures brusquement élargies en dedans. - Tarses simples, leurs crochets non pectinés. - Ecusson non cordiforme.

\section{Tribu $\mathbf{x}$.}

\section{Physorhinites.}

Tête convexe, à front rebordé, arrondi en avant.- Flancs prothoraciques toujours munis de canaux antennaires au sommet des sutures. - Hanches postérieures fortement dilatées en dedans. - Tarses courts, leurs deux premiers articles simples, le troisième laminifère, le quatrième très petit, confondu avec la base du cinquième. - Ongles simples. - Ecusson non cordiforme.

\section{Tribu XI.}

\section{Pomachilmtes.}

Tête très convexe, front rebordé. - Prothorax généralement allongé, plus large et plus épais en avant qu'en arrière. - Prosternum médiocrement large, ses sutures plus ou 
moins concaves et creusées en avant. - Tarses simples ou l'mn des articles élargi ou lamellé. - Corps étroit et allongé.

\section{Tribu XII.}

\section{CryptohypNites.}

Front grand, rebordé en avant. - Prosternum large, mresque toujours dilaté au milieu, en sorte que ses sutures sont convexes. - Hanches postérieures très larges en dedans, brusquement rétrécies en dehors. - Tarses ordinairement simples, leurs crochets jamais pectinés. - Taille très petite.

\section{Tribu XIIr.}

\section{CARdiophorites.}

Front arrondi et rebordé en avant. - Ecusson toujours cordiforme. - Prosternum assez large, ses sutures parallèles, sa saillie postérieure courte. - Bords de la fossette mésosternale verticaux. - Hanches postérieures fortement dilatées en dedans.

\section{Tribu XIV.}

\section{MÉlanotites.}

Front rebordé en avant. - Sutures prosternales concaves, canaliculées ou simples. - Hanches postérieures généralement étroites. - Ecusson non cordiforme. - Crochets des tarses toujours pectinés.

\section{Tribu XV.}

\section{Aтноїтеs.}

Front généralement subquadrangulaire, plat ou concave, caréné transversalement en avant. - Sutures prosternales rectilignes. - Hanches postérieures étroites. - Articles des tarses généralement simples, quelquefois les $2^{\mathrm{e}}$ et $3^{\mathrm{e}}$ épaissis. - Crochets des tarses simples. 


\section{Tribu XVI.}

PyROPHORITES.

Front sans carène antérieure, si ce n'est au-dessus de l'insertion des antennes, généralement concave, les crêtes sus-antennaires fortes, transversales dans leur portion interne. - Des vésicules phosphorescentes vers les angles postérieurs du prothorax, chez presque tous.

\section{Tribu XVII.}

Corymbitites.

Front dépourvu de carène transversale en avant, au moins sur la ligne médiane, de forme généralement carrée, aplati ou concave, incliné légèrement. - Prothorax dépourvu de vésicules phosphorescentes. - Hanches postérieures étroites, à lame extérieure complète et peu à peu atténuée en dehors.-Articles des tarses ni dilatés ni lamellés.

\section{Tribu XVIIr.}

CRÉPIDOMÉNITES.

Front sans carène transversale en avant, ordinairement acuminé, peu déclive, aplati ou concave. - Hanches postérieures peu à peu atténuées de dedans en dehors, à lame extérieure complète. - Articles 2-4 des tarses dilatés, triangulaires ou obcordiformes.

\section{Tribu XIX.}

Asaphites.

Front sans carène transversale en avant, généralement carré, peu déclive, plat ou concave. - Hanches postérieures étroites, peu à peu atténuées en dehors, à lames extérieures complètes. - Articles 2 et 3 des tarses lamellés ou sublamellés, le quatrième petit. 


\section{Tribu $\mathbf{X X}$}

Allotrites.

Front non caréné, plus ou moins penché, plat ou concave. - Hanches postérieures à lame extérieure complète. Articles 3 et 4 des tarses lamellés, au moins anx pattes antérieures.

\section{Tribu XXI}

\section{Dimites.}

Front non caréné en avant, peu déclive, aplati. - Hanches postérieures ì lumes extérieures incomplètes, c'est-à-dire oblitérées dans leur portion externe. Tarses simples ou lamellés.

\section{Tribu XXII.}

HypodÉstTES.

Front légèrement convexe, les fossettes antennaires grandes, les crêtes sus-antennaires obliques et courtes, ne cachant pas l'insertion des antennes. - Bouche inférieure. Sutures prosternales courbes. - Hanches postérieures étroites. - Tarses à articles 2-4 lamellés.

\section{Tribu XXIII.}

\section{Cardiorhinites.}

Front un peu convexe, les fossettes antennaires assez grandes et peu profondes, les crêtes sus-antennaires arquées, jeu saillantes. - Bouche inférieure. - Labre grand, sillonné et paraissant bilobé. - Suture posternale fine, légèrement courbe. - Mésostemum ordinairement vertical et saillant dans sa partie postérieure. - Tarses simples.

\section{Tribu XXIV.}

LUDHTES.

Front bombé, sans carène en avant, les crêtes sus-antennaires obliques, yeu scillantes. - Bouche inférieure. - Labre grand. 
- Mandibules perpendiculaires. - Sutures prosternales courbes. - Tarses simples ou lamellés. - Crochets non pectinés.

\section{Tribu XXV.}

Adrastites.

Front sans carêne transversale en avant, bombé, les crêtes antennaires obliques, la bouche inférieure. Labre fléchi en arrière. - Crochets des tarses pectinés.

\section{Tribu XXVI.}

\section{CAMPYlites.}

Front caréné ou non en avant. - Mandibules saillantes, droites à la base, brusquement courbées au bout. - Yeux souvent globuleux et saillants. - Prosternum dépourvu de mentonnière. - Hanches moyennes presque contiguës et rétrécissant fortement la fossette mésosternale en avant, et la saillie antérieure du métasternum en arrière. - Tarses pubescents en dessous.

\section{Tribu XXVII.}

Plastocérites.

Facies de Cébrionides. - Antennes généralement pectinées chez les mâles. - Elytres des femelles très raccourcies chez la plupart et laissant plusieurs segments abdominaux visibles en dessus. - Dernier article des palpes cylindriques. - Mandibules saillantes. 



\section{TRIBU I. AGRYPNITES.}

Prothorax muni, en dessous, de deux rainures formées par l'écartement des lèvres des sutures prosternales et destinées à loger et cacher les antennes au repos.

\section{Agrypnus.}

Esces. Thon Archiv. II, 1, p. 32.

\section{Amaurus CAst.}

Rainures prosternales entières et droites. - Carènes des angles postérieurs du prothorax toujours très longues et parallèles au bord même. - Taille généralement grande. - Couleur uniforme, allant du noir au ferrugineux, jamais variée. - Se rencontre dans les régions chaudes, principalement dans l'ancien continent.

Ier Groupe.

Europe, Afrique, Arabie, Syrie, Perse.

caliginosus CAND. Monogr. I, 26 . . Sénégal, Guinée. E. laticollis, Hope? Ann. nat. Hist., 1843. notodonta Latr., in Caillaud, Voy. à Méroé.

Ins . Sénégal, Soudan, Haute-Egypte, Abyssinie, Sicile. senegalensis CAST. Hist. nat. I, 137.

hymerensis RAGUSA, Natur. Sicil. 1881. substriatus CAND. Monogr. I, p. 25. Sénégal, Guinée, Congo. gutturosus Farrm. Ann. Soc. entom. Fr. 1887,

p. 148. . . . . . . . . . . Somalis. 
infuscatus Kuva, in Peters, Reise n. Mossam-

bique, 1855, p. 647 . . . . . . . Mozambique. puber Caxd. Monogr. I, p. 30. . . . Sénégal, Guinée.

tropicus Hope? Ann. nat. Hist., 1843.

attenuatus CANd. Révis. Mon. Élat., 1874, p. $4 . \quad$ Guinée. Bradshawi CAND. Élatér. nouv., fasc. IV, p. 2. Zumbèze. longicollis CAND. Ibid. . . . . . . . Abyssinie. parallelicollis CAND. Ibid. . . . . . . Kordofan. crassiusculus CAND. Monogr. I, p. 30 . . Sénégal. australis CANd. Révision, p. 5 . . . . . . L. N'Gami. pubescens CAND. Ibid. . . . . . . Sénégal.

Var. a. Bocandei CAND. Monogr.I, p. 32.

rufus CAND. Monogr. I, p. 33 ... . . Afrique équatoriale. pumilus CANd. Élatér. nouv., fasc. IV, p. 3 . . Abyssinie. Dewalquei CAND. Monogr. I, p. 33. . . . . Benguela. Peringueyi CAND. Élat. nouv., fasc. IV, p. 2 . Cap. Badeni CAND. loc. cit. . . . . . . . . . Isubu. Olcesi FAtrM. Journ. Le Naturaliste, 188t . . Mogador. arabicus CAND. Révis. Mon. Élat., p. 6 . . . Arabie. judaicus Reiche, Ann. Soc. entom. Fr., 1886,

p.418. . . . . . . . Syrie, Mésopotamie. persicus CANd. Révision, p. 6 . . . . . . Perse. maculicollis Gerst. B. z. Insekt. Zanzib, p.53. Zanzibur. luridus FABR. Entom. System., p. 11, 217, 4.

Arabie, Hindoustan occidental.

a Groupe.

Indes orientales, Chine, Japon, Archipel malais, Nlle Guinée, Polynésie.

fuscipes FABr. Syst. Eleut. II, p. 224. . . . Hindoustan. rufipes CAND.Révision, 1874, p. 7 . . . . Pondichéry. opacus CAND., loc. cit., p. 8. . . . . . Malacca. rubiginosus CANd., loc. cit., p. 9 . . . Borneo, Sumatra. æqualis CAND. Monogr. I, p. 25. . . . . . Java. sondaicus CAND. loc. cit., p. 33. . . . . Malaisie. 
punctatus CAND. loc. cit., p. 26. . Bengale, Annam, Sihlet, Birmanie. mostus Cand. loc. cit., p. 34. . Hindoustan mér. Ceylan. funestus $\mathrm{CAND}_{\mathrm{AND}}$ loc. cit., p. 35.

gilvus CAND. Élat. nouv. I, 1865.

attonitus CAND. Révision Mon. Élat., p. 10. Hindoustan. bipunctatus CAND. Monogr. I, p. 29 . . . Hindoust. sept. lacertosus CAND. loc. cit., p. 38, pl. I, fig. 1 . Sihlet. ocellatus CAND. loc. cit., p. 39 . . . . . . Ind. or. fusiformis CAND. loc. cit., p. 39. . . . . . Chine mér. Tippoi CaND. Élat. nouv. fasc. IV, p. 3 . . . Deccan. Renardi CAND. Bull. Soc. entom. Belg., 1890. Bengale. cinereus CAND. Monogx. I, p. 40 . . . . . Indes or. bifoveatus CAND. Tbid., p. 41. . . . . 1. Philippines. tomentosus FABR. Ent. syst. suppl., p. 138. . ” javanus CAND. Monogr. I, p. 44. . . . . . Malaisie. Hageni CAND. Notes Leyd. Mus., 1888 . . . Sumatra. sobrinus CAND. Tbid. . . . . . . . . . » mucronatus CAND. Monogr. I, p. 42 . . . . Borneo. politus CAND. Ibid., p. 43 . . . . . . Chine, Formose. resectus CAND. Ibid., p. 45 . . Moluques, Nouv. Guinée. soricinus CAND. Élat. nouv. fasc. III, p. 1 . Nouv. Guinée. Gestroi Cand. Ann. Mus. Gênes, 1880, p. 188 . pacificus CAND. Élat. nouv. III, p. 1 . . . Woodlark.

\section{3e Groupe.}

Australie.

Mastersi Mac-Leay, Trans. Soc. ent. N. S.

Wales II, p. 249 . . . . . . . . . Australie sept.

Var. latior. Ejusd., loc. cit.

\section{Groupe.}

Amérique.

Sallei Lec. Am. phil. Trans. X, p. 491. Éts-Unis du Sud. Schotti Lec. loc. cit., p. 492 . . . . . . . Rio Grande. 


\section{Scaphoderus.}

CAND. Monogr. I, p. 46.

Prothorax fortement excavé en dessus.

Riehli CAND. loc. cit., p. 47 . . . . . . Natal.

\section{Adelocera.}

(Latr.) Cand. Monogr. I, p. 46.

Sutures prosternales entières; antennes courtes, dentées à partir du 30 article; tarses sans lamelles; des sillons tarsaux plus ou moins accusés. - Cosmopolite.

\section{section I.}

Ėlstres ponctuées, les points ne formant pas neuf stries distinctes. carbonaria Schraxk, Énum., 1781 . . Europe centrale. atomaria FABr., CAND., Monogr.

punctata Herist. Beschäft.

pulverulenta Pavz. Entom. Germ.

sparsa CAND. Élatér. nouv. I, p. 6, 1865 . . . Californie. graeca CAND. Monogr. I, p. 61 . . . . . . Grèce, Syrie. drusa Mars. Abeille VI, p. 390.

lepidoptera Grluexh. Ins. suec. I, p. 379 . Europe septentrionale, Sibérie.

chrysoprasa Herbst, Käf. X.

Grafi Scharmot. Faunus I.

altaica CAND. Élat. nouv. fasc. III, p. 2 . . Altai. aurorata SAy, Trans. Am. Ph. VI, p. 181 . Neu Hampsh. impressicollis SAY, Ann. Lyc., I, p. 260 . . Etate-Unis. senitis Germ. Zeitsch. II.

rorulenta Lec. Proc. Acad. Ph. 1859, p. 283 . Etats-Unis. pyrsolepis LEc. loc. cit., 1866.

coca CAND. Révision, p. 18 . . . . . Guatemala. mixta CAND. Élat. nouv. $\Pi$, p. 5. . . . . . Mexique. 
massula CAND. Ibid. III . . . . . . . Mexique. funebris Solsk. Hor. Ent. Ross., 1882 . . . Sarapchan. tristis KraAtz, Deutsch. Zeitsch. XXVI. incompta KraAtz, Ibid., p. 319 . . . . . . . Samurcande. lithophila CAND. Monogr. I . . . . . . Egypte. conspersa GrLLEnH. Ins. suec. I, p. 377 . . Europe bor., Sibérie. brevicollis LEc. Trans. Am. Ph. X, p. 491 . . Lac Supér., S. Nevada. obtecta SAx, Ibid. VI, p. 181. . . . . Caroline du Nord. fasciata Lrnné, Syst. Nat. I, II, p. 655 : . . . Europe. incequalis De GeEr, Mém. toeniata Panz. Ent. Germ.

profusa CAND. Monogr. I, p. 54. . . . . Orégon. cavicollis Lec. Trans. Am. P. X. vetusta WALkER, Nat. Vancouv. II, 1866.

marmorata FABR. Syst. Éleuth. II, p. 227 . . États-Unis centr. et mérid. quercea Henbst, Fuëssl. Arch., V.1784, p.113. Europe centr. quercina LINNí, Syst. Nat. varia OL. Entom. II, p. 32. - CAND. Mon.

discoidea WEBER, Obs. entom., p. 77. - $\mathrm{S}_{A Y}$, Tr. Am. P. VI. . . . . . . États-Unis centr. et mér. cruenta OuIv. Entom. ornaticollis CAst. Hist. nat. pennata FABR. Syst. Elat. - CAND. Mon. chilensis Solmer, in Gay. Hist. de Chile V, p. 7. - CAND. Monogr. . . . . . lihili mér. Oliveri CAND. Révision, p. 20. . . . . . Darjeeling. unicolor CAND. Ibid., p. 21. . . . . . . Perse. avita SAY, Trans. Am. Phil. VI, p.182 . . Wisconsin. linearis CAND. Élat. nouv. I, p.6 . . . . Guyane. Mâklini CANd. Ibid. . . . . . . . Japon mér. insularis CAND. Élat. nouv. IV, p. 4 . . . .I. Andamans. 
tenebrioides Cand. Monogr. I, p.61 . . . Madagascar. aterrima CAND. Élat. nouv. IV, p. 4 . . . . ” madida CAND. Ibid. . . . . . . . . . . ” inflata CAND. Monogr. I, p.62 . . . . . » dorsalis CAND. Ibid. . . . . . . . . . ” pectoralis FAIRM. Stett. Zeit., 1883, p. 246 . ” maculata LEc. Proc. Acad. Phil., 1866, p. 389. Philadelphie. calabarica CAND. Révision, p.23 . . V. Calabar, Gabon. aberrans CAND. Ibid. . . . . . . . . Caracas. laticollis CAND. Monogr. I, p. 59. . . . . . Cayenne. angustata Philippi, Ann. Univ. Chil. V, p.743. Chili.

\section{Section II.}

Élytres nettement ponctuées-striées.

cribrata CAND. Monogr. I, p. 63 . . . . . Malacca. lacerta CAND. Élat. nouv. I, p. 7.

geographica Cand. liat. nouv. I, p. 7 - Borneo, Malacca. spurca CAND. Révision, p.25. . . . Laos, Cochinchine. collisa CAND. Élat. nouv. IV . . . . . . Silkim. aurulenta CAND. Élat. nouv. I, p. 6. . . . Ceylan. luzonica CAND. Élat. Philipp., 1875 . . . I. Philippines. Wallacei CAND. Révision, p. 26. . . . . Borneo. cincta CAND. Élat. nouv. II, p. 6. . . . . . Sumatra. tumulosa CAND. Élat. Birman., p. 2 . . . Tériassérim. massula CAND. Élat. nouv. III, p. 2 . . . Mexique. mexicana CAND. Monogr. I, p. 70 . . . . ” inæqualis CAND. Ibid, p.67 . . . . Nouv. Grenade. subcostata CAND. Ibid., p.69 . . . . Guadeloupe. pollinaria CAND. Ibid., p. 68. . . . . . . Brésil. setosa CANd. Révision, p. 29 . . . . . . . Para. adspersa CAND. Tbid., p.30 . . . . . Guatémala. modesta Borsd. Faun. Océan., p. 108 . Régions tropic. univ. pruinosa, squalida FAIRM. Rev. Zool., 1849. nigroplagiata BцANch. Voy. Pôle Sud IV,p. 85. 
pectinata CAND. Élat. nouv. I, p. 8. . . . . Guyane. Mekrani Cand. Ibid. IV, p. 5 . . . . . Béloutchistan. Ghabannei Guér. Icon. Ins. Règn. anim.,p.4. Para, Guyane. flammula Br. Voy. Pôle Sud.

palliata LATR. Voyag. Humboldt, I, p. 154. . Amér. centr., Nouv. Gren.

Chapuisi CAND. Monogr. I.

Jacquieri CAND. Monogr. I, p. 64 . . . . . Guyane.

mamillata CAND. Élat. nouv. I, p. 7 . . . . ”

rubra Perty, Delect. Anim., p. 20 . . . . . Brésil.

brasiliensis CASt. Silb. Rev. IV.

Castelnaui CAND. Élat. nouv. IV, p. 5 . . . Bahia.

\section{Dilobitarsus.}

Latr. Ann. Soc. ent. Frr. III, p. 142.

Caractères des Adelocera, avec les tarses lamellés.

petiginosus Germ. Zeitschr. II, p. 246 . . . Brésil. tessellatus CAND. Révision, p. 32 . . . . ”

minutus CAND. Élat. nouv. II, p. 6. . . . . Bahia. nebulosus CAND. Révision, p. 32 . . . . Guyane. abbreviatus CAND. Monogr. I, p. 78 . . . Brésil. cuneatus CAND. Élat. nouv. I, p. 8 . . . . Guyane. columbianus C $\mathrm{CND}_{\mathrm{N}}$. Monogr. I, p. 78. . . Nouv. Grenade. subsulcatus CAND. Révision, p. 34. . . . Equateur. Deyrollei CAND. Monogr. I, p.79 . . . . Nouv. Grenade. cariosus CAND. Élat. nouv. IV, p. 5 . . . . ” irroratus CAND. Monogr.I, p. 80 . . . . . ” lignarius CAND. Ibid. . . . . . Uruguay, Rép. Argent. quadrituberculatus CAND. Ibid., p. 81 . . . Brésil mérid. bicornis CAND. Tbid., p. 82. . . . . . . . Brésil. nubilus CAND. Ibid., p. 83 . . . . . . Nouv. Grenade. bidens FABR. System. Eleuth. II, p. 227 . . . Amér. trop. tuberculatus Latr. Ann. Fr., 1834. 
inopinus CANo. Révision, p. 35 . . . . . Nicaragua. Eloini CAND. Ibid. . . . . . . . . . Amér. centr. gracilis CAND. Ibid. . . . . . . . . Para. cornutus CAND. Elat. nouv. I, p. 8. . . Afrique équat. bacillus CAND. Ibid. II, p. 3. . . . . . Abyssinie. filiformis CAND. Ibid. . . . . . . . . . ” Raffrayi CAND. Ibid. II, p.6. . . . . . . Zanzibar.

\section{Anacantha.}

Sou. in GAY, Hist. Chile V, p. 18.

Comme les Dilobitarsus, mais à antennes plus longues et ne se cachant pas entièrement dans les cavités thoraciques.

sulcicollis Solier, in Gay, Hist. Chile V, p. 18. Chili. vitticollis FAIRM. Rev. Zool., 1860 p. 268 . . I. Chiloe. crux PHwLIP. Stettin. Zeit., 1860, p. 247. . . marmorata CAND. Révision, p. 38. . . . . Fairmairei CAND. Élat. nouv. III, p. 3 . . .

\section{Anaspasis.}

CANd. Élat. nouv. III, p. 4.

Front d'Eucnémides; hanches post. élargies extérieurement. fasciolata CAND., loc. cit.

Cliti.

\section{Acrocryptus.}

Cand. Révision, p. 39. - Cryptotarsus PHI.. ater PmL. Stett. Zeit., 1873, p. 308.

Chiti.

\section{Hexaulacus.}

Cand. Révision, p. 40.

Six sillons tarsaux.

Reedi CAND., loc. cit. 


\section{Ocneus.}

CAND. Monogr. I, p. 84.

Antennes munies de lamelles, prothorax tuberculeux.

limbatus CAND., loc. cit., p. 85 . . . . . . Brésil.

\section{Eimolus.}

CAND. Monogr. I, p. 178.

Antennes lamellées, prothorax non tuberculeux.

linearis CAND. Monogr. I, p.179. . . . . . Brésil mérid.

\section{Hemicheus.}

Cand. Monogr. I, p. 180.

Tarses lamellés, sillons antennaires incomplets.

caffer CAND. Monogr. I, p. 181 . . . . . . Cafrerie. adspersulus Kr. Peters Reis., p. 196 . . . . Mozambique.

\section{Optaleus.}

CAND. Monogr. I, p. 86.

Antennes pectinées; tarses lamellés.

limbatus CAND. Monogr. I, p. 87 . . . . . Brésil. cribratus BLANCH. Voy. de D'Orb. VI, ins.

$2^{\circ}$ part., p. 144 . . . . . . . . . . . ‘ ”

paleolatus CAND. Monogr. I, p. 88. . . . . ”

fasciatus CAND. Ibid., p. 89 . . . . . . . . ”

argentatus CAND. Révision, p. 42. . . . Amazone.

\section{LACON.}

(Casteln.) Germar, Lacordatre, Cand. Monogr.

Brachylacon Mots. - Archontas Goz.

Articles 2 et 3 des antennes petits ; sillons sternaux fermés en arrière de telle sorte que les antennes doivent se recourber pour pénétrer dans leur cavité; tarses sans lamelles; cosmopolite. 


\section{Groupe}

Africains.

turbidus Germ. Zeitsch. II, p. 263. Madagascar. antiquus CAND. Monogr. I, p. 100 nodifer Kutg. Ins. Madag., p. 62 eximius CAND. Monogr. I, p. 101 ornatellus CAND. Élat. nouv. IV, p. 6. . . " " gibberosus Cand. Ibid.

nodicollis Cand. Monogr. I, p. 104. sinuatus CAND. Ibid., p. 103 . grisescens CAND. Révision, p. 52 . crenatus KuUG. Ins. Madagasc., p. 66

terrenus GERM. Zeitschro, p. 262.

pictus CANd. Élat. nouv. II, p. 7. decoratus CAND. Ibid. III, p. 6. Badeni CAND. Ibid. II, p. 7 porosus KuvG. Ins. Madag., p. 65 Goudoti Cand. Monogr. I, p. 106 leprosus CAND. Tbid., p. 107. vestitus KLug. Ins. Mad., p. 64 . tumidicollis Cand. Monogr. I, p. 108. irroratus KutG., loc. cit, p. 65 confusus Cand. Monogr. I, p. 109. Jansoni FATRM. Ann. Fr., 1871, p. 40 maculosus CAND. Révision, p. 53 Fairmairei Caxd. Elat. nouv. IV, p. 6 Pipitzi CAND. Ibid. tripartitus CAND. Révision, p. 54 albopictus CAND. Monogr. I, p. 104 subocellatus CAND. Elat. nouv. III, p. 6 . mysticus Cand. Monogr. I, p. $10 t$. sordidus CAND. Tbid., p. 114 . Sénégal. palliditarsus CAND. Ibid., p. 120 . . . . . Cap. 
albitactus CANd. Révision, p. 55 . . . . . V. Calabar. asper CAND. Ibid. . . . . . . . . . Guinée.

fictus CAND. Élat. nouv. II, p. 7 . . . . . . Madagascar. minor CAND. Monogr. I, p. 126 . . . . . " "

nanus Bон. Ins. Caffr. I, II, p. 420 . . . . . Cafrerie. troglodytes Вон., loc. cit., p. 419.

tumidipennis CAND. Élat. nouv. II, p. 7. . . Zanzibar. occidentalis CAND. Monogr. I, p.130. . . . Sénégal. signatus CAND. Élat. nouv. III, p. 6 . . . . Zanzibar. ethiopicus CAND. Ibid., p. 7 . . . . . . . ” foedus Cand. Monogr. I, p. 123 . Sénégal. Guinée, Abyssinie. muscerda CAìd. Révision, p. 57 . . . . Cap. granulosus Esch.?

caffer CAND. Élat. nouv. III, p. 7 . . . . . Cafrerie. decipiens BoH. Ins. Caffr. I, II, p. 417. . . . ” serricornis Quedenf. Bull. Berl., 1886 . . . Congo. pictiventris CAND. Monogr. I, p. 127 . . . . Madagascar. latiusculus CAND. Flat. nouv. II, p. 7. . . . ” soricinus CAND. Monogr. I, p. 133 . . . . . ” latus CAND. Ibid., p.136. . . . . . . . . ” hydropicus FaIRMr. Natural., 1880 . . . . . I. Réunion. flavipes CAND. Monogr. I, p. 137 . . . . . ”

Maillardi Deyr. Maill. Réun. II, p. 4. nigrita Cand. Monogr. I, p. 137 . . . . . . Cap. amplicollis BоH. Ins. Caffr. I, II, p. 414. . . Cafrerie. hottentota CAND. Monogr. I, p.138 . . . . Cap. porcinus CAND. Ibid., p. 142 . . . . . . . Madagascar. interpunctatus KuUG. Ins. Madag. I, p.66 . ” scaber CAND. Monogr. I, p. 153 . . . . . ” bidivisus Cand. Révision, p. 58. . . . . . V. Calabar. Desjardinsi Cand. Monogr. I, p. 143. . . Ile Maurice. pænulatus Вон. Ins. Caffr. I, II, p. 415 . . . Cafrerie. parcus Вон. Ibid., p. 416 . . . . . . . . ” 


\section{Groupe II.}

Européens, Asiatiques ( 8 ).

A. Elytres marquées de stries ou de séries de points; côtés du métathorax munis d'un sillon oblique bien délimité, pour recevoir les tarses médians au repos.

parallelicollis CAND. Monogr., p. 118 truncatus Herbst, Käf. X, p.98 . . . Hindoustan sept.

cylindricus CAND. Monogr. I, p. 121.

molestus Cand. Monogr. I, p. 120 . . . . . ”

elliensis CAND. Révision, p. 59 . . . . . . Ceylan.

variatus CAND. Bull. Soc. ent. Belg., 1890 . B Bengale. angulosus CAND. Monogr. I, p.117 . . . Indes orient. inductus CAxd. Ann. Mus. Gênes, 1888 . . . Birmanie. taciturnus CAND. Révision, p. 60 . . . . L Laos. falsarius CAND. Ibid. . . . . . . . . Ceylan. colonicus CAND. Elat. nouv. III, p. 8. . . . Cochinchine. spretus CAND. Ibid. . . . . . . . . . . Borneo. transversus CAND. Monogr. I, p.123. . . . Birmanie. hispidulus CAND. Tbid., p. 126 . . . . . Malaisie. lutosus CAND. Ibid., p. 119. . . . . . . . Népaul. setulosus CAND. Élat. nouv. III, p. 8. . . Flores. defectus CAxd. Ann. Mus. Gênes, 1888, p. 668. Birmanie. setiger Bates, Proceed. zoolog. Society, 1866,

p. 348. . . . . . . . . . . Formose. pallidus CANd. Notes Leyd. Mus. IX, p.190 . Sumatra. molitor CAND. El. Philip. in Ann. Soc. ent.

Belg., 1875 . . . . . . . . . 1. Philippines. judex CANd. Révision, p. 62 . . . . . . Shang-Haï. multiforis CAND. Monogr. I, p. 117. . . . . Syhtet. fibrinus CAND. Élat. nouv. I, p. 9 . . . . Malaisie. reductus CAND. Élat. nouv. II, p. 7 . . . . Sumatra.

() Les espèces de la Nouvelle Guinée et de la Polynésie sont comprises dans ce groupe. 
modestus CaND. Monogr. I, p. 118. . . . Indes or. leucaspis CAND. Révision, p.63. . . . . . Malacca. haedulus CAND. Monogr. I, p. 122 . . . Hindoustan sept. fex Cand. Révision, p. 64 . . . . . . . . Malacca. consors CAND. Ibid., p. 64. . . . . . . Madras. mixtus CAND. Él. Birm. in Ann. Mus. Gên.,1888. Birmanie. comptus CAND. Révision, p.65 . . . . . . Siam. lustratus Cand. Bull, Soc. entom. Belg., 1890. Bengale. torrefactus CAND. Monogr. I, p. 128 . . . . Birmanie. jurulosus CAND. Él. Birm., Ann. M. Gên., 1888. coctus CANd. Révision, p. 66 . . . . . . . ” piger CAND. Élat. nouv. IV, p. 7. . . . . . Bengale. flavescens Cand. Révision, p. 66 . . : . . Ceylan. tostus Cand. Monogr. I, p. 120. Malaisie, Birmanie, Bengale. lupinosus CAND. Ibid., p. 130. . Birmanie, Siam, Malaisie. acervatus CAND. Él. Birm., in Ann. Mus.

Gênes, 1888

Birmanie.

tactus CAND. Révision, p. 67 . . . . . . . Siam. inops CAND. Ibid . . . . . . . . . . . Malaisie. melancholicus CAND. Bull.Soc. ent. Belg., 1890. Bengale. muticus Herbst, Käf. X, p. 93 . . . . Indes orientales. acerbus CAND. Élat. Birm. in Ann. Gênes,1888. Birmanie. Delesserti CAND. Élat. nouv. III, p. 9. . . . Neelgherries. pauper CAND. Ann. Mus. Gênes, 1878. . . . Java. afflictus CAND. Révision, p. 68 . . . . . Malacca. ovalis GERM. Ins. Sp. nov., p. 49. . . . . . Perse. minusculus CAND. Révision, p. 68. . . . . Bombay.

B. Métathorax dépourvu de sillons.

ornatus CAND. Monogr. I, p. 102 . . . . . Java. costicollis CAND. Ibid., p.116. . . . . Hindoustan sept. desquamatus CAND. Ibid. )) mustelinus Germ. Zeitsch. II, p. 264 . . . . Siam. fuliginosus CAND. Élat. nouv. I, p. 3 . . . Japon. 
Beccarii CAND. Ann. Mus. Gênes, 1878

Sumatra.

bipapulatus CAND. Élat. nouv. I, 1865

cinine.

Agr. orientalis Hope? Trans. London, 1845.

coarctatus CAND. Révision, p. 70 .

Himalaya:

formosanus Bates. Proc. Zool. Soc., 1866, II,

p. 348.

Formose.

birmanicus CAND. Révision, p. 71.

Birmanie.

pardalinus CAND. Élat. nouv. III, p. 9 . . . Himalaya.

nepalensis Cand. Révision, p. 72 . . . . Népaul.

scutellatus CAND. Monogr. I, III . . . . . Malaisie.

murinus Linse, System. Naturæ II, p. 655. . Europe.

nebulosus Razum. Hist. nat.

thoracicus Scoporr. Kokeili Kust.

binodulus Мотsсн. Etud. entom., 1860, p. 8 .

Japon. cordicollis CAND. Élat. nouv. I, p. 9 . . . . dorcinus Cand. Ann. Soc. ent. Belg., XVW,

p. 69 . . . . . . . . . 1. Philippines. cinerascens Cand. Ann. Gênes, 1878, p. 103 Nouv. Guinée. limosus CAND. Élat. nouv. III, p.10 . . . . »

Schwaneri CANd. Révision, p. 73. . . . Borneo. litigiosus CAND. Ibid. . . . . . . . . Malacca. insularis CAND. Ib., p. 74 . . . Maluisie, Nouv. Guinée. erinaceus CAND. Ibid. . . . . . . . . . Borneo. coenosus CAxd. Monogr. I, p. 114 . . . Hindoustan sept. sericans CAND. Ibid., p. 112 . . . . . . . Ceylan. furunculosus CAND. Mbid., p. 134 . . . . . Bengale. binus CAND. Élat. nouv. IV, p. 7. . . . . Simla. Beccarii Cand. Ann. Mus. Gênes, 1880, p. 192. Sumatra. consobrinus CAND. Monogr. I, p. 125. . Hindoustan sept. ellipticus CAND. Ibid., p.135. . . . . . Himaleya. propinquns $\mathrm{C}_{\mathrm{AND}}$. Tbid.

brunnipennis CAND. Ibid, p. 133 . . . . . Himalaya. argillaceus Solsky, Hor. S. Ross., 1871. Sibérie or., Corée. cimamomeus CAND. Révision, p. 76. 
Davidis Farrm. Ann. Soc. ent. Fr., 1878, p.109. Chine centr. prætermissus CAND. Révision, p. 76. . . . Indes orient. musculuș CAND. Monogr. I, p.141 . . . . . Chine mérid. tristis Bu. Voy. au Pôle Sud, p. 86. . . . Nouv. Guinée. brachychætus Kollar. Hügel Kashm. IV,

p. 506. . . . . . . . . . . . . Cachemire. pygmeus BAUdr. Abeille, 1871 . . . . . . Chypre. impressus CAnd. Ann. Mus. Gênes, 1878 . Nouv. Guinée. scrofa CAND. Ins. Lewis in Mém. Liége, $2^{\prime \prime}$ sér.

V, p. 4 . . . . . . . . . . . Japon.

Henzi König, Hor. Soc. Ross., 1888, p. 553. . Corée.

depressus CAND. Révision, p. 77 . . . . . ”

apodixus CAND. Élat. nouv. I, p. 9. . . . . Luçon.

cervinus Er. Beitr. z. zool. Meyens Reise in

Act. Leop. Carol., 1834.

macroderus CANd. Élat. nouv. I. Moluques, Nouv. Guinée. Wallacei CANd. Révision, p.80. . . . . Célèbes. glirinus CAND. Élat. nouv. I, p.11 . . . . . I. Fidjee. stricticollis FaIRM. Ann. Fr., 1881. . . . . 》 serricollis CAND. Monogr. I, p. 140 . . . . Java. serrula CAND. Ibid., p. 122 . . . . . . . Népaul. lapideus CAND. Ibid., p. 141 . . . . . . . Indes orient. asperulatus Cand. Ann. Mus. Gênes, 1878. Nouv. Guinée. crenicollis Ménétr. Cat. rais., p. 156. Cancase, Himalaya. sinensis CANd. Monogr. I, p. 139. Chine mér., Siam, Sumatra. spurcus CAND. Élat. nouv. I, p. 11 . . . . 1. Philippines. gracilis CANd. Révision, p. 83 • . Moluques, Nouv. Guinée.

C. Élytres à ponctuation diffuse.

obesus CAND. Monogr. I, p. 132 .

Ceylan. nebulosus CAND. Tbid., p. 115. ) succinctus CAND. Bull. Soc, ent. Belg., 1890 . Bengale. adspersus CAND. Monogr., p. 125 . . . Hindoustan sept. demissus CAND. Elat. nouv. IV, p. 8 . . . Bengale. 
tumens Cand. Ins. Lewis in Mém. Liège V,

$2^{\circ}$ sér., p. 4. . . . . . . . Japon. turgescens CAxd. Révision, p. $8 t$. . . Poulo-Pinany. sparsus CAND. Tbid., p. 85. . . . . . J Java. trifasciatus CAND. Ėlat. nouv., p. 10. . . . Japon mér., Ceylan, Bomeo, etc.

Brachylacon microcephahus Motsch. Et. ent. laxus CANd. Révision, p. 85 . . . . . . . Mysole.

\section{Groupe II.}

Australiens ( $\left.{ }^{(}\right)$.

caliginosus Gú̇rn. Voy. Coquil. Ins., p. 68. Australie mér. labiosus Cand. Révision, p. 86 . . . . . Australie occ. ferrugineus CAND. Ibid., p. 87. Aust. Nord-occ, Nickol-Buy. socius CAND. Tbid. . . . . . . . . . Cap York. crassus CAND. Ibid., p. 88 . . . . . . . . ”

assus CAND. Monogr. I, p.145 . . . . . . Austr. orient. laticollis CAND. Ibid., p.146 . . . . . . » ursulus CAND. Tbid., p. 147 . . Australie orient. et mérid. princeps CAND. Révision, p. 89 . . . . Cap York. compactus CAND. Élat. nouv. III, p. 10 . . Australie sept. gibbus CAND. Ibid.

rubiginosus CAND. Ibid. p. 11 . . . . . ”

Mac Leayi CAND. Ibid. . . . . . . . Port Denison. geminatus CAND. Monogr. I, p. 149 . . . Q Queensland. mamillatus Caxd. Ibid., p. 144 . . . Nouv. Galles du Sud. Deboulayi CAND. Révision, p. 89 . . . . . Nickol-Bay. humilis Erichs. Wiegm. Arch. 1842, I, p. $136 . \quad$ Tasmanie. parallelus CAND. Révision, p. 90 . . . . Queensland. variabilis CAnd. Monogr. I, p. 148. Nouv. Hollande, N. Zél. alternans MaC LEAy.

(1) Continent austral, Tasmanie et Nouv. Zélande. 
monachus CAND. Élat. nouv. III, p.12 . . Victoria. Gastelnaui CAND. Tbid. . . . . . . . Austr. occ. corvinus CAND. Ibid . . . . . . . . . . " marginatus CAND. Révision, p. 91. . . . Clarence Riv. palpalis CAND. Élat. nouv. III, p. 13 . . . . Cleveland B. fatuus CANd. Révision, p. 91 . . . . . . . Austr. occ. variolus CAND. Tbid., p.92. . . . . . . Queensland. dealbatus CAND. Élat. nouv. III, p. 13 . . . Cap York. pleureticus CAND. Révision, p. 93 . . . . Queensland. pictipennis CAND. Monogr. I, p. 150 . . . . Austr. mérid. porriginosus CAND. Révision, p. 93 . . . Austr. occid. guttatus CAND. Monogr. I, p. 151 . . . . . Victoria. marmoratus CAND. Révision, p. 94 . . . Queensland. plagiatus CAND. Élat. nouv. II, p. 8 . . . Port Denison. octavus CAND. Révision, p. 95 . . . . . . Queensland. Van de Polli Cand. Notes Leyd. Mus., 1888 Carpentaria B. costipennis GERm. Linn. entom. III, p. 181. . Adélaïde. carinulatus CAND. Monogr. I, p. 152 . . . Nouv. Galles S. sculptus CAND. Révision, p. 95 . . . . . . Austr. mérid. lacrymosus CAND. Ibid., p. 96 . . . . . . ” divaricatus CAND. Élat. nouv. I, p. 12 . . . ” Victoriæ CAND. Ibid., p. 12 . . . . . . Victoria. insignitus CAND. Révision, p. 98 . . . Queensland. cordipennis CAND. Ibid., p. 99 . . . . . . Albany. granulatus MAC LeAy. Trans. N. S.W. . . Austr. orient. punctipennis CAND. Révision, p. 99 . . . . Swan-Riv. gayndahensis Mac LeAY, Trans. N. S. W . . Austr. occid. maculatus MAC LeAY, Ibid. . . . . . . . ”

\section{Groupe Iv.}

Américains.

rectangularis SAY. Ann. Lyc. I, p. 263 . . États-Unis. mucoreus Lec. Am. Phil. Soc.Trans. X, p. 491 Georgie. curtus Lec. Ibid . . . . États-Unis du Sud et Mexique. 
brevis CAND. Monogr. I, p.156. . . . . Yucatan. scarrosus CAND. Ibid . . . . . . Guatemala. calamitosus CAND. Révision, p. 100 . . . . Oajaca. tuspanensis CAND. Monogr. I, p. 157. . . . Mexique. Lezeleuci CAND. Ibid., p. 158. . . . . . . » suillus CAND. Ibid., p. 159 . . . . . . . . ” Truquii CAND. Révision, p. 101 . . . . . ” dubius CANd. Monogr. I, p.160 . . . Now. Grenade. cribrosus Eschs. (Arrymuse) Thon Arch.1828. I. Sanduich.

\section{Meristhus.}

Cand. Monogr. I, p. 162.

Trachylacon pars Mots.

Sutures prosternales des Lacon; une forte saillie longitudinale au milieu de l'écusson.

lepidotus Paurs. de Beauv. Ins. II. . . . . Guinée. quadripunctatus CAND. MIonogr. I, p. 163. Sillet, Annam, Birmanie.

insignitus CAND. Révision, p. 103 . . . . Ceylan. pistrinarius CAND. Monogr. I, p. 164. . . . Bimanie. Perraudieri Fleut. Ann. Fr., 1889, p. 139. .A Annam. apicalis CANd. Révision, p. 103 . . . . Célèbes. minusculus CAND. Ibid. . . . . . . B Borneo. scobinula CAND. Monogr. I, p. 16t. Régions intertiopicules. texanus Horx. Tr. Am. Soc. 1871. setarius Chevr. Ann. Fr. 1867.

cristatus Horx. Trans. Am. p. Soc.1871, ]) 299.

Texus.

AGræUS.

Cand. Monogr. I, p. 164.

Trachylacon Mотs.

Caractères des Lacon. Prothorax unituberculeux, portant des saillies souvent pénicillées. 
Mannerheimi CAND. Monogr. I, p. 166 . . Java. Ritsemæ CAND. Notes Leyd. Mus., 1883. . . Sumatra. constrictus Rirs., loc. cit. III, p. 29 . . . . Java. cuniculus CAND. Révision, p.105 . . . . . Singapore. feroculus CAND. Tbid. . . . . . . . Sumatra. Mouhoti CAND. Ibid., p. 106 . . . Birmanie, Cambodge.

Pericus.

Cand. Monogr. I, p. 167.

Caractères des Lacon. Côtés du prothorax translucides.

nitidus Cand. Monogr. I, p. 167. . . . Hindoustan sept. rubicundus CAND. Révision, p. 107 . . . . m?

MyrModes.

CAND. Monogr. I, p. 168.

Ne diffère des Lacon que par la structure étranglée de la base du prothorax et des élytres.

akidiformis CAND. Monogr. I, p.169 . . . Raffle-Bay.

Triotarsus.

Germ. Zeitschr. II, p. 247.

Cavités sternales des Lacon; tarses lamellés.

cinctipes Germ. Zeitschr. II, p. 248 . . . . Madagascar. cuspidatus CaND. Monogr. I.

major CAND. Monogr. I, p. 172 . . . . . . »

mucoreus CAND. Ibid., p. 173. . . . . . . ”

albisparsus CAND. Ibid., p. 174. . . . . . ”

rugatus CAND. Ibid., p. 175 . . . . . . ”

suboculatus CAND. Élat. nouv. III, p. 13. . . ' ग

depressus CAND. Tbid., p. 14 . . . . . . . ”

nubilus Cand. Monogr. I, p. 175. . . . . Guinée.

fulvisparsus CAND. Révision. 
reductus CAND. Élat. nouv. III, p. 14 . . . Gabon. marmoratus CAND., loc. cit. II, p. 8 . . Zanzibar. armatus CAND., loc. cit. $\Gamma$, p. 8. . . . . . Madagascar. spinifer CAND. Ibid., p. 8 . . . . . . . . ” sulcatus CAND. Monogr. I, p. 176 . . . . . Guinée. fuscus Quedenf. Deutsch. entom. Zeitschr. 1886 Congo. squalescens Fatrm. Amn. Soc. Fr. 1871, p. 39. Madagascar. cuspidatus Krug. Ins. Madag., p. 66. . . . ” Boieldieui CAND. Monogr. I, p. 176. hastatus CAND. Monogr. I, p.175 . . . . ” aculeatus CAND. Ibid., p. 178. . . . . . . ” tuberculatus CAND. Révision, p. 109. . . Benguela. simplex CAND. Ibid., p. 110 . . . . . . Gabon. subserratus Quedenf. Deutsch. entom. Zeitschrift 1886.

Congo.

\title{
TRIBU II.
}

\begin{abstract}
ALAÏTES.
Téte penchée, concave en avant, les antennes, courtes, parfois flabellées chez les mâles, leur base reçue au repos dans des canaux prothoraciques; mandibules simples sauf de rares exceptions; tarses revêtus de brosses ou de pelottes en dessous; mésosternum séparé du métasternum par une suture distincte; corps généralement grand, revêtu chez presque tous de poils squamiformes de couleur variée, exceptionnellement glabre.
\end{abstract}

\section{Euphemus.}

Casteun. Silberm. Revue ent. IV, p. 7.

Antennes des mâles biflabellées; canaux prothoraciques très grands. 
quadrimaculatus FABR. Entom. syst. II,

p. 218. . . . . . . . . . Guinée, Sénégal. fasciatus DruRy. Ill. exot. Ins.

funerarius Bertol. Mem. Act. Bologn., série

II, IV, p.5 . . . . . . . . . . Congo, Mozambique.

EUMCEUS.

Cand. Révision des Élat. 1874.

Antennes biflabellées chez les mâles; pas de canaux antennaires.

Murrayi CAND. Révision, p. 113 . . . . . Madras.

Alaolacon.

CAND. Élat. nouveaux I, 1865.

Antennes remarquablement courtes, le $3 e$ art. grand, triangulaire, le dernier ovale ; sutures prosternales déprimées, non excavées.

cyanipennis CAND. Élat. nouv. I, p. 13 . . . Malacca. griseus CAND. Révision, p. 114 . . . . . Bankgok.

\section{Ctenicera.}

LATr. Règne anim. 1817.

Antennes des mâles de 12 articles, lamellées; des canaux prothoraciques.

nobilis Iutig. Wiedem. Arch., p. 1, 2, 116 . . Madagascar. bicruciata LAtr. Règne anim.

insignis KLUG. Ins. Madag., p. 66 . . . . . » lugubris CAND. Monogr. I, p. 206 . . . . . Mozambique. controversa KARSH, Sitz B. nat. Freund.1881,

p. 58

Guinée.

\section{LYCOREUS.}

Antennes de onze articles dans les deux sexes, celles des mâles flabellées.

Iphis Cast., Gem. et Har. Cat.

madagascariensis Gony, Ann. Fr. I, p. 385. Madagascar.

bioscellatus Sturi, Cat.

dux CAND. Monogr. I, p. 208 . . . . . . ”

regalis CAND. Tbid., p. 209. . . . . . . . » 
Goudoti Cast. Silb. Rev. IV, p. 9 . . . . Madagascar. triocellatus Cast. Ibid., p. 8. . . . . . ” cyclops Cand. Révision, p.116 . . . . . ”

Ebenani Heyden, Senck. nat. Ges. Francf. 1877. corpulentus CAND. Élat. nouv. IV, p.11. . . ” figuratus Har. (') Munch. Ent.Ver.1878, p.105. Congo. bicarinatus Quedenf. Deutsch. entom. Zeitschrift 1886.

\section{Aliteos.}

\section{Cand. Monogr. I, p. 197.}

Alaus africains à mandibules tronquées-bidentées.

Reichei CAND. Monogr. I, p. 197. . . . . .

\section{ANTHRACALAUS.}

Fatrm. Ann. Soc. entom. France, 1888, p. 349. Alaus CAND. Monogr. I, p. 216.

Alaus à téguments tout à fait glabres.

Westermanni CAND, loc. cit. . . . . Java. Moricei FaIRM., loc. cit. . . . . . . . . Cochinchine.

Alaus.

Eschs. Thon. Arch. II.

Calaïs CAND. Monogr.

Antennes courtes; mandibules simples; méso- et métasternum séparés par une suture transversale; corps écailleux et tachetés; taille généralement grande.

\section{Ier Groupe.}

Africains.

excavatus FABR. Syst. Eleuth. II, p. 230 . Sénégal, senegalensis Cast., Cand. Monogr.

(I) Je pense qu'il y a identité entre cette espèce et l'Alaus Hacquardi, ou grande aftinité. Dans le premier cas l'espèce étant un Alaus, et le nom de figuratus étant déjà appliqué dans ce dernier genre, le nom de Hacquardi, quoique postérieur, devrait ètre maintenu. Je ne connais pas le suivant. 
truncatipennis Вон. Ins. Caffr. I, II, p. 391 .

Guinée, Zanguebar, Cafrerie. elegantulus CAND. Monogr. I, p. 220.

Dohrni CAND. Élat. nouv. III, p. 15 . . . . Liberia. macer CAND, Élat. nouv. II, p. 8. . . . . . Gabon. Hacquardi CAND. Élat. nouv. III, 1882, p. 15 . Zanguebar. rudis CAND. Monogr. I, p. 221. . . . . . . Cafrerie. moerens Germ. Zeitschr. II, p. 254. . . Cafrerie inf., Cap. Revoilii FAIRM. Ann. Soc. ent. Fr., 1887, p.148. Usagura. cerberus CAND. Révision, p.123 . . . . . Dahomey. Macari CAND. Élat. nouv. IV, p. 10. . . . Louloua. nigrosignatus Qued. D. Entom. Zeitsch., 1886 Congo. Groquisii CAND. Élat. nouv. III, p. 15. . . . Gr. Bassam. tortrix CAND. Révision, p. 123 . . . . . . Mozambique. crucifer CAND. Élat. nouv. IV, p.9. . . . . Angola. Antinori CAND. Ibid., p.9 . . . . . . . . Abyssinie. sinuosicollis CASt. Silb. Rev. IV, p. 9 . . Sénégambie. detritus CAND. Monogr. I, p. 224 ... . . Cafrerie. Mniszechi CaNd. Ibid. . . . . . . . Gabon. hieroglyphicus Cand. Tbid., p. 225 . . . . Sénégal. marmoratus CAND. Ibid., p. 226 . . . . . _ 》 caffer Bон. Ins. Caffr. II, p. 390. . . . . . Cafrerie. atropos Gerst. Beitr. z. Ins. Zanz., III, 1871. Zanzibar. Gandezei Murr. Ann. nat. Histor., 1870. . Vieux-Calabar. stellio CAND. Élat. nouv. IV, p. 10 . . . . Isubu. tristis CAND. Ibid., p. 10 . . . . . . . . Congo. polyzonus Gerst. J. Hamb. Anst. I, p. 52 . . Massä̈.

\section{ge Groupe.}

Indiens.

(Europe, Asie, Nlle Guinée, Polynésie.)

A. - Écusson globuleux, échancré en avant.

speciosus Livne, Syst. Nat. II, p. 652. Ceylan, Travancore. 
podargus Caxd. Révision, p. 125 . . 1.. Philippines, Bojol. Senecterei CAND. Monogr. I, p. 207 . . . . Dekkan. merens Westw. Cab. or. Entom.

superbus Cand. Ann. Soc. ent. Belg., Bull. XVII, 1875 . . . . . . . . . . Mindanao. lacteus FABR. Syst. Eleuth. II, p. 230 . . . Malaisie, surtout Borneo.

Var. lymphaticus Thoмs. Rev. Zool. 1856.

Laportei CAND. Élat. nouv. III, p. 17 . . . Borneo N. O. Wallandi Cand. Notes Leyd. Mus. V, p. 207 . Sumatramér. pantherinus CAND. Élat. nouv. III, p. 16 . Mindanao. lophura Cand. Élat. nouv. I, p. 15 . . Borneo, Malacca. sculptus Westw. Cab. or. Entom., p. 72 . Hindoustan sept.,

Sikkim.

Vollenhoveni CANd. Élat. nouv. I, p. 14. Célèbes, I. Sanghir. Engelhardi Cand. Notes Leyd. Mus. V, p. 11 . 1. Saleyer. appendiculatus Herbst, Käf. X, p.330. . . Amboine. farinosus OL. Journ. Hist. nat.

\section{B. - Écusson plus long que large, déclive.}

Parreyssi Stev. Bull. mosc. I, p. 154 . Crimée, Asie min. Goryi Gonx. Mag. zool. 1832.

Cardoni Cand. Bull. Soc. entom. Belg., 1890 . Bengale. berus CAND. Élat. nouv. I, p. 15 . . . . Japon, Chine mér. nubilus CAND. Monogr. I, p. 230. . . . . Java. mortuus Thoms. Rev. Zool., 1856, p.473. . . Borneo. torquilla CAND. Monogr. I, p. 239.

sordidus Westw. Cab. of or. Entom., p. 72 . Hindoustan, Ceylan, Assam, Laos. lactellus CAND. Révision, p. 130. . . . . . Borneo. flguratus $\mathrm{C}_{A N D}$. Ibid. . . . . . . . . Malaisie. maculosus CAND. Ibid., p.13. . . . . Borneo N. O. cenchris CAND. Monogr. I, p. 23. . . B Birmanie, Laos. elaps CAND. Révision, p.132 . . Malaisie, Ceylan, Laos. Colffsi CAND. Élat. nouv. II, p. 17 . . . . Sumbawa. 
anguis CAND. Élat. nouv. I, p. 15

Laos.

Boreli CANd. Monogr. I, p. 229 . . . Ternate, Batchian. cerastes CAND. Élat. nouv. I, p. 16 . Nouv. Guinée occ., Moluques. velutinus CAND. Ann. Mus. Gênes XII, p. 189. Andä̈, Nouv. Guinée occ. acontias CAND. Élat. nouv. III, p. 18 . . N. Guinée orient., Fry River.

musivatus CAND. Élat. nouv. I, p. 16. . . . Java. regalis CAND. Ibid. . . . . Céram, Amboine, Boeroe. Platteuwi Cand. Ann. Soc. Belg. 1890, Bull.,

p.76. . . . . . . . . . . . Banjermassing. caprimulgus Cand. Révision, p. 136 .Birmanie, Bornéo occ. hurria CAND. Élat. nouv. III, p 17. . . . . Sumatra occ. Ritzemæ CAND. Not. Leyd. Mus. XI, 1889, p. 95. scops CANd. Révision, p. 137 . . . . . . . N. Guinée. Albertisi Cand. Ann. Mus. Gênes XII, p. 104. ” nebulosus CAND. Monogr. I, p. 232. Luçon, Leyte, Bojol, Mindanao orient.

brevipennis Cand. Ann. Soc. ent. Belg. Bull. 1875, p. 120.

Debyi Cand. Ibid. 1885 . . . . . . . . Sumatra occ. timoriensis Cand. Révision, p. 137 . . . . Timor. scitale Cand. Monogr. I, p. 228 . . ‘ . . I. Philippines Rosenbergi Cand. Révision, p. 138 . . . . Célèbes. oreas CAND. Élat. nouv. III, p. 19 . . . . . ” Semperi Cand. Ann. Soc. Belg. Bull. 1875,

p. 120 . . . . . . . . . . . Mindanao. lateralis CAND. Monogr. I, p. 227 . . . . Ceylan. lynceus CANd. Révision, p. 139 . . . . Hindoustan sept. nanus CAND. Ibid . . . . . . . . . . . Java or. putridus CAND. Monogr. I, p. 223 . . . . Java, Malacca. Lansbergei CAND. Élat. nouv. III, p. 19 . . . Java or. 
Doriæ Gestr. Ann. Mus. Gênes III, 1875,

p. 1000 . . . . . . . . Nouv. Guinée occ.

arfakianus GEstr. Ibid.

eryx CAND. Révision, p.140 . . . . Birmanie, Laos.

larvatus CAND. Ibid., p.141 . . . . . . . Chine.

tysiphone CAND. Ibid., p.142 . . . . . . Ceylan.

haje CAND. Ibid., p. 143 . . . . . . . . . Cambodge.

obliquus CAND. Ibid. . . . . . . Nouv. Guinée.

infumatus CAND. Ib., p. 144 . . . . . . " "

angularis CAND. Ibid., p. 145. . . . . . . Mysol.

basalis Schauf. Isis, 1864. . . . . Nouv. Guinée.

\section{3e Groupe.}

Australien.

gigas CAND. Monogr. I, p. 234 . . . . Cap York. Mac layi Cand. Tbid., p. 235 . . . . . . Clarence Riv. Gibboni Newm. Trans. ent. Soc., n.ser. IV, p.51. ('(l) Iork. prosectus CAND. Monogr. I, p. 236. . . . . Sydney. funereus CANd. Élat. nouv. I, p. 17 . . . Moreton Bay. suboculatus Cand. Monogr. I, p. 229 . . . Port Denison. funebris CAND. Ibid., p. 233 . . . . . . Moreton Bay. melancholicus CAND. Révision, p.147 . . Queensland. sericeus CaNd. Ibid., p.148 . Rockhampton, N. Guinée mér. pumilus CAND. Ibid.

\section{Le Groupe.}

Polynésien.

bituberosus Farrm. Ann. Soc. Belg., 1883 . . N. Bretagne. breviplicatus FAIRM. Ibid. . . . . . . ”

farinosus Montrodz. Ann. Fr., 1860, p. 255. Nouv. Calédonie. nivellus FAUveL, Bull. Norm., 1862.

costulicollis Fatrir. Ann. Soc. ent. Fr., 1881 . Firlij. IMontraveli Montrouz. Ibid., 1860 . . Nouv. Calédonie. cristatus Cand. Révision, p. 150 . . . . Nouv. Hébrides. 


\section{se Grompe.}

Américain.

Calais CAND.

tricolor Ox. Entom. II, p.31 . . . . . . St-Domingue.

nobilis SaLtí, Ann. Soc. ent. Fr., 1855, p. 263 . ” patricius CAND. Monogr. I,p 242 . . . Cuba.

luscus Ot. Entom. - Gerst. Wiegm. Arch.

primarius CAND. Élat. nouv. I, p. 18 . . . . ”

plebejus Cand. Révision, p. 152. . . . . Guyane.

griseus CAND. Élat. nouv. III, p. 20 - . Nouv.Grenade.

Nietoi Sallé. Revue Zool., 1870. . . . . . Mexique.

glaucus CAST. Silb. Rev. IV, p. 9 . . . . . Guyane. oculatus Lins. Syst. Nat. I, II, p. 651 . . . Etats-Unis. luscus FABR. System. Ent.

lusciosus Hope, Anim. Kingd, I, p. 363, 1832. Mexique. gorgops Lec. Journ. Acad. Phil., 1858, p. 35.

melanops Lec. New Sp. Col, 1863, p.83 . . Californie. naja CAND. Élat. nouv. I, p. 18.

myops FABr. Syst. Eleuth. II, p. 222 . . . Etats-Unis.

canadensis CAND. Élat. nouv. IV, p. 11 . . Canada.

\section{HEMIRHIPUS.}

Latr. Règne animal.

Antennes de douze articles longuement lamellés dans les deux sexes; corpś généralement velouté; grands.

lineatus FABr. Syst. Eleuth. II, p. 223 . . . Brésil. elegantissimus CAND. lat. nouv. III, 20 . . Uruguay.

var. Hougeti CAND. Élat. nouv. IV, p. 12.

Fairmairei Cand. Monogr. I, p. 248 . . . Amérique centr. Rojasi CAND. Ibid., p. 249 . . . . . . . . Vénézuéla. apicalis CAND. Ibid., p. 250 . . . . . . Nouv. Grenade. bimaculatus CAND. Ibid. . . . . . . Mexique. 
Bonvouloirei Cand. Révision, p. 154. . . Guyane. trilineatus CAST. Silb. Rev. IV, p. 12. . . . Brésil. quinquesignatus $\mathrm{C}_{\mathrm{AST}}$. Hist. nat. Ins. I, p. 234.

fascicularis FABr. Mant. I, p. 171. . Amerique intertropic. venosus CAst., loc. cit.

Dejeani Cand. Monogr. I, p. 253 . . . . Brésil.

decorus CAND. Ibid., p. 254. . . . . . . Mexique.

viduus Chevr. Ann. Soc. Fr., 1867, p.598 . . Chba.

Perroudi Cand. Révision, p. 155 . . . . . Brésil.

\section{Tetrigus.}

Cand. Monogr. I.

Antennes de onze articles, très courtes; leurs articles, ì partir du quatrième, portant une fine et longue lamelle insérée à leur base.

parallelus CAND. Monogr. I, p. 255. . . . Bengale. ater CAND. Tbid., p. 256 . . . . . . . . Ceylan. cyprius Batd. Berl. Zeitschr., 1871, p.50 . . Chypre. flabellatus GERM. Zeitschr. V, p.186. . . . J J pectinicornis Мотьсн.

Lewisi Cand. Mém. Soc.sc. Liége, 2" sér., V, p.6. Japon. grandis Lewis, Entom. Montl. May, 1879 . . 》 pexus CANd. Révision, p. 157. . . . Nouv. Guinée. Parryi CAND. Élat. nouv. I, p. 18 . . . . Natal.

Chalcolepis.

Carn. Monogr. I.

Canaux prothoraciques nuls; antennes de onze articles, simples, courtes. Forme des Chalcolepidius, mais mésosternum séparé du métasternum.

Luczoti CAnd. Monogr. I, p. 245. . Nicaragua, Véragua, Nouv. Grenade, Guyane. 


\section{TRIBU III.}

\section{CHALCOLÉPIDПTES.}

Mésosternum à bords élevés, confondus avec le métasternum, sans trace de suture entre eux. Téguments glabres ou écailleux, parés généralement de couleurs vives et variées. Taille grande.

\section{Chalcolepidius.}

Tarses sans lamelles, mandibules simples, aiguës, palpes courts:Corps généralement revêtu d'écailles diversement colorées, large de forme et très cambré. Américains.

\section{gection .}

Ecusson raccourci, plus ou moins bombé.

Haroldi CAND. Ėlat. nouv. II, 1878, p. 9 . . .

Pérou. Buckleyi JANs. ( ${ }^{1}$ ). Cisiula, 1882, p. 33 . . . Équateur. gossipiatus Guḱr. Rev. Zool., 1884, p. 18 Nouv. Grenade,

Vénézuéla.

oxydatus Cavd. Monogr. I, p. 266. Vénézuéla, Amér. centr. Le Moinei Caxd. Ibid., p. 265 . Nouv. Grenade, Vénézuéla. exulatus CANd. Révision, p.161. . . . . . Brésil sept. attenuatus Erichs. Zeit. f. d.Entom. III, p.86. Mexique. mexicanus CAST. Silb. Rev. IV, p. 13. . . . ” Mniszechi CAND. Élat. nouv. III, p. 21 . . . ” rubripennis Lec. Proc. Acad. Phil., 1861,p.336. Calif. mér. Lenzi Cand. Bull. Soc. entom. Belg., 1886, p.5. Cinaloa. Behrensi CAND. Ibid., p. 6. . , . . . . . ” Dugesi CAND. Ibid., p.6. . . . . . . Guanajuato. Jekeli CAND. Révision, p. 162. . . . . . . Guatémala. Desmaresti Chevr. Col. Mex. II, 1835, p. 195. Mexique. procerus Erichs. Mon, p. 84.

Rodriguezi Cand. Bull. Soc. ent. Belg., 1886 . Guatémala.

(') Cette espèce, que je n’ai point vue, me parait la même que la précédente. 


\section{Gection $\mathbf{I}$.}

Ecusson plan, déclive, plus long que large; antennes dentées dans les deux sexes.

sulcatus FABr. Entom. Syst. II, p. 220 . . . Antilles. validus CAnd. Monogr. I, p. 283. . . . . . 》 obscurus CASt. Silb. Rev.IV, p. 13 . . . ” longicollis Caxd. Monogr. I, p. 284 . . Nouv. Grenade. morio CAND. Ibid. . . . . . . . . . Oajuct. Perrisi Cand. Ibid., p. 285. . . . . Amérique équator. pruínosus Erichs. Zeit. f. d. Entom. III, p. 81. Mexique. Forreri CANd. Bull. Soc. Belg., 1886, p. 8 . . Xalisco. Silbermanni Chevr. Col. Mex. II, p. 197 . . . Mexique, Amérique centr. et équat. sericeus EnIchs. Monogr., p. 85.

Herbsti Erichs. Zeitschr.f. d. Entom. III, p. 82 . Amér. mérid. sulcatus Herbst, Käf.

virginalis CAND. Monogr. I, p. 288. . . . . Yucatan. chalcantheus CAND. Ibid. . . . . . . . Brésil. aurulentus CAND. Révision, p.183 . . . . Californie. circumductus CAND. Monogr. I, p. 289 . . Mexique. porcatus Linise, Syst. Nat. I, II, p. 652 . . . Amér. équat. guineensis, striatus. LINNÉ. Syst. Nat. pulvemilentus Herbst, Arch. VII.

Gandezei Dohre, Stett. Zeit., 1881 . . .Haut Amazonez. scitus CAND. Élat. nouv. IV, p. 12 . . . Vénézuéla. peruanus CAND. Bull. Soc. Belg., 1886 . . . Pérou. virens FABR. Mantiss. I, p. 172 . . . Amérique équator. Jansoni CAND. Révision, p. 165. . . . . Nicaragua. exquisitus CAND. Élat. nouv. IV, p. 13 . . . Véragua. limbatus Eschs. Thon Archiv. II, p. 33 . Amér. intertropic. porcatus OL. Entom. II. striatus FABR. System. Eleuth. Rostainei CAND. Élat. nouv. IV, p. 12. . . Colombie. 
zonatus Eschs. Thon Arch. II, p. 32 . . Amér. intertropic. smaragdulus Eschs., loc. cit.

approximatus ERICHS ('). Zeitschr. f. d. Ent.

III, p. 82

Mexique.

longipennis STURм, Cat., p. 67.

Mocquerysi CAND. Monogr. I, p.274 . . Nouv. Grenade. corpulentus CAND. Révision, p. 164 . . . . Bahia. Bomplandi GuÉr. Rev. Zool. VII, p. 17. . Nouv. Grenade. cyaneus Cand. Élat. nouv. III, p. 21 . . . . Brésil. Humboldti CAND. Ibid. . . . . . . . . Bogota. Fabricii Enichs. Zeitschr. III, p. 83 . . . Nouv. Grenade. Villei CAND. Élat. nouv., p. 9 . . . . . . . Équateur. erythroloma CAND. Monogr. I, p. 282 . . . Chiti. Albertisi CAND. Élat. nouv., p. 9. . . . . . Honolulu. Fryi Cand. Révision, p. 108 . . . . . . . Pérou. Eschscholtzi Chevr. Col. Mex. I, p. 7 . . . Mexique. Lafargei Chevr. Ibid.

Eschscholtzi var. Cand. Mon.

pistorius CAND. Bull. Soc. entom. Belg., 1890. Honduras. Lacordairei Cand. Monogr. I, p. 281. . . . Guatémala. inops CAND. Bull. Soc. entom. Belg., 1886 . . Chihuahua. rugatus CAND. Monogr. 1, p. 280 . . . . Guatémala. Juani CAND. Élat. nouv. IV, p. 13 . . . . . » mucronatus CAND. Ibid., p. 14 . . . . . . Colombie. angustatus CAND. Monogr. I, p. 290 . . . . Oajaca. Webbi Lec. ( $\left.{ }^{2}\right)$ Proc. Acad. Phil., 1854, p. $223 . \quad$ Californie.

\section{Gechion II.}

Antennes flabellées chez les mâles; écusson comme dans la $2 \theta$ section. Boucardi CAND. Révision, p. 169 . . . . Cuernavaca

(1) Pourrait n'être qu'une variété locale du précédent.

(2) Ne connaissant pas cette espèce, je ne sais s'il faut la rapporter à cette section. 


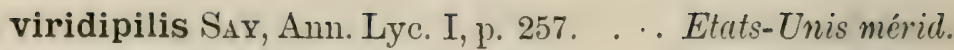
prasinus Erichs. Monogr., p. 85.

smaragdinus Lec. Proc. Acad. Phil., 1854,

p. 223.

\section{Senitotus.}

Eschs. Thon. Arch., 1829, II, p. 31.

Eucamptus Chevr., Pericallus Serv.

Tarses lamellés; corps ordinairement long, d'un jaune brillant variẻ de noir. Américains.

A Chaperon épineux.

a Une seule épine médiane . . . . . . Groupe I.

aa Deux épines au moins.

* Elytres sans bande noire ou trois (") au maximum.

* Une seule bande noire pour le prothorax. quand il en existe. . . . . . , Groupe II.

** Deux bandes noires. . . . . . . . Groupe III.

xx Plus de trois bandes sur chaque élytre.

* Six au maximum. . . . . . . . . Groupe IV.

** Plus de six. . . . . . . . . . Groupe V.

AA. Chaperon inerme.

a Une seule épine au sommet des élytres . . Groupe VI.

$\alpha \propto$ Elytres bi-épineuses ou tronquées au bout . Groupe VII.

\section{I ${ }^{\text {er }}$ Groupe.}

imperialis GuÉr. Rev. Zool VII, p. 15 . . Nouv. Grenade. cuspidatus Chevr. Col. Mex. I. . . Amérique centr. dorsalis Latr. Ann. Fr, 1834.

splendidus CAND. Élat. nouv. III, p. 23 . . . Véragua.

(1) Il va sans dire que ce groupentent est tout artificiel et ne sert que pour faciliter la détermination des espieces. Le genre Scmiotus est d'une telle homogéncité qu'il ne souftre aucun sectionnement naturel, si l'on excepte l'armature du front. 
2 Groupe.

suturalis FABR. Entom. Syst. II, p. 224 . . . Guyane. angulatus Drury, Tllustr.

sanguinicollis Blanch. Voy. d'Orb., Col. 128. Pérou, Bolivie. speciosus Erichs. Wiegm. Arch., 1847.

approximatus CAND. Monogr. I, p. 305 . . Guyane.

decoratus CAND. Ibid., p. 306. . . . . . . ”

furcatus FABR., loc. cit. . . . . . . . . »

fulvicollis Blanch. Voy. de d'Orb., Col. 129. Brésil, Bolivie.

Jansoni CAxd. Révision, p. 174. . . . . Équateur. bilineatus CAND. Monogr. I, p. $308^{\prime}$. . . . » fascicularis CANd. Ibid., p. 309 . . . . Nouv. Grenade. Dohrni CAND. Élat. nouv. IV, p. 14. . . . Panama. Badeni SteinH. Col. Heft. XIV, p.111 . . Nouv. Grenade. nigriceps CAND. Monogr. I, p. 319 . . . . ” limatus CAND. Élat. nouv. $\mathrm{TV}$, p. 15 . . . . Amazones. carus JANs. Cistula III, p. 34. . . . . . Équateur. Fryi CAND. Révision, p. 175 . . . . . . . ” insignis CAND. Monogr. I, p. 311. Amér. centr., Mexique mér. Var. longus CAND. Tbid., p. 315.

caracasanus RoJas, Rev. Zool., 1855, p.160 Vénézuéla. zonatus CAND. Révision, p. 176 . . . . . Équateur. chontalenus CAND. Ibid., p. 177. . . . . Nicaragua.

\section{Groupe.}

tæniatus Erichs. Ins. peruan, 1847, p. 75 . . Équateur. illustris CAND. Monogr., p. 313 . . . . . . Guyune. intermedius Herbst, Käf. X, p. 8. . . . . Brésil. cornutus KIRBY.

affinis GuĖr. V. d'Osculati, 1855 . . Équateur, Mexique. Lafertei CAND. Monogr. I, p. 313 . . . . . ” Candezei Krasch (1), Berl. Zeit., 1866, p. 180 . N. Grenade.

(1) Cette espèce, de mème que les deux qui précèdent, peuvent être considérées comme des formes locales de l'intermedius. 
angusticollis Blanch. V. de d'Orb., col. 127.

Brésil. convexicollis Blanch. Ibid., p. 128 . : . .

Bolivie ligatus CAND. Élat. nouv. IV, p. 15. . . . . Colombie

\section{Groupe.}

regalis Gukr. Rev.Zool, 1881, p. 16. N. Grencude, Équateur, Pérou. hispidus CAND. Élat. nouv. IV, p. 15.. . . . Vénézuéla. virgatus Erichs. Ins. peruan, 1847 . . . . Pérou. Illigeri Gútr. Rev. Zool., 18t4, p. 16 . . . Nouv. Grenade. Germari Gúkr. Ibid.

punctatus CAND. Monogr. I, p.322 . . Vénézuéla. Sommeri CAND. Ibid., p. 325 . . . . . . Nouv. Grenade. superbus Krasch, Berl. Zeitschr., 1866, p. 181. N. Grenade. acutus CAND. Révision, p. 180 . . . . . Équateur. bispinus CAND. Ibid.

)

multifldus CAND. Ibid., p. 181 . . . . . Colombie. trilineatus CAND. Monogr. I, p. $32 t$. . . Nouv. Grenade. elegantulus CAND. Ibid., p. 325 . . . . ”

\section{se aroup:}

Linnei Gó́r. Rev. Zool., 1844, p. 17 . . . Nouv. Grenade. macer CAND. Élat. nouv. IV, p.14 . . . . Colombie. cristatus CAND. Révision, p.182 . . . . . » glabricollis Cand. Monogr. I, p. 326. . . Nouv. Grenade. Reaumuri CAND. Ibid., p.327. . . . . ” Buckleyi CAND. Révision, p. 183 . . . . Équateur. punctato-striatus CAND. Monogr. I, p. 328 . N. Grenade. auripilis CAND. Révision, p. 184. . . . . . Bolivie.

G Groupe.

distinctus Herbst, Käf. X, p. 5, 21

Brésil. inermis KrRBy. 
ligneus LrNNÉ, Syst. Nat. II, p.652 . . . . Guyane. conicus Voet, Col. xylinus Perty, Del. Anim.

striatus GuÉr. Voy. d'Osculati, 1855 . . . . Équateur. Schaumi GuÉr. Rev: Zool. VII, p. 17. . . Nouv. Grenade. singularis KIrscr, Berl. Zeitschr., 1883, p.147. Équateur. pectitus CAND. Flat. nouv. IV, p. 15 . . . . Colombie. seladonius Gú́r. Rev. Zool. VII, p. 16 . Nouv. Grenade. virescens CAND. Monogr. I, p. 331. . . . . ” fusiformis KIRsch, Berl. Zeitschr., 1886,p. 181. Borrei CAND. Élat. nouv. II, p. 9. . . . . . Équateur. scitulus Cand. Révision, p. 186 . . . . . . . ”

\section{ge Groupe.}

luteipennis Guḱr. Mag. d. Zool., 1838 . . . Chili. formosus JiNs. Cistula III, p. 34 . . . . Équateur. brevicollis CAND. Monogr. I, p. 332 . . . Nouv. Grenade. quadricollis KIrsch, Berl. Zeit., 1886, p.181 . ” pulchellus CAND. Élat. nouv. IV, p.16 . . . ” juvenilis CAND. Révision, p. 187 \% . . . . Colombie. supplicans Krrsch, Berl. Ent. Zeitschr., $1883 . \quad$ ” stramineus CAND. Monogr. I, p. 333 . . . Nouv. Grenade. conicicollis CAND. Ibid. . . . . . . . . . » capucinus Cand. Ibid., p. 334 . . . . . . ” ” carinicollis KIRSCH, Berl. Ent.Zeit.,1883, p. $48 . \quad$ ” nigricollis CAND. Monogr. I, p. 335 . . . . ” æneovittatus KIRSCH, Berl. Ent. Zeitschr.,
1883, p. 48 . . . . . . . . . . . . . Equateur. quadrivittis Steinh. Col. Hefte XIV, p.113. Nouv. Grenade. diptychus CAND. Révision, p.188 . . . . Équateur. morio CAND. Monogr. I, p. 336 . . . . Nouv. Grenade. limbaticollis CAND. Ibid. . . . . . . . » 
Orstus.

CAND. Monogr. I, p. 338.

Chalcolépidiites à tarses simples et mandibules tronquées.

cacicus Cand. Monogr. I, p. 339

Orizaba. stenosomus CAND. Ibid.

SEMTоторSis.

Cand. Notes Leyd. Mus. IX.

Tarses lamellifères: mésosternum distinct du métasternum, ongles bidentés.

ungulata CAND. Notes Leyd. Mus. IX . . Nouv. Grenade.

\section{Canipsosternus.}

Chalcolépidiites des Indes orientales; grands, brillants, de teinte généralement verte et métallique, plus ou moins glabres; à palpes maxillaires longs; tarses non larellés; écusson arrondi.

A. Prothorax rouge latéralement.

splendidus Herbst, Käf. X, p. 3 . . . . . Ténassérim.

Hopei Westw. Cab. or. Entom.

Dohrni Westw. Cab. or. Entom., 1848, p.17 . Annam.

Mouhoti CAND. Révision, p. 191. . . . . . Laos.

gemma CAND. Monogr. I, p. 344. . . . . . Shang-Haï.

Lansbergei CAND. Élat. nouv. III, p. 23 . . . Java.

Stephensi Hope, Zool. Miscel., 1831, p. 25 . . Bengule sept. æneus CAND. Révision, p. 192 . . . . . . ”

B. Prothorax sans taches rouges.

a. Glabres.

aurat us Drurx, Ill. exot. Ins., 1773, II, p. 65 .

Chine.

fulgens FaBr., OL., CAND.

latiusculus CAND. Révision, p. 193 . . . Malacca. 
punctatus CAND. Tbid., p. 194 . . . . . . Singapore. rutilans Chevr. Rev. Zool., 1841, p. 222. . . Philippines. var. sumptuosus Hope. Trans., 1843. igneus CAND. Révision, p. 195 . . . . . Malacca. tæniatus CAND. Élat. nouv. II, p. 24. . . . Java. violatus Germ. Zeitschr. IV, p. 106 . . . Bengale. Plutus CANd. Révision, p. 195 . . . . . Bornéo. Mammon CAND. Élat. nouv. II, p. 10 . . . Birmanie. carinatus CAND. Élat. nouv. III, p. 24. . . . Bornéo. aureolus Hope, Griff. Anim. Kingd. . . Poulo-Pinang. Bohemani CAND. Monogr. I, p. 348 . . . . Ceylan. Templetoni Westw. Cab. or. Entom., p. 71 Moricei FaIrM. Ann. Soc. Fr. 1878, p. 272 . . Cochinchine. Duponti Hope, Trans. ent. Soc. Lond. III, p. 290. . . . . . . . . . . . Malabar. foveolatus Germ. Zeitchr. IV, p. 107.

Iris Cand. Révision, p. 196 . . . . . . . Madras. $b$. Plus ou moins pubescents.

rosicolor Hope, Trans. ent. Soc. Lond. III, p. 290 . . . . . . . . . . . Java. Latreillei GuÉr. Monogr. I, p. 351. . . . . Coromandel. Guerini CaND. Monogr. I, p. 351.

venustulus Cand. Ibid. . . . . . . . Ceylan. Nietneri CAND. lilat. nouv. I, 1865, p. 19. . . ” malaîsianus CAND. Ibid., p. 19 . . . . Malacca, Siam. pallidipes CAND. Monogr. I, p. 352. . . . . Ceylan. Mulleri Cand Révision, p. 198 . . . . . . Sumatra. Apollo Cand. Ibid.,p. 199 . . . . . . . . Laos. luctuosus CAND. Ibid . . . . . . . . . Madras. Gandezei Doнr, Stett. Zeits. 1873, p. 17 . . Bornéo. Delesserti Guḱr. Rev. Zool. 1840, p. 38. . . Nilgherries. Guerïi Hope, Trans. Ent. Soc., 1843.

Latreillei Germ. Zeitsch. IV, p. 101. brunneicornis CaND. Elat. nouv. IV, p. 16. . Darjeelinh. 
Cantori Hope, Trans. Soc. ent. Lond. III, p. 288 .

Birmanie.

Mniszechi CAND. Révision, p. 201 . . . . Malacca. sobrinus CAND. Ibid., p. 201 . . . . . . . Cochinchine. argentipilis CAND. Ibid., p. 202. . . . . . Siam. Saundersi CAND. Ibid., p. 203 . . . . . . Laos. parallelus CAND. Ibid., p. 203 . . . . . Birmanie. smaragdinus Hope, Frans. Ent. S. Lond. III. Madras. Proteus Hope, Ibid. . . . . . . . . . Manille. Leachii Hope, Ibid. . . . . . . . . Singapore. Wilsoni Hope, Ibid. . . . . . . . . . Madras. Eschscholtzi Hope $\left.{ }^{(}\right)$, Ibid. . . . . . . Manille.

TRIBU IV.

\section{OXYNOPTÉRITES.}

Mandibules saillantes, simples, aiguës, recourbées en forme de tenailles; antennes longuement flabellées chez les mâles; mésosternum et métasternum distinots; tarses sans lamelles.

\section{Oxynopterus.}

Hope. Proceed. Zool. Soc. 1842, p. 77.

Tournure des Campsosternus; non métalliques; épimères métathoraciques très larges; articles lamellifères des antennes plus longs que larges; indiens.

mucronatus OuIv. Journ. d'Hist. nat. I, p.262. Malaisie, Moluques.

Cumingi, javanus Hope, loc. cit . . . . I. Philippines. Andouini Hope, loc. cit. . . . . . . . . Bornéo. Harmsteni Cand. Notes Leyd. Mus. VII, p. 120. Sumatra.

(1) Ces cinq dernières espèces me sont inconnues et les noms font peut-être double emploi. 


\section{LEPTOPHYLLUS.}

Hope, Proceed. Zool. Soc., 1842, p. 79.

Elasmocerus Bohem. Megalorhipis. Lac. Cand.

Les 4 premiers articles lamellifères des antennes plus épais que longs.

Représente les Oxynoptérites en Afrique.

Strachani Hope, loc. cit., p. 78. Afrique intertrop., Congo.

latipennis Hope, validicornis. BoH, Lac, Cand. minor CAND. Monogr. I, p. 360 . . . . . . ”

\section{Pectocera.}

Hope, Proceed. Zool. Soc., 1872, p. 79.

Épimères métathoraciques petites. Asie.

Gantori Hope, loc. cit. . . . . . . . Haut Bengale. Mellyi Hope, Ibid. . . . . . . . . . Simla. malaïsiana CAND. Élat nouv. III, p. 24 . . Malaisie. Fortunei CANd. Mém. Sc. Liége, 2e sér., V, p. 6 Japon. Messi CAND. Révision, p. 207. . . . . . . Canton. brevicollis CAND. Élat. nouv. II, p. 10. . . . ”

\section{TRIBU V.}

\section{TÉTRALOBITES.}

Tarses quadrilamellés; corps massif, de très grande taille pour la plupart, de couleur uniforme. Insectes africains et australiens.

\section{Tetralobus.}

Servilue. Encyclop. méth. X, 1825.

Charitophillus Lad. Phyllophorus Hor.

flabellicornis LinNé, System. Naturæ . Afrique intertrop. albicans CAND. Monogr. 
soricinus CAND. Ibid.

cinereus Gory. Ann. Fr., 1832.

Livingstonei Caxd. Elat, nouv. III, p. 25 .

Zumlèze.

striatus CAND. Elat. nouv. IV, p. 16

)

subsulcatus Gér. Voy. de Lefebrre, p. 278. Abyssinie. Hopei GuÉr., loc. cit.

)

mystacynus CAxd. Monogr. I, p. 372.

Sénégal.

Duponti Hope. Proc. Zool. Soc., $18+2$.

Bas Congo,

Guinée infér.

Chevrolati CANd. Monogr. I, p. 374

Guinée. Savagei Hope?

Macari CAND. Élat. nouv. IV,p.17 . . Afrique centr. rotundifrons GuÉr. Voy. Lefebr., p. 282 . Afrique orient. heros BoH. Ins. Caffr.

cavifrons FATRM. Ann. Fr., 1887, p.149. . . Somalis. Rondani Bertolon, Nova Comm. Ac.Bonon.

X, p. 423 . . . . . . . Mozambique, Cafrerie. Polyphemus Bон. Ins. Caffr.

natalensis CAND. Monogr.

Goryi Hope, Proc. Zool. Soc., 1842. . . . . Abyssinie. punctatus CAND. Monogr. I, p. 377 . . . . Sennaar. sennaariensis CAND. Ibid., p.378 . . . . . »

Parryi Hope?

Raffrayi CAND. Élat. nouv. III, p. 26 . . . Abysinie. auricomus Hope, Proc. Zool. Soc., 1842. . . Gabon. rubiginosus CAND. Ẻlat. nouv. III, p. 25 . . Monrovia. cribricollis WAterh. Proc. Zool. Soc. Lond., 1888, p. 86 . . . . . . . . Afrique équat. or. Grandidieri CAxd. Élat. nouv. IV, p. 17 . . Madagascar. insularis CAND. Ibid., p. 18 . . . . . . . ”

Dohrni CAND. Élat. nouv. III, p. 26. . . . Guinée gigas FABr. Syst. Eleuth. II . . . . . Sénégambie. Dufouri CANd. Monogr. I, p. 378 . . . . Guinée.

Schuckardi? Hope. 
sobrinus CAND. Élat. nouv. IV, p. 17 . . . . ”

Mechowi Qued. Deutsch. entom. Zeits., $1886 . \quad$ Congo.

bifoveolatus Вонем. Ins. Caffr. I, II, p. 380 . Cafrerie. australasiæ Gory, Ann. Fr., 1836, p. 513 . . Swan River. Manglesi Hope, loc. cit.

Murrayi CAND. Monogr. I.

Albertisi CAND. Ann. Mus. Gênes XII, p. 108. Somerset. Fortnumi Hope, Proc. Zool. Soc., 1842 . . . Adélaide.

cylindriformis CAND. Élat. nouv. I, p. 20.

corrosus CAND. Élat. nouv. II, p. 10 . . . . Cap Curtis. capucinus CAND. Élat. nouv. III, p. 27 . . . Somerset.

\section{TRIBU VI.}

\section{DICRÉPIDUTES.}

Front caréné transversalement en avant; $2^{\circ}$ et $3^{e}$ articles des tarses lamellés, le $4^{\theta}$ petit et simple; écusson non cordiforme.

\section{Piezophyllus.}

Hope, Proc. Zool. Soc., 1842, p. 76.

Hanches postérieures élargies extérieurement; antennes simples, courtes.

Spencei Hope, loc. cit. . . . . . . . . Sénégambie.

Coresus.

(DEJ.). Gen. ineditum.

Piezophyllus pars Hope, CAND.

Hanches postérieures élargies extérieurement; antennes longues, à articles 3-10 larges.

macrocerus CAST. Silb. Rev., 1836 . . . . Madagascar. robustus Hope, loc. cit. firmus DeJ. in litt. 


\section{EMmitus.}

Latr. Ann. Fr., 1834, p. 157.

\section{Eucnemis ManNerH.}

Dicrépidites à tarses courts, les trois premiers articles munis de lamelles peu saillantes; corps trapu.

gigas Mannerh. Monogr. Eucnem, p. 14. . .

\section{OLOPHEUS.}

\section{Cand. Monogr. $\Pi$, p. 15.}

Diprépidiite à tête très bombée; les palpes terminés par un article linéaire.

gibbus CAND. Monogr. $\Pi$, p.15 . . . . . .

Gabon.

\section{Pantolamprus.}

Cand. Monogr. II, p. 16.

Mésosternum à bords verticaux; teintẹs brillantes.

perpulcher Westw. (Ampedus) Annals and

Magaz. 1812 . . . . . . . . . Guinée.

Varietates: auripennis, Savagei, Iris, cyanicollis, cyanocephalus Hope.

nitens CAND. Monogr. II, p. 18 . . . . . Gabon. auratus CAND. Notes Leyd. Mus. IV, p. 158. Libéria. Dohrni CAND. Élat. nouv. III, p. 27 . : . . ” rufipes Harold, Munch. Ent., 1878, p. 105 . . Congo.

\section{Psephus.}

Cand. Monogr. ПI, p. 19.

Ischiodontorum africana pars CAND., loc. cit.

Troisième article des antennes généralement plus court que le quatrième; hanches postérieures étroites. De l'ancien continent et spécialement de l'Afrique où ils forment la masse des Dicrépidiites. 
Especes africaines (').

puncticollis Вонем. Ins. Caffr. I, II, p. $384 . \quad$ Cafrerie. beniniensis CAND. Monogr. II, p. 21 . . . . Benin. pedestris Gertжr. Beitr. Ins. Faun. Zanzibar. Zanzibar. guineensis CAND. Élat. nouv. III, p. 27. . . Guinée. striatopunctatus CAND. Monogr. II, p. 21 . . V. Calabar. elimatus CAND. Ibid. II, p. 22 . . . . . . ” semicastaneus CAND. Élat. nouv. IV, p. 19. Niams-Niams. macrophthalmus CAND. Monogr. II, p. 26. . V. Calabar. fulvipes Quedenf. Deutsch Entom. Zeitsch. 1886

Raffrayi CAND. Élat. nouv. III, p. 26 . . . . Zanguebar.

Stanleyi CaNd. Élat. nouv. IV, p.19 . . . . Congo.

algoensis CAND. Monogr. II, p. 24. . . . . Cafrerie. apiculatus Вонем. Ins. Caffr. I, II, p. 385 . . Gariep. bucculatus CAND. Monogr. II, p. 25 . . . . Mradagascar. Candezei Rits. Entom. Monthl. Mag. 1873-74, p. 223 . Congo. antennatus CAND. Élat. nouv. IV, p. 18. . Niams-Niams. cavifrons Erichs. Wiegm. Arch.1843, I, p. 224 Angola. ineptus CAND. (sub nomine granulatus) Élat. nouv. II, p. 13. . . . . . . . . . . . Zanzibar. tabidus CAND. Élat. nouv. III, p.31 . . . . Cafrerie. geminatus CAND. Élat. nouv. II, p. 11 . . . Abyssinie. seniculus Cand. Notes Leyd. Mus. II, p. 7 . . Bagamoyo. niliacus CAND. Élat. nouv. II, p. 13. . . . . Kordofan. solidus CAND. Élat. nouv. IV, p. 18. . . . Niams-Niams. ochropterus CAND. Monogr. II, p.28. . . . Sénégal.

(1) J'ai cherché à diviser les Psephus en deux sections, suivant la conformation des hanches postérieures qui sont tantòt presque linéaires, tantòt dentées au milieu, mais cette mesure est devenue impossible, maintenant surtout que les espèces vont en se multipliant de plus en plus. Entre les deux formes extrêmes on trnive tous les passages. 
Oberthuri CAND. Elat. nouv. III, p. 28 . . . Zanguebar. militaris CAND. Tbid .

viridanus Gyllent. in Schönh. Synon. Ins. Sierra Leone. ceneolus CAND. Élat. nouv. I, p. 20. capillatus CAND. Ibid. IV, p. 18. . . . . . Akem. Bradshawi CAND. Ibid., p. 20 . . . , . Zambèze. vulneratus CAND. Ibid., p. 20 . . . . . . Cap. IMechowi CAND. Ibid. III, p. 29. . . . . . Congo. nigricornis CAND. Tbid., p. 29 . . . . . . Zanguebar. correctus CAND. Ibid., p.32 . . . . . . Gabon. synaptoides QUED., loc. cit., 1886 . . . . . Cuango. juvenilis CAND. Élat. nouv. III, p.32 . . . . Gabon. minor CAND. Ibid., p. 33. melancholicus CAND. Ibid., p. 29

Cafrerie. semiflavus CAND. Ibid.IV, p. 19 . . . . Guinée. collaris Bонем. Ins. Caff. I, II, p. 395. . . . Limpopo. monachus CAND. Monogr. II, p. 120 . . . V. Calabar. granulatus CAND. Tbid., p. 121 . . . . . Sénéga!. somalius Fatrm. Ann. Fr., 1887 . . . . . . Somalis. russatus FATRM. Ibid. . . . . . . . . . ” brevipennis CAND. Monogr. I, p. 27 . . . . Guinée. ovalis CAND. Ibid. II, p.119 . . . . . . Sierra Leone. Murrayi CAND. Élat. nouv. III, p. 33 . . . . V. Calabar. valens CAND. Tbid. IV, p.21 . . . . . . Afr. orient. nitidus CAND. Ibid. III, p. 33. . . . . . V. Calabar. morio CAND. Tbid., p. 30 . . . . . . . Monrovia. athoides CAND. Ibid., p. 31 . . . . . . Angola. litigiosus Boнem. Ins. Caffr. I, II, p.386. . . Natal.

(1) Nom déjà appliqué à une autre espèce comprise primitivement dans le genre ischiodontus. 
bicolor CAND. Élat. nouv. IV, p.20 . . . . Cap. dentatus CAND. Ibid. III, p. 34 . . . . . Gabon. sulcicollis QUED., loc.cit., 1886 (Ischiodontus.). Cuango. castaneipennis CAND. Monogr. II, p. 29. . . Cap. rubrescens CAND. Tbid. . . . . . . . Sénégal. sanguinolentus CAND. Élat. nouv. II, p. 34 . Gabon. calbonga CAND. Monogr. II, p. 124. . . . . ” granulipennis CAND. Élat. nouv. II, p. $11\left(^{(1)}\right.$. Zanzibar. insignis CAND. Monogr. II, p.122 . . . . Natal. moestus CAND. Ibid., p. 119 . . . . . . . Sierra Leone. nubilus Kluq. Monatsb. Berlin. Acad., 1855,

p. 647. . . . . . . . . . . . . . Mozambique. parallelus Bонем. Ins. Caffr. I, II, p. 388 . . Natal. ursus CAND. Monogr. II, p.120 . . . . Sierra Leone.

Espèces orientales.

rufinus CAND. Élat. nouv. III, p. 35 . . . . Ceylan. nobilis CaND. Ibid. . . . . . . . . . . ” cyaneus CAND. Élat. nouv. II, p. 11. . . . . Himalaya. desertor CAND. Élat. Jap. in Mém. Liége, sér. II, V, p. 7 • . . . . . • . . • • Japon. philippinensis CAND. Élat. des Philipp. Bull.

Belg., p. 18, 122 . . . . . . . . . Philippines. lateralis CAND. Élat. nouv. III, p. 36 . . . . Sumbawa. subfuscus CAND. Tbid. . . . . . . . Ternate. papuensis CAND. Ibid., p. 35. . . . Nouv. Guinée.

Dicronychus.

CAst. Hist. Nat. Ins., I. 1840, p. 251.

Conformation générale et aspect des précédents, mais pas de lamelles

(') Au lieu de granulatus déjà employé par la rentrée de l'Isch. granulatus dans ce genre. 
aux tarses, ce qui constitue une exception dans la tribu; crochets bidentés; compris autrefois dans les Campylides.

lamellicornis FArn. Ann. Fr., 1887, p. 150. Afrique équat. Hacquardi CAND. Élat. nouv. III, p. 36 . . . Zanguebar. bifidicomis FatrM. Ann. Fr., 1887, 8 .

flabellicornis Farrm. Ann. Fr. 1887, p. 150 . Zanguebar. psephoïdes CAND. Élat. nouv. III, p. 37 . . . Cafrerie. senegalensis CAST. Hist. nat. Ins. I, p. 251. . Sénégal. serraticornis CAST. Tbid.

Candezei Dux. Bull. Soc. ent. Belge, 1890 .

Soudan. cruentipennis CAND. Monogr. IV, p. 511 . . Cafrerie. granulatus CAND. Élat. nouv. III, p. 37 . . . ” mandibularis CAND. Ibid. . . . . . . »

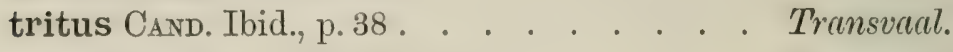
puerulus CAND. Ibid. IV, p. 21 . . . . . . Zanguebar. cinnamomeus CAND. Élat. nouv. I, p. 56. . . Bengale. ceylanicus CAND. Monogr. IV, p.512. . . . Ceylan.

\section{Tarsalgus.}

CAND. Élat. nouv. III, p. 39.

Mêmes caractères que les précédents, le premier article des tarses postérieurs gros, difforme.

tibialis Har. (Dicronyclues). Munch. ent. Ver., 1878, p. 106. . . . . . . . . Chango, Congo. Mechowi Cand. Élat. nouv. III, 1881, p. 39.

Thysi CANd. Ibid. IV, p. 21 . . . . . . Cassaï.

ANATHEsis.

CAND. Élat. nouv. I, p. 21.

Plusieurs articles des tarses lamellés; facies de Lacon. laconoides CAND., loc. cit. . . . Birmanie, Malacca. 


\section{HeterocrepidiUs.}

Guér. Mag. Zool. 1838.

Heteropus Germ.

Hanches postérieures inermes; articles 2 et 3 des antennes petits; mésosternum déclive; palpes terminés par un article ovalaire; jambes souvent élargies au bout.

crocipes Gerir. Ins. Sp. nov., p.41. . . . . Brésil.

gilvellus CAND. Monogr. II, p.34 . . . . . ”

granulatus CAND. Ibid. . . . . . . . . . »

picipes Germ. Zeitschr. I, p. 218. . . . . . „ „

columbicus SteinH. Col. Hefte XIV, p.114 . Médellin. ventralis GuÉr., loc. cit., 1838 . . . . . . Pérou. ænescens CAND. Monogr. I, p. 37 . . . . Ste-Catherine. puberulus Boнeм. Eugen. Resa, p. 66 . . 1. Gallapagos. rufus Steinh. Atti Soc. Sc. nat. XV, p. 566 . Rép. Argent. glis CAND. Monogr. II, p. 38 . . . . . . . Vera Cruz. depressus CAND. Ibid. . . . . . . . . . Brésil. ferrugineus Luc. Voy. de Cast. dans l'Amér. du Sud, 1857, p. 76 . . . . . . . . . . ”

tibialis CAND. Monogr. II, p. 39. . . . . . ” mendax CAND. Ibid. . . . . . . . . . castanopterus CAND. Ibid. . . . . . . Cap de B. Esp. afer CAND. Élat. nouv. IV, p. 22 . . . . . . Cafrerie.

Sossor.

Cand. Notes Leyd. Mus. V, p. 208.

Front allongé en pointe entre les antennes; la plaque nasale semielliptique.

Hageni CAND., loc. cit., p. 209 . . . . . . Sumatra.

SepHutus.

CAND. Ann. Mus. Gênes, XII, p. 108. 
Front très saillant, canaux prothoraciques droits, très grands et très protonds.

frontalis CAND, loc. cit. . . . . . Bornéo, Malacca.

\section{SPHENOMERUS.}

CAnd. Monogr. II, 1859, p. 41.

Articles 3-10 des antennes épaissis et comme tronqués au sommet.

antennalis CAND., loc. cit., p. 42 . . . . .

Ceylan.

submetallescens CAND. Ibid. . . . . . . »

canaliculatus Caind. Ibid., p. 43 . . . . ”

brunneus CAND. Élat. nouv. I, p. 22 . . . Siam.

Mouhoti CAND. Tbid., p. 21 . . . . . »

\section{Singhatenus.}

CaND. Monogr. П, p. 43.

Front coupé carrément en avant et portant une ligne médiane saillante.

tabrobanicus CAND., loc. cit. . . . . . Ceylan. rubiginosus CAND. Ibid. . . . . . . . . Hindoustan. Horsfieldi CAND. Élat. nouv. I, p. 22 . . . Bengale.

ELius.

CAND. Monogr. $\Pi$, p. 45.

Front arrondi en avant, très saillant, surmonté d'une ligne longitudinale; la plaque nasale haute.

prionocerus CAND., loc. cit., p. 45 . . . . . Madras. alveolarius $\mathrm{C}_{A \mathrm{ND}}$. Élat. nouv. $\Pi$, p. 11 . . Malacca. dilatatus CAND. Tbid. .. . . . . . . . » insularis CAND. Elat. nouv. IV, p.2 . . . Iles Andamans. umbilicatus CAND. Élat. nouv. I, p. 22 . . . Singapore. ventralis CAxd. Ann. Mus. Gênes, 1888, p. 673. Ténassérim. serraticornis Krrsch, Mittheil. Mus. Dresden, 1875, p. 7 . . . . . . . . Malacca. elegans Cand. Not. Leyd. Mus. II, p. 8 . . B Bornéo. 


\section{ADIAPHORUS.}

Cand. Monogr. II, p. 47.

Antennes longues, filiformes, hérissées; lamelles des tarses grandes. gracilicornis CAND. loc. cit. . . . . . Ceylan. ponticerianus CAND. Ibid. . . . . . . Pondichéry. punctatus Dum. Soc. entom. Belg. Bull., 1890. Hindoustan.

\section{Cyathodera.}

Buanch. Voy. de d'Orbigny, Col., p. 130.

Oxycleidius Eschs. Anoplischius CAND.

Antennes longues, marquées d'une ligne lisse longitudinale sur leurs deux faces; front creusé; le reste comme chez les suivants.

longicornis Blanch. loc. cit. . . . . . . Brésil. lanugicollis CAND. Monogr. II, p. 77 . . . . Guyane.

\section{ANoplischros.}

CAND. Monogr. II, p. 45.

Dipropus GERM. pars.

Antennes à $3 \Theta$ article de la taille du suivant, mésosternum déclive; hanches postérieures non dentées sur leur bord libre. Américains (sauf une espèce).

A. Antennes plus courtes que la moitié du corps.

corallinicollis CAND. Monogr. II, p. 53 . Amérique équinox. hemipyrrhus CAND. Ibid. . . . . . . Brésil. elegantulus CAND. Élat. nouv. III, p. 41. . . Bogota. pallidipes CAND. Monogr. II, p.54. . . . . Brésil. castaneipennis CAND. Ibid., p. 55 . . . . . ” acallus CAND. Ibid., p. 56 . . . . . . . Caracas. mus CAND. Tbid. . . . . . . . . . . . Mexico. vitticollis CAND. Ibid., p. 57 . . . . . . Colombie. æoloides CAND. Élat. nouv. III, p. 41 . . . . ” rufulus CAND. Monogr. II, p. 58. . . . . . Mexique. 
testaceus CAND. Ibid.

Colombie.

catulus CAND. Ibid., p. 59 . . . . . . . . Mexique.

melanotides CAND. Ibid., p.60 . . . . . Yucatan.

athooides Steinn. Col. Hefte XIV, p. 116 . Nouv. Grenade. ripurus CAND. Monogr. $\Pi$, p. 60. . . . . . »

atractodes CAND. Ibid., p.61 . . . . . . Mexique.

morosus CAND. Ibid. . . . . . . . . Colombie.

molliculus CAND. Ibid., p. 62 . . . . . . . »

bicarinatus CAND. Ibid. . . . . . . . . Brésil.

pilosus Cand. Ibid., p. 63 . . . . . . . . Bahia.

vestitus CAND. Ibid., p.63. . . . . . . Nouv. Grenade.

egaensis CAND. Ëlat. nouv. III, p. 40 . . . . Amazones.

hæmatopus CAND. Monogr. II, p. 64 . . . . Brésil.

clavus CANd. Élat. nouv. I, p.22. . . . . Guyane.

La Rochei Steinh. Col. Hefte XIV, p. 114. Nouv. Gienade. limatus CAND. Élat. nouv. IV, p. 22.

)

trapezicollis CAND. Monogr. II, p. 65.

Brésil.

rusticus CAND. Élat. nouv. III, p. 40 .

)

anthracinus CAND. Monogr. $\Pi$, p. 65.

Colombie.

B. Antennes très longues.

longulus CAND. Ibid., p. 66.

parallelus Steinh. Col. Hefte XIV, p. 117 . Nouv. Grenade.

Landolti Stenn. Munch. Ent. Ver., 1877, p.79. ”

conicicollis CaNd. Monogr. II, p. 66 . . . . »

longipennis CAND. Ibid., p. 67 . . . . . ”

lateralis CAND. Tbid. . . . . . . . Brésil.

suturalis CAND. Élat. nouv. III, p. 40 . . . . ”

hirtellus CAND. Monogr. II, p. 68 . . . . . ”

nigrinus CAND. Ibid., p. 69 . . . . . . Bolivie.

depressipennis CAND. Ibid. . . . . . Cuba.

sulcifrons CAND. Ibid., p.70 . . . . . Guadeloupe.

punctatus CAND. Ibid., p.70 . . . . . St-Domingue.

pyronotus CAND. Ibid., p.71. . . . . Cuba. 
venustus JACQ. Duv. Hist. Cuba, 1857, p. 70 .

Cuba.

seminiger CAND. Monogr.

sagranianus $\mathrm{J}_{\mathrm{ACQ}}$. Duv., loc، cit.

)

lineipennis CAND. Monogr.

ruficeps CAND. Élat. nouv. I, p. 23

C. Angles postérieurs du prothorax droits ou même recourbés en dedans, surmontés d'une forte carẻne.

laticollis Esch. Thon. Arch. II, p. 1,3 . . . Brésil. melanotiformis CAND. (emend.) Élat. nouv.

III, p. 41.

compressicornis Stennh. Col. Hefte XIV. Nouv. Grenade. attenuatus SternH. Col. Hefte XIV, p. 115. . ” ग ebeninus SteInн. Ibid. . . . . . . . . »

pubescens CAND. Monogr. II, p.75 . . Buenos-Ayres. transversus CAND. Ibid. . . . . . Nouv. Grenade. Glaudii CAND. Tbid., p. 76 . . . . . . . Guyane.

D. Espèce asiatique.

laminatus CAND. Ibid., p. 57

Ceylan.

\section{Ovipalipus.}

Sol. Gax, Hist. de Chile, 1851, p. 19.

Dernier article des palpes ovalaires; articles 2 et 3 des antennes petits; corps filiforme.

pubescens Sol., loc. cit.

Chili.

LoBoderus.

Gut́r. Magas. de Zool., 1831, p. 9.

Deux appendices pénicillés à la base du prothorax.

appendiculatus Perty, Del.Anim., 1830, p. 21.

Bahia.

monilicornis Gǘr. loc. cit. 


\section{Crepidius.}

CAND. Monogr. II, 1859, p. 80.

Articles 3-10 des antennes des mâles longuement appendiculés.

ophthalmicus CAND. Monogr. II, p. 81 . . Brésil.

flabellifer Erichs. Wiegm. Arch., 1847, I, p.77. Pérou. castaneus Blanch. Voy. d'Orb. col. 132. . . Corrientes. cuneiformis CAND. Monogr. II, p. 83 . . . . Guyane. resectus CAND. Ibid. . . . . . . . . . Brésil. emarginatus CAND. Ibid. . . . . . . » pubescens CAND. Ibid., p. 85. . . . . . . ” Saundersi CAND. Ibid. . . . . . . . Amazones. rhipiphorus CAND. Ibid., p. 86 . . . . Guadeloupe.

\section{SpILUs.}

CAND. Monogr. II, p. 87.

Bords de la fossette mésosternale perpendiculaire, en sorte que celle-ci regarde directement en avant.

atractomorphus CAND. Monogr. II, p. 88. . Brésil. nitidus Cand. Tbid. . . . . . . . . Guyane. rubidus CAND. Tbid., p. 89. . . . . . . . Brésil. brevis CAND. Élat. nouv. II, p.43 . . . . . Amazones. lævigatus CAND. Monogr. II, p. 89. . . . . Colombie.

IsCHODONTUS.

CaND. Monogr. II, 1859, p. 90.

Dicrépidiites américains caractérisés comme les Anoplischius, mais à hanches postérieures dentées au milieu de leur bord libre.

\section{section $\mathbf{I}$.}

Troisième article des antennes aussi grand que le quatrième.

puncticollis FABr. Syst. Eleuth. II, p. 234. . Brésil. auricomus CAND. Monogr. II, p. 97 . . . . ” vulpinus CAND. Ibid., p.98 . . . . . . Chiquitos. 
brunneus CAND. Tbid. : . . . . . . . Brésil. vicinus CAND. Ibid. . . . . . . . . . . nocturnus Stenнн. Col. Hefte XIV, p. 119 . Nouv. Grenade. vulpes StennH. Ibid.

pexus Germ. Ins. Sp. nov., p. 55. . . . . . Brésil. inornatus CAND. Monogr. II, p. 100 . . . . Colombie. piceus Stenn. Col. Hefte XIV,p.117. . . Nouv. Grenade. pinguis CAND. Monogr. II, p. 100 . . . . . Mexico. soleatus SAy, Trans. Am ph. Soc. VI, p. 176 . Texas. simplex Lec. Ibid. X, p. 462 . . . . . . . ” oblitus CAND. Monogr. II, p. 101.

ferreus LEC. loc. cit. )

Tricrepidius triangulicollis Мотsch. approximatus CAND. Monogr. III, p. 113 . . hawaïensis CAND. Élat. nouv. III, p. 42 . . Hawai. chloropterus ErIc. Schomb. Guyan. III, p. 558 Guyane. scutellaris SteInн. Col. Hefte XIV, p. 118. Nouv. Grenade. biemarginatus Stenn. Ibid. . . . . . . » rufolimbatus STEINH. Tbid. . . . . . . . ” smaragdinus CAND. Monogr. II, p. 102 . . . Mexique. viridipennis CAND. Ibid., p. 103. . . . . Nouv. Grenade. horripilosus CAND. Ibid. . . . . . . . . ” vulneratus CAND. Ibid., p.104 . . . . . Brésil. erythroderus CAND. Élat. nouv. II, p. 12 . . Guatémala. decoratus CAND. Ibid. III, p. 42. . . . . Brésil. nigricollis CAND. Monogr. II, p. 104 . . . . Montevideo. collaris CAND. Élat. nouv. II, p. 12. . . . . Bahia. Faldermanni CAND. Monogr. II, p. 105. . . Brésil. brasilianus Germ. Ins. Spec. nov., p. 55. . . ” fuscicornis CAND. Monogr. II, p. 106 . . . . ” magnicornis BL. Voy. d'Orb., p. 133. . . . Chiquitos. aper CAND. Monogr. II, p. 107 . . . . . St-Domingue. antennatus CAND. Tbid., p. 108 . . . . . Cuba. obscurus CAND. Ibid. . . . . . . . . Brésil. 
erosus CANd. Ibid., p. 109 . . . . . . . Brésil: acutangulus CAND. Ibid., p. 110 . . . . Bolivie. venustulus CAND. Ibid. . . . . . . . . Brésil. semipunctatus CAND. Ibid., p. 111 . . . . Ballia. atrocæruleus CAND. Ibid. . . . . . . . Mexico. cyanopterus CAND. Ibid., p. 112 . . . . . Veracruz. thoracicus CAND. Ibid., p. 113 . . . . . . Mexico. tuspanus CAND. Ibid.

) badius CAND. Tbid., p. 114 . . . . . . Nicaragua. sus CAND. Tbid. . . . . . . . . . . . Mexico. angustipennis CAND. Ibid., p. 115 . . . . . ” depressus CAND. Ibid. vetulus CAND. Ibid., p. 116. anceps $\mathrm{CAND}_{\mathrm{AN}}$. Tbid. nigricornis CAND. Ibid., p. 117 . . . . . . Veracruz. striatus CAND. Ibid. . . . . . . . Cuba. quadraticollis CAND. Elat. nouv. II, p. 12 . . Guatémala. fatuellus CAND. Monogr. II, p. 118 . . . . Paraguay.

\section{section $\mathbf{d}$}

Articles 2 et 3 des antennes petits.

latus CAND. Monogr. II, p. 124

Guyane. nigrita $C_{A N D}$. Ibid., p. 125 . Brésil. subsericeus CAND. Tbid., p. 126. Guyane. piceipennis Sternн. Munch. Ver., 1877, p. 80 . Ocania. comosus Germ. Zeitschr. I, p. 220 . Brésil. alopex FABR. System. Eleuth. II, p. 236 . )) brevicollis Stenvi. Col. Hefte XIV, p. 190. Nouv. Grenade. proximus CAND. Monogr. II, p. 128 . . . Ste-Catherine. punctatus CAND. Ibid. . . . . . . Guyane. deletus CAND. Ibid.

) vittatus Steinh. Munch. Ver., 1877, p. 80 . . Ocania. ichthidius CAND. Monogr. II, p.129 . . . Mexique. rufus CAND. Ibid., p. 130. 
fasciatus CAND. Ibid., p. 131.

cruciatus CAND. Ibid . . . . . . . . Nouv. Grenade. crocicollis CAND. Ibid. . . . . . . . . Colombie.

Trielasmus.

Blanch. Voy. d'Orb. VI, p. 11, 131.

varians $B u$. loc. cit.

Chiquitos.

Atractodes.

Germ. Zeitschr. f. d. Entom. I, p. 219.

Atractosomus Lad. Gen. IV. CAND. Monogr. II.

Bords de la fossette mésosternale élevés, horizontaux.

\section{Section .}

Troisième article des antennes plus petit que le quatrième.

auricomus CAND. Monogr. II, p.134 . . . Vénézuéla.

flavescens Germ. Zeitschr. I, p.'219 . . . . Brésil.

flavipes CAND. Élat. nouv. II, p. 13 . . . . ”

carinatus CAND. Monogr. II, p. 135 . . . . ”

conicicollis CAND. Tbid., p. 136 . . . . . . ”

cratonychoides CAND. Ibid., p. 137 . . . . ”

infumatus CAND. Tbid. . . . . . . . . . ”

dimidiatus Luc. Voy. Casteln., 1859, p.76 . . ”

\section{Section II.}

Troisième article des antennes aussi grand que le quatrième.

rubidus CAND. Monogr. II, p. 138 . . . . . Brésil. ferrugineus CAND. Ibid. . . . . . . . Nouv. Grenade. tabularius CAND. Ibid., p. 139 . . . . . Vénézuéla. rhomboïdalis CAND. Ibid. . . . . . . Guyane. luteipennis CAND. Elat. nouv. II, p.13 . . . Bahia. robustus CAND. Monogr. II, p. 140. . . . . Para. cayennensis CAND. Ibid., p. 141. . . . . . Guyane plebejus CAND. Ibid. . . . . . . . . . ” atricornis ERICHs. Schomb. Guyan.II, p. 558 . ” 
corax CAND. Monogr. II, p.142. . .. . . . Mexique. arcuatus CAND. Ibid.

DicRepidius.

Escrs. Thon. Arch., ПI, p. 31.

Bords de la fossette mésosternale élevés, horizontaux; antennes des mâles flabellées.

ramicornis Palis. De Beauv. Ins. I, p. 10 . Mexique, Antilles, Anér. mérid. pectinicornis EscHs., loc. cit.

corvinus CAND. Monogr. II, p. 145. . . . . Mexique. cavifrons CAND. Élat. nouv. III, p. 43 . . . Guadeloupe. palmatus CAND. Monogr. $\Pi$, p. 146 . . . . Amér. sept. thoracicus CAND. Tbid. . . . . . . . Colombie.

\section{TRIBU VII.}

\section{EUDACTYLITES.}

Front caréné transversalement en avant; tarses dilatés.

Achrestus.

CAND. Col. Heft. V. 1869, p. 122.

Anepsius CAND. Monogr.

Quatrième article des tarses petit, les trois premiers lamellés.

flavocinctus CAND. Monogr. $\Pi$, p. 157 . . . Brésil. marginatus CAND. Ibid., p. 73. (Anoplischius). ) saucius CAND. Tbid., p. 157 . . . . . N. Grenade. phyllocerus CAND. Ibid., p. 158. . . . . . Vénézuéla. lycidioides CAND. Ibid., p. 155 . . . . . G Guyane.

EUdactylus.

SALLE, Ann. Fr., 1854, p. 266.

30 et 4 articles des tarses dilatés et lamellés en dessous. 
costatus CAND. Monogr. II, p. 161 . . . .

Guyane.

albipes CAND. Ibid . . . . . . . . . . ”

alboguttatus CAND. Élat. nouv. I, p. 23 . . . ”

bifoveatus CAND. Ibid. III, p. 43 . . . . . ”

eques CAND. Monogr. II, p. 162 . . . . . . ”

interruptus Ourv. Journ. Hist. nat. I, p. 265 . ”

abdominalis Perty, Delect. anim., p. 21 . . ”

Grandini CAND. Élat. nouv. I, p. 23. . . . . Mexique

castus JANs. Cistula III, 1882, p. 36 . . . . Équateur.

Boucardi Sallí, Rev. Zool, 1873 . . . . . Oaxaca.

prodigus JANs. loc. cit., p. 35. . . . . . . Équateur.

carnifex CAND. Monogr. II, p. 164 . . . . . Bahia.

bicinctus CAND. Ibid., p. 165 . . . . . . . Brésil.

Wapleri SALLE, Ann. Fr., 1854, p. 267 . . St-Domingue.

dimidiatus CAND. Élat. nouv. II, p. 14 . . . Choco.

Schaumi CAND. Monogr. II, p.167. . . . . Cuba.

cyanipennis CAND. Tbid. . . . . . . . ”

discoidalis CAND. Élat. nouv. II, p. 14 . . . Cafrerie.

GLYPHEUS.

Cand. Monogr. II, p. 168.

Tarses à quatrième article seul dilaté-lamellé; métathorax caréné mésosternum horizontal.

villosulus CAND. Monogr. II, p. 169 . . . Austr. orient. Lansbergei CAND. Élat. nouv. III, p. 44. . . Victoria.

\section{Simodactylus.}

Cand. Monogr. II, p. 169.

Tarses simplement dilatés; mésosternum déclive.

cinnamomeus Borsd. Voy. Astrol., Col. $106 . \quad$ Océanie.

Chazali Le GumL. Rev. Zool.

subcastaneus FaIrm. Rev. Zool.

sericans FAIRM. Ibid. (sub nomine Monocrepidius). 
guttatus CAND. Ann. Genov. XII, p. 110 (Monocrepidius)

Ternate.

Tastui Le Guilu. Rev. Zool. 1844, p. 221.(Dicrepidius). Hamoa. similis Cand. Ann. Mus. Genov. XII, p. 111. Nouv. Guinée. tertius CAND. Élat. nouv. III, p. 44. . . . . "

fasciolatus FA1rm. Journ. Le Nat. Mars 1883. N. Bretagne. pulcherrimus $\mathrm{C}_{A N D}$. Élat nouv. IV, p. 23. 1. Philippines.

\section{Metanthoides.}

CANd. Élat. nouv. I, p. 24.

Tarses courts, à articles triangulaires, dilatés, le quatrième laminifère, le cinquième petit.

latimanus CAND. Loc. cit. . . . . Grand Bassam. Gestroi CAND. Élat. nouv. П, p. 14 . . . . Zanzibar. luteipes CAND. Tbid. III, p. 45 . . . . . . ” ligneus CAND. Élat. nouv. II, p.15 . . . . Malacca. nitidus Cand. Notes Leyd. Mus. II, p. 1. . Sumatra. Feæ CAND. Ann. Mus. Genov. 1888, p. 7 . . . Birmanie. relegatus CANd. Élat. nouv. IV, p. 23 . . . Bornéo.

\section{PAChyderes.}

Latr. Ann. Fr. 1834, p. 149.

Prothorax très dilaté, beaucoup plus large que les élytres.

ruficollis GuÉr. Icon. Règne anim., p. 41 . . Malaisie. coccineus CAND. Monogr. II, p.174 . . . . Népaul. apicalis CAND. Élat. nouv. I, p. 24 . . . . Malacca. macrothorax WIEDM. Zool. Mag. II, p. 1, 105 Java. minor CAND. Ibid. IV, p. 22 . . . . . Malucca. niger CAND. Élat nouv. II, p.14. . . . . . Hindoustan, Birmanie, Malaisie. bengalensis CAND. Ibid. IV, p. 23 . . . . Bengale. 


\section{TRIBU VIII. MONOCRÉPIDITES}

Tête un peu convexe et carrée, rebordée en avant; hanches postérieures fortement dilatées dans leur moitié interne en une grande lame arrondie; tarses comprimés, le quatrième article dilaté-cordiforme et généralement lamellé.

\section{GLYPHOCHUUS.}

Cand. Monogr. II, p. 180.

Labre échancré; sutures prosternales légèrement concaves ot creusées. furvus Erichs. In Wiegm. Archiv. 1842. . . Tasmanie. tasmanicus CAND. ( $\left.{ }^{1}\right)$ Monogr. II, p. 181. . . ” lucidus ErIchs, loc. cit. . . . . . . . . ” leptus CAND. Monogr. II, p. 182 . . . . . . Swan River. occidentalis CAND. Élat. nouv. III, p. 45. . . ” Ghampioni CaNd. Ibid. . . . . . Champion Bay. bicolor Cand. Ibid. II, p. 15 . . . . . . . Sydney.

\section{DORYGONUS.}

Cand. Monogr. II, p. 182.

Labre entier; sutures prosternales concaves et creusées. stygius CAND. Monogr. $\Pi$, p. 183 . . . . Madagascar. xanthobracus CAND. Ibid., p. 184. . . . . ” famelicus CAND. Ibid. . . . . . . . . . ” semiustus CAND. Tbid., p. 185 . . . . . . ” amaurus CAND. Ibid., p. 186. . . . . . . ” Coquereli Cand. Tbid. . . . . . . . . . ' ’

Apochresis.

CAND. Élat. nouv. III, p. 46.

Caractères des Dorygonus, avec les sutures prosternales fines et droites.

asper CAND. Loc. cit. . . . . . . Benguela.

( $\left.{ }^{1}\right)$ Peut-être la mème espèce que la précédente, que je ne connais pas. 


\section{Telesus.}

Cand. Notes Leyd. Mus., II, p. 9.

Hanches postérieures rectangulairement dilatées en dedans; cinquième article des tarses très petit, ses ongles exigus.

Ritsemæ CAND., loc. cit.

Congo.

\section{Phedomenus.}

CAND. Élat. nouv. IV, p. 23.

Caractères des Monocrepidius, à dernier article des tarses très réduit; habitat spécial.

venustus CAND. Ibid. IV, p. 24 . . . . . Madagascar. decoratus CAND. Ibid . . . . . . . ” bellus CAND. Ibid . . . . . . . . . . »

\section{MoNocRePIDIUS.}

Eschs. Thon, Arch. II, 1, p. 31.

Front bombé légèrement, ou plat, plus ou moins carré, rebordé on avant; quatrième article des tarses lamellé; banches postérieures fortement élargies en dedans en une expansion non anguleuse.

NотA. Ce genre étant très nombreux en espèces réparties dans toutes les régions chaudes du globe, je le divise en sept groupes, basés en partie sur la situation géographique. Il est inutile de faire remarquer que ce groupement n'est fait que pour faciliter l'arrangement des espèces.

Amérique ( ${ }^{1}$ ).

a. Corps allongé; élytres longues, atténuées en arrière, épineuses au bout . . . . . Groupe I.

aa. Corps plus ou moins large; élytres inermes au bout.

b. Articles 2 et 3 des antennes très petits et égaux, plus courts réunis que le quatrième.

$b b$. Article 3 des antennes plus grand que le second, parfois égal au quatrième. . . . Groupe III.

(1) Je commence par l'Amérique, parce que c'est là que les espèces sont le plus nombreuses el le plus remarquables. 
Autres pays.

Australie orientale et méridionale y compris la

Tasmanie . . . . . . . . . . Groupe IV.

Australie de l'Ouest. . . . . . . . . . . Groupe V.

Polynésie, Nlle Guinée et Iles voisines. . . . . Groupe VI.

Asie et Malaisie. . . . . . . . . . . Groupe VII.

\section{er Groupe.}

Ce groupe correspond aux espèces du genre Conoderus EsCHs.

alacer CAND. Élat. nouv. III, p. 47 . . . . Brésil.

fuscofasciatus Eschs.THоN, Archiv. I, p. 138.

Brésil, Rép. Arg.

pugionatus Germ. Zeitschr. I, p. 223.

bigatus GERM. Ibid. . . . . . . . . . . ” ”

fulvus CAND. Élat. nouv. II, p. 16 . . . . . Brésil.

leucophæatus CAND. Monogr. II, p. 201 . . ”

rufescens CAND. Ibid. . . . . . . . Vénézuéla.

rufidens FABr. System. Eleuth. II, 1, p. 234 . Brésil.

discolor Eschs. Thon, Arch.; Cand. Monogr.

lateralis Eschs. Tbid.

angulatus StenNH. Col. Hefte XIV, p. 121 . . N. Grenade. Rodriguezi CAND. Élat. nouv. III, p. 47 . . . Guatémala. spinosus Eschs. Entomogr., 1822, p. 65. . . Brésil. malleatus GerM. Ins. Sp. nov., p. 50 . . . . ”

formosus CAST. Hist. Nat. II.

sinaloæ CAND. Élat. nouv. IV, p. 25 . . . . Mexique. calcaratus CAND. Ibid. III, p. 49 . . . . . Brésil.

2 Groupe.

a. Elytres généralement mouchetées de noir indépendamment d'autres taches. Grandes espèces, en général.

dimidiatus GERM. Zeitsch. I, p. 230 . . . .

Brésil. rubrescens Bu. Voy. d'Orb. Col., p. 136 .

Germari Bонем. Res. Eugen. p. 67. . . Prov. Argentines. insignis CAND. Monogr. II, p. 214. 
melanurus CANd. Monogr. II, p. 215 . . . Brésil. abdominalis CAND. Ibid., p. 217. . . . . . " inquinatus CAND. Ibid., p. 218. sticticus Erichs. Ins. per.in Wiegm. Arch. 1847, p.78. . . . . . . . . . Pérou. stigmosus Germ. Zeitschr. I, p. 230 . . . . Brésil. tactus Germ. Tbid. . . . . . . . . . . » lenis CAND. Élat. nouv. III, p. 48. . . . . . Para. scalaris GerM. Insp. Sp. nov., p. 47 . . . . Brésil mér. Rép. Arg., Patagonie. Azaræ CANd. Élat. nouv. IV, p. 25 . . . . . Paraguay. columbinus CAND. Monogr. II, p. 222. . . . Colombie. nocturnus CAND. Ibid. . . . . . . . M Mexique. fossulatus CAND. Élat. nouv. III, p. 48 . . . Brésil. apiatus EnICHs. Wiegm. Arch., 1847, p. 78. . Pérou. Pilatei CAND. Monogr. II, p. 224 . . . . Yucatan. hypolithus CAND. Ibid. . . . . . . Vénézuéla. concretus CAND. Élat. nouv. III, p. 50. ... Guyane. xysticus CAND. Monogr. II, p. 225 . . . . . Texas.

$b$. Élytres avec ou sans taches, mais sans mouchetures noires.

semimarginatus Latr. Voy. de Humboldt II, 1833, p. 16 Colombie. impluviatus Germ. Zeitschr. I, p. 225. Brésil. cuniculus CAND. Monogr. II, p. 209 ) abbreviatus Germ. Zeitschr. I, p. 229 . Botivie. flavovittatus Bu. Voy. d'Orb.

Brésil.

var. Lacordairei Des. Cat.

pertusus CAND. Élat. nouv. II, p. 16

Mendoża. quadriplagiatus CAND. Élat. nouv. IV, p. 25. lacertosus CAND. Monogr. II, p. 215 .

Brésil. repandus Errchs. Wiegm. Arch., 1847, I, p.78. confusus BL. Voy. d'Orb. Col., p. 135. 
ternarius Germ. Zeitschr. I, p. 229 . . . . Brésil. vulneratus CAND. Élat. nouv. III, p. 48 . . . Mexique. capistratus GERM. Zeitschr. I, p. 226 . . . Brésil. molitor CAND. Monogr. II, p. 205 . . . . . Para. Baleni Stenn. Col. Hefte XIV, p. 122 . . Nouv. Grenade. decimus Cand. Monogr. П, p. 206 . . . . Vénézuéla. rufipes CAND. Ibid., p. 217 . . . . . . . Bahia. pinguis CAND. Ibid., p. 207. . . . . . . . Antilles. longicornis CAND. Tbid. . . . . . . . Nouv. Grenade. ingenuus $\mathrm{C}_{\triangle \mathrm{ND}}$. Élat. nouv. IV, p. 25. . . . Uruguay. nubeculosus CAND. Ibid., p. 26 . . . . . Équateur.

\section{Groupe.}

figularis CAND. Élat. nouv. III, p. 50 . . . St-Domingue. lividus DE GEER, Mém. Ins. IV, p. 162. . . . Antilles. castanipes, dispar HerBst, Käf. elongatus BEAUv. Ins. Afr.

lobatus SAY, Journ. Acad. Phil. testaceus OL. Entom.

albinus CAND. Monogr. II, p. 246 . . . . . Mexique. parallelus CAND. Ibid. . . . . . . . . ” curvifrons CAND. Ibid., p. 247 . . . . . St-Domingue. sericatus CAND. Ibid., p. 249. . . . . . . ” bifoveatus Beauv. Ins. Afr. et Am., p.78 . . Cuba. var. castanipes GERM. Zeitschr. I, p. 228. sericans Chevr. (pour sericatus) Ann. Soc. Fr. 1867, p. 604. memorabilis CAND. Monogr. II, p. 249 . probus CAND. Élat. nouv. III, p. 50 . . . . . Guatémala. tarsalis CAND. Monogr. II, p. 250 : . . . . Mexique. intermissus KтrScH, Berl. Zeitschr. 1873 . . Pérou. flavangulus CAND. Monogr. II, p. 250 . . . Mexique. puberulus MannerH. Bull. Mosc. II,1843, p. 240 Californie. vittatus CAND. Monogr. II, p. 251 . . . . . Mexique. 
heteroderoides Sternh. Atti Soc. Ital. Sc. Nat.

XV, p. 567 . . . . . . . . Rép. Argentine. depressipennis CAvd. Monogr. II, p. 252 . Mexique. piceus Steinн. Col. Hefte XIV, p. 123 . . Nouv. Grenade. alternans Esch. Entomogr. 1822, p. 74 . . . Brésil. angustus CAND. Monogr. II, p. 252. . . . . Colombie. castanipes FABr. System. Eleuth. II, p. 234 . Guadeloupe. castaneus FABR. Ent. Syst.

substriatus CAND. Monogr. II, p. 25t. . . St-Domingue. pubescens CAST. Hist. Nat. I, p. 232 . . . . Brésil. partitus CAND. Monogr. II, p. 254 . : . . . j) sericeus CAND. Élat. nouv. I, p. 24. . . . . Guyane. pariphus CAND. Monogr. II, p. 255. . . . . Brésil. Gastelnaui CAND. Élat. nouv. II, p. 21 . . Bahia. truncatus CAND. Ibid. . . . . . . . Uruguay. murinus CAND. Ibid. IV, p.26 . . . . . . Brésil. posticus Eschs. Entomogr. p. 76 . . . . . » incultus CAND. Monogr. II, p. 256 . . . . . Paraguay. spurcus CAND. Ibid. p. 257. . . . . . . . lirésil. depressus Sol. Gax, Hist. Chile V, p. 17. . . Chili. aversus Lec. Trans. Am. Soc. X, p.482. . . Georgie. brunnipennis CAND. Monogr. II, p. 257 . . inuenos-Ayres. robustus HoRN, Trans. Soc. entom. Am., 1871, p. 311. . . . . . . . . . . . Arizona. sticturus CAND. Monogr. II, p. 260. . . . . Vénézuéla. lepidus Lec. Trans. Am. ph. Soc. X, 485. . . Georgie. sordidus Lec. Ibid. p.482 . . . . . . . . Rio-Gila. dubius CAND. Monogr. II, p. 260 . . . . C Cumana. athoides Lec. New. sp. Col. I, 1863, p. 84 . . Texas. tumidicollis CAND. Monogr. II, p. 26 . . . Guatémala. varians Steinh. Col. Hefte XIV, p. 121 . Nouv. Grenade. æoloïdes SternH. Ibid., p. 122 ocanianus Steinh. Mitt. Munch. Entomol. Ver. 1877, p. 80 . . . . . . . . . . Ocania. 
vespertinus FABR. Syst. El. II, p. 240. . . . Etats-Unis. finitimus $\mathrm{S}_{\mathrm{AY}}$, Trans. VI.

serotinus GERM. Zeitsch. I.

texanus CAND. Monogr. II, p. 262 . . . .

suturalis Lec. Trans. Am. Soc. 1853, p. 482.

Texas.

mutuus HorN, Am. ent. Soc. Tr. 1871, p. 312

Alabama.

peninsularis CAND. Élat. nouv. IV, p. 26 .

Arizona.

amazonicus CAND. Ibid. III, p. 53

Floride.

melanophthalmus CAND. Monogr. II, p. 264 .

Para.

serotinus $\mathrm{C}_{\mathrm{AND}}$. Élat. nouv. II, p. 21.

reductus CAND. Ibid.

Bahia.

decorus Burr. Stett. ent. Zeitschr., 1875 .Rép. Argentine. variegatus SternH. Col. Hefte XIV, p. 123. N. Grenade. auritus HerRst, Käf. X, p. 145 . . . . . . Etats-Unis.

crassicollis Melsh. Proc. Acad. Phil. II.

bellus SAY, Journ. Acad. Phil. III, p. 168 . . ”

finitimus CASEY, North. Am. Col. 1884, p. 170. New Jersey. blandulus Lec. Trans. Am. Phil. 1853, p. 483 . Georgie mér. pictus CAND. Monogr. II, p. 267 . . . . . Guatémala.

\section{Le Groupe.}

Australie de l'Est, du Nord et du Sud, ainsi quo la Tasmanie et la Nlle Zélande.

australasiæ BoIsd. Faun. Océan pac. 1835,

p. 34 . . . . . . . . Australie orient. basalis GyL. ScHONH. syn.?

erubescens CAND. Monogr. $\Pi$, p. 231 . . . . . is cerdo Erichs. Wiegm. Arch.1842, 1, p. 137 . . Adélaïde. tabidus ERICHs. Tbid. . . . . . . . . Melbourne.

crocopus Hope, Trans. Lond. 1845.

punciatostriatus CAND. Monogr. II, p. 236 . ”

aphiloïdes CAND. Tbid., p. 237 . . . . . . Sydney. Brucki CAND. Tbid., p. 236. . . . . . . . Melbourne. 
Gordieri Le Gurlu. Rev. Zool. 184t, p. 221 . Victoria. viduus CAND. Monogr. II, p. 243. cinereus Bu. Voy. Pôle Sud.

undatus CAND. Monogr. II, p. 229 . . . . N. Holl. sept. sulcatus CAND. Élat. nouv. II, p.18 . . . Q Queensland. fabrilis ErICHs. Wiegm. Arch. $18+2$ Tasmanie. fuscicornis ERICHs. Ibid. rutilicornis ERICHS. Ibid. socius CAND. Élat. nouv. II, p. 18 strigatus (pour striatus) CAND. Élat. nouv. II, brevipennis CAND. Monogr. II, p. 239. . . . Queensland. atratus MaC Leay, Tr. N. S. W. acuminatus MAC LEAx, Trans. N. S. Wales. II, ventralis CAND. Élat. nouv. II, p. 18 . . . Roctihampton. rectangulus CAND. Monogr. II, p. 233 . . . ” regularis CAND. Ann. Mus. Génov. XII, p. 115. Cap York. compactus CAND. Élat. nouv. II, p. 19. . . Rockhampton. striatus Mad Leax, Tr. N. S. W. Ent. Soc. II,

p. 252.

Queensland. rufifrons CAND. Élat. nouv. II, 18 . spatulatus CAND. Tbid. II, p.53 - . . . . Pydney.
seniculus CAND. Ibid. II, p.19 . . . . . Queensland. squalidus CAND. Ibid. II, p. 20 . . . . . Adélaide. macer CAND. Ibid. Melbourne. 
nebulosus Mac Leay. Trans. N.S. W.II, p. 254. Queensland. plagiatus CAND. Élat. nouv. III, p. 52. . . Rockhampton. nigripennis CAND. Ibid. II, p. 20 . . . . . Melbourne. planus CAND. Ibid., p. 19 . . . . . . . . Sidney. flavidus CAND. Ibid., p. 20. . . . . . . . ” flavicans CAND. Ann. Mus. civ. Genova XII, p. 116 simulans CAND. Tbid., p. 117 . . . . . ‘ . squalescens CAND. Notes of Leyd. Mus. IX,

p. 286. . . . . . . . . . . . . Carpentarie. basilaris CAND. Ann. Mus. Genova XII, p. 116. Cap York. Torresi CAND. Tbid. XV, p.190 . . . . . . Somerset. tumidus CAND. Notes Leyd. Mus. IX, p. 286 . Carpentarie. scapularis Guḱr. Voy. de la Coq., 1830, p. 69. Sidney. breviceps Mac Leay, Trans. N.S. W.II, p. 252. Queensland. Gandezei M. Leay, Ibid. 256. . . . . . . Gayndah. castaneipennis M. Leay, Tbid. . . . . . ” elongatulus M. LEAY, Ibid. . . . . . . ” fulvipennis M. LeAy, Ibid., p. 254 . . . . . ” Mastersi M. LeAY, Ibid., p. 252 . . . . . . ” minor M. LEAY, Tbid., p.253 . . . . . . . ” rubicundus M. LEAY, Ibid. . . . . . . . ” subflavus M. LEAY, Ibid, p. 255 . . . . . ” subgeminatus M. LEAY, Ibid. . . . . . ” submaculatus M. LEAX, Tbid. . . . . . ” sulcicollis Eschs. (') ThоN, Archiv. II, p. 31 . Australie. se Groupe.

Australie de l'Ouest.

Menevillei CAND. Monogr. II, p. 233 . . . . Swan-River. antennalis CAND. Élat. nouv. III, p. 52 . . Champion-Bay.

(') Je n'ai point vu ces treize dernières espèces, dont quelques-unes devront peutatre entrer en synonymie. 
planiusculus CAND. Élat. nouv. II, p. 19 . Champion-Bay. aurulentus CAND. Ibid., p.18. . . . . . . Swan-River. coxalis CAND. Monogr. II, p. 243 . . . . . ” carinatus CAND. Tbid., p. 238 . . . . . . » miser CAND. Tbid., p. 239 . . . . . . . ”

\section{Ge Groupe.}

Polynésie, Nlie Guinée et Iles voisines.

corniculatus CAND. Ann. Mus. Genova XII,

p. 113 .

Nouv. Guinée. pauperatus CAND. Tbid. . . . . . . . Ternate. horistonotus CAND. Ibid., p. 112 . . . Nouv. Guinée. contiguus CAND. Ibid., p. 114. . . . . . . ” cristatus CAND. Ibid. XV, p. 170 . . . . . ” umbraculatus CAND. Élat. nouv. I, p. 25 . . ” yulensis CAND. Ann. Mus. Genova XII, p. 112. I. Yule. arouensis CAND. Ibid. . . . . . . . . Arou. leucopoda Montr. Ann. Fr., 1860 . . . Nouv. Calédonie. variabilis Montr. Ins. Woodl. in Ann. Soc. Agr.

Lyon, 1857 . Ile Woodlark. ferrugineus Montrodz. Ann. Fr., 1860. tuberculatus Montr. Ins. Woodl. makirensis MonTr. Ibid. pallipes Eschs. Thon, Arch. II, 1, p. 31 . . . Polynésie. rufangulus Fatrm. Rev. Zool. exsul Sharp, Ann. a. Mag., 1877, p. 20. . . Nouv. Zélande.

\section{ge Groupe.}

Asie et Malaisie.

mogolus CANd. Monogr. II, p. 227 . . . Hindoustun sept. babylonicus CAND. Ibid. . . . . . . . Mésopotamie. capucinus CAND. (') Élat. nouv. III, p.51 . . Cochinchine.

(1) A peine différent du précédent. 
elegans CAND. Ibid. II, p. 17. . . . . . Himalaya. prionurus CAND. Monogr. II, p. 226 . . Hindoustan mér.,

Ceylan.

tenuis CAND. Tbid., p. 228 . . . . . . . . Bombay.

Dohrni CAND. Élat. nouv. II, p. 17 . . . . Himalaya.

monachus CAND. Ibid. III, p. 51 . . . . Bangkok.

longicollis CAND. Ibid. II, p. 17 . . . . . . Calcutta.

ferrugosus CAND. Ibid., p. 16 . . . . . . Ma!acca.

Candezei Krrsch, (') Mus. Dresd., 1875.

scutulatus CAND. Ann. Mus. Gênes, 1888, p.674. Birmanie.

vagus CAND. Ibid. . . . . . . . . . . »

centralis CAND. Ibid. . . . . . . . . . ”

ornatus CAND. Élat. nouv. IV, p. 27 . . . . Bengale.

fasciatus CAND. Tbid. I, p. 25. . . . . . Bornéo.

discoidalis CAND. Ibid. II, p.17. . . . . . Malaisie.

coangustatus CAND. Ibid. IV, p. 26 . . . Padang.

\section{ÆOLUS.}

Eschs. Thon, Arch., 1829, II, I, p. 33.

Caractères des Monocrepidius, mais ayant le quatrième article des tarses cordiforme, non lamellé. Généralement de couleur jaune, variée de noir. Presque tous américains.

\section{gection $\mathrm{E}$.}

Carène principale des angles postérieurs du prothorax atteignant l'extrémité de l'angle, qui est très aigu.

s. g. Pseudaeolus.

australis CAND. Monogr. II, p. 284. . . . .

Sidney. suillus CAND. Élat. nouv. II, p. 22 . . . . . ”

(') Nom déjà appliqué en 1873, par Nac Leay, à un Monocrepidius d'Australie.

Nota. Sous le nom de Honocrepidius, Motschulski a brièvernent fait connaitre les Élatérides suivants, de Ceylan. qui, je pense, ne doivent pas être compris dans le genre, tel qu'il est entendu ici. Ce sont peut-être des Heteroderes : II. angustitarsis, axillaris, flavipes, indicus, testaceus. Je n'en ai point vu les spécimens typiques. 
Waggæ CAND. Tbid. III, p. 54 . . . . . Riverina. versicolor CAND. Tbid. . . . . . Australie occid.

Eolus p. dits.

platynotus CAND. Monogr. II, p. 284.

Para.

bivittatus Krascr, Berl. ent. Zeitschr., 1873, p. 367.

Steinheili CAND. Élat. nouv. III, p. 54.

Pérou. livens CAND. Ibid., p. 55.

Bogota.

suturellus CAND. Elat. nouv. IV, p. 27

Para.

bicolor CAND. Ibid.

circumscriptus GerM. Ins. Sp. nov., p. 46.

》)

elegans CAND. Monogr. II, p. 285.

binotatus CAND. Monogr. $\Pi$, p. 287.

Antilles.

fuscatus Sternh. Col. Hefte XIV, p. 125 . Nouv. Grenade.

antennatus CAND. Monogr. II, p. 288.

Brésil.

selliger CAND. Ibid.

puerulus CAND. Ibid., p. 289

sexnotulatus CAND. Ibid., p. 290

bifasciatus CAND. Élat. nouv. I, p. 25.

quintus CAND. Monogr. II, p. 290

adustus CAND. Ibid., p. 291

sigillatus CAND. Tbid., p. 298.

signifer CAND. Ibid.

pulchellus CAND. Ibid., p. 293

trilineatus CAND. Ibid.

obliquus CAND. Tbid., p. 294

rubricatus CAND. Tbid.

Cuba.

rufulus CAND. Ibid., p. 295.

melliculus CAND. Ibid.

»

)

)

)

)

))

)

Mexique.

)

)

)

Texas.

Moreleti TARn. Col. Açores.

undulatus CAND. Ibid., p. 300.

Colombie.

amabilis Lec. Trans. Am. Phil. X., p. 485 . . Maryland.

scriptus FABr. Syst. Eleuth. II, p. 244 . . . Brésil. 
callizonus Gukr. Voy. Favor .

Pérou.

opacus CAND. Élat. nouv. IV, p. 27.

Para.

ruficeps CAND., Monogr. II, p. 298.

Minas.

Sahlbergi CAND. Ibid.

Brésil.

arcticollis CAND. Ibid., p. 299.

)

undulatus CAND. Ibid., p. 300.

Colombie.

scitus CAND. Tbid.

)

Garzoni Sternн. Col. Hefte XIV, p. 125. Nouv. Grenade. nigromaculatus Drapiez, Ann. Sc. Br. 1819, p. 271.

trifasciatus Stern. Col. Hefte XIV, p. 124.

depressus CAND. Monogr. II, p.301 - . . St-Domingue.

frivolus CAND. Ibid., p. 302 . . . . . . . 》

discicollis CAND. Tbid., p. 303. . . . . . Cuba.

exclamationis CAND. Ibid. . . . . . Carthagène.

cucullatus CAND. Tbid., p. 304 . . . . . . Vénézuéla.

anchoralis CAND. Ibid., p. 306 . . . . . . »

delectabilis CAND. Ibid., p. 305 . . . . Nouv. Grenade. melinostictus CAND. Ibid., p. 307 . . . . . Brésil.

epularis CAND. (Emend.). Élat. nouv. IV, p. 27. Amazones. pusillus CAND. Monogr. II, p.305 . . . . . Colombie. lepidus CAND. Ibid., p.307. . . . . . . B Brésil. ichnographicus CAND. Ibid., p. 308 . . . . » intricatus CAND. Ibid. . . . . . . . . . ” virgulatus CAND. Ibid., p. 309 . . . . . . ” lineatus CAND. Ibid., p. 310 . . . . . . . ” polygrammus CAND. Ibid., p. 311 . . . . . ” cinctus CAND. Ibid. . . . . . . . . . . » multisignatus Steiner. Munch. entom. Zeits.

1877, p. 82 . . . . Nouv. Grenade, Haut Amazones. fissus StervH. Tbid., p. 83 . . . . . Nouv. Grenade.

\section{Section 11.}

Carène des angles postérieurs du prothorax n'atteignant pas l'extrémité de l'angle, qui est prolongée, mais peu acuminée. Dernier 
segment de l'abdomen plus ou moins bombé et ponctué-rugueux, parfois granuleux.

Orpheus Caxd. Monogr. II, p.312 . . . Nouv. Grenade. designatus CAND. Ibid. . . . . . . Vénézuéla. Rodriguezi CAxd. Elat. nouv. IV, p. 28. . . . Guatemala. bisignatus Sternн. Mith. Munch. Entom. Ver.

1877, p. 82 .

Mannerheimi CAXD. Monogr. II, p. 313. nobilis CAND. Élat. nouv. II, p. 22 . retrofasciatus CAND. Monogr. II, p. 315 flavipennis CAND. Ibid. rubripennis CAND. Élat. nouv. IV, p. 29. Batesi CAND. Monogr. II, p. 316. melanurus CAND. Monogr. II, p. 316 . comis CAND. Ibid., p. 317 facetus CAND. Ibid., p. 318. Otti CAND. Tbid.

Saulcyi Guér. Voy. Favor., p. 30 . biplagiatus CAND. Monogr. II, p. 320. maculatus dE GEer. Mém. Ins., p. 163 angulatus FABR., CAND. Monogr. II, p. 321. achates CAND. Monogr. II, p. 322 concinnus CAND. Ibid. signatipennis CAND. Ibid. minarum Stenn. Col. Hefte XIV, p. 123. Nouv. Grenade. graphicus CAND. Monogr. II, p. 323 . . . . Brésil. elegantulus Buru. Stett. Ent. Zeitschrift, 1875, p. 268. pectoralis CAND. Élat. nouv. III, p. 55 amicus CAND. Monogr. II, p. 324 mundicollis CAND. Ibid., p. 325 . cribratus CAND. Élat. nouv. IV, p. 28. . . . unifasciatus FABR. System. Eleuth. II, 1. 245. similis Hebst, Käf., Cand. Monogr.
Nouv. Grenade. Brésil. )

Guyane. Brésil. Amazones. ))

Vénézuéla. Colombie. Guyane. Colombie. Pérou. Brésil. St-Domingue. Brésil. ) Guyane. Rép. Argentine. Vénézuéla. Brésil. ) ) Guyane. 
basalis SteTNH. Mith.Munch. Entom.Ver.1877,

p. 8. . . . . . . . . . . . . Nouv. Grenadè. quadrimaculatus Cand. Monogr. II, p. 326 . Vénézuéla. bicinctus Steinн. Col. Hefte XIV, p. 124 . Nouv. Grenade. posticus CAND. Monogr. II, p. 327 . . . . . ” cruciger SteinH. Col. Hefte XIV, 1877, p.124. ” quadriguttatus CAND. Monogr. II, p. 328. . Brésil. Mniszechi CAND. Élat. nouv. IV, p. 28 . . Nouv. Grenade. reniger CAND. Monogr. II, p. 328 . . . . . Brésil. cibaensis CAND. Ibid., p. 329. . . . . . St-Domingue. cruciatus CAND. Ibid. 330. . . . . . . . Brésil. verruculgsus CAND. Ibid. '. . . . . . St-Domingue. bisellatus CAND. Ibid., p. 331. . . . . . . Brésil. circumcinctus CAND. Ibid., p. 332. · . Nouv. Grenade. vittatus CAND. Ibid. .. . . . . . . . . Brésil. pictus CAND. Ibid., p. 333 . . . . . . . . Mexique. tœniatus CAND. Élat. nouv. II, p. 22 . . . . Guatémala. dorsiger Germ. Zeitschr. V, p. 167 (Ampedus). Minas. feretrum Sternн. Col. Hefte XIV, p. 126 . Nouv. Grenade. lateralis Steinн. Atti Soc. Ital. Sc. Nat. XV,

p. 567. . . . . . . . . . . . . . Répub. Argent. sexguttatus CAND. Monogr. II, p. 334 . . . Brésil. octoguttatus CAND. Ibid., p. 335 . . . . . ” æquinoctialis CAND. Ibid., p. 336. . . . Quito. corypheus CAND. Ibid. . . . . . . . . Brésil. macer CAND. Ibid., p. 337 . . . . . . . . ” brunneus CAND. Tbid., p. 338 . . . . . . . \% trachypygus CAND. Tbid. . . . . . . . : » variolatus CAND. Elat. nouv. II, p. 22 . . . Guyane. unicolor CAND. Ibid. III, p. 56 . . . . . . Amazones. gránulatus CAND. Monogr. II, p. 339. . . Nouv. Grenade. Leprieuri CAND. Ibid., p. 340. . . . . . . Guyane. stolatus CAND. Élat. nouv. I, p. 25 . . . . Vénézuéla. capnurus CAND. Monogr. II, p.340. . . . . Amazones. 
præstans CAND. Ibid., p. 341. . . . . . Colombie. apicalis CAND. Ibid. . . . . . . Amérique équat. proletarius Enichs. Schomb. Guyan. III, p.558. Guyane. nigrinus CAND. Monogr. II, p. 342 . . . . Nouv. Grenade. sanguinicollis Cand. Ibid., p. 343. . . Nour. Grenade. cruentus CAND. Ibid. . . . . . . . . » thoracicus CAND. Ibid., p. 344 . . . . . . »

\section{Heteroderes.}

Latr. Ann. Fr. 1834, p. 155.

Eolus pars. CAND. (').

Monocrépidiites à prothorax couvert d'un pointillé fin et serré, sur lequel se montre un pointillé plus gros et moins dense, en d'autres termes, ponctuation double.

Ier Groupe.

Espèces paléarctiques.

crucifer Rossi, Faun. Etr. I, p. 183 . . . Europe mér. atricapillus Germ. Ins. Sp. nov., p. 40 . . Russie mér. sarmaticus (Eolus) Мотsch. Mém. Ac. Pétersb.

1860, II, p. 516.

Rossii Germ. Zeitschr. V, p. 148 . . Cancase, Turkiestan. tesellatus Mотs. Bull. Acad. Pétersb. 1860.

cryptohypnoides CAND. Élat. nouv. III, p. 57. turcomanus CAND. Ibid. IV, p. 29 . . . . Turkestan. grisescens Germ. Zeitschr. V, p.151. . . Afrique sept., Russie mér., Asie mineure, Syrie. musculus Germ. Ibid. . . . . . . . Basse Egypte. modestus CAND. Monogr. II, p. 370 . . . . Algérie. algirinus Luc. Anim. Artic. de l'Algérie 1849, p. 166.

(') Les Aeolus, section III, de la Monographie, sont reportés dans les Heteroderes, qui suivent. 
ægyptiacus (') (Eolus) Mots. Bull. Acad. Pét.

1860, p. 518. . . . . . . . . Egypte.

2 croupe.

Espèces asiatiques, y compris la Nouvelle Guinée.

sequester CAND. Monogr.II, p. 378 . . . . Bombay.

lenis CAND. Ibid. I, p. 357 . . . . . . . . Bengale. sericeus CAND. Ibid. . . . . . . Hindoustan septent. macroderes CAND. Ibid., p. 358. . Hindoustan, Birmanie. ornatus ( $\left.{ }^{2}\right)$ CAND. Ann. Mus. Gênes, 1888 . . Birmanie. oblitus CAND. Monogr. II, p.361. . . . . . Ind. or. Iuteipes CAND. Élat. nouv. IV, p. 30 . . . . Bengale. negumbensis CAND. Monogr. II, p. 359 . . . Ceylan. spinicollis CAND. Ibid., p. 360 . . . . . . ” spinosus CAND. Bull. Soc. ent. belge 1890 . . Bengale. albicans CAND. Élat. nouv. $\Pi$, p. 23 . . . . Siam. malaisianus CAND. Monogr. II, p. 359 . . . Malaisie. cinerascens CAND. Ibid., p. $362 . \quad$. . . . . Népaul. triangularis EscHs. Entom. I, 1822, p. 73 . . Philippines, Malaise, Indes transg.

infuscatus Мотs. Bull. Ac. Sc. Pétersb. 1860, p. 518.

pardus CAND. Monogr. II, p. 345 . . . . . Ind. or. brachmana CAND. Ibid. . . . . . . . »

flavidulus Mots. loc. cit. chrysites CAND. Ibid., p. 346 . . . . . . Bombay. biellipticus Buyss. (Eolus) Ann. Fr. 1889, bull., p. 213. . . . . . . . . . . . Syrie. sinensis CANd. Monogr. II, p. 346 . . . . . Chine. agnatus CAND. Soc. Sc. Liége V, $2^{\circ}$ sér., p. $8 . \quad$ Japon.

(1) Cette espice, qui m'est inconnue, pourrait être l'H. musculus Germ.

(2) Je soupçonne l'Eolus nigrofasciatus Motsch. de lui être identique. 
multilineatus CAND. Ann. Mus. Gênes XII, p. 118.

Beccarii CAND. Ibid.

Célèbes.

minusculus CAND. Élat. nouv. II, p. 23 . I. Philippines. pusillus CAND. Élat. nouv. I, p. 28 . . . Nouv. Guinée.

\section{3e croupe.}

Espèces australiennes et polynésiennes.

albidus Mac. LeaY (Monocr.) Tr. N. S. W. II, p. 255.

Queenstand. instabilis Farrm. Rev. Zool. 1849, p. 35 . Taïti.

\section{areupe.}

Espèces africaines p. dites.

Waltli Cand. Monogr. II, p. 380. . . . . Haute Egypte. abyssinus CAND. Ibid., p. 366. Abyssinie. spissus Cand. Élat. nouv. IV, p. 29. ) crux CAND. Élat. nouv. III, p. 56. . . . . . ” juvencus CAND. Ibid. III, p. 57 . . . . . » mitigatus CAND. Monogr. II, p. 365 . . . . Afrique. kordofanus CAND. Ibid. II, p.364 . . . . . Kordofan. acutangulus Gerst. Beitr. Ins. Zanzib. 1871, p. 54 inops CAND. Monogr. II, p. 379 Zanzibar. fuscus Latr. Ann. Fr., 1834, p. 115. Sénégal. Guinée. murinus CAND. Monogr. II, p. 378 . ) inscriptus Erichs. Fn. Angol, 1843 . . . Afrique occid. plancus Erichs. (Monocrep) Ibid. . . . . . Angola. variolus CAND. Élat. nouv. IV, p. 29 . . . : Congo. cuneatus Qued. Deutsch. entom. Zeitschr., 1886 trifurcus CAND. Monogr. II, p. 348 Sénégal. zebratus CAND. Élat. nouv. IV, p. 30 
flavonotatus Bонем. Ins. Caffr. I, II, p. 407 patruelis CAND. Monogr. II, p. 363 . . . . tertius CAND. Tbid., p. 348 .

futilis CAND. Ibid., p. 379 æoloides CAND. Élat. nouv. III, p. 56 . . . trilineatus CAND. Monogr. II, p. 871 . . . pulchellus CAND. Élat. nouv. IV, p. 30 seniculus CAST. Hist. Nat. Ins. I, p. 246 coctus CAND. Monogr. II, p. 367. contractus Bонем. Ins. Caffr. I, II, p. 375 . effusus. Bонем. Tbid., p. 408 complanatus $\mathrm{K}_{L}$. Ins. Mad., p. 67 . rufulus CAND. Monogr. II, p. 365
Cafrerie. Sénégal.

))

)

Gabon.

Sénégal. Cap.

Sénégal. Cafrerie.

)) ) Madagascar. ))

\section{sic iroupe.}

Espèces américaines.

lantus CAND. Élat. nouv. IV, p. 31 . . . . Floride. heterostixus CAND. Ibid., p. 30 . . . . . Guatémala. Arechavaletæ CAND. Ibid. III, p. 58 . . . . Uruguay: rufangulus GrLlent. in ScнӧNн. Syn. Ins.

Append., p. 142. Brésil mér., République Argent., Uruguay, Paraguay.

asininus GERM. Zeitschr. I, p. 231. patagonus Stennh. Atti Soc. Hal. XI, p. 568. azoricus TARN. Col. des Açores, 1860, p. 93 . atlanticus CAND. Élat. nouv. I, p. 26.

caninus GERM. Zeitschr. I, p. 231 . . . . . Laurentii Guén. Voy. Favorite, 1838, p. 31 . attenuatus CAND. Monogr. II, p. 373 . Açores. amplicollis GYLL., loc. cit., p. 141 . . . . felinus CAND. Monogr. II, p. 376.

Brésil. Pérou. Bahia. Antilles. Colombie. bardus CAND. Ibid. .

$\stackrel{\text { Para. }}{\text { Par }}$ 


\section{TRIBU IX.}

\section{ÉLATÉRITES (').}

Front rebordé en avant; prosternum à sutures rectilignes ou concaves, simples; hanches postérieures dilatées en dedans; tarses simples, aucun article dilaté ou lamellé. Écusson non cordiforme.

\section{Drasterius.}

Eschs. Thon, Entom. Arch. II, 1, p. 33.

Caractères généraux des Monocrépidiites, mais à quatrième article des tarses ni dilaté, ni lamellé.

\section{I er Goupe.}

Ancien continent.

bimaculatus Rossi, Faun. Etrusc. I, p. 182 . Europe mér. Var. breviusculus (Agriotes) Dessr. Abeille, 1869.

flguratus Germ. Zeitschr. V, p. 148 . . . Europe orient. persicus (Ëolus) Motsch. Mém. Sc. Petersb., 1860.

prosternalis CAND. Élat. nouv. $\Pi$, p. 26. . . Himalaya. brahminus CAND. Monogr. II, p. 426 . . . » collaris CAND. Ibid., 427 . . . . . . . . Indes orient. sulcatulus CAND. Ibid. . . . . . . . . ” indus CAND. Tbid., p. 428 . . . . . . . . Bombay. apicalis CAnd. Ann. Mus. Gênes XII, p.120 . Célèbes. insularis CAND. Bull. Soc. ent. Belg., XVIII,

p. 121. . . . . . . . . . Philippines. cambodiensis FleUt. Ann. Fr., 1889, p. 141. Indo-Chine. illinitus CAND. Ellat. nouv. IV, p. 33 . . . . Boméo. capensis CAND. Ibid. . . . . . . . . Cap. æthiopicus CAND. Ibid. III, p.62 . . . . Abyssinie.

(1) Cette tribu occupe la place de celle des Physorhinites qui, dans la Monographie, vient apres les Honocrépidiites. Cette transposition a pour motif l'affinité étroite qu'ont les Drasterius, premier genre des Élatérites, avec les Hcteroderes qui précèdent. 
brevipennis CAND. Ibid. . . . . . . . Abyssinie.

umbrosus Erichs. Wiegm. Arch., 1843 . . . Angola.

\section{$\rightarrow$ Crompe.}

Nouveau continent.

elegans Fabr. Ent. System. I, II, p. 230 . Amér. bor. mérid. dorsalis (SAY) CAND. Monogr. (Eolus). comis Lec. Trans. Am. Ph. Soc. X. marginicollis HoRN, Ibid. 1871. variegatus Bонем. Eugen. Res., 1858.

livens Lec. Trans. Am. Phil. Soc. X, p. 484. . Californie. asper Lec. Trans. Am. entom. Soc, 1884 . . Texas. cribratus LEC. Ibid. . . . . . . . . . ” debilis Lec. Ibid. . . . . . . . . . . Lac Supér. grandicollis HonN, Ibid., 1871, p. 308. . . . Californie. fretus CASEY, North Amer. Coleopt., 1885,

p. 171. . . . . . . . . . . . . Philadelphie. simiolus CANd. Monogr. II, p.428 . . . . Mexique. Dugesi CAND. Élat. nouv. IV, p. 33.

Gaudichaudi Gúkr. Voy. de la Favor., 1838, p. 32 pullatus Cand. Monogr. II, p. 432 . . . . Buenos-Ayres. pictus Sternh. Soc. Ital. Sc. Nat. XV, p. 568 . ”

\section{Grammophorus.}

Sor. GAY, Hist. Chil. V, 1851, p. 20.

Caractères des Drasterius, sauf les tarses munis de brosses en dessous; insectes propres au Chili.

rufipennis Sor. GAY, Hist. Chil. V, p. 25 . .

Chili. Solieri CAND. Monogr. II, p. 419. … . . ” impressicollis SoL., loc. cit. . . . . . . . ” niger SoL. Ibid. 


\section{Elastros.}

CAND. Monogr. II, 1859, p. 433.

Sutures prosternales concaves et creusées on avant; premier article des tarses long.

sardioderus CAND. Monogr. II, p. 435 . . . Madagascar. anchastinus CAND. Tbid. . . . . . . . » tepidus CAND. Ibid. ampedioides FATRM. Ann. Fr., 1871, p. 38 . . ” submurinus FaIRM. Ibid. . . . . . . . ” senegalensis CAND. Élat. nouv. III . . . . Sénégal. dolosus Crotch, Proc. Zool. Soc., 1867, p. 386 . Arores. flavipes MAC LEAY ('), Tr. N. S. W. II, p. 257 . Queensland.

Elater.

LnNNE, System. Nat., 1767.

Front bombé, rebordé en avant; écusson non cordiforme; sutures prosternales concaves et creusées en avant; hanches postérieures dilatées assez brusquement dans leur portion interne; tarses filiformes, à articles diminuant graduellement de longueur jusqu'au $4 \theta$ inclus; ongles simples.

\section{Ier Groupe.}

Ancien continent.

A. Bicolores ou tachetés.

sanguineus Linné, Syst. Nat. I, II, p. 654. Europe, Asie sept. haemelyter ScHr. Faun. Boic., p. 590.

rufipennis, seminuber Sтерн. Illustr. Brit. III, p. 256, 257.

cinnabarinus Eschs. Tнол Arch. II, p. 1, 33 . Europe sept. satrapa Kresenw. Naturg. Ins. Deutschl.

angusticollis V. HEyD. Deuts. ent. Zeitschrift, 1886, p. 278. . . . . . . . . . Asie mineure.

(1) Je doute que cette espèce appartienne au genre. 
sanguinolentus Schrank, Ent. Beitr. I, p. 69.

Europe.

ephippium OL. Ent.

haemopterus LnvNí, Gmelin. ed.

sanguineus var. PAYK. ILLIG.

Pomonæ Steph. Illustr. Brit. III, p. 257.

Europe.

Var. Gerardi Cand. Bull. Soc. Belg., 1890.

præustus FABR. Entom. System. I, II, p. 229.

)

coccinatus Rye, Monthl. Mag., 1867.

sanguineus var. $b$. PAYK.

cardinalis Schіӧdте, Kr. Natur. 1865.

aurilegulus ScHAdF. Isis, 1862.

ferrugatus LAC. Faun. Par. p. 655.

)

crocatus Steph. Man. Brit.

elongatulus Zetrerst. Ins. Lapp.

pomorum Herbst, CAND. Mon.

rubidus CAND. Monogr. $\Pi, 447$. . . . . Allem. mérid.

erubescens Eschs. ThоN Archiv. II, p. 133. . Enrope. crocatus CAST. Hist. Nat., CAND. Monogr.

ochropterus Eschs. Die Quatemb., 1830 . Europe orient. var. straminipennis HEYD. Zeitsch. Berl., 1880,

p. 289 . . . . . . . . . . . . Asturies.

cænobita Costa, Voy. en Calabr., 1881 . . . Calabre.

ochripennis ReIT. Wien Entom., 1881.

rufiventris CAND. Monogr. II, p. 450. . . . Espagne.

elongatulus FABR. Mantiss. I, p. 144 . . . . E Europe.

gliscereus Pz. Entom. Tasch. p. 241.

proustus STEPH. Man. Brit.

balteatus Linné, Syst. Nat. I, II, p. 654 . . .

)

pulcher BAUdI, Berl. Ent. Zeits., 1871, p. 50.

Chypre.

elegantulus ScнöNн. Syn. Ins., p. 1, 3, p. $303 . \quad$ Europe.

adustus Esон. Thом Arch.

austriacus CAST. Hist. nat.

elongatulus HerBst, Käf.

puniceus Lewis, Entom. Monthl. Mag. 1879 .

Japon. 
rubridorsus Lewis, Entom. Monthl. 1879 . . Japon. azurescens CAND. Élat. nouv. I, p. 29 . . . quadrisignatus GYLL. Sснӧхн. Synonym. Ins.

App., p. 139

Allemagne. Chalusi GuÉr. Rev. Zool. 1847, p. 7.

tristis Lrvvé, Syst. Nat., p. 1, 2, 655. . Europe, Asie sept. basalis Mannere. Bull. de Mosc., 1852.

sinuatus GERr. Zeitschr. V, p.170 . . . . . Hongrie. erythrogonus Mulu. Germ. Mag. IV, p.186 . Europe. ruficeps Muxs. Op. Ent. IV, p. 29. . . Frunce, Allem. mér. pallidus Redt. Faun. Austr. 2॰ éd., p. 500. cuneiformis HaMre, Berl. Zeitschr. 1866. conspurcatus CAND. Ėlat. nouv. IV, p. 34 . . Philippines. melanurus Muls. Ann. Soc. Lin. Lyon 1855 . France or. circassicus Reitter, Wien Ent. Zool.VI,1887. Caucase. auranticulus REIT. Ibid. . . . . . . . » ochrinulus ReIT. Ibid. . . . . . . . . Crimée.

B. Unicolores, généralement noirs.

Megerlei Lac. Faun. Par. p. 656 Europe. var. melanotites GREDL. Käf. Tyr. 1863.

ætbiops Lac. Faun. Par. p. 657 .

Europe. brunnicornis pars. GERM. Zeitschr. V. scrofa GERM. Ibid. concolor Strerlin, Mittheil, Schw. Ges. I, 1862, p. 36 nigerrimus LAC. Faun. Par. p. 657.

Suisse. brunnicornis pars. GERM. loc. cit. V. obsidianus GERM. Ibid. fuscicornis CAND. Monogr. I, p. 475 . . . . nigrinus Herbst, Fuesl. Arch. V, p. 114. . Indes or. anthracinus LEC. (Am. bor.). pilosulus Herвst, Käf. rufitarsis DESTIGNES, Entomol. 
compactus CAND. Emend. sequentis . . . . Amur.

Candezei Kraatz, D. Entom. Zeitschr. 1879, p. 123.

picitarsis Mots. Schr. Reis. 1860, p. 110 . . . ” simillimus $\mathrm{Kr}$. loc. cit.

pallipes KraAtz, loc. cit. . . . . . . . . . ”

sobrinus Mотsсн. Ibid., p. 111 . . . . . . »

dilutipes Мотsсн. Ibid. . . . . . . . . »

hypogastricus Cand. Mém. Soc. Liége V, $2^{\mathrm{e}}$ sér. p. 9 . . . . . . . . . . Japon.

bicarinatus CAND. Ibid. . . . . . . . . ”

Ryei Lewis, Monthl. Mag. Décemb.1879. . . ”

carbunculus LewIs, Ibid. ...... ”

fulvago Mars. Abeille V, 1868, p. 176. . . . Syrie.

lepidus MäkuIN, Ofv.Finsk. Vet. Soc. 19,p. 30 . Sibérie.

subcostatus Kolbe, Arch. f. Nat. 1887, p.197. Corée.

analogicus KolBe, Ibid., p. 198. . . . . . . ”

\section{tu Groupe.}

Nouveau continent.

hepaticus Mels. Proc. Acad. II, p. 160 . . Pensylvanie. insignis Lec. An. ent. Soc. Tr. 1884 . . . . Texas. rhodopus Lec. Pacif. R. R. Expl. XI, p. 47. . Orégon. umbricolor МотsсH.

rubriventris Lec. Tbid. . . . . . . . . N. Mexique. partitus CAND. Élat. nouv. III, p. 63 . . . . Californie. fastus Lec. Am. entom. Soc. Tr. 1884 . . . ” melinus LEc. Ibid. . . . . . . . . . . ” Phelpsi HorN, Am. ent. Tr. 1874, p. 22 . . . ” Horni CAND. Élat. nouv. III, p. 63 . . . . . ” cordatus HoRN, loc. cit., 1871, p. 306 . . . . ” Behrensi Hors, Ibid., p. 214 . . . . . . . ” cordifer LEC. Proc. Acad. 1859, p. 72 . : : : 》

Lecontei CAND. Monogr. II, p. 459. 
ater LEc. Tr. Am. ent. Soc. 1884

Colorado.

carbonicolor Eschs. ThоN Arch. II, p. 33 . . Sithlia. pedalis GerM. Zeitsch. V, p. 176. . . . . . Etats-Unis. ursulus Messh. Pr. Acad. II.

deletus Lec. Tr. am. ph. Soc. X.

mufipes BeAuv. Ins., p. 214?

manipularis Cand. Monogr. II, p. 478 . . Pensylvanie. longicornis Lec. Tr. Am. ent. Soc. 188t . . Californie. mixtus Herbst, Käf. X, p. $7 t$. . . Etats-Unis du Nord. lacustris, fusculus, miniipennis, Lec. loc. cit.

pullus Gerr. Zeitschr. V, p. 162. . . . . ”

nigricollis Herbst, Käf X, p.73 . . . . ”

linteus SAY, Trans. Am. Soc. VI, p. 168 . . . ”

lugubris GERM. Zeitschr. V.

discoideus FABr. System. Eleuth., p. 240 . . ”

vitiosus Lec. Tr. Am. phil. Soc. X, p. 465 . . Vermont.

læsus Lec. Tbid. . . . . . . . . . . . Georgie.

Sayi Lec. Tr. Am. phil. Soc. X, p.465. . . . Etats-Unis. oblessus SAY, Tbid. VI.

impolitus Mersh. Proc. Acad. I, p. 160. . . ”

socer Lec. Tr. Am. phil. Soc. X, p. 467 . . . Pensylvanie.

rubricollis Herist, Käf. X, p. 49 . . . . . Etats-Unis.

verticinus BEAUV. Ins., p. 214.

semicinctus RAND. Bost. Journ. II, p. 10 . . ” protervus Lec., loc. cit.

militaris HarRIs, Hartf. Trans., 1836, p. 70 subtilis Lec. Tr. Am. entom. Soc., 1884 . . . Lac Supér. luctuosus Lec. Tr. Am. phil. Soc. X, p.466. . Californie. nigricans GeRM. Zeitschr. V, p. 176 . . . . Etats-Unis. testaceipes Mrish. Proc. Ac. II, p. 243. molestus LEc., loc. cit., p. 467.

rubricus SAy, Ann. Lyc. I, p. 261 . . . . Caroline N. concinnus GERM. Zeitschr. V, p. 170.

lateralis Lec. Tr. Am. entom. Soc., 188t . . Nevada. 
atripennis Hons, Ibid.1871, p. 306 . . . Californie. violaceipennis CAND. Élat. nouv. III, p. 62.

collaris SAy, Ann. Lyc. I, p. 268 . . . . . Etats-Unis. thoracicus Henbst, Käf. X, p. 51.

torquatus Lec. Tr. Am. entom. Soc. 1884 . . Nevada. nevadensis Lec. Ibid. .

sanguinipennis SAY, Journ. Acad. III, p.177. États-Unis. xanthomus Germ. Zeitschr. V, p.168 . . . Pensylvanie. humeralis MeLsh. Proc. Acad. II.

dimidiatus Lec. Proc. Acad. 1861, p. 347 . . Californie. apicatus SAY, Trans. Am. phil. Soc. VI, p. 170. États-Unis. melanopygus GERM. Zeitschr. V.

phonicopterus Germ. Tbid., Cand., Lec.

affinis LEc. Tr. Am. entom. Soc., 1884.

Californie. obliquus SAY, Trans. Am. phil. Soc. VI., p.174. Etats-Unis. areolatus SAY, Journ. Ac. Phil. ПI, Lec., CAND.

scitulus Germ. Zeitschr. V, p. 168.

pusio Germ., loc. cit., p. 169

luteolus Lec., loc. cit, p. 471.

ignobilis Bонем. Eug. Res., p.68 . . . . . Californie. arctus CAND. Monogr. II, p. 435. . . . . . Vera Cruz.

3 eroupe.

Australie et Polynésie.

Guillebeaui Perroud, Ann. Soc. Lyon., 1864,

p. 112. . . . . . . . . . . Nouv. Calédonie.

Boisduvali Fauv. (sub.nom Ludius) Bull. Soc.

Linn. Norm., 1867, p. 185. . . . . . . . »

Gandezei Fauv. Ibid. . . . . . . . . . ”

asperulus CAND. Élat. nouv. III, p. 65 . . . Australie.

australis CAND. Monogr. II, p. 483 . . . . . »

perplexus CAND. Ibid., p. 482. . . . . . . Melbourne.

pusillus CAND. Élat. nouv. II, p. 26. . . . . Adélaïde.

Mastersi M. LeAY, Tr. N. South. W. II, p. 257. Queensland. 


\section{ANTITYYPUS.}

CAND.

Elatei FATRM.

Hanches postérieures peu élargies en dedans; facies des Elater.

insignitus FAIRMr. Rev. Zool., 1860, p. 268 . .

Chiti.

\section{Parelater.}

CAND.

\section{Elater CAND.}

Ne diffère des vrais Elater que par sa forme plus parallèle, ses élytres fortement sillonnées qui lui donnent un facies différent.

coccineus CAND. Élat. nouv. I, p. 29 . . . . Indes orient.

\section{HOMOTECHNES.}

CAND. Élat. nouv. III, p. 71.

Mésosternum horizontal; facies de Corymbites.

corymbitoides CAND., loc. cit. . . . . Chine centrale.

\section{Bladta.}

Lec. Tr. Am. Phil. Soc. X, p. 472.

Ampedus Germ.

Caractères des Elater avec les tarses courts, leur premier article égal aux deux suivants réunis, les articles $2-4$, spongieux en dessous, leur bord antéro-inférieur brièvement lamellé aux antérieurs seulement.

cribraria Gerur. Zeitschr. V, p. 178 . Etats-Unis, Georgie. cauta LEC. loc. cit.

\section{Elatrinus.}

Horn, Am. ent. Soc. 1871, p. 307.

Comme Elastrus, mais à 30 article des antennes aussi long que les suivants.

anthrax HoRn, loc. cit. . . . . . . Californie. 


\section{EOPENTHES.}

Sharp, Trans. Ac. roy. Dublin 1883, p. 153

Forme intermédiaire entre les Elater et les Megapenthes; le fron $t$ court et arrondi en avant; cavité mésosternale à bords perpendiculaires en avant.

basalis SHARP, loc. cit., p. 152 . . . . . . 1. Havä̈.

obscurus Sharp, Ibid. . . . . . . . . . »

debilis Sharp, Ibid., p. 154. . . . . . . . ”

satelles SHARP, Ibid. . . . . , . . . . ”

konæ SHARP, Ibid., p. 155 . . . . . . . . ”

ambiguus SHARP, Ibid. . . . . . . . . ”

humeralis (Elater) Karsch, Berl. Ent. Zeits.

1881 . . . . . . . . . . . . »

\section{IsCHNODES.}

Eschsch. Silb. Rev. IV, 1836.

Megapenthes CAND. Mon.

Forme plus large que les Megapenthes qui suivent.

sanguinicollis Panz. Fn. Germ. VI, p.13 . E Europe.

fulvicollis STEPH. ruficollis Donov.

Reedi CAND. Élat. nouv. III, p.64 . . . . . Chili.

\section{Megapenthes.}

Krasenw. Nat. Ins. Deutsch. IV, p. 353.

Elater, Ludius, LEc. 1884. Aphanobius, GerM.

Sutures prosternales fines, rectilignes; antennes linéaires, de longueur ordinaire.

\section{Ier Groupe.}

Ancien continent.

tibialis Lac. Faun. Par. 1835, p. 688 . . . . Europe.

cothurnatus Curris, Trans. Ent. Soc. Lond. 1854.

subcarinatus GERM. Zeitschr. V.

divaricatus DesBr., 1873. 
Volxemi CAND. Élat. nouv. II, p. 26

Caucase.

var. fulvescens Reitter, Wien Ent. Z., 1887.

lugens W. REDT. Dissert., 1842, II .

Europe. aterrimus Curtis, Brit. ent., p. 694.

gagates Curtis, Trans. ent. Soc., 1854.

carinifrons DesBr. (Agriotes) Opusc. entom. 1874, p. 40 .

Caucase. funebris CANd. Élat. nouv. III, p.6t . . . Chine centr. rutilipennis CAND. Monogr. II, p. 502 Perse. biplagiatus CAND. Élat. nouv. II, p. 37 flavescens CAND. Ibid.

Goquereli FAIrur. Ann. Soc. ent. Fr.,1871, p.41. modestus CAND. Monogr. II, p. 507 Hindoustan. mæstus CAND. Bull. Soc. entom. Belg., 1890 Bengale. linearis CAND. Élat. nouv. I, p. 30 . . . . Dargeeling. corrosus CAND. Ibid. . . . . . . . . . Bengale. agrotus CAND. Ibid. . . . . . . . Cambodge. piceus Flect. Ann. Fr. 1889, p. 142 . . . Cochinchine. bilæsus CAxd. MIonogr. II, p. 505 . . . . . Boméo. melanopterus CANd. Ibid., p. 502 . . . . Mysore. luteipes Hope, Tr. ent. Soc. 1845, p. 9. . . . Chine. vicinus Fledt. Ann. Fr. 1889, p. 142. . . . Cochinchine. tetricus CAND. Monogr. II, p. 504 . . . . . » mutulus CAND. Ibid., p. 506 . . . . . . Java. ebriolus CAND. Ibid. . . . . . . . . ” emarginatus CAND. Ibid., p. 507 . . . . ” bifasciatus CAND. Ellat. nouv. I (Melanox.). . Malacca. infumatus CAND. Ann. Mus. Gênes XV, p. 194. Sumatra. epitrotus CAND. Élat. nouv. I p. 32. (Melanox). Singapore. pauper CAND. Élat. nouv. II, p. 26. . . . . Inde transg. thoracicus Ktrsch, Mith. Mus. Dresd., 1875, p. 8. sondanicus CAND. Élat. nouv. III, p. 66 . Malacca. cinereus CAND. Ibid. IV, p. 35 Malaisie. Java. 
ligatus Cand. Ann. Mus. Gênes XII (Melanox). Hong-Kong. hirtus CAND. Élat. nouv. II, p. 27 . . . . . Birmanie. vittatus CAND. Ibid. I, p. 32 (Melanox.). . . Bornéo. basalis CAND. Ann. Mus. Gênes XV, p. 193 . Sumatra. birmanicus CAND. Ann. Mus. Gênes; 1888, p. 677.

moderatus CAND. Ibid., p. 678

lugubris CAND. Ibid.

Birmanie.

rufus CAND. Monogr. II, p. 503

)

))

inconditus CAND. Ibid., p. 504

Hindoustan.

Philippines.

opacipennis CAND. Ann. Soc. Belg., 1875., Bull. junceus CAND. Tbid.

inflatus CAND. Ibid.

angulosus $\mathrm{C}_{\mathrm{AND}}$. Ibid.

Mindanao.

diploconoides CAND. Ibid.

Philippines.

nigricornis CAND. Ibid.

niger CAND. Ann. Mus. Gênes XII, p. 121

Saleyeri CAND. Sequentis emendatio .

Célèbes.

Saleyer.

agriotides CAND. Notes Leyd. Mus. XV, p. 12. inficetus CAND. Ibid., p. 210 .

Sumatra.

anceps $C_{A N D}$. Ibid., p. 211

curtus CAND. Élat. nouv. II, p. 27.

)

Siam.

dorsalis Cano. Ann. Mus. Gênes XII, p. 123 .

Malaisie.

longus CAND. Élat. nouv. III, p. 65.

Siam.

rugipennis CAND. Ibid. IV, p. 34

Sumatra.

secundus CAND. Ibid.

Bornéo.

carinatus CAND. Ann. Mus. Gênes XII, p.123.

marginatus CAND. Ibid., p. 120.

)

》

opacus CAND. Mém. Soc. Sc. Liége, V, $2^{n}$ série.

gracilis CAND. Tbid.

Japon.

octoguttatus CAND. Élat. nouv. III, p. 66 . . Chine centr.

fusconiger Kolbe, Arch. f. Nat., 1888, p. 199.

Corée. 
Groupr.

Nouveau continent.

turbulentus Lec. Trans. Am. Ph. Soc. X, p. 463 .

Californie. aterrimus Hors, Trans. Am. entom. Soc., 1871,

p. 309.

granulosus MeLsh. Proc. Acad. nat. Sc. II

Sturmi LEC., loc. cit.

Sturmi Germ. Zeitschr. $\nabla$, p. 188 .

Cuba. nigriventris LEc. Trans. am. entom. Soc. 1884. Califormie. rufilabris GERM. Ins. Sp. nov., p. 47 . Etats-Unis. elegans Hons, Tr. am. ent. Soc. 1871, p. 310 . Culifornie. stigmosus Lec. Tr. am. phil. Soc. X, p. 472. Etats-Unis N. caprella LEC. Pac. Expl.

quadrimaculatus HorN (sub nom. Limonius), loc. cit., 1871, p. 313.

Rogersi HonN, Ibid.

lepidus Lec, Tr. am. ent. Soc. 1884 opaculus CAND. Élat. nouv. I, p. 29

Californic. tœniatus CAND. Tbid, p. 30

Cuba.

athoides CAND. Monogr. II, p. 499. )) psittaculus CAND. Ibid., p. 498.

Mexique. brasilianus CAND. Élat. nouv. II, p. 66 . Vénézuéla. flavonotatus CAND. Ibid. IV, p. 35.

Brésil.

3 Groupe.

Australie, Nlle Guinée, NHle Zélande.

automolus CAND. Monogr. II, p. 495 . . . Australie occ. dolens CAND. Tbid. . . . . . . . . ” apicatus CANd. Élat. nouv. I, 1865, p. 31. . . ” rufipes CAND. Ibid. . . . . . . . . . Adélaïde. lituratus CAND. Notes Leyd. Mus. IX, 1887. Carpentaria B. futilis CAND. Tbid. suturalis CANd. Ann. MIus. Gén. XII, p. 122. Nouv. Guinée. 
punctatus Cand Ibid. . . . . . . Nouv. Guinée.

agriotides CAND. Élat. nouv. I, p. 31 . . . . ”

lateristrigatus Wніте, Voy. Ereb. a. Terr. Nouv.Zélande.

\section{Melanoxanthus.}

Eschs. Silb. Rev. IV, 1836.

Mêmes caractères que les Megapenthes; antennes généralement plus courtes, dentées en scie, souvent épaissies de la base au sommet; presque tous de couleurs variées; propres à l'ancien continent.

melanocephalus FABR. Spec. Ins. I, p. 272. Régions tropic. universelles.

dimidiatipennis CAND. Monogr. II, p. 513. . Bengale. senegalensis CAND. Ibid., p. 514 . . . . . Sénégal. abyssinus CAND. Élat. nouv. I, p. 36 . . . . Abyssinie. inæqualis CAND. Ibid. III, p.69. . . . . . Gabon. melanurus CAND. Ibid. II, p. 27. . . . . . Siam. biplagiatus CAND. Monogr. II, p. 514. . . . Guinée. bilunatus CAND. Élat. nouv. I, p.36 . . . Gabon. lateplagiatus FAIRM. Ann. Fr. 1871, p. 41. . Madagascar. nigriventris CAND. Élat. nouv. IV, p. $36 \ldots \ldots$. . binus CAND. Ibid. . . . . . . . . . . » variolosus CAND. Ibid. II, p. 28, . . . . . Birmanie. rubiginosus CAND. Ibid., p. 29 . . . . . . Darjeeling. dorsatus CAND. Soc. entom. Belg. Bull. 1890 . Bengale. carbunculus CAND. Ibid. . . . . . . . . " " cobrella CAND. Élat. nouv. I, p. 34. . . . Hindoustan. rufotactus CAND. Ibid. II, p. 28. . . . . . Siam. fractus CAND. Ibid. . . . . . . . . . . Penang. decimus CAND. Ibid. . . . . . . . . . » flavidus CAND. Ibid., p. 29. . . . . . . . Bintang. quadrinotatus CAND. Ibid. I, p. $35 \ldots$... Ceylan. undatus CAND. Monogr. II, p. 518 . . . . . . . 
variegatus CAND. Ibid., p. 516

Ceylan.

vitticollis CAND. Ibid., p. 517.

)

scitulus CAND. Ibid., p. 518

inflexus CAND. Élat. nouv. I, p. 34

litura CAND. Ibid., p. 33.

dolosus CAND. Ibid.

physorhinulus CAND. Ibid.

signatus CAND. Tbid., p. 34

albofasciatus $\mathrm{C}_{\mathrm{AND}}$. Ibid., p. 35

promecus CAND. Ibid., p. 36

I. Philippines.

exclamationis CAND. Bull. Soc. Belg. XVIII,

1875, p. 123.

terminatus CAND. Ibid.

approximatus $\mathrm{CAND}_{\mathrm{AND}}$. Ibid.

rhomboidalis CAND. Mbid.

sextus CAND. Ibid.

decemguttatus CAND. Ibid.

bipartitus CAND. Ibid.

infimus CAND. Tbid.

amplicollis CAND. Élat. nouv. IV, p. 36 .

comosus CAND. Élat nouv. IV, p. 36

)

)

)

)

)

Mindanao.

))

)

Andamans.

Bornéo.

cuneatus CAND. Ibid. I, p. 33.

guttulatus CAND. Ibid.

quadrillum CAND. Ibid.

flavangulus CAND. Ibid.

bivittatus CAND. Ann. Mus. Gênes XII, p.128.

Doriæ CAND. Tbid.

ligatus CAND. Ibid.

brunneus CAND. Toid.

nigricollis CAND. Ibid.

decemmaculatus CAND. Notes Leyd. Mus. V,

p. 211.

cylindricus CAND. Ann. Mus. Gênes XV,

p. 194 
Lansbergei CAND. Élat. nouv. III, p. 67. . . Sumatra. granum CAND. Notes Leyd. Mus. IX, p. 191 confusus CAND. Ibid. II, 1880.

)

)

Java.

zebra Wredem. Zool. Magaz. II, I, p. 107

)

)

)

)

)

)

)

Célèbes. ducalis CAND. Ibid., p. 67

filiformis CAND. Tbid., p. 68

pusillus CAND. Élat. nouv. I, p. 36 .

rufinus CAND. Ibid., p. 35 .

tæniatus CANd. Ann. Mus. Gênes XII, p. 129.

morio CaND. Ibid.

subcylindricus CAND. Élat. nouv. I, p. 35.

partitus CAND. Ann. Mus. Gênes XII, p. 125.

angularis CAND. Tbid., p. 129.

Nouv. Guinée.

ruficollis CAND. Tbid., p. 127 .

)

tetraspilotus FAIRM. Le Naturaliste, mars

1883

Nouv. Bretagne.

semitinctus Вонем. Res. Eugen. p. 71 . . . I. Keelings.

cuneiformis CAND. Élat. nouv. III, p. 69. . . Sydney.

dimidiatus CAND. Élat. nouv. $\Pi$, p. 29 . . . ग

ferrugineus CAND. (Emend.) Ibid. au lieu de: Australie.

brunneus $\mathrm{C}_{\mathrm{AND}}$. Ibid.

australis CANDD. Monogr. II, p. 520.

)

dilaticollis CAND. Élat. nouv. III, p.69 . . Queensland.

Note. Motschulski a fait connaitre, en 1859 , les espèces suivantes de Ceylan; que je nai point vues et dont les noms font probablement double emploi, pour la plupart : M. bivillis, flovopictus, flavoscriptus, nigropunctotus, pictus, submaculatus, tessellatus, vitticollis, xanthographus. C'est aussi à ce genre qu'il faut sans doute rapporter les Agriotes Australasice (Arou) et quadripunctatus /Australie) de Blanch., voy. au Pôle Sud IV, p. 90. 


\section{AaLOPHUS (1).}

Sharp, Annals and Mag. 1877, p. 15.

Hanches postérieures très réduites; sutures prosternales à peine indiquées, sinueuses en avant.

modestus Sharp., loc. cit. . . . . Nouv. Zélande.

Lomenus.

Sharp, Ann. and Mag., 1877, p. 16.

Peu différent du genre précédent; les sutures prosternales plus droites.

pilicornis Sharp, loc. cit., p. 15. . . Nouv. Zélande. pictus Sharp, Ibid. . . . . . . . . . ” suffusus SHARP, Ibid. . . . . . . . . ” flavipes Sharp, Tbid. . . . . . . . . . ” similis SHarp, Tbid. . . . . . . » obscuripes SHaRP, Ibid. . . . . . . . . ” elegans Sharp, Ibid. . . . . . . . . . ” collaris Sharp, Ibid. . . . . . . . . ”

OXYsTHETUS.

FAIRm. Le Naturaliste, mars 1853.

Mésosternum réuni au métasternum, sans suture distincte. scapulatus Fatrm., loc. cit. . . . Nouv. Bretagne.

YPSILOSTHETUS.

CANd. Monogr. ПI, p. 521.

Mésosternum horizontal; tarses à crochets dentés. semiotulus CAND., loc. cit., p. 522 . . . . Nouv. Grenade.

(1) Comme pour beaucoup d'Llatérides de la Nlle Zélande, la place de l'espèce qui forme ce genre est difficile à déterminer. Elle a des affinités avec les Cryptohypnites, une apparence d'Anchustus, toutefois avec les caractrres négatifs des Elatérites qui s'opposent àt son introduction dans une autre fribu que celle-ci. 


\section{DODECACTENUS.}

CAND. Élat. nouv. IV, p. 36.

Mésosternum horizontal, non séparé du métasternum par une suture; crochets des tarses pectinés.

Staudingeri CAND., loc. cit. . . . . . . . Véragua.

\section{TRIBU $\mathbf{X}$.}

\section{PHYSORHINITES ( ${ }^{1}$ ).}

Front caréné, convexe; hanches postérieures fortement et anguleusement dilatées; tarses courts, leur $3 e$ article lamellé et plus ou moins dilaté, le $4 \mathrm{e}$ très petit.

Physorhinds.

Eschs. Silb. Rev. entom. IV, 1836.

Articles 2 et 3 des antennes petits, égaux; hanches postérieures triangulairement dilatées en dedans; front jaune, en général, quelle que soit la couleur du corps.

erythrocephalus FABR. Syst. El. II, p.241 . Amér. trop. insularis CAND. Élat. nouv. III, p. 58. . . . Guadeloupe. distigma CAND. Monogr. II, p. 390. . . . . Mexique. marginatus CAND. Ibid., p. 391. . . . . . Brésil. stellatus CAND. Ibid. . . . . . . . . Nouv. Grenade. sexnotatus StenNH. Col. Hefte XIV, p. 126 . ” sericeus CAND. Monogr. II, p. 392 . . . . . Mexique. xanthocephalus GERM. Zeitschr. II, p. 245 . Brésil. lateralis CANd. Monogr. II, p. 393 . . . . . Pérou. Sturmi Germ., loc. cit. . . . . . . . . Mexique.

(1) Cette tribu a été déplacée et mise à la suite des Elatérites, au lieu de précéder ces derniers, mesure déterminée par l'avantage de ne pas séparer les Heceroderes des Drasterius. 
frontalis CAND. Monogr. I, p. 394 . . . .

Mexique.

cephalicus CAND. Élat. nouv. IV, p. 31

)

galapagoensis WATERH. Ann. of. nat. Hist.

1845, p. 25 . . . . . . . Gallapagos.

\section{Porthnimius.}

Germ. Faun. Ins. Europ. 1848, p. 24, 7.

Articles 2 et 3 des antennes égaux, plus longs, réunis, que de 4 mo; hanches postérieures dilatées en dedans en lame quadrangulaire.

austriacus Schrank, Enum. Ins. Aust. 1781, p. 186.

Europe.

var. fulvus W. ReDt. Quæd. Gen. 1842 . . . ”

\section{ANCHAStUS.}

Leconte, Trans. Am. phil. Soc. X, 1853, p. 459.

Brachycrepis Lec. Crepidotritus Lec. Podeonius Kres (O. $\mathrm{im}$ ). Article 3 des antennes plus long que 2, souvent égal au quatrième; dilatation des hanches postérieure grande et subquadrangulaire.

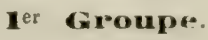

Europe.

acuticornis Germ. Ins. Sp. nov., p. 57 . Europe centrale. sanguinicollis var. Rossi, Faun., p. 402, note.

subcyaneus Kiesenw. Nat. Ins. IV, p. 2, 351 (note).

Grèce.

\section{ae Groupe.}

Continent et iles asiatiques.

Davidi CANd. Élat. nouv. II, p. 24 . . . C Chine centrale. sinensis CAND. Monogr. II, p.410 . . . . . Chine. aquilus CAND. Mém. Soc. Liége V, $2^{\text {ne }}$ sér. . J Japon. puncticollis CAND. Monogr. II, p.411. . . . Ceylan. flavus CAND. Élat. nouv. II, p. 24. . . . . ” 
pusio CAND. Monogr. II, p. 410

Ceylan.

humeralis CAND. Ibid. . . . . . . . . ”

infumatus CAND. Élat. nouv. I, p. 27 . . . . ”

lateralis CAND. Ibid., p. 28 . . . . . . . Siam.

Gastelnaui CAND. Tbid. II. p. 24. . . . . . ”

vulneratus CAND. Ibid. . . . . . . . "

unicolor CAND. Ibid. III, p. 61 . . . . . . Mindanao.

rufangulus CAND. Ins. des Philipp. in Soc.

belg. XVIII, Bull. 1875 . . . . . . . . ”

venustulus CAND. Élat. nouv. I, p. 27. . . . Bornéo.

sericeus CAND. Ibid. . . . . . . . . ”

saucius CAND. Ibid. IV, p. 31. . . . . . . ”

nitidus CAND. Ibid. II; p. 25 . . . . . . . ”

nigriceps CAND. Élat. nouv. II, p. 25 . . . . Java.

spectabilis CAND. Notes Leyd. Mus. II, p. 1 . Sumatra.

simulans CAND. Ibid. V, p. 210 . . . . . . 》

serdangensis CAND. Ibid. . . . . . . . »

nigripennis CAND. Ann. Mus. Gênes XV,p.193. ”

\section{3e Groupe.}

Afrique.

senegalensis CAND. Monogr. $\Pi$, p. 408 . . .

Sénégal.

laminatus CAND. Élat. nouv. IV, p.32 . . . Schoa.

Raffrayi CaNd. Ibid. III, p. 60 . . . . . . Abyssinie.

Klugi Cand. Ibid. . . . . . . . . . . Gabon.

pectoralis CAND. Ibid., p. 61. . . . . . . ”

ligneus CAND. Ibid. . . . . . . . . . ”

rutilus Kuvg, Erman. Reis. 1835, p. 31 . . . Guinée.

dubius KuđG, Monatsb. Berl. Acad., 1855, p.647. Mozambique. atlanticus CAND. Monogr. II, p. 409 . . . . Ste-Hélène.

puncticollis Woluast. (Heteroderes) Ann. a. Mag.

compositarum Wollast. Col. S. Helen.

sylvanus CAND. Élat. nouv.IV, p. 32. . . . Madagascar.

morio CAND. Ibid. 
a Groupe.

Australie et Mélanésie.

australis CAND. Élat. Nouv. II, p. 25

Victoria.

Albertisi Cand. Ann. Mus. Gênes XV, p. 191. Nouv. Guinée. longicornis CAND. Élat. nouv. I, p. 28 . . . ఎ nitidulus CAND. Ann. Mus. Gênes XII, p. 119 . ग pisciculus CAND. Ibid. . . . . . . ” tongaensis CAND. Élat. nouv. II, p. 25 . Viti, Tonga-tabou. major CAND. Ibid. . . . . . . . Viti.

\section{Se Groupe.}

Amérique septentrionale.

signaticollis Gerr. Zeitschr. V,p.190 . . Amérique bor. tantillus Mañ. Bull. Mosc., 1813, p. 239 . . Californie. cinereipennis Eschs. Thon Arch., 1829, II, I, p. 31

binus SAY, Trans. Am. phil. Soc. VI, p. 177 . Indiana. militaris CAND. Élat. nouv. III, p.59 - . . . Californie. asper Lec. Proc. Am. phil. Soc., 1878, p. 404 . Floride. longulus Lec. Ibid. . . . . . . . . ” fuscus Lec. Tbid. . . . . . . . . . ” bicarinatus LEC. Ibid. X, p. 46 . . . . . . ” bicolor Lec. Proc. Acad., 1866 . . . . . . Californie. desertus HoRN.

digitatus Lec. Trans. Am.ph. Soc., 1853, p.439. Pensylvanie. sericans Emend. sequent. . . . . . Arizona. sericeus Hons, Trans., 1871, p. 311.

frontalis Hors, Trans. Am. ent. Soc. XII, p.49. N. Mexique. rufus CAND. Monogr. II, p. 404 . . . . Nouv. Orléans. rufescens CAND. Tbid. . . . . . . . . Cuba. rufiventris CAND. Ibid., p. 401 . . . . . . » terminatus CAND. Elat, nouv. I, p. 28 . . Guadeloupe. suturalis CAND. Ibid. I, p. 27. . . . . . Mexique. 


\section{Ge Groupe.}

Amérique méridionale.

trisignatus Steinh. Col. Hefte XIV, p. 127. Nouv. Grenade. apicalis Steinh. Mith. Munch. entom. Ver., 1877, p. 83

niger Stennн. Col. Hefte XIV, p. 127 . . . . »

Candezei Sternh. Tbid., p. 126 . . . . . . ”

brevis CAND. Élat. nouv. III, p. 59 . . . . . ”

balteatus Cand. Tbid. II . . . . . . . . Bahia.

hilaris CAND. Monogr. II, p.400. . . . . Brésil.

phedrus C̣ND. Ibid. . . . . . . . . . ”

quadrimaculatus CAND. Élat. nouv. IV, p.32. ”

fasciatus CAND. Ibid. III, p. 58. . . . . . Para.

æoloides CAND. Ibid. II, p. 24 . . . . . . »

unitinctus CAND. Ibid. IV, p. 32 . . . . . . ”

alopex CAND. Thid., p. 33 . . . . . . . . ”

bitinctus CAND. (au lieu de bicolor) Élat. nouv.

III, p. 59.

\section{TRIBU XI.}

\section{POMACHШПTES.}

Front grand, convexe; prothorax souvent épaissi et élargi antérieurement; sutures posternales concaves et creusées en avant chez la plupart; corps long, étroit; le reste comme chez les Élatérites.

\section{Psinoniscus.}

CAND. Monogr. d. Élat. III, p. 3.

Conoderus Chevr.

Tarses simples; sutures prosternales fines et rectilignes; corps très grêle.

apicalis Cherr. Col. d. Mex. cent. II, n 198 . Mexique. 
borborurus CAND. Monogr. III, p. 5 . . . Mexique. sticticus CAND. Ibid., p. 6 . . . . . . . ” brunneus Cand. Tbid. . . . . . . . . ”

Anius.

CAND. Élat. nouv. IV, p. 37.

Caractères des précédents, mais les hanches postérieures étroites et non dentées au milieu.

gracillimus CAND., loc. cit.

Chili.

Deronecus.

Solier in GAY, Historia de Chile, Zool. V, p. 11.

Podonema et Mecothorax SoL.

Tarses simples; sutures prosternales concaves et creusées au sommet; fossette mésosternale à bords élevés; genre chilien.

section $\mathbf{E}$.

Articles 2 et 3 des antennes égaux.

impressus Sol., loc. cit., p. 13

Chili.

castaneipennis SoL. Ibid., p. 22

)

carinatus CAND. Élat. nouv. III, p. 72

)

tumidus CAND. Tbid.

)

pusillus Kirsch, Berl. ent. Zeits., 1870, p. 346.

Bogotre. profugus Kזrsch, Ibid., 1883.

\section{Section II.}

Article 2 plus long que $3 ; 4^{\Theta}$ article des tarses très petit.

rubricollis Sol. Hist. Chile V, p. 14 . . . . sanguinicollis CAND. Blat. nouv. $\amalg$, p. 30 .

Chili. thoracicus SoL. loc. cit.

attenuatus Sor. Ibid.

inops CAND. Élat. nouv. II, p. 29. 
brevicollis CAND. Tbid., p. 30 . . . . . . . Chili.

scapularis CAND. Tbid. . . . . . . . ”

debilis CAND. Ibid. . . . . . . . . . . »

Gection II.

Article 2 plus long que $3 ; 4^{\mathrm{e}}$ article des tarses normal.

vulgaris Sor. Hist. d. Chile V, p. 13 . . . . Chili.

cervinus CAND. Élat. nouv. III, p. 73 . . . . ”

suturalis CAND. Ibid. I. . . . . . . . . ”

angustatus Sor. loc. cit. . . . . . . . ”

fllicornis Sol. Tbid. . . . . . . . . ”

nigricornis CAND. Monogr. II, p. 15. . . . ”

umbilicatus CAND. Ibid., p. 16 . . . . ?

agriotes CAND. Élat. nouv. II, p. 30 . . . . ”

curtus CAND. Tbid., p. 31 . . . . . . . . ”

grisescens CAND. Ibid. . . . . . . . . ”

adrastus CAND. Tbid. . . . . . . . . . .

tenuicollis CAND. Ibid. III, p. 72 . . . . . ”

anchastinus CAND. Tbid., p. 73 . . . . . . ”

\section{section IV.}

Article 2 des antennes plus court que le troisième.

canaliculatus FAIRM. Ann. Fr. 1885, p. 45. Terre-de-Feu.

\section{MEdonta.}

CAND. Monogr. III, p. 17.

Bedresia pars SoLIER.

Pomachiliites à $4^{0}$ article des tarses bilobé, lamellé.

punctolineatus SolIER, in GAY, Histor. de

Chile V,p.25 . . . . . . . . . . Chili.

Fairmairei CAND. Élat. nouv. II, p. 31 . . . ” 


\section{SOMOMECUS ( $\left.{ }^{1}\right)$.}

Sol. in. GaY, Hist. Chile V, 1851, p. 33.

Plaque nasale obsolète.

parallelus Sou. loc. cit., p. 33

Chiti.

\section{BETARMON.}

Kresenw. Nat. Ins. IV, p. 2, 265.

Tournure et caractères des Agriotes, avec le front rebordé en avant; 3 e article des tarses dilaté.

bisbimaculatus FABR. Ind. alph. 1803, p. 293.

Italie. quadrimacula Hersst, Käf. quadrimaculatus FABR. Entom. Syst.

picipennis BacH, Kaferf. II, 1852, p. 32 (Agriotes) Allemagne. styriacus RedT. Fn. Austr. $2^{e}$ éd., p. 512.

vittatus CAND. Entom. Monatsblatter, 1880. Asturies. anatolicus CAND. Élat. nouv. III, p. 74 . . . smyrne. Scharpi CAND. Ibid. . . . . . . Nouv. Zélande. frontalis Sharp, Ann. and Magaz. may 1877 . » gracilipes Sharp, Ibid. . . . . . . . ” latus SHarp, Tbid. . . . . . . . . . » obscurus SHARP, Ibid. . . . . . . . »

Protelater.

Sharp, Ann. and Mag. may 1877.

Front d'Eucnémide, c'est-à-dire recourbé en avant pour s'épanouir en une plaque nasale triangulaire, encadrant de grandes fossettes antennaires.

elongatus Sharp, loc. cit. . . . . Nouv. Zélande. Huttoni SHarp, Ibid. . . . . . . . . ” guttatus SHARP, Ibid. . . . . . . . . . »

(1) Ce genre n'est maintenu à cette place qu'à cause de son analogie étroite avec les Deromecus; il pourrait aussi bien être relégué non loin tles Corymbitites. 
nigricans Sharp, Ibid. . . . . Nouv. Zélande. picticornis SHARP, Tbid. . . . . . . . . ” opacus SHARP, Tbid. . . . . . . . . ”

Pansp жus.

Sharp, Ann. a. Magaz. 1877, p. 15.

Intermédiaire entre les Betarmon et les Cryptohypnus. guttatus Sharp, loc. cit. . . . . . . Nouv. Zélande. tenebrosus Broun, N. Zeal. I. Sc. 1880 . . . »

Stibadoderds.

Burm. Stett. Ent. Zeitschr. 1875.

Sutures prosternales fines; tarses simples.

murinus Burær, loc. cit. . . . . . Répub. Argentine.

SMrticerus.

CAND. Monogr. III, 1860, p. 20.

Antennes à articles très larges à partir da 40 ; troisième article des tarses dilaté.

bitinctus CAND. Monogr. II, p. 22. . . . . N. Grenade. Sallei CANd. Ibid. . . . . . . . Vénézuéla. zonatus CAND. Élat. nouv. II, p. 74 . . . . Bogota. Belti Sharp, Monthl. Mag. 1880, XVII, II. . Chontalès.

Eschatroxus.

CAND. Élat. nouv. I, 1865, p. 37.

Quatrième article des tarses bilobé, lamellé.

holosericeus CAND., loc. cit. . . . . . . Moluques.

Pomachitus.

Eschs. Thon, Arch. 1829, II, 1,.p. 31.

Pomatochilus Gem. et Har. Cat.

Troisième article des tarses élargi, cordiforme, parfois lamellé ; bords de la fossette mésosternale saillants. 


\section{section I.}

Elytres épineuses à l'extrémité.

centrurus CAND. Monogr. II, p. 27

Brésil.

ocellatus CAND. Élat. nouv. II, p. 32

melanurus CAND. Monogr. III, p. 27

)

flavipes CAND. Ibid.

brunneus CAND. Ibid., p. 28

crassiusculus CAND. Ibid.

signatus CAND. Ibid., p. 30

subfasciatus GERM, Ins. Sp. nov., p. 50

hallomenus CAND. Monogr. III, p. 31.

æoloides CAND. Ibid., p. 31

nigriceps Erichs. Wiegm. Arch., 1847, I, p. 77.

frontalis Caxd. Monogr. III, p. 32 . . . . Nouv. Grenade.

atriceps Steinh. Col. Hefte XIV, p. 128.

suturalis CAND. Monogr. III, p. 33.

))

cuspidatus CAND. Ibid.

Vénézuéla.

ligneus CAND. Ibid., p. 34 . . . . . Nouv. Grenade.

macilentus CAND. Ibid., p. 35

guttatus CAND. Ibid.

Brésil.

collaris CAND. Ibid., p. 36 .

suturella CAND. Ibid.

. Nouv. Grenade.

\section{Gection II.}

Elytres échancrées ou tronquẻes au sommet; sans épine.

carmelita Cand. Monogr. III, p.37 . . . Nouv. Grenade. alticola Sternн. Col. Hefte XIV, p. 129 .

terminatus CAND. Monogr. III, p. 37.

Brésil.

melanocephalus CAND. Ibid., p. 38

nigrifrons Sterni. Col. Hefte XIV, p. 129. Nouv. Grenade. granulipennis CAND. Monogr. III, p.39.

linearis CAND. Ibid.

longicollis Steinh. Col. Hefte XIV, p. 130 .

filiformis CAND. Élat. nouv. IV, p. 38 . 
hiosurus CAND. Monogr. III, p. 40. . . . . Brésil. scapularis CAND. Ibid. . . . . . . Nouv. Grenade. minutus CAND. Ibid., p. 41. . . . . . . . Guyane. semicolon Stenn. Col. Hefte XIV, p. 130. Nouv. Grenade. canaliculatus Krrsch, Deutsch. ent. Zeitsch.,

1883

nanus CAND. Monogr. III, p. 41. scenicus CaND. Ibid., p. 42.

"
Bahia.
Rio.
"
Brésil.
"
"
"
"
"
"

histrio CAND. Tbid., p. 43

trifasciatus $\mathrm{C}_{A N D}$. Ibid.

interruptus $\mathrm{C}_{\mathrm{AND}}$. Tbid.

graphypterus CAND. Ibid., p. 44

lineolatus CAND. Tbid.

titillatus CAND. Ibid., p. 45

palliatus CAND. Tbid.

inquinatus CAND. Ibid., p. 46.

fragilis CAND. Ibid., p. 47

pileatus CAND. Tbil.

\section{section II.}

Elytres mutiques et entières au sommet.

ornatus CAND. Monogr. III, p. 48

Brésil.

pulchellus CAND. Ibid.

)

pumilus CAND. Ibid., p. 49 .

- )

\section{TRIBU XII.}

\section{CRYPTOHYPNITES.}

Front grand, rebordé; prosternum large, généralement dilaté au milieu, ses sutures fines ou effacées; hanches postérieures brusquement dilatées en dedans. 


\section{Monadious.}

Cand. Monogr. III, p. 52.

\section{Cryptohypnus GERM.}

Quatrième article des tarses dilaté et sublamellé; tête mobile dans le prothorax.

bilæsus Germ. Zeitschr. V,p. 145

Brésil. emys CAND. Monogr. III, p. 54 . . . . . ” mobiliceps CAND. Ibid. . . . . . . . ” brunnipennis CAND. Élat. nouv. I, p. 38. . . Cayenne. humeralis SteinH. Col. Hefte XIV, p. 131. Nouv. Grenade. quadrinotatus STEпн. Ibid., p. 130 . . . . ”

\section{CRYPTOHYPNUS.}

Eschs. in Silberm. Revue entom. IV, 1836 (tableau). Aplotarsus Curtis, Cryphthypmus Kies. Hypnoidus Steph. Hypolithus Esch. Scoliocerns Mots. Zorochrus THомs. Negastrius Thомs.

Tarses simples; sutures prosternales visibles dans toute leur longueur.

\section{1er Groupe.}

Europe et Sibérie (').

hyperboreus GruL. Ins. suec. IV, p. 350. Laponie, Sibérie, Amér. arct.

planatus Eschs. Th. Arch.

depressus GeBL. Bull. Mosc. 1847 IV, p. 420 . Sibérie. nitidicollis KönIa, Hor. Soc. Ent. Ross. 1889 . Asie centrale. maritimus CunTis, Ann. nat. Hist. 1840 . . . Europe. scotus CAND. Monogr.

( $\left.{ }^{2}\right)$ Quelques espèces sont communes à la Sibérie et à l'Amérique boréale. Je les ai disposées ici suivant leur centre principal ; c'est ainsi que l'hyperboreus est considéré comme sibérien, bien qu'on le rencontre en Amérique et les littoralis et bicolor, qui ont des représentants au Kamschatka, méritent d'être rangés dans les espèces américaines. 
gracilis Muls. Opusc. VI, p. 30 . . . . Eur. centrale. morio Kresenw. Nat. Ins. IV.

arcticus CAND. Monogr. III, p.66 . . . . . Laponie. barbatus SАнLв. Veg. Expl. 1884 . . Penins. Tschuktcha. riparius FABR. Ent. syst. 1, 2, p. 235 . . . . Europe. aneus Marsh. Ent. brit.

littoreus HERBst, Käf. politus $\mathrm{F}_{\mathrm{ABR}}$., loc. cit.

Meyeri Stierd. Mitth. Schw. Ent. Ges. 1862,

p. 36 . . . . . . . . . . Shiisse. gibbus GEBL. Bull. Mosc. 1847, p. 421. . . . Sibérie. rufescens GEBL. Tbid., p. 423. . . . . . . Altaï. rufotestaceus CAND. Monogr.

rivularius Grul. Ins. suec. I, p. 403 . . . . Europe bor. frigidus Kresenw. Nat. Ins. IV, 2, p. 361 . . Styrie. canaliculatus GeBL. Bull. Mosc. 1841, p. $583 . \quad$ Altä̈. Balassogloi CANd. Élat. nouv. IV, p. 38 . Ferghana. quadripustulatus FABR. Entom. System.

I, II, p. 236 . . . . . . . Europe, Amér.bor. agricola Zetтenst. Act. Holm. 1824.

quadrum Grut. Ins. suec. IV.

tenuicornis GerM. Insp. Sp.nov., p. 60 . . . Allemagne. consobrinus Muls. Opusc. VI, p. 30.

elongatus REDT. Faun. Austr. minusculus BACH, Käf. III, p. 35.

pulchellus Lovse, Syst. Nat. 1, 2, p. 656. Europe, sibérie. var. trimaculatus $\mathrm{F}_{\triangle \mathrm{BR}}$. Ent. syst.

sabulicola Boнem. Vet. Ak. Handl. 1852, p. 74 S Suède. curtus GERMr. Zeitschr. V, p. 141 . . . . . Europe mér. alysidotus KIESENw. Nat. Ins. IV, 2, p. 368 . Dalmatie, Vénétie, Lombardie. boreaphilus THоNs. Skand. Col. 1864, p. 112. Suì̀de. algidus $\mathrm{S}_{\triangle H}$ B. Midd. Soc. Fenn. (Negastrius) IX, p. 28. . . . . . . . . . . . Laponie. 
tetratoma Rosenh. Thier. Andal. 1856, p. 136: Andulousie. välesiacus Stiert. Mitt sw. Ent. Gesels., p.18. Suisse. quadriguttatus CasT. Hist. nat. Ins. II, p.245. Europe. quadripustulatus РАYK. STEPH.

tetragraphus GERM. Zeitschr. V.

angularis CAND. Col. Hefte V, 1869 (Emend.). Espagne. exilis CAND. Monogr. II, p. 84.

dermestoides Herbst, Käf. X, p. 65 . . . . Europe.

flavipes AđBÉ, Ann. Fr., 1850.

minimus LAC. Faun. Par., p. 660.

quadripustulatus var. GrLt. Ins. suec.I, p.424.

meridionalis CAST. Hist. nat. II, p. 246 .

)

lapidicola Gers. Zeitschr. V, p. 144.

var. pumilio KIEsEnw. Nat. Ins.

crux Küst. Käf. 18, p. 15 . . . . . . Sardaigne.

propinquus Desbr. Abeille VII, p. 106 . . . Corse.

minutissimus GerM. Faun. Ins. Eur., VI.

tabl. 8 . . . . . . Europe, Sibérie, Japon.

liliputanus GERM. Zeitschr. V, p.145. . . . sicile.

2e Groupe.

Himalaya, Chine et Japon.

davidianus CAND. Élat. nouv. III, p. 75 . . Chine centrale. perpusillus CAND. Ibid. II, p. 33. . . . . . Himalaya.

atomarius CAND. Ibid. III, p. 76 . . . $\because . . \quad$ » cruciatus CAND. Mém. Soc. Liége $\nabla, 2^{\circ}$ sér.

p. 11

quadrillum CAND. Ibid.

albipilis CAND. Ibid., p. 12.

curatus CAND. Ibid.

insulsus CAND. Tbid., p. 13

humeralis CAND. Ibid.

ovalis CAND. Ibid., p. 15.

ellipticus CAND. Ibid., p. 14

Japon. 
luteipes CAND. Ibid., p. 15.

Japon.

telluris Lewis, Ent. Monthl. Mag. Déc. 1879 .

3. Sroupe.

Amérique du Nord extratropicale ( ${ }^{1}$ ).

squalidus Lec. Am. Phil. Trans. X, p. 487 . Californie. planatus Lec. New. Sp. Col. I, 1863, p. 84 . . New-York. funebris CAND. Monogr. III, p.62 . . . . . Californie. Sanborni Horn, Trans. Am. ent. Soc., 1871,

p. 303. . . . . . . . . . . . . N. Hampshire. grandicollis Lec. New. Sp. Col., 1863, p. 83 . Canada. littoralis Eschs. Tном, Arch. II, I, p. 34. . . Sitkha. restrictulus MaNNERH. Bull. Mosc., 1853, III,

p. 228.

Pen. Kenaï.

impressicollis MaNn. Ibid., p. 225.

)

abbreviatus SAY, Journ. Acad. Phil., 1823,

p. 73 .

Missouri. silaceipes Germ. Zeitschr, CAND. Mon.

bicolor Eschs. Thол Arch. II, I, p. 33. . . Amér arctique. piscescens Lec. Trans. X, p. 486.

scarificatus, limbatus, lucidulus, fallax MANNERH.

Bull. Mosc.

nocturnus EscHs., loc. cit.

lacustris Lec., CAND., loc. cit.

musculus EsoHs. Entomogr. I, p. 70 . . . . Unalaschka. tumescens Lec. Trans. Am. Soc. X, p. 486. Lac Supér. dubius Horn, Trans, Am, entom. Soc., 1871,

p. 305. . . . . . . . . . . . Nebraska. striatulus Lec. Ibid., p. 488 . . . . . . . Lac Supér: exiguus RAND. Bost. Journ. II, p. 35 . . . . Pensylvanie. guttatulus MeLsH. Proc. Ac. Phil. II, p. 214.

(') Quelques indications utiles m'ont été données par le dr IIorn, qui prépare un travail sur les Cryptohypnus des États-Unis. 
choris SAr, Trans. Am. phil. Soc. VI, p. 172 . Etats-Unis. ornatus LEc. Tbid. X, p. 487 . . . . . . . Californie.

var. colon HorN, loc. cit.

gentilis Lec. Proc. Acad. Phil., 1866, p. 389 . Nebrastia. pectoralis $\mathrm{SAY}_{\mathrm{Y}}$ loc. cit.

Missouri.

var. futilis Lec. Trans. X, p. 488 . . . . . Californie.

var. inops Lec. Ibid. . . . . . . . . . ”

obliquat ulus Mersh. Proc. Ac. Phil. II, p. 214. Pensylvanie. æstivus Hork, Trans. Am. entom. Soc., 1871,

p. 304 .

Missouri.

perplexus Hons, Tbid.

Pensylvanie.

\section{Groupe.}

Espèces africaines.

fulvus CAND. Élat. nouv. $\Pi$, p. 33 . . . . . Abyssinie. binodulus Germ. Abhandl. Berl. Acad., 1832, p. 68 hova CAND. Élat. nouv. IV, p. 38.

Madagascar.

)

\section{Se Groupe.}

Espèces intertropicales.

ænescens Мотsсн. (') Etud. Ent. 1858

Birmanie.

exilis Мотsсн. Ibid.

)

indicus MOTsCH. Tbid.

))

fasciatus CAND. Élat. nouv. I, p. 38 . . . .

Bornéo. suturalis CAND. Ann. Mus. civ. Gênes XII,

p. 131.

Nouv. Guinée.

javanus CAND. Ibid. Java.

misellus Bонем. Eugen. Resa, 1858, p. 69 ) squamifer CAND. Élat. nouv. I, p. 38 . Para. Oberthuri CAND. Ibid. III, p. 75. Nouv. Grenade.

(1) Cette espèce ainsi que les deux suivantes sont peut-être des Arrhaphes el Hemirrhaphes. 


\section{G Groupe.}

Australie et Nile Zélande.

variegatus MAC LEAY, N. S. W. Trans. II,

p. 257. . . . . . . . . . Queensland. flavicans CAND. Élat. nouv. IV, p. 38. . . . Melbourne. frontalis Sharp, Ann. a. Mag. May 1877,

p. 22 . . . . . . . . . Nouv. Zélande.

humilis Sharp, Ibid. . . . . . . . . »

longicornis SHARP, Tbid. . . . . . . . ”

Powelli Sharp, Tbid. . . . . . . . ”

thoracicus SHARP, Ibid. . . . . . . . . ”

montanus Broun, New Zeal. J. S., 1882, p.302. ”

Sandageri Broun, Ibid. . . . . . . . . . ”

Meinertzhageni Broun. Tbid., p. 680 . . . ”

Ye Groupe.

Amérique méridionale extratropicale.

nivalis FATRM. Col. Chil., 1860 . . . . . .

andicola FArRM. Ibid. . . . . . . . . . »

atomus CAND. Élat. nouv. II, p.34 . . . . . ”

æqualis CAND. Ibid. III, p. 76 . . . . . Uruguay.

Chili.

)

\section{Arctaptha.}

PANDELlí, in litt.

Hanches postérieures moins dilatées en dedans que chez les Cryptohypnus, dont il ne diffère en réalité que par un facies d'Athous et une inégalité considérable de taille entre les sexes.

Brucki (Cryptohypnus) CAND. Élat. nouv. I, p. 38. Pyrénées.

AMPHFLATys.

Sharp, Ann. a. Mag. may 1877, p. 12.

Antennes à articles 4-10 élargis, dentés de chaque côté.

Lawsoni Sharp, loc. cit. . . . . . . Nouv. Zélande. 


\section{ArRHAPHES. \\ CANd. Monogr. III, p. 98. \\ Phorotarsus Mots. Bull. Mosc. 1861. \\ Prosternum sans sutures latérales.}

diptychus CAND., loc. cit

Ceylan. picturatus Mots., loc. cit.

minusculus CAxd. Élat. nouv. II, p. 33 . . Birmanie. opacus CAND. Ibid. . . . . . . . . . . Himalaya. Gestroi CAND. Ann. Mus. Gênes XII, p. 131 . Java. nigriceps CAND. Ibid. XV, p. 194 . . . . Sumatra.

\section{HEMTRRAPHES.}

CAND. Élat. nouv. П, p. 32.

Sutures latérales du prosternum incomplètes.

notabilis CAND., loc. cit. . . . . . . . Birmanie.

\section{Oedostethus.}

LEC. Trans. Am. phil. Soc. X, p. 489.

Crochets des tarses fortement dentés.

femoralis LEC, loc. cit. . . . . . . Etats-Unis sept.

Pseudiconus.

CAND. Élat. nouv. II, p. 76.

Hanches postérieures à peine élargies en dedans.

mendax CAND., loc. cit., p. 77.

Chiti.

\section{TRIBU XIII.}

\section{CARDIOPHORITES.}

Front étroit, rebordé en avant; écusson cordiforme; prosternum large, parallèle, sa saillie courte; fossette mésosternale à bords verticaux. 


\section{Coptostethos.}

Wollast. Ins. Mader. p. 238.

Aptères; hanches postérieures réduites à une grande lame. Vivent généralement sous les pierres.

canariensis WoLLAst. loc. cit. . . . . Madère. femoratus WoLL. Ibid. . . . . . . . : . ” crassiusculus WoLl. Cat. Canar., 1864. . . I. Canaries. globulicollis WoLl. Ann. Nat. Hist. 1862, p.440. Ténériffe. gracilis WoL. Cat. 1864 . . . . . . . . ” obtusus Wor. Tbid. . . . . . . . . . ” brunnipennis WoLL. Ann. nat: Histor., 1863 . ” basilaris CAND. Élat. nouv. I, p. 40 (Cardioph.). Cap. guttatus CAND. Tbid., p. 39 . . . . . . . ” sexpunctatus CAND. Ibid. II, p. 34 . . . . ” majusculus CAND. Tbid. IV, p. 39 . . . . . is flavus CAND. Tbid. . . . . . . . . . . . " . . tenuipes CAND. Ibid. . . . . . . . . . . ” americanus HorN ('). Tr. am. ent. Soc. 1871, p. 303.

Cardiophorús.

Eschsch. Thon, Arch., 1829, II, 1, p. 34.

Caloderus Steph.-Dicronychus BRuLL. - Gauroderus ThомS. Platynychus pars Mотs.

Dos du prothorax non séparé des flancs par une arête entière.

\section{Ier froupe.}

Europe, Sibérie, Chine, Japon, région méditerranéenne.

a. Crochets simples.

(') Le genre est indiqué avec un signe de doute par l'auteur. 
thoracicus FABR. System. Entomol, p. 214 .

Europe. gramineus Scop. Ent. carniol.

ruficollis Sohrank, Enum. Ins.

miniaticollis CAND. Monogr. III, p. 122.

Grèce.

syriacus LINNÉ, System. Nat. 1, 2, p.65̃3 . Asie mineure. pictus FALDERMr. Faun. transe.

Hinkei Frivaudsky, A Magg. Tudòs.

maculicollis Reiche, Ann. Fr. 1856 . . . . Grèce.

ægyptiacus CAND. Monogr. III, p. 125 . . . Egypte.

Gandezei Könı, Hor. Soc. Ross, 1889. . . . Asie centr.

discicollis Herbst, Käf. X, p.92 . . . . . Europe or.

volgensis Fisch. Ent. Ross.

sacratus Errchs. Mon., p. 284 . . . . . . Syrie.

nigricornis BAUDI, Berl. Z., 1859, p. 342.

Kindermanni Cavo. Monogr. III, p. 128

)

ruficollis LrNwé, Syst. Nat. 1, 2, p.655 . . . Europe.

collaris Errchs. Monogr., p. 983 . . . . . Italie.

nigricollis ERICHS. Ibid. . . . . . . . Bockhara.

longulus ERICHS. Ibid., p. $306 . \quad$. . . . . ”

Olgæ Solski, Hor. Soc. ent. Ross, 1881, p. 233. Samarcande. blandus Solski, Ibid.

picticollis KraAtz, Deutsch. Entom. Zeitschr.,

1881, p. 320.

ulcerosus GÉNÉ, Mém. Ac. Tur., 1836, p. 175 . Sardaigne. numida CAND. Monogr. III, p. 132. . . . Barbarie.

Lethierryi Desbr. Abeille, 1887.

argiolus GÉNÉ, loc. cit. . . . . . Sardaigne, Espagne.

hipponensis Dessr. Abeille, 1870.

anticus Erichs. Monogr., p. 285 . . . . Iles Ioniennes.

signatus Ox. Ent. I, p. 31, 1790 . . . . . Espagne.

sexpunctatus Latr. Hist. Nat. Ilug Mag.

sexmaculatus Luc. Expl. Algérie.

Gertschmani CAND. Entom. Monatsbl., 1880. Asturies.

Gebleri CaND. Monogr. III, p. 137 . . . . Altaï. 
biguttatus OL. Entom. II, 31, p. 47 . . . . Europe mér. ornatus CAND., loc. cit.

pictus CAST. Hist. Nat. Ins. I, p. 249.

submaculatus CAST. Tbid.

Farinesi VuLLA, Col. Eur., 1838.

bipunctatus FABR. Ent. Syst. suppl., p. 140 . ”

scapulatus CAND. Élat. nouv. $\Pi$, p. 34 . . . Tunisie.

nigropunctatus CA.vd. Monogr. III, p. 137 . Russie mér. Kaiserlingi KöNı, Hor. Soc. ent. Ross., 1889. Turkestanor. Eleonoræ Gíné, Mém. Ac. Tur., 1836 . . . Sardaigne. rufipes Fourcr. Entom. Paris, p. 38 . . . . Europe. vestigialis ERICHs. Monogr., p. 293 . . . . ” pallipes BrdLuÉ, Exp. Morée.

sequens CAND. Mém. Liége $V, 2^{r}$ sér.,p.16. Japon. tenellus Reiche, Ann. Fr., 1856, p. 421 . . . Orient. procerulus Krrs. Berl. Zeitschr., 1859, p. 21. Iles grecques. Kiesenwetteri CAND. Monogr. III, p. 156.

abdominalis AUBE, Ann. Fr., 1850, p. 337. . Barbarie. ruficruris Brullé, Exp. Mor. III, p. 140 . . Grèce. tibialis Entchs. Monogr., p. 295.

nigerrimus Erichs. Monogr., p. 296 . . . . Europe. Graëllsi CAND. Monogr. III, p. 160. . . . Espagne. longicornis SchaUfF. Ann. Fr., 1862. melampus Irur Mag. VI, p. 8. . deflexus. Schauff., loc. cit.

mauritanicus MотsсH. Etud. entom., 1858 pullatus CAND. Mém. Liége $\nabla, 2^{*}$ sér., p. 16 Algérie. Japon. ebeninus Germ. Ins. Sp. nov., p. 58 . . . . Europe. turgidus Erichs. Monogr., p. 259 .

Orient. rotundicollis Frivalds. A' Kival Mag., 1845. rudis CAND. Élat. nouv. III, p. 80 . . . : thebaicus CAND. Monogr. III, p. 177 . Nubie. Égypte. atramentarius ERICHS. Monogr., p. 297 . . Europe mér. megathorax FALD. Faun. transe. 
musculus Erichs. Monogr., p. 299

Europe.

advena FABR.?

curtulus Muls. Ann. Lyon, 1855.

mus CAND. Élat. nouv. IV, p. 40

Sibérie mér.

granarius CAND. Ibid.

Chine.

subulipennis Fatderm. Mém. Pétersb., 1835.

)

curtulus Mots. Schreink Reise, 1860, p. 112 .

Sibérie.

vulgaris Moтs. Tbid.

))

humilis EricHs. Monogr., p. 318 . . . . . Nubie.

asellus Erichs. Tbid.

Europe. inflatus CAND. Élat. nouv. III, p. 78 . . . Mandtchourie. erythrurus CAND. Ibid. . . . . . . Chine centr. exaratus Erichs. Monogr., p. 304. . . . Espagne. Henoni CAND. Élat. nouv. IV, p. 39 .. . Constantine. adjutor CAND. Mém. Liége $\mathrm{V}, 2^{\circ}$ sér., p. 17 . . Japon. pauper CAND. Ibid.

rameus Lewis, Entom. Monthl. Mag. 1879.

)

brunneus ERICHS. Monogr., p. 307.

)

Nubie: dilutus ERICHS. Ibid.

)

permodicus FALD. Faun. Transc. 1, p. 172. . Arménie.

crux Erichs. Monogr., p. 291. . . . . . . Nubie.

obesus KrXnick. Bull. Mosc. 1832 . . . . . Charkov.

margelanicus HEYD. Deuts. Ent. Zeits. XXV, 1881, p. 325.

Turkestan.

$b$. Ongles dentés.

cinereus Herbst, Fuessl. Arch. V, p. 114 . Europe.

testaceus FABR. Ent. syst.

obesus BruLxi, Exp. mor.

pilosus PAYK. Faun. suec. III, p. 25.

Weberi WaLTL, Isis, 1838.

versicolor Muls. Opusc. Entom. VI, p.96. . France mér. asperulus CAND. Monogr. III, p. 194.

agnatus $\mathrm{CAND}_{\mathrm{AND}}$ Ibid., p. 189

Europe. 
incanus Erichs. Monogr., p. 311. ... . . . Sardaigne. crassicollis EricHs. Ibid. . . . . . . . Crimée. geminatus Erichs. Tbid. . . . . . . . . ” hæmatomus CAND. Monogr., p. 198 . . . . Algérie. decorus FatD. Fn. Transc. 1, p. 179 . . . . Arménie. vexillarius CAND. Élat. nouv. IV, p. 44 . . Sibérie. equiseti Herbst, Füessl. Arch. V, p. 114 . . Europe. luridipes Lacond. Fn. Par. filiformis Rossi, Fn. etrusc. pilosus HerBst, Käf.

dahuricus CAND. État. nouv. IV, p. 44 . . . Daurie. rubripes Germ. Ins. Sp. nov., p. 59 . . . . Europe mér. pollux GERM. Ibid. messenicus Bruli. Exp. Mor.

extinctus Erichs. Monogr., p. 317. . . . . Mésopotamie. febriens CAND. Monogr. III, p.197. . . . . . Egypte. davidianus CAND. Élat. nouv. II, p. 80 . . . Chine centr. devius CAND. Ibid., p. 81 . . . . . . . ” nothus CAND. Ibid. I, p. 43. . . . . . . Japon.

Note. Auy quels il faut ajouter les espèces suivantes de M. Desbrochers des Loges, que je n'ai point vues et dont je ne connais pas la valeur spécifique: maculicrus, pusillus (Corse)breviatu.s, annulicornis (Ėspagne), concolor (Russie mér.), caucasicus (Caucase), Senaci (Bulgarie), convexithorax (Syrie), mauritanicus (Algérie).

\section{Groupe.}

Asie intertropicale ('), Malaisie.

a. Ongles simples.

lectus CAND. Monogr. IH, p. 125 . . . . Hindoustan. marginalis CAND. Ibid., p. 138 . . . . " " tetradicus CAND. Ibid.. . . . . . . . Bengale.

(1) Cette désignation ne doit pas ètre prise trop à la lettre; les espèces de tout l'Hindoustan, de toute l'Indo-Chine et même des points les plus méridionaux de la Chine y étant comprises. 
ophidius CAND. Ibid., p. 139 . . . . . . Nilgherries. notatus FABr. Spec. Ins. I, p. 273 . . . . . Coromandel. festivus Thunb. Nov. Ins. Sp.

eximius CAND. Monogr. III, p.140. . . . Népaul. anaticus CAND, Ibid. . . . . . . Hindoustan. formosus CuRTIS (forte).

carduelis CAND. Élat. nouv. I, p, 39 . . . Singapore. mirabilis CAND. Monogr. III, p. 141 . . . . Hindoustan. argutulus CAND. Ibid., p. 142 . . . . . . ” mesopyrrhus CaND. Ibid. . . . . . . ” nuptus CAND. Ibid., p. 153. . . . . . . . » obliquus CAND. Élat. nouv. I, p. 40. .. . . Ceylan. Dohrni CAND. Ibid. IV, p. 40 . . . . . . . 》 subæneus CaNd. (Horist). Monogr. III, p. 247. pallipes FABR. Mantiss. 1, p. 174 . . . . Hindoustan. umbraculatus Cand. Bull. Soc. entom. Belg. 1890

Bengale. soricinus CAND. Élat. nouv. I, p. 41 Birmanie. vagus CAND. Ann. Gênes 1888, p. 679. ) elegans CAND. Ibid. XII, 1878, p. 132. Célèbes. fasciatus CAND. Bull. Soc. Belg. XVIII, 1875. I. Phitipp. unicolor CAND. Ibid. ) lignipennis $\mathrm{C}_{A N D}$. Elat. nouv. IV, p. 40 .

Ternate.

b. Ongles dentés.

comptus Cand. Monogr. III, p. 202. Mysore. contemptus Cand. Ibid. Bengale. spissus CAND. Élat. nouv. I, p. 42 . . . . . Madras. servilis CAND. Monogr. III, p. 203 . . . . Bengale. astutus CANd. Ann. Gênes, 1888, p. 681 . . . Birmanie. nebulosus CAND. Monogr. III, p. 204 . . . . Bengale. bengalensis CAND. Élat. nouv. $\Pi$, p. 38 . lacertosus Erichs. Monogr., p. 316 Hindoustan. humerosus CAND. Monogr. III, p. 206. 
Moorei CAND. Ibid.

Madras. quadrillum CAND. Ibid. 207 . . . . . . . Hindoustan. madidus CAND. Bull. Soc. entom. Belg., 1890. Bengale. conductus ERICHs. Monogr., p. 316 stolatus ERrCHs. Ibid. Hindoustan. limbatus CAND. Monogr. III, p. 209 ) æquabilis CAND. Tbid. fuscipennis CAND. (Horistonotus) Ibid., p. 247. nitidus CAND. Elat. nouv. II, p. 38 .

Ceylan. javanus CAND. Monogr. III, p. 205. Ind̉es sept. melanopterus CAND. Élat. nouv. $\Pi$, p. 38 . . Camboge. Helferi CAND. Ibid. I, p. 42 . . . . . Birmanie. addictus CAND. Ann. Gênes, 1888, p. 680 . . ” vulneratus CAND. Tbid., p. 682 . . . . . . ” malitiosus CAND. Ibid. . . . . . . . . » pallidulus CAND. Ibid., p. 680 . . . . . . ” Doriæ CAND. Ibid., p.683 . . . . . . . ” manuleatus CAND. Ibid., p. 681. . . . . . ” Fairmairei CAND. Tbid., p. 680. . . . . ” rubicundus CAND. Bull. Soc. entom. Belg., 1890 Bengale. sobrinus CASt. Hist. Nat. Ins. I, p. 250 . . . Hindoustan. systenus CAND. Monogr. III, p. 210 . . . . ” bucculatus CAND. Ibid., p. 211 . . . . . . ” gangeticus CAND. Tbid., p. 212 . . . . . ” erythronotus CAND. Ibid. . . . . . . ” oxypterus CAND. Ibid., p. 213 . . . . . . ” fulvivellus CAND. Ibid., p. 214 . . . . . . ” anceps CANd. Bull. Soc. ent. Belg., 1890. . . Bengale. venaticus CAND. Monogr. III, p. 214 . . . . Java. rubiginosus CAND. Notes Leyd. Mus., 1880 . Sumatra. inconditus CAND. Bull. Belg. XVIII, p. 125. I. Philippines. spernendus CAND. Ibid. . . . . . . . . » papuensis CAND. Ann. Gênes XII, p. 133 . Nouv. Guinée: 
consentaneus KoLl. (') Hugel, Kashm. IV, p. 2, 508. vicinus Kolr. Ibid.

Cachemire. indicus Мотsсн. Etud. entom. 1858, p. 59 . . Hindoustan. crucifer Mотs. Tbid. humeralis Мотs. Tbid. . . . . . . . . Ceylan. lateralis Mots. Bull. Mosc., 1861 . . . . . . . pictus Mотs. Etud. entom. 1858. . . . . . ” quadrimaculatus Mots. Bull. Mosc. 1861 . . ” humerifer WALKER, Ann. Nat. Hist. 1858 . . ” luzonicus Eschs. Thon, Arch. II, 1, p. 34 . Luçon.

\section{3e Groupe.}

Afrique intertropicale et australe et ses Iles. - Arabie $\left(^{(3)}\right.$.

a. Afrique orientale, Abyssinie (y compris l'Arabie) jusqu'au Zanguebar inclusivement.

Hedenborgi CAND. Monogr. III, p. 197 . Sennaar, Arabie. flavus Er. Monogr., p. 304. . . . . . . . Arabie. inquinatus CAND. Monogr. II, p. 198 . . . Abyssinie. insignis CAND. Élat. nouv. II, p. 35 . . . . ” æthiopicus CAND. Ibid., p. 36 . . . . . . » jocosus CAND. Tbid. III, p. 79. . . ... . . ” gagatinus CAND. Ibid., p. 80 . . . . . . ” funebris CAND. Ibid. IV, p. 42 . . . . . ” abyssinus CAND. Ibid., p. 43 . . . . . . ” paternus CAND. Ibid. . . . . . . . . » munitus CAND. Ibid. . . . . . . . » Amari CAND. Ibid. . . . . . . . ” culciarius CAND. Ibid. . . . . . . . . ”

(1) Je n'ai point vu les types de cette espece et des neuf qui suivent en sorle que, ne sachant si elles ont, ou non, les ongles dentés, je ne puis leur assignerleur place dans l'une ou l'autre section.

(a) Au point de vue entomologique, l'Arabie me parait devoir être réunie à l'Afrique. 
catulus CAND. Elat. nouv. IV, p. 44 . . . . Abyssine commotus CAND. Ibid., p. 44 . . . . . . . 》

fabalis CAND. Monogr. $\amalg$, p. 201 . . . . . ”

submaculatus CAND. Élat. nouv. II, p. 35 . . Zanzibar.

depressus CAND. Ibid: . . . . . . . . . ”

velatus CAND. Ibid. . . . . . . . . . . »

castaneus CAND. Ibid. . . . . . . . . »

angularis CAND. Ibid. III, p. 79. . . . . . ”

folliculus CAND. Tbid. . . . . . . . . . »

obsoletus Gerst. Insektenf. V. Zanz. 1871 . . ”

octonotatus CAND Élat. nouv. II, p. 34 . . . ”

Burdoi CAND. Ibid. III, p. 82. . . . . . . Ugogo.

Usagaræ CAND. Ibid. . . . . . . . . . Isagara.

b. Afrique australe (y compris le Mozambique et Madagascar).

tœniatus KL. Monats. Berl. Acad. 1855, p. 647. Mozambique. lateritius $\mathrm{K} \mathbf{L}$. Tbid.

rufescens $\mathrm{K}$. Thid.

vestitus $\mathrm{K}_{\text {L. Tbid. }}$

Bradshawi CAND. Élat. nouv. IV, p. 40 .

》

binotatus Boнемr. Ins. Caffr. 1, 2, p. 400.

histrio Erichs. Monogr., p. 247 .

)

)

suturalis Вонем. Ins. Caffr. 1, 2, p. 401 .

Zambèze.

Cafrerie.

præmorsus Вонем. Ibid.

Cafrerie.

convexicollis BонЕм. Ibid. . . . . . . . ”

carinicollis Bонем. Ibid. . . . . . . . ”

acuminatus CAND. Élat. nouv. III, p. 81 . . ”

Damaræ CAND: Ibid. I, p. 42 . . . . . . . Damara.

chloroticus CAND. Ibid., p. 41 . . . . . . Svakop.

Wahlbergi CAND. Ibid., p. 42 . . . . . . N'Gami.

Stáli CANd. Ibid. I, p. 36 . . . . . . . . Damara.

sanguinicollis ErICHs. Monogr., p. 285 . . . Cap.

sexguttatus THUNв. Nov. Ins. Sp. II, 1784 . ”

quadriguttatus ERICHs., loc. cit. . ... . » 
repandus Erichs. Monogr., p. 290

rhodopus Erichs. Ibid.

colon Erichs. Ibid.

spilotus CAND. Monogr. III, p. 148.

megacephalus CAND. Ibid., p. 153.

ventralis Erichs. Monogr., p. 294.

hottentottus ERICHS. Ibid., 302.

gibbulus Erichs. Ibid.

lentus ERICHS. Ibid.

influxus CAND. Monogr. III, p. 177.

castanopterus CAND. Ibid., 182

ana 3 CAND. Ibid., p. 179.

Madagascar.

staurophorus CaND. Ibid., p. 143 .

))

junceus Erichs. Monogr., p. 305

fuscatus Erichs. Ibid., p. 301

)

fuscovittatus FaIrMr. Ann. Fr., 1871, p. 42.

ligneus CAND. Élat. nouv. ПI, p. 82

guttifer CAND. Ibid. IV, p. 41.

c. Afrique occidentale.

fulvicornis Erichs. Wiegm. Arch., 1843, p. 225.

Angola.

segnis CAND. Monogr. III, p. 180

)

assessor CAND. Élat. nouv. IV, p. 41 .

Congo.

scutellaris CAND. Ibid., p. 42.

hilaris CAND. Ibid.

jocularius CAND. Tbid.

fulvescens QuEDENF. Berl. Zeitschr., 1886 .

castaneipennis QUEDENE. Ibid.

gilvipennis QUEDENF. Ibid.

aspalax CAND. Monogr. III, p. 175.

Gabon.

moratus CAND. Ibid., p. 172

)

accensus CAND. Tbid., p. 17.8

Calabar.

benibiensis Beauv. Ins. Afr. et Am., p. 11 . . Benin.

conicipennis CAND. Monogr. WI, p. 201 : .

Guinée.

fallax CAND. Ibid,, p. 196 
sparvarius CAND. Monogr. III, p. 174

subspinosus Beauv. Ins. Afr. et Amér., p. 78

Sénégal.

equinus CAND. Monogr. III, p. 200.

)

lætus CAND. Ibid., p. 148

quadriplagiatus ERICHs. Monogr., p. 291 .

phæopterus CAND. Monogr. III, p. 150

discipennis CAND. Tbid. . . . . . . . . ”

languidus CAND. Ibid., p. 174. . . . . . ”

hoploderus Cand. Ibid., p. 175 . . . . . . ”

princeps CAND. Ibid., p. 176 . . . . . . . ”

holosericeus CAND. Ibid., p. 178 . . . . . ”

fastidiosus KLUG, Herm. Atl., 1835, p. 31 . . ”

cognatus Erichs. Monogr., p. 303. . . . . »

instrenuus CAND. Monogr. III, p. 199. . . . »

Mioni CAND. Ibid., p. 200 . . . . . . . . ”

\section{ae Groupe.}

Nouveau continent.

amplicollis Мотsch. Bull. Mosc., 1859, IV, p. 385.

fenestratus Lec. Proc. Ac. Nat. Sc., 1859, p. 86.

Californie.

Lorquini CAND. Monogr. III.

cardisce $\mathrm{S}_{\mathrm{AT}}$, Trans. Am. phil. Soc. IV,p.169. Etats-Unis. convexus Erichs. Monogr.

Dejeani Lec. Trans. Am. Soc. X.

bifasciatus F. Buanch. Tr. ent. Soc. Am., 1889. Californie. coxalis F. Blanch. Tbid. . . . . . . . . Orégon. stigmaticus CAND. Col. Hefte V, 1869, p. 122. Californie. signatus CAND. Élat. nouv. I, p. 40.

pullus F. BLANCH., loc. cit.

Colorado. gemmifer F. Blanch. Ibid. Californie.

tumidicollis Lec. Trans. phil. Soc. X, p. 498 .

abbreviatus F. Blanch. Trans. Am. entom. 
erythropus Errons. Monogr., p. 293 . . . . Caroline N. saturninus LEc, loc. cit., p. 497.

angustatus F. BLANch., loc. cit.

Floride.

convesus SAx, Journ. Acad. Nat. Sc. III,

p. 169.

États-Unis.

amictus Melsh. Proc. Ac. II, p. 158.

erythropus LEC., loc. cit., p. 497.

insulsus CAND. Monogr. III, p. 169

Californie.

Floridæ CAND. Élat. nouv. I, p. 41 . . . . . Floride.

longior Lec. Proc. Acad. Nat. Sc., 1861 . . . Colorado.

nevadensis F. Blanch., loc. cit.

Névada.

gagathes ErioHs. Monogr., p. 297 . . . . . Etats-Unis.

lavicollis Dej. Erichs., loc. cit.

togatus HorN, Tr. entom. Soc. II, p. 301 .

Texas.

Edwardsi HorN, Tbid.

Californie.

mimeticus Hors, Ibid. IV.

latiusculus EscH. Thon Arch. II, p. 34

)

tenebrosus Lec. Trans. Am. ph. Soc. X, p.498. Etats-Unis

de l'Ouest.

fulvipes LEc. Proc., 1859.

convexulus LEc., loc. cit. . . . . . . . Canada.

crinitus F. Blanch. Tr. Am. entom. Snc., 1889. Californie.

pubescens F. Buanch. Ibid. . . . . Colorado.

carbonatus F. Blanch. Ibid. . . . . . . Califoritie.

obscurus Lec. Tr. Am. phil. Soc. X, p. 498 . ”

æneus Horn, Tr. Am, entom. Soc. III, p. $301 . \quad$ ”

robustus Lec., loc. cit. . . . . . . . Texas.

loviçollis LEc. Ibid.

seniculus F. Blanch. Tr. Am. ent. Soc., 1889. Californie.

dispar F. BLANCH. Tbid. . . . . . . . . ”

aptopoides CAND. Élat. nouv. I, p. 40. . . . Mexique.

humeralis FAIRM. Col. Chil., 1860 . . . . . Chili.

bitactus CAND. (Horistonotus) Élat. nouv. II, p. 39.

elegans? Sor. Hist. Chil. 


\section{Se Groupe.}

Australie (').

venustus CAND. Monogr. II, p. 143 . . . . Swan River.

australis CAND. Ibid., p. $269^{\prime}$ (Horistonotus) - Sidney. elisus CAND. Élat. nouv. I (id.), p. 44 . . . . Victoria. lenis CAND. Tbid. (id.). . . . . . . . . . ”

xanthomus CAND. Ibid. (id.), p. 45. . . . . "

divisus CAND. Tbid. (id.). . . . . . . . . ”

humilis CAND. Ibid. (id.) .. . . . . . . ”

fulvosignatus CAND. Ibid. II, p. 36 . . . . Sidney.

bicolor CAND. Tbid. . . . . . . . . . . ”

hamatus CAND. Ibid., p. 37 . . . . . . ”

pallidipennis CAND. Ibid. . . . . . . ”

consputus CAND. Ibid. . . . . . . . Victoria.

octavus CAND. Ibid. . . . . . . . . Swan River.

flavipennis CAND. Tbid. . . . . . . . . ”

minimus CAND. Tbid. . . . . . . . . . ”

compactus CAND. Tbid. II, p. 83 . . . . . Melbourne.

despectus CAND. Mbid. . . . . . . . . . ”

consobrinus CAND. Ann. Gênes, 1878, p. 133. Somerset. longicornis CAND. Ibid. (Horistonotus) . . . ”

Mastersi M. LeAY, Tr. N. S. W. II, p. 258 . Queensland.

\section{Cardiotarsus.}

Eschs. Silberm. Rev. entom. VI, 1836.

Cardiophorites à quatrième article des tarses dilaté.

acuminatus Goḱn. Voy. Lefebr., p. 284. . Afrique orient. byssinus Bонем. Ins. Caffr. 1, 2, p. 396.

(1) Les Cardiophorus australiens ont le corselet souvent construit comme les Horistonotus. Ils forment le passage des uns aux autres. J'ai donné (Élat, nouv. II, p. 36) les raisons de leur réunion aux Cardiophorus. 
dorsalis Erichs. Germ. Zeitschr. II, p. 333.

Cap . grisescens Farnm. Ann. Fr., 1871, p. 40 . . . Madagascar. silaceus BoHerr. Ins. Caffr. 1, 2, p. 394 . . . Cafrerie. capensis CAND. Monogr. ШI, p. 226. . . . . Cap. philautus CAND. Ibid. . . . . . . . . » crocipes CAND. Elat. nouv. I, p. 43. . . . . ” vitellinus Kuvg, Ins. Madag., 1832, p. 67 . . Madagascar. tabidus Ericis., Cand. Monogr.

bivittatus Böeм. Ins. Caffr. 1, 2, p. 404. . . Natal. silaceus Bонем. Ibid. . . . . . . . . . Gariep. brunneicollis Erichs. Germ. Zeits. II, p. 334. Madagascar. punctatus CAND. Élat. nouv. IV, p. 451 . . . ” scriptus CAND. Ibid. . . . . . . . . ” vulneratus CAND. Monogr. III, p. 229. . . . Ceylan. sinensis CAND. Ibid., p. 230 . . . . . . . Hong Kong. ciliatus CAND. Élat. nouv. I, p. 43 . . . . Bornéo.

Aptopus.

Eschs. Thon Arch., 1829, II, I, p. 32.

Crochets des tarses pectinés.

pruinosus Erichs. Germ. Zeitschr. II, p. 329 . Mexique. venator Dej. CAT.

spadiceus ERICHS. Tbid. . . . . . . . . . ”

lateralis Erichs. Ibid. . . . . . . . . ”

basalis Erichs, loc. cit.

concolor ERICHs. Ibid.

brevis CAND. Elat. nouv. IV, p. 45 . . . . . ,

campylinus Erichs, loc. cit. . . . . . ”

var. decumanus ERICHs., loc. cit.

var. linearis EricHs., loc, cit.

peregrinus Honn, Am. Tr. ent.1884 . . . . Texas.

agrestis Erichs. Germ. Zeitschr. II, p. 331. . Brésil.

Pichinchæ CAND. Élat. nouv. IV, p. 45 . . Équateur. 


\section{TRIPLONYCHUS.}

CAND. Monogr. III, 1860, p. 236.

Crochets des tarses tricuspidés.

longicollis ErICHs. Germ. Zeitschr. II, p. 326. acuminatus CAND. Monogr. II, p. 238 . . . plagiatus ERICHS., loc. cit. Bahia. ventralis CAND. Monogr. II Cayenne. Para. ephippiger Escrs. Thon Arch. II, I, p. 32 . . bisignatus ErICHs., loc. cit. cayennensis Erichs., loc. cit.

Cayenne. rufus CAND. Monogr. III, p. '241 . Lebasi CAND. Ibid.

Cayenne. Brésil. costatus CAND. Ibid. fulvus CAND. Élat. nouv. I, p. 44. cingulatus CAND. Ibid.

Carthagène.

Para. debilis Erichs. Germ. Zeitschr. II, p. 340 . . Colombie. amabilis Krasch, Berl. Zeits., 1866, p. 183 . . Bogota.

HoRIsTONOTUS.

CAND. Monogr. IH, 1860, p. 244.

Cardiophorus Er.

Prothorax limité de chaque côté par une arête s'étendant jusqu'au sommet; tarses non dilatés ni lamellés. Américains.

\section{1 er Groupe.}

Espèces des États-Unis.

vulneratus HoRn, Tr. Am. entom. Soc. 1884, p. 35 . Arizona. pullatus Hons, Ibid. ) densus Lec. New. Spec, 1863, p. 83. . . . . Californie. incanus Lec. Tr. Am. phil. Soc. X, p. 499 . . ” basalis Horn, Tr. Am. ent. Soc., 1884 . . . ” transfugus Lec. (Card.), loc. cit. . . . . ” 
definitus HoRN, loc. cit. sufflatus LEC. (Card.), loc. cit.

Californie.

simplex LEC. New. Sp., 1863, p. 83

curiatus SAY, Trans. Am. phil. Soc. VI, p. 173.

)

areolatus ErIchs. Zeitschr. II, p. 320.

gracilis Horn, Trans. Am. ent. Soc., 1884.

Névada.

exoletus Erichs. Zeits. f. d. Ent. II, p. 325 .

Texas.

Uhleri HonN, loc. cit.

Georgie.

mitis Hons, Ibid.

Utah.

$2^{e}$ Groupe.

Mexique, Antilles, Amérique centrale.

brunneus CAND. Élat. nouv. IV, p. 45.

Mexique.

crux-nigra Chevr. Ann. Fr., 1867, p. 610

Cuba.

asthenicus CAND. Monogr. III, p. 266.

Haïti.

bignogniæ CAND. Ibid.

badius CAND. Ibid., p. 260

))

Cuba. rufiventris CAND. Élat. nouv. III, p. 84 . . . Guatémala. pedestris CAND. Ibid.

\section{Groupe.}

Amérique du Sud.

spernendus ERICHs. Zeitschr. II, p. 320.

Vénézuéla.

attenuatus Erichs. Ibid., p. 325.

»

dioptricus Erichs. Ibid.

acutipennis CAND. Monogr. III, p. 258

)

《e

pubipennis CAND. Ibid., p. 259

)

diplotrix UAND. Ibid., p. 252 .

Para.

distigma CAND. Élat. nouv. II, p. 39

Cayenne.

zonatus CAND. Monogr. III, p. 251 .

Para.

æoloides CAND. Ibid.

basilaris CAND. Élat. nouv. III, p. 84 .

)

)

u mbrosus Erichs. Zeitschr. II, p. 308.

"

biguttatus CAND. Monogr. III, p. 253.

Brésil. 
apicalis GERM. Zeitschr. II, p. 251. . . . .

Brésil.

posticatus ErICHs. Tbid., p. 309. . . . . . ”

effusus Erichs. Tbid., p. 322 . . . . . . . ”

oblitus Erichs. Ibid. . . . . . . . . . ”

femoratus Erichs. Ibid., p. 318 . . . . . ”

ligatus ErICHs. Tbid., p. 324 . . . . . . ”

pubescens CAND. Monogr. III, p. 257. . . . ”

dimidiatus ERICHS. Zeitschr. II, 317 . . . . ”

flavidus CAND. Monogr. III, p. 250. . . . . ”

electus CAND. Ibid. . . . . . . . . . . ”

convexicollis CAND. Ibid., p. 260 . . . . Rio.

parmenoides CAND. Tbid., p. 261 . . . . . Montévideo.

Arechavaletae CAND. Élat. nouv. M, p. 85 . ”

castaneus Stenn. Rép.Argent. XV, p.568 . Pampas.

canescens STEINH. Tbid. . . . . . . . . ”

farinosus CANd. Bull. Soc. zool. Fr. 1889 . Grand-Chaco.

insularis BoнEмr. Eugen. Resa, 1858 . . . . Otaïti.

popularis Erichs. Wiegm. Arch., 1847 . . . Pérou.

rufulus ERICHs. Zeitschr. II, p. 319. . . . . Brésil.

Gleryi Guḱr. Voy. Favor., p. 27 . . . . . . Pérou.

tetraspilotus Guḱr. Ibid. . . . . . . . »

bitactus CAND. Élat. nouv. II, p. 39 . . . . Chili.

crucifer CAND. Monogr. III, p. 264. . . . . Rio.

exiguus Erichs. Zeitschr. П, p. 321 . . . . Colombie.

minimus CAND. Élat. nouv. III, 85 . . . . . Brésil.

humeralis CAND. Monogr. III, p. 267 . . . . ”

melanorhax CAND. Ibid. . . . . . . . »

unitinctus CAND. Tbid. . . . . . . . . »

posthumus Erichs. Zeitschr. II, p. 319 . . . Colombie.

EsTHESOPUS.

Eschs. Thon Arch. I, II, p. 32.

Cardiophorus ERICHS.

Horistonotus, avec le quatrième article des tarses dilaté, lamellé. 
claricollis SAY, Trans. Am. phil. Soc. VI, p. 169 .

Virginie.

præditus Horn, Am. ent. Soc. Tr., 1884.

Texas.

parcus HonN, Ibid.

Arizona.

dispersus Hons, Tbid.

))

pusio Horn, Ibid.

Floride.

bicolor Hons, Ibid., 1874

)

pædicus CAND. Monogr. III, p. 277

Antilles.

Monocr. unifasciatus DeJ. Cat.

humilis CAND. Tbid., p. 284.

Mexique.

nitidulus CAND. Ibid., p. 279 .

)

atripennis CAND. Ibid., p. 280

))

apicatus CAND. Élat. nouv. III, p. 85 . . . . »

murinus CAND. Monogr. III, p. 284

quadrivulneratus Erichs. Zeitschr. II, p. 339. N. Grenade. quadripustulatus CAND. Monogr. III, p. 285. Vénézuéla. troglodytes Gerir. Zeitschr. I, p. 232.

)

infimus ERICHs. Zeitschr. Ш, p. 337.

Colombie.

inconspicuus ERICHs. Tbid.

)

morio CAND. Élat. nouv. II, p. 39. . . . . . Équateur.

hepaticus EnICHs. Zeitschr. II, p. 336 . . . Guyanne,

Colombie, Vénézuéla.

bellus CAND. Élat. nouv. III, p. 85 . . . . . Eqa.

carbonarius CAND. Monogr. III, p. 282 . . . Cayenne.

Glarkii CAND. Tbid., p. 276. . . . . . . Brésil.

delinitor CAND. Ibid. . . . . . . . . ”

placidus Erichs. Zeitschr. II, p. 335 . . . . »

physalus CAND. Monogr. III, p. 278 . . . . ”

curtus Cand. Tbid., p. 279 . . . . . . . . ”

prœcox CAND. Ibid. . . . . . . . . ”

castaneus Eschs. Thon Arch. II, I, p. 32 . . ”

sordidus ERICHs. Zeitschr.

angusticollis CAND. Monogr. III, p. 281 . .

humeralis Steinh. Att. Soc. Ital. XV . . .

Plata. 


\section{TRIBU XIV.}

\section{MÉLANOTITES.}

Front rebordé; crochets pectinés; écusson non cordiforme.

Pselliss.

CAND. Monogr. III, p. 289.

Troisième article des tarses dilaté.

promiscua ERICHs. Zeitschr. f. d. Entom. III,

p.112. . . . . . . . . I. Mascareignes.

\section{Diploconus.}

Cand. Monogr. III, p. 290.

Melanotus, Cratonichus auctor.

Sutures prosternales non creusées au sommet; hanches postérieures anguleuses au milieu.

frontalis CAST. Hist. Nat. Ins. I, p. 251 . . . Malaisie. porrectus ERICHS. Zeitschr. III.

prominens ERICHs. Germ. Zeitschr. III, p. $109 . \quad J a v a$. peregrinus CAND. Monogr. III, p. 293 . . . Bornéo. exquisitus CAND. Ibid., p. 294 . . . . . . . ” homostictus CAND. Ibid., p. 295. . . . . . ” nigripennis CAND. Élat. nouv. III, p. 86. . . Sumatra. Hasselti Cand. Notes Leyd. Mus. 1883, p. 205. rufus CAND. Monogr. III, p. 295. . . . . . Java. nigricornis CAND. Ibid., p. 296 . . . . . . . ” plagiatus CAND. Ibid. . . . . . . . . . . ” angulatus CAND. Ibid., p. 297 . . . . . . Hongkong. separandus Krich, Mitt. Mus. zool. Dresd. 1875, p. 8. spiloderus CAND. Élat. nouv. I, p. 45 . . . Malacca. coracinus CAND. Monogr. III, p. 494 . . . . Indo-Chine.

Var. melanopterus CAND. Élat. nouv. I; p. 46. 
ambustus CAND. Élat. nouv. I, p. 46 . . . Sumatra. dorsalis CAND. Ibid. II, p. 39. . . . . . . Bornéo. pilosus CAND. Ibid. . . . . . . . . . . Célèbes. ineptus CAND. Ibid. . . . . . . . . . . ” consanguineus CAND. Monogr. III, p. 293 . Malaisie. angusticollis CAND. Ann. Entom. Belg. XVII,

bullet. . . . . . . . . . Philippines. erythronotus CAND. Élat. nouv. I, p. 46. . . » cervinus CAND. Ann. ent. Belg. XVII, bull. . ” ciprinus CAND. Élat. nouv. I, p. 47 . . . . » umbilicatus CAND. Ann. ent. Belg. XVIII, bull. ” politus CAND. Tbid. . . . . . . . . . . ” erythropus CAND. Ibid. . . . . . . . Ternate. ardjoenicus CAND. (sub nom. umbilicatus).

Élat. nouv. III, p. 86 . . . . . . Java. aequalis CAND. (melanotus). Ann. Mus. Gênes, XII, 1878, p. 136. . . . . . . . Nour. Guinée. barbus CAND. Élat. nouv. III, p. 87 . . . Célèbes.

\section{Melanotus.}

Eschs, in THoN, Arch. I, II, p. 32.

Cratonychus ERIChs., Le Conte, etc. Perimecus StePh. Ctenonychus MeLsh. Priopus Cast.

Des sillons basilaires latéraux au prothorax; hanches postérieures non anguleuses.

\section{Ier Groupe.}

Espèces de l'ancien continent et iles dépendantes.

punctolineatus PeLERIN, Vig.Zool. Journ. IV, p. 524. . . . . . . . . . . . Enrope. - niger FABR. (error) in CAND. Monogr.

ater Eschs. Th. Arch.

aterrimus Or. Ent. 
tenebrosus Errchs. Germ. Zeitschr. III, p. 93. Eırope mér. brunnipes Germ. Ins. Sp. nov., p. 41 . . . . ” fascicularis Küst. Käf, Eur. subvestitus BruLli, Exp. Mor.

monticola MÉnÉTr. Cat. Rais., p. 156 . . . . Talische. brunnipes Kor. Melet.

sulcicollis Muls. Opusc. entom. VI, p. 19 . . Pyrénées. cuneiformis BAUDr, Berlin. Ent. Zeitschr. 1871, p. 53 . . . . . . . . . . . . . . Chypre. sobrinus Mḱnétr. Cat. Rais. 1832, p.157 . . Caucase. Menetriesi Fadid. Nouv. Mém. Mosc. IV. castanipes PAYK. Fn. suec. III, p. 23. fulvipes GruL. Ins. suec. . . . . . Sibérie, Etats-Unis. incequalis Lec. Tr. Am. phil. soc. X. scrobicollis LEC. Ibid. longipennis Küsт. Käf. Eur. anachoreta MÉnére. Cat. rais., p. 159 . . . Talische. rufipes Herbst, Fuessi. Arch. V, p. 113. . . Europe. atripennis Eschs. Mém. Mosc. VI. brunnipes Lac. Faun. Par. erythropus Gmex. Ed. Linn. 1, IV. fulvipes HerBst, Käf. rugosus MARSH. Entom. brit. Var. bicolor FABR. System. Eleuth. II. crassicollis Erichs. Germ. Zeitschr. III,p. 98. Europe centr. tristis Küsт. Käf. Eur.

picticornis Heyd. Entom. Reis. Span. in Berl. Zeitschr.1870, p. 19. . . . . . . . . Espagne. Hidalgoi Perez, Ann. Soc. Hisp. Hist. nat. 1874. punctatocollis Brisout, Ann. Fr. 1861, p. 600. Hyères. Candezei Stierl. Berl. Ent. Zeitschr. 1864, p. 149.

Desbrochersi CAND. Élat. nouv. II, p. 87 .

Sicile. bernhardinus Stierin, Mith. Schw. Ent. Ges. 1879, p. 439. . ... . . . . Suisse. 
dichrous Erichs. Germ. Zeitschr. III, p. 93. . Algérie. amplithorax Murs. Opusc. VI.

bicolor GxLL. in Schönh. Syn. Ins.

avitus CAND. Deutsch. Ent. Zeitschr. 1886, p. 186.

morbosus CAND. Tbid. compactus Cand. Monogr. III, p. 317.

Alaï. mauritanicus Loc. Expl. Alger., p. 162 . . . robustus Erichs. Germ. Zeitschr. III, p. 99. . Asiemineure. fusciceps GyLL. Schönh. synon. App., p.135 . Turquie. æmulus ERICHs. Zeitschr. III, p. 97 . . . . Grèce. bajulus ErioHs. Ibid. . . . . . . . . . syrie. torosus Erichs. Ibid. . . . . . . . . . ” mutatus Har. Coleopt. Hefte V, 1889 . . . Perse. persicus Fald. N. Mém. Mosc. 1835. persicus MÉnÉtr. Cat. rais., p. 157 cete CAND. Monogr. III, p. 332 Japon. amussitatus CAnd. Mém. Soc. Sc. Liége V, $2^{e}$ sér. invectitius CAND. Élat. nouv. I, p. 47. restrictus CAND. Ibid., p. 47 . annosus CAND. Ibid., p. 48. senilis CAND. Ibid., p. 47 laticollis Mots. Etude entom. 1860 Algérie. ) legatus CAND. Monogr. III, p. 323 . correctus CAND. Élat. nouv. I, p. 48 spernendus Cand. Mém. Soc. Sc.Liége V, $2^{\circ}$ sér. seniculus CAND. Ibid.

Fortnumi CAND. Élat. nouv. II, p. 40 . erythropygus Cand. Mém. Liége $\nabla, 2^{e}$ sér. caudex Lewis, Ent. Montl. Mag. déc. 1879 , propexus CAND. Monogr. III, p. 326 Chine. nuceus CAND. Élat. nouv. III, p. 89 . venalis CAND. Monogr. III, p. 323 . 
regalis CAND. Ibid.

Chine.

sciurus CAND. Élat. nouv. III, p. 89.

)

ventralis CAND. Monogr. III, p. 324 . . . . ”

carbonarius CAND. Élat. nouv. III, p. 88 . . ”

arctus CAND. Tbid., p. 89 . . . . . . . . ”

canaliculatus FALD. Mém. Acad. Pét. II,

p. 362.

piger Mотscr. Etudes entom. 1853, p. 45

umber Bates, Proc. Zool. Soc. 1866, p. 348 .

tamshuinensis BATES, Tbid.

longicornis CAND. Monogr. III, p. 332 . . . Inde bor.

labidus ERICHs. Germ. Zeitschr. III, p. 111.

vafer ERICHs. Tbid.

Bengale.

egens CAND. Monogr. III, p. 321 .

)

)

incallidus CAND. Élat. nouv. III, p. 88

Annam.

nitidus CAND. Monogr. III, p. 334

Inde or.

fuscus FABR. Syst. Eleuth. II, p. 228 .

))

hirticornis Heribst, Käf. X.

orientalis Des. Cat.

vermiculatus CAND. Monogr. III, p. 336

Ceylan.

sulcatus CAND. Tbid.

Inde bor.

tomentosus CAND. Ibid.

)

albivellus CAND. Élat. nouv. I, p. 48 .

Java.

badiipennis CAND. Monogr. III, p. 334 . . . Inde or.

brevis CAND. Élat. nouv. III, p. 88 . . . . : Cochinchine.

hæmorrhous CAND. Monogr. III, p. 333. . . Inde or.

apicalis Fledt. Ann. Fr., 1889, p. 144 . . . Camboge.

gobius CAND. Monogr. ПI, p. 329 . . . . . Java.

veles CAND. Ibid. . . . . . . . . . »

rusticus ERICHs. Germ. Zeitschr. III, p. 107 . ”

bipunctatus CAND. Élat. nouv. I, p. 49 . . . ”

cuneolus CAND. Monogr. III, p. 329 . . . . »

promtus ERIchs., loc. cit. . . . . . . »

rubidus Erichs. Tbid. . . . . . . . . ” 
puberulus ERICHs. Ibid. Java. porcellus CAND. Ann. Mus. Gênes XII, p. 136. )) hirtellus CAND. Monogr. III, p. 330. . . . . insularis CAXX. Élat. nouv. IV, p. 46. . . I. Andamans. hapatesus CAND. Notes fr. Leyd. Mus., 1880. Sumatra. carinatus CAND. Élat. nouv. I, p. 49 . . . . ” brevicornis CAND. Ann. Mus. Gênes, 1880, p. 195. cribrum CAND. Ibid. ruficaudis CAND. Tbid.

pisciculus CAND. Monogr. III, p. 330. ) ) phlogosus CAND. Ibid. ebeninus CAND. Ibid.

Albertisi CAND. Ann. Mus. Gênes XII, p. 135. N. Guinée. potensis Montr. Ann. Fr., 1860, p. 257 . Nouv. Calédonie. agriotides CAND. Monogr. III, p.322. . . . Sénégal. umbilicatus GrLL. in Schönh. Syn. Ins. Afrique intertrop. madagascariensis CAND. Elat. nouv. IV, p. 46. Madagascar.

\section{a Groupe.}

Espèces américaines.

corticinus SAY, Journ. Acad. Phil. III, p. 123. Georgie. longulus Lec. Trans. Am. phil. Soc. X, p. 473. macer LEC. Ibid. Californie. decumanus Erichs. Germ. Zeitschr. III, p.104. Maryland. cuneatus LEc., ]oc. cit.

incertus LEC. Tbid.

canadensis CAND. Monogr. ПI, p. 342. . . . Canada. despectus CAND. Ibid., p. 343 . . . . . . Georgie. clandestinus Erichs. Germ. Zeitschr. III, p. 112. secretus Lec. Trans. Am. phil. Soc. X, p. 434. ignobilis Melsh. Proc. Ac. Phil. II, p.152 . . Pensyleanie. depressus MeLsh. Ibid.

Etats-Unis.

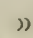


angustatus ErICHs. Germ. Zeitschr. III, p.113. Pensylvanie. testaceus MeLsh., loc. cit.

trapezoideus LEc. Trans. Am. phil. Soc. X, p. 475 .

tænicollis LEC. Ibid.

Leonardi Lec. Tbid.

texanus CAND. Monogr. III, p. 351.

Texas.

glandicolor Mess. Proc. Ac. Phil. II, p.152 . Etats-Unis

communis GyLL. Schönh. Syn. Ins. 1, 3, App.,

p. 138.

cinereus WEB. Obs. Ent. p. 77 (pars).

simplex Germ. Ins. Sp. nov., p. 42.

spadix ERICHs. Germ. Zeitschr. III.

fissilis SAY, Trans. Am. ph. Soc. VI, p. 183. .

)

brevicollis HeRBSt, Kaf?

cinereus WEB., loc. cit. (pars).

laticollis ERICHS., loc. cit.

ochraceipennis, sphenoidalis MeLSH., loc. cit.

exuberans Lec. Trans. Am. phil.Soc. X, p.477. Californe. parumpunctatus Melsh. Proc. Ac. Phil. II,

p.151. . . . . . . . . . Pensylvanie.

effetus CAND. Monogr. III, p. 355 . . . . . Ohio.

verberans Lec. Trans. Am.phil.Soc.X, p.478. Maryland. emissus Lec. Ibid. . . . . . . . . . Georgie.

infaustus LEc. Tbid.

cribulosus Lec. Ibid. . . . . . . . . Nebraska. cribricollis CAND. Monogr. III, p. 358 . . . Etats-Unis.

paganus CAND. Tbid. . . . . . . . . . ”

pertinax $S_{A Y}$, Trans. Am. phil. Soc. VI, p. 185.

dubius Lec. Ibid. X, p.479. . . . . . . . New-York. tenax SAY, Ibid. VI, p. 185 . . . . . . . États-Unis.

americanus Henbst, Käf. X, p. 74 . . . . ”

insipiens SAy, Trans. Am. phil. Soc. VI, p. $184 . \quad$ ”

tenellus Errohs., loc. cit., p. 114. 
gradatus Lec. Proc. Acad. Phil., 1866, p. 390. Maryland. opacicollis LEc. Ibid. . . . . . . . . . Illinois. variolatus LEC. Ibid., 1861, p. 347. . . . . Californie. oregonensis LEC. Trans. Am. phil. Soc. X, p.480. . . . . . . . . . Orégon. morosus CAND. Monogr. III, p. 346 . . . . Georgie. longulus LEC., loc. cit., p. 480.

sagittarius Lec. Trans. Am. phil. Soc. X, p. 480 . paradoxus Mersh. Proc. Ac. Philad. $\Pi$, p. 152. Pensylianie. abdominalis Erichs. Germ. Zeitschr. III,

p. $10 t$. vetulus ERICHS. Tbid. États-Unis. similis Kerby, Fn. bor. Amer. IV, p. 149 . . Canada. peninsularis CAND. Élat. nouv. IV, p. 46 . . Floride. prolixus Erichs. Germ. Zeitschr. III, p. 94 . Mexique. Rodriguezi CAxd. Élat. nouv. II, p. 40 . . Guatémala. rubicundus CAND. Ibid. IV, p. 46

\section{TRIBU XV.}

\section{ATHOÏTES.}

Front généralement subquadrangulaire, bordé en avant; écusson non cordiforme; hanches postérieures étroites; tarses simples, parfois les $2^{\theta}$ et $3^{\theta}$ élargis; ongles non pectinés.

\section{LIMONIUS.}

Eschs. Thon, Arch. II, I, p. 33.

Gambrinus Lec. Pheletes Kresenw.

Premier article des tarses égal au suivant ou à peine plus grand.

\section{(er Groupe.}

Ancien continent.

rufus CAND. Monogr. III, p. 384 . 
violaceus MürL. in Germ. Mag. IV, p. 184 . . suturalis GEBL. Bull. Acad. Petr. III, p. 1845, p. 99 .

Europe.

Kraatzi CAND. Deutsch. Entom. Zeits., 1879, p. 281

pilosus Leske, Reise Sachs., 1785, p. 11.

nigripes GruL., CAND. Monogr. III.

cylindricus Rossi, Mant. Ins.

villiger Solski, Hor. Soc. Ross., 1882, p. 237.

æruginosus Outv. Entom. II, 31, p. 33

Tashkend.

cylindricus PAYK., CAND. Monogr. III.

obsoletus Marsh. Ent. Brit. I.

nigripes STEPH. Illustr. Brit.

parallelus Mots. Schr. Reis., 1860, p. 111 . . turdus CaND. Monogr. III, p. 393 .

Sibérie. minutus LinN. Syst. Nat. I, 2, p. 656

France mér.

angustus HeRBst, Käf. X.

crenulatus FALD. Fn. Transc. I.

2 forticornis $\mathrm{BACH}$, Kaferf. III.

nigroceneus MARSH. Ent. Brit. I.

serraticornis. STEPH. Ill. Brit. III.

var. cereus Bruldi், Exp. Mor.

var. nitidicollis CAST. Hist. Nat.

parvulus Pz. Faun. Germ., p. 61

mus Ixlig. Magaz. VI, p. 12.

lythrodes Gerir. Mag. Entom. I, 1813, p. 129.

Europe.

marginellus PerRIS, Ann. Fr., 1864, p. 264

.)

æneoniger de GeER, Mém. Ins. IV, 1774, p. 159.

Madrid.

Bructeri Panz. Ent. Tasch., 1795, Cand. Mon.

Europe.

minutus $\mathrm{P}_{\mathrm{AYK}}$

nitidus Grex. Ed. Linn. 1, 4.

substriatus GeBL. Bull. Mosc. VI, 1833, p. 279.

Sibérie.

araratensis FALD. Nouv. Mém. Mosc. IV,

p. 370 .

Ararat. 
Baconi CAND. Monogr. III, p. 412 Himalaya. vittatus CAxd. Mém. Soc. Sc. Liége $V, 2^{\text {e }}$ sér. atricolor Lewis, Ent. Monthl. Mag. 1879, p. 155 .

\section{2e Groupe.}

Nouveau continent.

auriplis $\mathrm{S}_{\mathrm{AT}}$, Journ. Ac. Phil. II, p. 172 . Artiansas. pubicollis Lec. Trans. Am. phil. Soc. X, p. 429. fulvipilis $\mathrm{C}_{\mathrm{AND}}$. Monogr. III, p. 383 Californie. mirus Lec. Trans. Am. phil. Soc. X, p. 429. Ulkei Hons, Tr. Am. ent. Soc. 1871.

Grotchi Hons, Ibid., 1873 discoideus LEc. Proc. Ac. Phil. 1861, p. 348 a urifer LEc. Trans. Am. phil. Soc. X, p. 429 stigma Herbst, Käf. X, p. 86 armus $\mathrm{S}_{\mathrm{AY}}, \mathrm{Tr}$. Am. phil. VI.

griseus Beauv. Ins. Afr. et Am., p. 214 . . Japon. )) cylindriformis SAX, Journ. Ac. Phil. III. hirticallis MeLsh. Proc. Ac. phil. II. confusus Lec. Trans. Am. phil. Soc. X, p. 430. ænescens Lec, Ibid. . . . . . . . . New Jersey. plebejus SAx, Ann. Lyc. I, p. 263 . . . . . Etats-Unis. infuscatus Mотs. Bull. Mose. 1859, p. 371 . Orégon. M. Rocheux. Etats-Unis. ) $》$ occidentalis CAND. Monogr. III, p. 411 . . . æger Lec. Trans. Am. phil. Soc. X, p. 431 . Lac Supérieur. pectoralis Lec. Proc. Ac. Phil. 1866, p. 391. . Californie. quercinus $\mathrm{S}_{\triangle \mathrm{Y}}, \mathrm{Ann}$. Lyc. I, p. 262. . . . . Etats-Unis. maculicollis Мотsсн. Bull. Mosc. 1869, IV, p. 372. Californie. discicollis CAND. Élat. nouv. I, p. 49. ornatulus Lec. Report Surv. 1857, I, p. 46. ) humeralis CaNd. Monogr.

basillaris $\mathrm{S}_{A Y}$, Journ. Ac. Phil. III, p. 172 . . Missouri. semiceneus LEC. Trans. Ac. phil. Soc. X. 
nitidicollis Lec. Ann. a. Mag. II, p. 378 . . . Vancouver. consimilis WALKER, Nat. Vancouver.

nitidulus Hors, Tr. Am. Ent. Soc. 1871, p. 315. Orégon. subauratus Lec. Trans. Am. phil. Soc. X, p. $432 . \quad$ » pilosulus Lec., Ibid. (pour pilosus) . . . . Californie. californicus Man. Bull. Mosc. 1843, II, p. 238. cribricollis Hons, Trans. Am. Ent. Soc. 1871, p. 314 . . . . . . . . . . . . . »

canus Lec. Trans. Am. phil. Soc. X, p. 433. . j propexus CAND., Monogr. III, p. 386 . . . Nouv. Orléans. anceps Lec., loc. cit. . . . . . . . . . Etats-Unis. €ctipus SAy, Tr. Am. phil. Soc. VI, p. 167 . . Pensylvanie. agonus SAY, Ibid.

ornatipennis LeC. New. Sp. Col. I, 1863, p. 84. definitus Ziegl. Proc. Acad. Phil. II, p. 268. nimbatus SAY, Ann. Lyc. I, p. 255. infernus Lec. Tr. Am. phil. X.

quadraticollis CAND. Monogr. III, p.412 . Mexique.

Caroline. New-York.

Elathous.

Reitter, Entomol. Nachr., 1890, p. 247.

Voisin du g. Isidus. Intermédiaire entre les Elater et les Athous.

Buyssoni Reitr., loc. cit. . . . . . . . Arménie. Candezei Rertr. Ibid. . . . . . . . . Crimée.

Ismos.

REX, Ann. Soc. Linn. Lyon XXI, 1874, p. 405.

Premier article des tarses allongé aux quatre pattes postérieures.

Moreli ReY, loc. cit.

Corse.

Pitrobios.

Lec. Trans. Am. phil. Soc. X, p. 428.

Antennes des mâles bipectinées; de courtes lamelles aux tarses. 
anguinus Lec., loc. cit. . . . . Etats-Unis, Canada.

Billingsi Bland, Proceed. Ent. Soc. Phil. 1864, p. 69.

Murrayi Lec. Proc. Acad. Philad. 1860, p. 347. Culifornie.

\section{AтHобs.}

Eschs. Thon, Arch. II, I, p. 33.

Anathotus Steph., Crepidophorus Muls., Eschscholtzir CASt., Pedetes Krrby, Stenagostus, Grypocarus Trows. Harminius FATRM.

Front carré, concave, rebordé en avant; hanches postérieures étroites ; tarses à articles simples, le premier allongé, 2 et 3 parfois un peu élargis; élytres ordinairement plus larges que le prothorax; coloration habituellement brune; sexes souvent dissemblables.

\section{Ier Groupe.}

Ancien continent.

rufus DE GEER, Ins. IV, p. 144 . . . . . . Allemagne. melanophthalmus Fourcr. Ent. par., p. 39. testaceus FвӧнL. Naturf., p. 26.

rhombeus OL. Entom. II, 31, p. 22 . . . . Europe. pubescens StePH. Illustr. brit. III, p. 274.

robustus Strerl. Mittheil. Schweiz. Ges. 1863, p. 91 .

p. 373... . . . . . . . . Autriche. niger Lrsw. Syst. Nat. I. II, p.656. . . . Europe. aterrimus FABR. Syst. Eleuth. hirtus Herbst, Füessl. Arch. deflexus, porrectus. THous. Skand. Col. var. scrutator HERBST, Käf. † alpinus Redr. Faun. Austr. semirufus, aneithorax. Dessa. Abeille VII. loesus GERM. Ins. Sp. nov.

nigritulus Reitr. Entom. Nachricht. 1890, p. 244. Caucase. vulpeculus ReIrT. Tbid. 
oblongus Solsky, Horæ entom. Ross. 1871. . Nladivostock. tartarus CAND. Monogr. III, p. 426 . . . . Crimée. mutilatus RosenH. Beitr. I, 1847, p. 16 . . Allemagne. foveolatus HAMPE, Stett. Zeit., 1850.

anthracinus Muls. Mém. Ac. Lyon 1854.

cavatus CAND. Monogr. II, p. 43 . . . . Batoum.

Menetriesi Reiтt. Entom. Nachr.1890, p. 243. M. Talysch.

Fausti Reitt. Ibid. . . . . . . . . . . Cancase. jocosus CAND. Élat. nouv. II, p. 90. . . . . Amur. lætus CAND. Deuts. Ent. Zeits., 1879, p. 281. . ” hæmorrhoidalis FABR. Syst. Eleut. II, p. 235. Europe. dimidiatus Drapiez, Ann. gén. sc. phys., 1820. leucophreatus LAc. Faun. dar. ruficaudis GriL. Ins. suec. obscurus PaYK. Fn. suec. analis HERBST, Käf.

algirinus CAND. Monogr. III, p. 451 . . . . Algérie. Raffrayi Desbr. Abeille IX.

corticeus FAIRM. Ann. Fr. 1870, p. 380 . . . Maroc. limoniformis CAND. Élat. nouv. I, p. 50 . . . Toscane. parallelopipedus BrulLí, Exp. Mor. III, p.139. Grèce. dasycerus Burss. Ann. Soc. Fr. Bull. oct. 1890. Turquie. obsoletus ILLIG. Mag. VI, p. 2 . . . . . . Portugal. rubrotestaceus Desbr. Mitt. Schw. Ent.

Ges. III. . : . . . . . . . . . . . Andalousie. difficilis Dufour, Bull. Soc. Pau, 1843, p. 41. Pyrénées. mingrelicus ReIтт. Entom. Nachr. 1890, p. 247. Mingrélie. longicollis OL. Ent. II, 31, p. 38. . . . . . . Europe. + crassicollis Lac. Faun. Par., p. 642. unicolor, lateralis MinsH. Ent. brit.

vittatus FABR. Ent. syst. 1, 2, p. 224. murinus Reiche, Ann. Soc. Fr. 1869, p. 380. semipallens MuLs. Op. ent. VI. advena Scop. Ent. carn. lineatus VmLens, Ent. I, p. 304. 
conicicollis Desbr. Abeille VII, p. 109 . Fr: mér. debilis Reiche, Ann. Fr. 1889, p. 382 . Espagne sept. nigricornis Bris. Ann. Fr. 1866, p. 377. Madrid. puncticollis KIES. Nat. Ins. IV, 2, p. 316 Fr. mér. cingulatus MrnI. Dentsch. Ent. Zeitschr. 1881, p. 325

ineptus CANd. Monogr. III, p. 442

Cattaro. Sicile. angustulus CAND. Tbid., p. 443 Portugal. cantabricus Schauf. Sitsung Ges. Isis, 1861. subtruncatus Muls. Op. entom. VII, p. 89. Madrid. strictus ReICHe, Cat. Gren., 1863, p. 81. virgatus REICHE, Ibid.

Grandini Dessr. Abeille III. tomentosus Muls. Op. entom. VI, p. 24. propinquus Du Buyss. Ann. Fr., 1889, Bull., p. CCV. sinuatocollis Desbr. Abeille VII, p. 112 crenatostriatus Reiche, Ann. Fr., 1869 villiger MuLs. Op. entom. VI, p. 20. brevicornis Desbr. Mitt. Schw. ent. Ges. III. corsicus + Reiche, Ann. Fr., 1861, p. 207. filicornis CAND. Monogr. II, p. 447 . . . infuscatus Eschs. Thon, Arch. II, I, p. 35 . Sedakovi Mannerh. Bull. Mose., 1852, IV, p. 293. dauricus MannerH. Tbid. Fr. mérid. Turquie. Fr. mérid. ) )

Corse.

Pyrénées. Sibérie. undulatus De GeEr, Ins. IV, p.155 . . . . Europe sept. trifasciatus Henbst, Käf. X.

bifasciatus GrLL. Ins. suec. I.

Sacheri Kres. Nat. Ins. IV, 2, p. 320 .

Bannat. Brucki CANd. Élat. nouv. II, p. 40 Grèce. proximus Haspe, Wien Ent. Monats. VII, p. 190.

† spectabilis HAMPE, Tbid. 
Reynosæ Bris. Ann. Fr., 1866

Madrid.

subfuscus MüLLen, Fn. Fridrichs .

Europe.

analis FABR. Ent. Syst.

marginicollis REITT. Entomol. Nachr., 1890,

p. 245.

Caucase.

daghestanicus Reitт. Ibid., p. 246 . . . . Daghestan.

utschderensis ReITt. Tbid.

Circassie.

cyprius BAUd, Berl. Ent. Zeitschr., 1871, p. 54.

Chypre.

chloroticus CAND. Élat. nouv. I, p. 51 . . . Portugal.

emaciatus CAND. Monogr. III, p. 454. . . . Fr.mérid.

corsicus Reiche, Ann. Fr., 1869, p. 381 (ठ'). . Corse.

cachecticus CAND. Monogr. III, p. 455 . . . Sicile.

Langsdorfi StrenL. Mitt. Schw. Ent. Ges., 1880, p. 290.

Gênes.

crassicornis CAND. Monogr. III, p. 456 . . . Piémont.

Godarti Muls. Opusc. Entom. VI, p. 75 . . . Fr. mér.

flavescens Muls. Mbid. . . . . . . . Savoie.

amicus Perris, Abeille, 1873, p. 4 . . . . . Corse.

tessalonicus ReICHe, Ann. Fr., 1869, p. 386 . Macédoine.

olbiensis Muls. Opusc. Entom. VI, p. 93 . . Fr. mér.

Sena ci Buyss. Ann. Fr., 1889, Bull. ccrv. . . Turquie.

acutangulus FATRM. Ibid., 1866, p. 261 . . Asie mineure.

Zebei BACH, Käf. Faun. II, 1852, p. 32 . . . Allemagne.

biformis REDT. Fn. Austr., p. 493.

'leucophoeus Kres. Nat. Ins. IV, p. 321.

recticollis Graells, Mém. Map. Géol., 1858, p. 45. Castille. spiniger CAND. Monogr. III, p. 460. . . . . Italie, Sicile.

castaneus FatrM. Ann. Fr., 1852. (Harminius.)

florentinus Dessr. Abeille VII.

herbigradus MuLs. Opusc. Entom. VI, p. 26 . Fr. mér.

basalis Cand. Monogr. III.

Chamboveti MuLs. Ann. Soc. agr. Lyon, XV.

sylvaticus Muls. Opusc. Entom. IV, p. 23.

)

var. lcevigatus DessR.

loevistriatus DoF. 
obtusifrons Desbr. Mitt. Schw. Ges. III . Alpes. pallens Mous. Opusc. Entom. VI, p. 24

Eur. mér. cavifrons Redt. Fn. Austr., p. 492.

montanus CAND. Monogr. III, p. 463 .

Suisse. lateralis Bris. Ann. Fr., 1866, p. 378 . . . . Castille. jejunus Kres. Nat. Ins. IV, 2, p. 326 . . . . Tyrol. Raddei Fadst. Hor. Soc. Entom. Ross., 1877 . Cancase. astrabadensis FAUST. Ibid. . . . . . ” transcaucasicus StrerL. Mitt. Schw. ent. Ges. IV,p. 474

Tiflis. limbaticollis Mотьсн. Schr. Reis., p. 108 . . Siliérie. Schneideri Kres. Verh. Ver. Brün. XVI, p. 203. Cancuse. humeralis Fisch. Entom. Ross. II, p. 203 . . Russie. circumductus MÉNétr. Cat. Rais., p. 158. Europe orient. et mérid.

Delphinas ReICHE, An. Fr., 1869.

Lavergnei o REICHe, Tbid. mollis ReITt.

circumscriptus Cand. Monogr. III, p. 466. . Allem. mér. melanoderes Kres. Nat. Ins. IV.

circumductus ReDt. Fn. Austr.

austriacus Desbr. Abeille.

difformis LAc. Fn. Par. 640 .

France.

Lavergnei ㅇ ReICHe, Ann. Fr., 1869.

Bonvouloirei Reiche, Ann. Fr., 1869.

Pyrénées.

Ecoffeti Reiche; Cat. Grén., 1863, p. 80

France.

cavus Germ. Reis. Dalmat., 1817, p. 218. . . Dalmatie.

tenuis Brisout, Ann. Fr., 1866; p. 379 . . . Madrid.

procerus Illig. Mag. VI, p. 1.

Espagne.

escorialensis MuLs. Opusc. entom.

elongatus BRIsout, loc. cit.

tauricus CAND. Monogr. II, p. 470.

Crimée.

longicornis CAND. Elat. nouv. I, p. 50 .

Pyrénées.

pyræneus CAND. Ibid.

)

flavipennis CAND. Monogr. III, p. 473

Piémont. 
hispidus CAND. Ibid. . . . . . . . . Fr.mér.

Dejeani Casteln. Hist. nat. Ins. I, p. 244 . . ” cervinus CAsT. Ibid.

fuscicornis MoLs. Op. entom. VII.

agnatus REICHE, Ann. Fr. 1869 . . . . . . Alpes fr. fallax ơ Desbr. Abeille, 1870.

melanoderes Muls. Opusc. ent., 1855, VI, p.22.

)) fallax $†$ Dessr. Abeille VII.

epirus Stierl. Mitt. Schw. entom. Ges. IV, p. 474 .

frigidus Muls. Opusc. entom. VI, p. 21 . . . castanescens Muls. Ibid.

Albanie. Fr.mér. vestitus MuLs. Ibid.

var. Peragalloi ð REICHE, Ann. Fr., 1864, p. 247. cylindricollis MuLs. Ibid.

Bordeaux. oblongicollis Desbr. Mitt. Schw. ent. Ges. III. Alpes mar. uncicollis Perris, Ann. Fr., 1864, p. 285 . . . Escurial. hæmus Burss. Ibid., 1889, Bull. covt. . . . Turquie. mandibularis Dufour, Bull. Soc. Pau, 1843,

p. 41

† titanus MuLs. Opusc. entom. VII, p. 60.

Puzosi Dej. Cat.

canus Dufour, loç, cit.

pubescens MaNnerH. Humm. Essai VI, p. 6 gigas ReIтt. Entom. Nachricht, 1890, p. 249 . Monténégro. Revelierei Muls. Ann. Soc. lin. Lyon, 1874,

p. 416. virens Cand. Mém. Soc. Liége V, $2^{\circ}$ sér.

Corse. secessus CAND. Tbid.

Japon.

subcyaneus Motson. Bull. Mosc., 1866, I, p. 166.

Pyrénées. erosus (') Lewis, Ent. Monthl. Mag., 1879.

(1) Je ne connais pas les espèces suivantes données comme nouvelles, par M. Desbroschers des Loges. Leur valeur spécifique étant sujette à caution, je me borne 


\section{$2^{e}$ Groupe.}

Nouveau continent.

Brightwelli Kraby, Fn. bor. Am. IV, p. 146.

Amér. bor. arcticollis, oblongicollis MELSH. Pr. Ac. Phil. II. a canthus $\mathrm{S}_{A Y}$, Trans. Am. ph. Soc. VI, p. 178. flavangularis Horn, Trans. ent. Soc., 1871. maculicollis Lec. New. Sp., 1863.

opilinus CAND. Monogr. III, p. 435.

Ohio. cribratus LEC. Rep. of Chief of Engin. 1876 Colorado. axillaris Hork, Trans. Am. Ent. Soc. 1871, p. 316.

excavatus Мотsсн. Bull. Mosc., 1859, IV, p. 467. Californie. cucullatus SAY, Ann. Lyc. I, p. 264 . . . Etats-Unis. hypoleucus, longicollis, procericollis, ㅇ strigatus Metsh. Pr. Ac. Phil. II.

ferruginosus Eschs. Thon, Arch. II, 1, p. 33. Californie. fossularis Lec. Trans. Am. ph. Soc. X, p. 426. New Jersey. nigripilis Mots. Bull. Mosc., 1859, IV, p. 368. Califomie. scapularis $\mathrm{S}_{A Y}$, Trans. Am. ph. Soc. VI, p. 178. Etats-Unis. equestris LEC. Tbid. X, p.426 . . . . . . ” rufiventris Esch. Entom. I, 1822, p.71 . . Unalashlia. posticus Melsh. Proc. Ac. Phil. II, p. 158 . . Pensylvanie. debilitatus pour debilis (') Lec. Proc. Am.

Ph. Soc. 1848, p. 405 . . . . . . . . . Lac Hurney. rufifrons Randali, Bost. Journ. I, p. 6. . . Etats-Unis. reflexus Lec. Trans. Am. Soc. X.

limbatus Lec. Proc. Ac. Phil., 1868, p. 391 . . Californie. simplex LEO. Rep. of chief Engin. scissus LEC. Ibid.

à les citer en note. Ce sont : $\boldsymbol{A}$. depressifrons, Perrisi, villosulus, de Corse; interstitialis, nigerrımus, pallidipennis, midi de la lirance; distinctichorax, d'Espagne.

l'lusieurs rectifications de synonymie, principalement en ce qui concerneles espèces de Reiche, sont dues à M. du Buysson. Elles sont souvent motivées par les différences de sexes que l'on observe chez beaucoup d'especes de ce genre.

(') Nom déjà employé. 
vittiger Lkc. Trans. Am. phil. X, p. 427 . . . Orégon. orophilus HAR. Coleopt. Heft.V, p. 125 (Emend.). Montana. montanus Lec. Proc. Acad. Phil., 1866.

triundulatus Mann. Bull. Mosc. 1863, III, p. 222. Sithka. affinis CowPer, Canad. nat. II, 1865, p.61 . . Québec. mexicanus CAND. Monogr. III, p. 433 . . . . Mexique. campyloides CAND. Élat. nouv. II, p. 40. . . Chili.

\section{TRIBU XVI.}

\section{PYROPHORITES.}

Front plat ou concave, non rebordé en avant sur la ligne médiane; des taches vésiculeuses jaunes (lumineuses pendant la vie de l'insecte) vers les angles postérieurs du prothorax, chez presque tous.

Pyrophords.

ItuIG. Mag. d. Gesellsch. I, p. 141.

Hypsiophthalmus LATr. Stilpnus, Belania CAST.

Phanophorus SoL.

Pyrophorites à tarses ni dilatés ni lamellifères; mésosternum déclive; taille généralement grande ou moyenne; propres à l'Amérique.

\section{section 1.}

Vésicules phosphorescentes latérales ou angulaires; antennes dentées à partir seulement du $4 \mathrm{e}$ article; plus courtes que la tête et le prothorax; taille grande.

noctilucus Lns. Mus. Lud. Ulr., p. 82 . Amér. intertrop. divergens. Erichs. Thon, Arch.

nyctophanus GERM. Zeitschr. III.

phosphorescens Caster. Hist. nat. Ins. II.

plagiophthalmus Germ. Zeitschr. III, p. 14 . Jamaïque. strabus Germ. Ibid. . . . . . . . . . Mexique. luscus CAND. Élat. nouv. IV,p. 47 . . . . Guatémala. 
punctatissimus Blanchr. Voy. d'Orb. col. 138.

Bolivie.

$\delta$ elongatus. Bu. Ibid.

laticollis Bu. Ibid.

tuberculifer Eschs. Thon, Arch. I, II, p. 32 . Brésil.

hesperus Cand. Moñogr. IV, p. 18. . . . Cuba.

peliucens Escus. Thon, Arch. II, I. . . . Amér. intertrop. surinamensis StuRM, Cat., p. 47.

clarus Germ. Zeitschr. III, p. 18. . . . . . Andes.

angustus $\mathrm{BL}$. loc. cit.

cognatus SturM, Cat., p. 67.

indistinctus Germ. Zeitschr. III, p. 20 . . . Cayenne.

\section{Gection II.}

Antennes dentées en scie à partir du 40 article, généralement longues; prothorax ordinairement plus long que large, ses vésicules souvent diffuses sur les bords, parfois oblitérées.

syrius CAND. Élat. nouv. II, p.41 . . . . Costa-Rica. ignitus FABR. Mantiss. I, p. 172. . . . . . Colombie. fuscus Voet, Col. I, p. 116.

Salingeri Herbst, Käf. X.

bifossulatus CAND. Élat. nouv. I, p. 51 . . . Pérou. indicus Herbst, Füesl. Arch. V. . . . . . Vénézuéla. extinctus Iluig., Cand. Monogr. IV.

fulgidus Germ. Zeitschr. III, p. 24 . . . . . Brésil. I adumbratus Germ. Ibid.

melanurus CAND. Monogr. IV, p. 23 . . . Cayenne. occiduus Erichs. Col. Peruan, 1847 . .. . Pérou. mesochrous Germ. Zeitschr. IV, p.91 . . . Brésil. luminosus Irlig. Mag. I, 2, p. 149 . . . . . Antilles.

phosphoreus FABR. Syst. Eleuth.

var. pyralis GERM. Zeitschr. III.

lychnus CAND. Monogr. IV, p. 26 . . . . ”

acuminatus GERM., loc. cit. 
Jychniferus GERM. Zeitschr. III, p. 32 . . . Cuba. domingensis StuRM, Cat. var. phosphoreus BEAuv. Ins.

havaniensis CAST. Hist. Nat. Ins. I, p. 236 . ” causticus GerM., CAND., loc. cit. impressifrons $\mathrm{S}$ T. Cat.

pyrophanus ItLIG. Mag. 1, 2, p. 149 . . . . Brésil. angusticollis, acuminatus, quadricollis EscH. pyrrhoderus, lusciosus GeRM. Zeitschr. lucens ILLIG. Mag. acutipennis $\mathrm{CAST}_{\mathrm{AST}}$ Hist. Nat.

illuminans GerM. Zeitschr. III, p. 30. . . . ” fulvotomentosus Bu. Voy. d'Orb.

helvolus Germ., loc. cit. rufofuscus $\mathrm{S}_{\mathrm{T}}$. Cat.

stella CAND. Monogr. IV, p. 32 . . . Guatémála. physoderus Germ. Zeitschr. II, p. 36 . . Etats-Unis mér. ornamentum Gerr. Ibid. . . . . . . Mexique. caliginosus $\mathrm{Sт.} \mathrm{Cat.}$

o gemmiferus GeRM., loc. cit.

cœcus Germ. Zeitschr. III, p. 40 . . . . . Paraguay. hebes Germ. Tbid.

abnormis CAND. Monogr. IV, p. 36 . . . Nouv. Grenade. alychnus KrRscH, Berl. Ent. Zeitschr., 1873, p. 368. funale CAND. Monogr. IV, p. 37 .

Pérou. pyrotis GeRM. Zeitschr. III, p. 42 . . . . . ” lucificus GeRM. Zeitsch. MI, p. 43 . . . . . . ” maculicollis CAND. Monogr. IV, p. 38 . . . ” cincticollis GERM. Zeitsch. III, p. 44 . . . . ” sanguinicollis CAND. Élat. nouv. $\amalg, \mathrm{p} .41$. . Para. formosus CAND. Monogr. IV, p. 41. ... . . Brésil. melanoxanthus CAND. Élat. nouv. I, p. 51. . Vénézuéla. 


\section{Gection III.}

Caractères des premières sections, à antennes dentées à partir du $3 \ominus$ article, ce dernier semblable au 40 .

parallelus Gerr. Zeitschr. II, p. 45 . . . . Brésil mér. rubripes Bu. Voy. d'Orb.

crassus Blanch. Voy. d'Orb. Col., p. 141 . . Uruguay. luculentus GerM. Zeitschr. III, p. 46 . . . . Brésil.

facifer GERM. Tbid.

ignifer GERM. Ibid.

pyraustes GeRM. Ibid., p. 47.

\section{Eection IV.}

Vésicules postérioures; troisième article des antennes semblable au quatrième.

Gandezei Fauv. Bull. Soc. Norm. V, p. 11 . . Guyane. perspicillatus Germ. Zeitschr. II, p. 54 . Buenos-Ayres.

\section{gatection $\mathbf{V}$.}

Vésicules postérieures; antennes à article 3 plus court que le 4 e.

ocellatus GERM. Zeitschr. II, p. 49 . . . .

Chiti.

variolosus Sol. Gay, Hist. Chil.

conicicollis FatrM. Col. Chil.

leporinus CAVD. Monogr. IV, p. 47

Germari CAND. Ibid., p. 48

)

lampyris CAND. Ibid., p. 49

Tucuman.

limbatus CAND. Ibid.

Brésil.

tessellatus CAND. Ibid., p. 50.

»

spurius GeRM. Zeitschr. III, p. 56

Pérou.

nictitans ILlig. Mag. Nat. Fr., 1, 2, p. 146 .

Brésil.

fulgurans CAND. Élat. nouv. I, p. 51

Amazones.

scintillula CAND. Ibid. III, p. 90

Cayenne.

lucidus CAND. Monogr. IV, p. 52.

Para. 


\section{Section vi.}

Vésicules postérieures; $3 \theta$ article des antennes de même longueur mais plus étroit que le $4 \mathrm{e}$; prothorax toujours plus large que la tête, au sommet.

Ortizi CartD. Bull. Soc. Zool. Fr., 1889 . . . Grand-Chaco. candelarius GeRM. Zeitschr. II, p.59 . . . Brésil. $\delta$ diffusus Germ. Ibid.

ignitus Erichs. Th. Arch.

janus HerBst, Käf, X, p. 16 .

$\delta$ speculator IuLia. Mag. I.

i minor VOET, Col. I.

perspicax Guḱr. Voy. Coq., 1830, ПI, Col., p. 69.

Chili.

depressicollis BL. Voy. d'Orb., p. 143. . . Valle Grande. candens Gerr. Zeitschr. III, p. 65 . . . . . Brésil.

¿ planicollis Bu. loc. cit.

var. observator GeRM. loc. cit.

phosphoreus LinNe, Syst. Nat. 1, 2, p. 652.

cinerarius GerM. Zeitschr. III, p. 65 (pars).

cinerarius GERM. Zeitschr. III, p. 65 .

+ flammiger Germ. loc. cit., p. 52.

lucernula ILuig. Mag. Nat. Fr., 1, 2, p. 147.

amplicollis CAND. Monogr. IV, p.58 . . . . Guyane. nyctophilus GerM. Zeitschr. III, p. 51 . . Buenos-Ayres. commissator GerM. Ibid. . . . . . . . Brésil. nyctolampis GerMr. Ibid., p.54 . . . . . Uruguay. gibbicollis Blaiver. Voy. d'Orb.

lampadion Iturg. Mag. 1, 2, p. 144. . . . . Brésil.

$\delta$ scintillans Germ. loc. cit.

ignigenus Germar. Ibid. p. 57.

)

lineatus CAND. Monogr. IV, p. 62

Bolivie.

acutus CAND. Ibid.

Colombie.

pumilus CAND. Ibid., p. 63 .

lucidulus ILLTa. loc. cit. vitticollis GerM. Zeitschr. III, p. 60 . . . . Bahia. 
retrospiciens Lluia. Mag. I, p. 145. . . . .

Para.

ardens CAND. Monogr. IV, p.65. . . . . . Brésil.

niger Sou. Gay, Histor Chile V, p. 27 . . . . Chili.

dilatatus Sol. Tbid. . . . . . . . . ”

megalophysus PhiLrpi, Ann. Universit., 1881 ”

\section{gecion vir.}

Vésicules postérieures; corselet rétréci au sommet, les yeux très gros et le débordant, au moins chez le mâle.

raninus Eschs. Thon, Arch. II, p. 32 . . . . Brésil. exophthalmus GuEr. Voy. Coq.

+ brevicollis Eschs. Tbid.

† longipennis GerM., loc. cit. CAND. Mon.

cephalotus Bu. Voy. d'Orbigny.

buphthalmus Eschs. Thon, Arch. II, I, p. 32 . ”

microspilus Germ. Zeitschr. III, p. 71 . . . ”

boops Germ. Tbid. p. 69 . . . . . . . Jruguay. grossicollis BL. Voy. d'Orb.

\section{РноторноRUS.}

Cand. Monogr. IV, p. 72.

Mésosternum tuberculeux en arrière; tarses dilatés et cupulifères en dessous.

Jansoni CAND. Monogr. IV, p. 73 . . . . lles Viti. Bakewelli CAND. Ibid., p.74. . . . . Nouv. Hébrides.

\section{HIFO.}

CAND. Élat. nouv. III, p. 94.

Bords de la fossette mésosternale saillants, quatrième article

des tarses lamellé.

paciflcus CAND., loc. cit. . . . . . . Tonga-Tabou. 


\section{Paraphiledos.}

Cand. Élat. nouv. III, p. 92.

Sans vésicules lumineuses; corps large.

Thoreyi CAND., loc. cit., p. 93

Brésil.

\section{MEROPLINTHUS ('). \\ CAND. Mss.}

Forme et caractères des Pyrophorus; vésicules nulles ou peu distinctes; corps rouge, plus ou moins varié de noir.

trinotatus CAND. Élat. nouv. III, p. 91 . . . Brésil.

notatissimus CAND. Ibid. . . . . . . Surinam. ruber CAND. Ibid. II, p.41. . . . . . . Bahia. ophthalmicus Perty, Del. Anim., p. 21. . . Brésil.

Calais ophthalmica CAND. Mon. I, p. 243.

Monocrepidius abbreviatus GenM. Zeitschr. I.

Oophorus quadrilineatus. Des. Cat.

\section{IsCHIUS.}

CAND. Monogr. I, 1857, p. 195.

Sutures prosternales creusées; mésosternum saillant et horizontal dans sa portion postérieure; pas de vésicules lumineuses.

Gerstæckeri CAND. Monogr. I, p. 196 . . . Guyane. biplagiatus JANs. Cistula, 1882. . . . . . Équateur.

\section{HeLigMus.}

CaNd. Élat. nouv. I, 1865, p. 52.

Antennes lamellées; sutures du pronotum doubles et canaliculées au sommet; vésicules lumineuses pou distinctes.

glyphoderus CAND., loc. cit. . . . . . Brésil.

(1) Ne diffère en réalité des Pyrophorus que par un facies et une coloration difféerents. C'est un groupe qui mérite, toutefois, de porter un nom particulier. Le
Pyrophorus spurius n'y serait pas déplacé. 


\section{Pyrapractus.}

FatrM. Ann. Fr., 1884, p. 228.

Antennes très longues; pas de vésicules phosphorescentes.

sycophanta FArRM., loc. cit. . . . . . Madagascar.

\section{TRIBU XVII.}

\section{CORYMBITITES.}

Front dépourvu de carène transversale en avant, au moins sur la ligne médiane, plat ou concave, peu incliné; pas de vésicules phosphorescentes; hanches postérieures étroites; tarses simples, ni dilatés, ni lamellés.

\section{Corymbites.}

Latr. Ann. Soc. entom. Fr. III, p. 150.

Ludius Esons. Ctenicera pars Latr., Casteln. - Pristilophus pars, Prosternon pars, Diacanthus Latr., Germ. Selatosomus StePH. - Aphotistus Kurby. - etc.

Sutures prosternales rectilignes, fines ou légèrement dédoublées; mésosternum déclive.

\section{Gection $\mathrm{I}$.}

Antennes pectinées chez les mâles; leur second article seul petit. Corymbites p. d.

pectinicornis LINwi, Syst. Nat. II, p. 655 . . Europe. flabellicornis VoEт, Col. II, p. 120.

pyrenæus Charp. Hor. entom., 1825, p. 189. . Espagne. Kiesenwetteri BrIs. Ann. Fr., 1866.

Heyeri SAXES. Isis, 1838, p. 805.

† croaticus Genm. Zeitschr. IV, p.59 . - Europe centr. famelicus CAND. Élat. nouv. IV, p.47. . . . Darjeeling. 
cupreus FABr. Ent. Syst., p. 212

Europe.

castaneus Scop. Ent. carn., p. 93.

humeralis Duf. Exc. Val.. d'Oss.

v. ceruginosus FABR. loc. cit.

virens Schrank, Schr.Berl. Ges. Nat. II, p. 317.

)

ceneicollis Ourv. Journ. Hist. Nat. - CAND. Mon.

castaneus Scop, loc. cit.

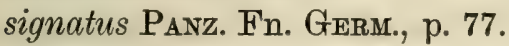

virescens Lin. ed. Gmel.

aulicus Panz., loc. cit.

cupreus var. Hensst, Käf. $\mathbf{X}$.

purpureus PodA, Ins. Mus. Græc. 1761 . . .

)

chrysomelinus MüLs. Zool. Dan. Prodr.

hoematodes FABR., CAND. Monogr. IV.

sanguineus Sulz. sulcatus Razumovski.

Scopolii Fuessl. Verz. - var. flavus GredL.

hæmapterus IuLiG. Mag. VI, p.13. . . . . Portugal.

castaneus LrNn. Syst. Nat. II . . . . . . Europe.

flavopectinicornis DE GEER, Mém.

sulfuripennis Germ. Zeitschr. IV, p. 55. . . Italie, Esp. affinis CASTELN. Hist. Nat. I, p. 239.

v. Zenii Rosenh. Thier. Andal. p. 138.

Eschscholtzi FaLderm. N. Mém. Mosc. IV, p. 173 . . . . . . . . . . . . . Perse.

melas KöNIG, Hor. Entom. Ross. 1887, p. 352 . Caucase.

$\mathbf{t} \in \mathbf{S t a c e i p e n n i s ~ M o т s c r . ~ S c h r e n c k ~ R e i s . ~ I I , ~}$

p. 109. . . . . . . . . . . . Songarie.

var. pacificus Mots. Tbid.

Bœberi Germ. Ins. Sp. nov., p. 51 . . . .

Sibérie.

quadripustulatus Fisch. Ent. Ross. II, p. 202.

Pippingskoldi ManN. Bull. Mosc. IV, p. 285.

Ajan.

Kendalli Krrb. (') Faun. bor. Amér. IV, p. 149. Lac Supérieur. anchorago RaNd. Bost. Journ. ПI, p. 5.

(') Considéré par quelques-uns comme var, du virens. 
vernalis Hentz, Joum. Acad. Phil. V, p. 374.

Canada.

Bection II.

Antennes pectinées chez les mâles; le 3e article aussi petit que le second. Orithales KIES.

serraticornis $\mathrm{P}_{\triangle \mathrm{XK}}$. Fn. suec. III, p. 21.

Suède.

\section{Gection 111.}

Antennes simples dans les deux sexes; $3 \theta$ article semblable au suivant; corps généralement étroit et allongé. Corymbites GERMr. Actenicerus, Liotrichus pars KIESENW.

resplendens Eschs. Thon, Arch. $\Pi, 1$, p. 34. . Canada. cerarius RAND. Bost. Journ. II, p. 7.

v. viridis GerM. Zeitschr. IV, p. 61.

Racinei Cн. Rev. Zool., 1852, p. 578.

sjælandicus MüLc. Fn. Fridr., 1764 . . Europe, Am.bor. mubilus Schranck, Bayersch. Reis., 1786.

tessellatus FaBr. Ent. Syst., Kies., Cand. Monogr.

viridis SAY, Ann. Lyc. I, p. 255.

cuprascens Lec. Trans. Am. Soc. X.

var. assimilis GYLI. Ins. suec.

Paulinoi Desbr. Ann. Fr. Bull., 1873, cxvin . Portugal. orientalis CAND. Élat. nouv. TV, p. 48. . . . Japon. selectus CAND. Ibid. I, p. 53 . . . . . . . ” pruinosus Mотs. Etudes entom., 1860 . . . » atropurpureus Melsh. Proc. Acad. Phil. II, p. 215. . . . . . . . . Pensylvanie. fusculus LEC. List. Col. N. Amer., 1863 . . . Etats-Unis. angustulus Moтsch. Bull. Mosc., 1859. jaculus Lec. Trans. Am. ph. Soc. X, p. 447 . Californie. cribrosus Lec. Tbid.

subcanaliculatus Motsch. Bull. Mosc., 1859.

obscurus LEC., loc. cit., p.442 . . . . . protractus Lec. Proc. Acad. Phil., 1859, p. 85 . Orégon. 
pyrrhos HeRBst, Käf. X, p. 30 . . . . . . Etats-Unis.

vagans Mresh. Proc. Acad. Phil. II, p. 153.

+ cequalis MeLsH. Ibid.

+ pyrrhicus HaLder. Ibid. I, p. 313.

bivittatus MELSH., loc. cit. II, p. 219

)

longicornis HorN, Trans. Am. phil. Soc., 1870,

p. 79

cylindriformis Herbst, Käf. X, p. 93 . . . États-Unis.

o appressifrons $\mathrm{SAY}, \mathrm{Ann}$. Lyc. I, p. 267.

+ brevicornis $\mathrm{SAY}_{\mathrm{A}}$, Ibid.

+ parallelipipedus Germ. Zeitschr. IV, p. 66.

+ submetallicus GerM. Tbid., p. 72.

Gopei Horn, Trans. Ent.Am. Soc., 1861, p. 319. Virginie. teres LEc. Proc. Ac. Philad., 1866, p. 392. . . Californie. furtivus Lec. Trans. Am. phil. Soc. X, p. 442 . Orégon. limoniiformis Honn, Trans. Am. ent. Soc., 1871, p. 320.

Dacota. divaricatus LEc. Trans. Am. phil. Soc. X, p. 446.

$\ddagger$ crassus LEC. Tbid.

floridanus CAND. Elat. nouv. IV, p. 51 . . . Floride. fulvipes Bland, Ent. Soc. Phil., 1864, p. 354 . Virginie. signaticollis MeLsh. Proc. Ac. Phil. II, p. 216. Etats-Unis. appressus RAND. Bost. Journ. II, p. 11 .

) mirificus Lec. Agass. Lake Super., p. 228.

trapezium Lec. Proc. Acad. Phil., 1866,p. 392. volitans Eschs. Thon Arch. II, I, p. 34

Texas. vulneratus LEC. New. Sp. Col., 1863, p. 86. Gebleri Geblex, Bull. Mosc., 1847, p. 412 (Athous).

Altaï.

umbricola Eschs. Thon Arch. II, I, p. 34 . I. Aleutiennes. caricinus GeRM. Zeitsch. IV, p. 63.

lobatus Mav. Bull. Mosc., 1843, II, p. 243.

telum Lec. Trans. Am. ph. Soc. X, p. 445.

rudis Mотsch. Bull. Mosc., 1859. 
truculentus CAND. Élat. nouv. IV, p. 50. . . Californie. xanthomus Hors, Trans. Am. ent. Soc., 1871, p. 19 .

mœrens Lec. Proc. Acad. Phil., 1866, p. 392 gracilis $\mathrm{C}_{A N D}$. Élat. nouv. IV, p. 51 ) quercus Ourv. Entom. II, 31, p. 51 . . . . . Orégon. Californie. Europe. Gyllenhali Reiche, Ann. Fr., 1860. Bull., p. 109. incanus Grul. Ins. suec. III, p. 14. pallipes $\mathrm{P}_{\mathrm{AY}}$. Fn. suec. III, p. 14. rufipes Sтерн. Mlustr. Brit. III, p. 272.

var. ochropterus, testaceus STEPH., loc. cit.

Ghristophi Kres. Deutsch. Entom. Zeitschr., 1879, p. 145 . . . . . . . . .

Amur. atricornis CAND.

coarctatus CAND. Ann. Mus. civ. Gênes XII, p. 137.

Java. affinis PAYK. Fn. suec. III, p.12. . . . Europe, Sibérie. erythropus Fisch. Ent. Ross. II, p. 203.

sibiricus Germ. Ins. Sp. Nov., p. 58.

nivicola Kres. Berl. Zeitschr. 1865, p. 391 . . Expagne.

tibiellus CHEvR. Rev. Zool., 1865, p. 352.

angustulus Kres. Nat. Ins. IV, 2, p. 291

Europe.

Putonis Dessr. Ann. Fr., 1873, Bull. cxvIII tarsalis Melsh. Proc. Acad. Phil. II, p. 157. spinosus Lec. Tr. Am. Soc. X, p. 447 . . . insidiosus Lec. Ibid. . . . . . . . . Maryland. Canada. angularis LEc. Ibid., p. 449 . . . . . . . Orégon. mendax Lec. Tbid. . . . . . . . . Canada. falsificus LEC. Tbid. . . . . . . . . États-Unis. umbripennis LEC. Report. Surv. Pacif., 1857,

p. 46

Orégon. gracilior LEC. List. Am. Col., 1863, p. 49. mubilipennis LEC. Trans. Am. Soc. X, p. 441. fuegensis FatrM. Ann. Fr., 1885, p. 46 . . Terre-de-Feu. 
Bection IV (1).

Antennes plus ou moins dentées, leur troisième article plus court et plus étroit que le quatrième.

strangulatus Wнгте, Voy.Ereb. a. Terror, p. 7. N.Zélande. antipodum CAND. Monogr. IV, p. 133. . . . ” agriotoides Sharp, Ann. a. Magaz. May 1877. dubius Share, Ibid. . . . . . . . . . ” irregularis Sharp, Trans. Acad. Dublin 1886, p. 401. . . . . . . . . . . . . . ” mundus Sharp, Ibid. . . . . . . . . . ” corruscus Karsch, $\left({ }^{2}\right)$ Berl. entom. Zeit., 1881, p. 5 . . . . . . . . . . . . . . . Hawä̈. sulcicollis $\mathrm{S}_{\mathrm{AY}}$, Trans. Am. phil.Soc. IV, p.168. Etats-Unis. rupestris GerM. Zeitschr. IV, p. 66 . . . . Orégon. morulus Lec. New. Sp. Col. I, p. 85 . . . . Canada. brunnipes Bland, Proc. ent. Soc. Phil., 1864, p. 67. montivagus RosenH. Beitr. I, p. 15 . . . . Tyrol. acutus (Athous) Muls. Opusc. VI. sagitticollis Eschs. Thon Arch. II, I, p. 34. . Alaska. angusticollis MaNn. Bull. Mosc., 1843, II, p.241. Sithka. ochreipennis Lec. New. Sp. Col., 1863, p. 85 . Californie. trivittatus Lec. Trans. Am. phil. Soc. X, p. 443 . États-Unis. colossus Lec. Proc. Acad. Phil., 1561, p. 348 . Californie. maurus Lec. Trans. Am. phil. Soc. X, p. 444 . Orégon. cribrosus Lec. Tbid., p. 443 . . . . . . . . Californie. anthrax Lec. Proceed. Ac. Phil., 1861, p. 349. æthiops Herвst, Käf. X, p. 70 . . . . . . Etats-Unis. nigrans CAST. Hist. Nat. Ins. I.

loevigatus HenBst, loc. cit.

(1) Correspond à la section $\mathrm{V}$ de la monographie, la précédente ayant été distraite du genre sous le nom rétabli de Pristilophus.

$\left({ }^{2}\right)$ Je ne connais pas ces quatre dernières espèces et je ne sais si elles sont à leur place dans cette section. 
insitivus Germ. Ins. Sp. nov., p. 44 . . . . Hongrie. depressus GERM. Fn. ins. 7, 9.

Gougeleti Farrur. Ann. Fr., 1859, bull., p. 151.

dauricus Motschs. Schr. Reis. II, 1860, p. 110.

Sibérie. ferrugineipennis CAND. Deutsch. Ent. Zeits., 1879, p. 281. . . . . . . . . . Amur. ligneus CAND. Ibid. . . . . . . . . . . ” patruus Hexd. Tbid., 1883. . . . . . . Vladivostok. notabilis CAND. Mém. Sc. Liége $\mathrm{V}, 2^{\circ}$ sér. . J Japon. serrifer CAND. Ibid. puncticollis Мотsch. Bull. de Moscou, 1866, I, p. 167. speculifer CAND. Élat. nouv. IV, p. 48 ) melancholicus FABR. Ent. Syst. suppl., p. 139. Europe sept., Sibérie.

var. scabricollis Eschs. Entomogr. 1822, p. 68. nigrita Mlavn. Bull. Mosc. 1852, IV, p. 289 . . uncinatus Escrs. Thon Arch. I, II, p. 34. Daourie. lævicollis MLANn. Bull. Mosc. 1852, p. 288 Kamschatha. + gracilis Motscr. Schr. Reis.

anxius GeBL., Bull. Acad. Pétersh. 1843, I, p. 38. coerulescens Мотsсн. Schr. Reis., 1860, p. 109. spretus Marn. Bull. Mosc., 1852, 4, p. 285 . . punctatissimus M[ÉNÉTr. Midd. Reis., 1851 p.53. Lederi Heyd. Verh. Ver. Brünn XVI, p. 204.

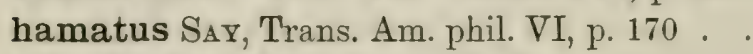
propola Lec. Trans. Am. phil. X, p. 437. furcifer LEC. Tbid. nubilus LEC. Ibid.

nigricollis Bland, Proc. ent. Soc. 1864, p. 68. triundulatus RAND. Bost. Journ. II, p. 12 . . Sibérie. nebraskensis BLAND, loc. cit.

medianus Germ. Zeitschr. IV, p. 71 rubidipennis LEc. Trans. Am. Soc. X. sericeus GeBL. Ledeb. Reise II, p. 83.

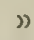

))

)

Cancase. Etats-Unis. ) )

Sibérie. 
tessellatus Linv. Syst. Nat., p. 406 . . . . Europe. holosericeus OL. Ent. CAND. Monogr. IV.

rufipes var. De GeER. Mém. Ins.

undulatus Herbst, Käf. X.

v. bifasciatus Küster, Käf. Europ.

nubilipennis Germ. Zeitschr. IV, p. 69 . . . Russie mér. chrysocomus Germ. Tbid. . . . . . . . Hongrie.

fallax SAy, Trans. Am. ph. Soc. VI, p. 170 . . Canada. bombycinus GERM. Zeitschr. IV, p. 70 . . . Californie. semiluteus Lec. Trans. Am. Soc. X, p. 445.

elegans CAND. Élat. nouv. III, p. 97 . . . . Californie. alpestris MÉnÉTr. Cat. rais., 1832, p. 155 . . Caucase. profugus FaLd. Faun. Transc. I, p. 176.

globicollis Germ. Ins. Sp. nov. CaNd. Monogr. IV.

\section{aection $\mathbf{v}$.}

Antennes dentées à partir du $4^{\mathrm{e}}$ article, le $3{ }^{\ominus}$ obconique plus grêle et ordinairement plus long que le suivant; prothorax généralement large; élytres élargies en arrière et souvent métalliques. Diacanthus GERM.

leucaspis Germ. Zeitschr. IV, p.73 . . . Orégon. æneus Linn. Syst. Nat. $10^{\mathrm{m}}$ ed., p. 406 . . . Europe. impressus MaRSH. Ent. brit. I, p. 387. var. caucasicus MÉnétr. - germanus LIN. coruleus Herbst, - cyaneus Marsh. nitens Scopp. - viridinitens VoEт. Col.

æneomicans Farrm. Ann. Fr., 1889, p. 33 . Chine centrale. puberulus CAND. Deuts. Ent. Zeitschr. 1879,

p. 282

Amur. depressus CAND. Tbid. . . . . . . . . . Sibérie. plorator CAND. Élat. nouv. IV, p. 48 . . . . Turkestan. amplicollis Germ. Zeitschr. IV, 1843, p. 80. . Pyrénées. pyroneus Des. Cat.

atratus BAILL. Bull. de Moscou, 1878, p. $293 . \quad$ Sibérie, Afganistan. 
violaceus CAND. Élat. nouv. IV, p. 49 Arménie. songoricus KraAtz, Deutsch. Ent. Zeitschr., 1879, p. 283. . . . . . . . Sibérie. informis KraAtz, Ibid. . . . . . . . ” rugosus GerM. Fn. Ins. Europ. 18, p. 7 . . . Alpes. v. confluens GEBL. Ledeb. Reis. II, p. 3. æripennis Kтrв. Faun. bor. Amer. IV, p. 150 . Etats-Unis. apropinquans RAND. Bost. Journ. II, p. 5.

tinctus Lec. Proc. Ac. Phil., 1859, p. 85.

splendens Ziegl. Proc. Ac. Phil. II, p. 44 . . Pensyluanie. nigricornis $P_{A N Z}$ Fn. Germ., 1799, 61, p. 5. . Europe, Sibérie, Amérique boréale.

metallicus PAYK. Fn. suec. CAND. Monogr. nitidulus LEC. Trans. Am. Soc. X.

impressus FABR. Ent. syst. 1, 2, p. 223

) Przewalskyi Könia, Horæ Soc. Ent. Ross.,

1889, p. 535. . . . . . . . Amdo, Asie centrale. obscuroæneus KöNı, Ibid. . . . . . . » Ssemenovi Könta, Ibid. . . . . . . . . ” Roborowski KöNı, Ibid. . . . . . . . » aratus Lec. Trans. Am. Soc. X, p.439. . . . États-Unis. latus FABR. Syst. Eleuth. II, p. 232. . . . . Europe. v. gravidus, melo, GerM., germanus Or. pasticus, saginatus MÉNÉTR. Cat. rais.

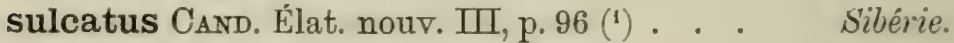
centralis CAND. Élat. nouv. III, p. 96 . . . Moupin. inflatus $S_{A Y}, A_{n}$. Lyc. I, p. 258. . . . . Etats-Unis. v. glaucus GERM. Zeits. IV. simillimus Motsch. Bull. Mosc., 1859. corpulentus CAND. Deutsch. Ent. Zeitschr., 1879, p. 283.

Sibérie. Baeri Kusch. Horæ entom. Ross., 1861, p. 55 . Sibérie or. 
puerilis Cand. Mém. Soc. Sc. Liége $V, 2^{\circ}$ sér. . Japon. mondulus Lewis, Ent. Montl. Mag., 1879 . . 》 paradoxus KöNıt, Hor. Soc. Ent. Ross., 1887, p. 353 . carbo Lec. Trans. Am. Soc. X, p. 439. Sergiopol. Orégon. lateralis LEC. Tbid.

Breweri Hons, Trans. Ent. Soc., 1871, p. 321 - C Californie. conjungens Lec. Trans. Am. Soc. X, p. 440. proeses (Drasterius) CAND. Élat. nouv. I.

Lecontei CAND. Élat. nouv. III, p. 94 bipustulatus LrNNE, Syst. Nat. I, 2, p. 652 . bimaculatus Fourcr. Ent. Par. punctatus VozT, Col. II.

humeralis Мотsсн. Schr. Reis., 1860, ПI, p. 110. cinctus $\mathrm{P}_{\triangle Y Y K}$ Faun. Suec. III, p. 10 inunctus Lac. Fn. Par. I, p. 642.' rotundicollis $S_{A Y}, A n n$. Lyc. I, p. 259 . . . Vermont. diversicolor Eschs. Thon. Arch. II. russicollis $\nabla$. , sticticus Germ. Zeitschr. IV. urostygma CAND. Élat. nouv. IV, p. 50 Californie. cruciatus Linvé, Syst. Nat. Ed. 10, p. 404 . . Europe, Europe. Altaï. Europe, Amérique boréale. pulcher Lec. Trans. X. จ. festivus Leo. Report Expl., 1857.

Whitei CAND. Monogr. IV, p. 171 Himalaya. Suckleyi Lec. Report. Surv. Pacif., 1857, p. 46. semivittatus SAY, Journ. Acad. Phil. III, p.174. tristis CAND. Monogr. IV, p. 172. hieroglyphicus SAY, Trans. Am. ph. Soc. VI, p. 172.

Canada. xanthopterus CAND. Monogr. IV, p. 175. Melbourne. compsorhabdus CAND. Ibid. . . . . Moreton Bay. litura CAND. Élat. nouv. III, p.95 . . . . . Victoria. ambiguus CAND. Tbid., p. 96 . . . . . . »

Orégon. ) 
rufipennis MaC Leay, Tr. N. S. W. II, p. 258 . Queensland. nigrinus Mad LeaY, Tbid.

Gection WI.

Troisième article des antennes plus court que le suivant; hanches mésothornciques subcontiguës; tarses allongés, grêles; prothorax court; élytres dilatées en arrière; s. g. Paranomus Kies.

guttatus Germ. Fn. Ins. Eur. 21, p. 5. . . . Alpes. pictus CAND. Monogr. IV, p. 177. . . . . Canada. costalis PAYK. Fn. suec. II, p. 37 . . . . . Laponie, Sibérie, Canada. parvicornis Mann. Bull. Mosc., 1853, trim. III. vagus LEC. Trans. Am. Soc. X.

estriatus LEc. loc. cit., p. 434 . . . . . . Canada. decoratus ManN. Bull. Mosc., 1853, III, p. 229. 1. Kadiack. singularis MaNN. Ibid., 1852, trim. IV, p. 290 . Ajan.

NoTHODES.

LEC. Class. Col. N. Amér. I, 1861, p. 171.

Corymbites à facies de Limonius, à lobe du prosternum plus court, ses sutures canaliculées au sommet.

dubitans LEC. (Limonius) Trans. Am. phil. Soc. X, p.433 . . . . . . . . . Etats-Unis.

Pristillophus.

(Latr.) Germ. Zeitschr. IV, p. 82.

CaNd. Bull. Soc. ent. Belg., 1890.

Corymbites Sect IV in CaNd. Monogr. IV.

Troisième article des antennes plus court que le 40. Prothorax généralement allongé, ses bords dédoublés par la carène des angles postérieurs prolongée.

viduus Bонем. Ins. Caffr. I, II, p. 410 . . . . Cafrerie. Beliophorus viduus CAND. Monogr. I, p. 364. 
summus CAND. Monogr. IV, p. 123 .

Cafrerie.

mucronatus CAND. Ibid.

)

)

aurulentus CAND. Ibid.

Cap.

sericans GERM. Zeitschr. IV, p. 87

Cafrerie.

attenuatus Boнем. Ins. Caffr. I, II, p. 412

》)

leptus CAND. Monogr. IV, p. 126.

)

macilentus CAND. Ibid.

Cap.

servus GeRM. Zeitschr. IV, p. 88

》

famulus GerMr. Ibid.

)

amaurus CAND. Monogr. IV, p. 129 . . . . ”

Peringueyi CAND. Élat. nouv. IV, p. 49 . . . ”

longus CAND. Tbid., p. 50 . . . . . . . . ”

piciventris CAND. Ibid. . . . . . . . . ”

rhomalocerus CAND. Monogr. IV, p. 130 . . Natal.

pseudalaus CAND. Tbid. . . . . . . . . Cafrerie.

velutinipes CAND. Ibid., p.131 . . , . . . Natal.

semicribrosus FaIRM. Ann. Fr., 1887, p. 153 . Zanzibar. luzonicus CAND. Élat. nouv. I, p. 53 . . . 1. Philippines. bengalensis CAND. Ibid. IV, p. 48 . . . . Bengale. morosus CAND. Ibid. III, p. 95 . . . . . Cochinchine. cirratipilis CAND. Ibid. I, p. 54 . . . . . . Malacca.

Nota. - Le P. impurus Germ. (Lins, ent. III, p. 180) n'appartient probablement pas au genre tel qu'il est entendu ici.

\section{Melanactes.}

Le Conte. Trans. Am. ph. Soc. X, p. 493.

Pristilophus Germ. Zeitschr. IV.

Sutures prosternales légèrement courbes; bords de la fossette mésosternale horizontaux. Américains.

procerus LEC. loc. cit. États-Unis. piceus De GEER, Ins. 4, p. 162

lovigatus FABr. Ent. Syst. Germ. Zeitschr.

morio var. SAY, Trans. Am. Soc. VI.

femoralis Melsh. Proc. Ac. n. sc., p. 2. 
densus Lec. Trans. Am. phil. Soc. X, p. 494. . Californie. Schaumi CAND. Elat. nouv. I.

morio FABR. Entom. Syst. suppl., p. 138. . . Etats-Unis.

lacunosus FABr. Syst. Eleuth. II, p. 224.

puncticollis Lec. Proc. Acad. Nat.Sc. VI, p.68. Missouri. consors Lec. Trans. Am. ph. Soc. X, p. 495. . Nebraska. Reichei Germar, Zeitschr. IV, p. 85 . . . . Georgie.

Chrosis.

CAND. Monogr. IV, p. 185.

Pristilophus ERICHS.

Bords de la fossette mésosternale horizontaux; tarses villeux en dessous; habitat spécial.

trisulcata Erichs. Wiegm. Arch., 1842 . . . Tasmanie. exarata CAND. Monogr. IV, p. 186. . . . N. Hollande. illita CAND. Ibid. . . . . . . . . . . . » barbata CAND. Élat. nouv. I, p. 54 . . . . ” Lansbergei CAND. Ibid. III, p. 97 . . . . ” ” æneola CAND. Tbid. I, p. 54. . . . . . N. Zélande. E. olivascens WHITE.

elongata Sharp, Ann. and Mag. may, 1877. . ” polita Sharp, Ibid. . . . . . . . . . ” reversa Sharp, Ibid. . . . . . . . . ” livens (Sharp) Broun, New Zeal. J. Sc., 1882,

p. 301. . . . . . . . . . . . . . »

violacea, SHARP.

valida Sharp, Ent. Montley Mag., 1881, p. $661 . \quad$ ” setigera SHARP, Ibid., p. 682 . . . . . . 》 brevicollis SHARP, Ibid. . . . . . . . » castanea Sharp, Tbid., p. 683. . . . . . . ” certa SHARP, Ibid. . . . . . . . . » fulvipes SHaRP, Ibid. . . . . . . . . . ”

(Note.) Je n'ai point vu ces dix espèces décrites par M. Sharp. 


\section{ApHileus.}

Cand. Monogr. I, p. 184.

\section{Dorcostoma NewM.}

Grand, large, déprimé, revêtu de poils raides; mandibules très saillantes; facies de Lucanide.

lucanoides CAND. loc. cit. . . . . . Australie or.

Jansoni Newr. Trans. Ent. Soc. n. sér. IV, p. 52.

var. depressus CAND. loc. cit.

Chrostus.

CAND. Élat. nouv. II, p. 42.

Sutures prosternales canaliculées au sommet.

quadrifoveolatus CAND. loc. cit. . . . . . Paroo River.

Anrychus.

Pascoe, Ann. and Mag., 1876.

Mésosternum à fossette large; opaque, facies de Lacon.

Gandezei Pascoe, Tbid. . . . . . . N. Zélande, Iles Pitt et Chatam.

\section{Hapatesus.}

Cand. Monogr. IV, 1863, p. 188.

Sutures prosternales canaliculées et concaves.

hirtus CAND. Tbid. . . . . . . . . . . N. Hollande.

hirtellus CAND. Élat. nouv. III, p. 98 . . . . N. Guinée. pretiosus CAND. Notes Leyd. Mus., 1887, IX,

p. 285. . . . . . . . . B. Carpentarie.

\section{AsYMPHUS.}

Sharp, Tr. Soc. roy. Dublin, 1885, p. 401.

mundus Sharp, loc. cit. . . . . . N. Zélande. 


\section{OXYLASMA.}

Broun, New Zeal. J, Sc., 1881.

pannosum Broun, loc. cit., p. 679 . . . . . N. Zélande. tectum Broun, Ibid., p. 680 . . . . . . . " "

basale Broun, Man. n. Z. Col.1886. . . . . ”

PSOROCHROA.

Brodn, Man. New Zeal. Col., 1885.

granulata Broun, loc. cit. . . . . . N. Zélande.

PARINUS.

Sharp. Ann. a. Mag. Mai, 1877.

villosus SHARP, loc. cit.

ITODACNUS.

Share, Ir. Trans. roy. Soc. Dublin, 1885.

gracilis ShaRP, loc. cit. . . . . . . Hawnï. corruscus KarSCH, Berl. Ent. Zeits., 1881 . . ”

\section{TRIBU XVIII.}

\section{CRÉPIDOMÉNITES.}

Front et hanches postérieures des Corymbitites, mais les articles 2-4 des tarses élargis et plus ou moins cordiformes.

ANATssus.

CAND. Monogr. I, p. 187.

Articles 1-4 des tarses dilatés et lamellés.

tarsalis CAND. loc. cit., p. 188 . . . . . N. Grenade. 


\section{Melantho.}

Casteln. in Silberm. Rev. entom. IV, p. 10.

Articles 1-4 des tarses très dilatés, munis de brosses en dessous.

Klugi CAST. loc. cit. . . . . . . . . . Madagascar.

v. Candezei F ArRM. Le Natural., 1880, p. 175.

v. costicollis CAST., loc. cit.

v. Raffrayi FAIRMr. Ann. Fr., 1884, p. 227.

Crepidonenus.

Erichs. Wiegm. Arch., 1842, 1, p. 140.

Ludius Borsd. Fn. Océan., p. 108.

Caractères des Corymbites; les tarses à 1er art. allongé dilaté en avant, tomenteux en dessous, ${ }^{\circ}$ plus court, également tomenteux et muni d'une sorte de ventouse en dessous, $3 \ominus$ et 4 e subcordiformes et plus ou moins lamellés.

fulgidus Erichs. Wiegm. Arch., 1842, 1, p. 140. Tasmanie. Lansbergei CAND. Élat. nouv. IV, p. 51. . . Sidney. sulcatus CAND. Ibid. II, p.43. . . . . . . Australie. subopacus CAND. Ibid., p. 44. . . . . . . »

decoratus Erichs. Wiegm. Arch., 1842, 1, p. $141 . \quad$ ” tæeniatus Erichs. Ibid. . . . . . . . » Georgei CAND. Élat. nouv. II, p. 43. . . . . ” Pulsi CAND. Ibid. . . . . . . . . . . » luteipes Boнem, Eugen. Resa, 1858, p. 70 . . Sidney. æneus CAND. Élat. nouv. II, p. 43 . . . . . Australie. australis Borsd. Voy. Astrol. Col., p. 108 . . ” metallescens CAND. Monogr. IV, p. 197. . . ” Victoriæ CAND. Ibid. ... . . . . . . ” seniculus CAND. Monogr. IV, p.198 . . . . Sidney. testaceus CAND. Ibid. . . . . . . . . . Australie. Adelaidæ CAND. Ibid., p. 197. . . . . . . » filiformis CAND. Tbid. . . . . . . . . . ” specularis CAND. Élat. nouv. IV, p.52 . . Tasmanie. 
rotundicollis CAND. Ibid. II, p. 44. . . . Australie. ovalis CAxd. Notes of Leyd. Mus. IX, p. 288. Carpenturia. hirtus CAND. Monogr. IV, p. 195 . . . . . Adélaïde. cordifer CAND. Élat. nouv. II, p. 43. . . . . Victoria. minimus CAND. Ibid., p. 44 . . . ... Adélaïde.

\section{Metablax.}

Cand. Coleopt. Heft. V, 1869.

Blax CAND. Monogr.

Mésosternum horizontal; $4^{\circ}$ article des tarses non dilaté; écusson arrondi.

acutipennis Whтte, Voy. Ereb. and Terror.

Zool., p. 7 . . . . . . . N. Zélande. cinctiger $W$ HITE, Ibid.

approximans WHтte, Ibid. . . . . . . . . » Brouni Sharp, Ann. a. Magaz. May 1877. . . ”

\section{OpHIDIUS.}

\section{Cand. Monogr. IV, p. 203.}

Fossette mésosternale ouverte en avant, ses bords perpendiculaires. elegans CAND. Monogr. IV, p. 204 . . . . . Victoria. histrio Borsd. Fn. Océan., p. 105 . . . . . Australie. dracunculus CAND. Monogr. IV, p. 204. . . ” serricornis CAND. Élat. nouv. II, p. 44 . . . Sidney.

\section{TRIBU XIX.}

\section{ASAPHITES.}

Hanches postérieures étroites; articles 2 et 3 des tarses lamellés ou sublamellés, le $4^{\theta}$ petit. 
AsapHes.

KrRBy, Fn. bor. Am., p. 146.

Hemicrepidius, Diacanthus Germ. Athous Metshem.

Agrypnus CHEvR.

hirtus CAND. Élat. nouv. I, 1865, p. 54 . . . Californie.

carbonatus Lec. Proc. Acad., 1860, p. 320 . . Orégon.

coracinus CAND. Monogr. IV, p. 214.

morio Lec. Trans. Am. phil. Soc. X, p. 450 . . Californie.

o verna CAND. Monogr. IV, p. 212.

dilaticollis Мотsсн. Bull. Mosc., 1859, IV, p. 368. . . . . . . . . . . . . . ”

tumescens Lec. Proc. Acad., 1861, p. 348 . . ”

oregonus Lec. lbid. . . . . . . . . . Orégon.

soccifer Lec. U. S. Surveys, 1876, p. 516. . . N. Mexique.

indistinctus Lec. Trans. Am. ph. Soc. X, p. 451. Georgie. decoloratus SAY, Trans. Am.ph. Soc.VI, p. 180. Etats-Unis or.

hemipodus + SAY, Ann. Lyc. 1, p. 254.

corporosus ${ }_{+}$Germar (Diacanthus) Zeitschr. IV.

cereus + Melsh. (Athous) Proc. Acad. 11, p. 156.

ceneolus of MeLsh. Ibid.

Memnonius Herbst, Käf. X, p. 29. . . . . ”

baridius SAY, Trans. Am. Soc. VI, p. 176.

Thomasi Germ. Zeitschr. I, p. 213.

ruficornis KTRBY, Fn. bor. Am., p. 146.

brevicollis CAND. Monogr. IV, p. 212.

bilobatus Say, Trans. Am. Soc. VI, p. 164.

melanophthalmus ${ }^{\circ}$ Mecs. (Athous) Proc. Acad. 11.

cavifrons $\delta$ MELSH. Ibid.

tener Led. Trans. Am. ph. Soc. X.

consentaneus ${ }_{+}$LEc. Mbid.

planatus ㅇ. LEC. Ibid.

Lecontei CAND. Élat. nouv. IV, p. 52 . . . . Californie.

pictipes CHevt. Magas. d. Zool., 1843, p. 230 . Mexique. 
instabilis CAND. Monogr. IV, p. 218 . . . . Mexique.

longicollis CAND. Tbid. . . . . . . . ”

flavipes CAND. Ibid., p. 219 . . . . . . . . "

deceptor CAND. Ibid. . . . . . . . . ”

leucostygma CAND. Mbid., p. 220 . . . . . "

longipennis CAND. Ibid. . . . . . . . . N. Grenade.

amœnus Phwipr, Ann. Univ. Chile V, 1861,

p. 743. . . . . . . . . . . Chili.

\section{Parasaphes.}

CAND. Élat. nouv. III, p. 101.

Front plat, bifovéolé; prothorax fortement sillonné; sutures du prosternum larges, brillantes, non creusées au sommet.

elegans CAND. loc. cit. . . . . . . . Queensland.

\section{Compsoctenus.}

Phirippi, Ann. Univ. Chili V, p. 743.

Front biimpressionné; prothorax sans sillon; sutures prosternales larges, brillantes.

Asaphes CAND. Élat. nouv. II, p. 44.

elegans PHILIPPI, loc. cit.

Chili.

\section{TIBIONEMA.}

Sourer in GaY, Histor. de Chile, V, Zool., p. 30.

Alaus Gứr. Voy. Favor. in Mag. Zool., 1838, p. 21.

Bords de la fossette mésosternale horizontaux; articles 3 et 4 des tarses munis en dessous d'une cupule arrondie.

abdominalis GuÉRIN; loc. cit.

Chili. rufiventris SolIER, loc. cit. 


\section{TRIBU XX.}

\section{ALLOTRMTES.}

Hanches postérieures à lame extérieure complète; articles 3 et 4 des tarses lamellés, au moins aux pattes antérieures.

\section{ALLOTRIUS.}

Casteln. Hist. nat. 1, p. 231.

Senodonia Cast. in Silb. Rev.

Forme parallèle; front très incliné; tarses à articles 1-4 lamellés.

quadricollis CASTELN, loc. cit. . . . . . J Java. sculpticollis FATRM. Ann. Fr., 1888, p. 350 . . Tonkin.

Parallotrius.

CAND. Élat. nouv. II, p. 45.

Pattes longues; articles 2-4 des tarses lobés.

pallipes Phwirpi (Hypodesis?) Ann. Univ.

Chile, 1861, p. 744 . . . . . . . . . . Chili.

Morostoma.

CAND. Cistula entom. II, 1879, p. 485.

Palpes à dernier article aussi longs que les antennes.

palpale CAND. loc. cit. . . . . . . Madagascar.

\section{HEMTOLIMERUS.}

Cand. Monogr. IV, p. 227.

Diffère des Allotrius par l'absence de lamelles aux premiers articles des tarses.

Emodi Cand. loc. cit. . . . . . . . . . Himalaya.

Gestroi Cand. Ann. Mus. Gênes, 1888, p. 685 . Birmanie. 
Penia.

Cast. Silb. Rev. entom. IV, p. 11.

Front peu incliné; élytres notablement plus larges que le prothorax, ordinairement élargies en arrière.

Eschscholtzi CAst. Silb. Rev. IV, p.11 . . N Népaul. soricina CAND. Monogr. IV, p. 230 . . . . Bornéo. birmanica CAND. Ann. Mus. Gênes, 1888, p. 685. Birmanie. fausta CAND. Ibid. . . . . . . . . . . ” lutea CAND. Ibid. . . . . . . . . . . ”

fulva CAND. Élat. nouv. I, p.55 . . . . . Java. stictica CANd. Not. Leyd. Mus., 1880, p. 3 . . Sumatra. hirtella CAND. Monogr. IV, p. 231 . . . . Himalaya. brevis CAND. Ibid. . . . . . . . . . . » longipes CAND. Ibid., p. 232 . . . . . . " tomentosa CAND. Ibid. . . . . . . . Hindoustan. nebrioides CAND. Ibid., p. 233 . . . . . Himalaya. leistoides CAND. Tbid., p. 234. . . . . . Hindoustan. canaliculata CAND. Ibid., p. 235 . . . . . » gracilis CAND. Ibid. . . . . . . . . . » laticornis Krrsch, Mitt. K. Zool. Mus. Dresd., 1875 . Malacca.

\section{TRIBU XXI.}

\section{DIMITES.}

Hanches postérieures très dilatées en dedans; limitées à une étroite lame'le, ou nulles, en dehors.

\section{Dina.}

Eschs. Silberm Rev. Entom. IV.

Celox Sohauf. Isis, 1863.

Quatrième arțicle des tarses lamellé; élytres très larges. 
elateroides Charp. Hor. Ent., p. 191 . . . . Styrie. dalmatina Kust. Käf. Eur. I, p. 13 . . . . Dalmatie. Perezi Seml. Berl. Ent. Zeitschr., 1867, p.178. Espagne. Assoi Perez, Ann. Soc. Espan. Sc. Nat., 1872, p. 131.

dima (s. nom. gen. Celox) Schauf., loc. cit.

\section{BELIOPHORUS.}

Escrs. Thon, Arch. I, II, p. 34.

Mésosternum horizontal; tarses simples.

cebrionoides Eschs., loc. cit.

\section{DiADYsis.}

CAND. Élat. nouv. II, p. 98.

Hanches postérieures réduites à une grande lame interne.

Morsi CAND. loc. cit., p.99. . . . . . . . Cap York.

Osorno.

CAND. Élat. nouv. III, p. 100.

Hanches postérieures brusquement dilatées en dedans.

ambiguus CAND. loc. cit. . . . . . . . Patagonie.

\section{TRIBU XXII.}

\section{HYPODÉSITES.}

Front légèrement bombé, fossettes antennaires grandes et apparentes: bouche inférieure; sutures prosternales courbes; hanches postérieures étroites; tarses trilamellés.

HYPODESIS.

Latr. Ann. Fr. II, p. 256.

sericea (Latr.) Germ. Zeitschr. I, p. 221. . . Mexique. 
chrysomalla CAND. Monogr. IV, p. 244. . . Mexique. penicillata CAND. Ibid. . . . . . . Honduras. punctata CAND. Tbid., p. 245 . . . . . . . ” cribricollis CAND. Ibid., p. 246 . . . . . . Mexique. vittata CAND. Ibid.

\section{TRIBU XXIII.}

\section{CARDIORHINITES.}

Front bombé; fossettes antennaires grandes; labre grand, sillonné, ce qui le fait paraître bilobé; tarses simples.

CARDioRHINUS.

Eschs. Thon, Arch. II, I, p. 34.

antennalis GerMr. Zeitschr. IV, p. 98.

Brésil.

ruflateris GERM. Tbid.

contaminatus GERM. Ins. Sp. nov.

axillaris? EscHs., loc. cit.

seminiger Eschs. Thon, Arch. I, II, p. 34 . . ” castaneipennis Germ. Zeitschr. IV, p. 95 . . ”

circumcinctus GERY. Ins. Sp. nov., p. 83 . . ”

bilineatus EscHs. Thon, Arch.

plebejus CAND. Monogr. IV, p. 258. . . . . ”

maculicollis CAND. Tbid., p. 259. . . . . . ”

plagiatus GERM. Ins. Sp. nov., p.51 . . . . . "

attenuatus Eschs. Thon, Arch.

brasiliensis CASTELN. Hist. Nat. Ins.

humeralis Eschs. Thon, Arch. II, p. 34 . . . "

vulneratus Gerir. Ins. Sp. nov., p. 56 . . . ”

mufilateris var. GERM. Zeitschr. IV.

sanguinolentus CAND. Monogr. IV, p. 264. . ”

frenatus GerM. Ins. Sp. nov., p. 53 . . . . ” 
acuminatus GERM. Ibid.

Brésil.

piciventris Germ. Zeitschr. IV, p. 98 . . . ”

simplex CAND. Monogr. IV, p. 267 . . . . . ”

modestus CAND. Ibid. . . . . . . . . Brésil mér.

lætipennis CAND. Élat. nouv. III, p. 102. . . ”

semirufus CAND. Monogr. [V, p. 268 . . . . Guyane.

hypocrita Erichs. Schomb. Guyana III, p.558. ”

æneolipennis CAND. Monogr. IV, p. 269. . . ’

vinulus CAND. Ibid., p. 270. . . . . . . . Para.

bilineatus FABR. System. Eleuth. $\amalg$, p. 235 . Guyane.

tactus CAND. Élat. nouv. III, p. 102 . . . . Amazones.

trivittatus CAND. Monogr. IV, p. 272. . . . Guyane.

cruentus CAND. Ibid., p. 273 . . . . . . . Brésil.

tæniatus CAND. Ibid., p. 274 . . . . . . . ”

bicolor CAND. Ibid. . . . . . . . . . . ”

inæqualis CAND. Ibid., p. 275 . . . . . . ”

cuneatus CAND. Ibid., p. 276 . . . . . . . ”

opacus CAND. Ibid. . . . . . . . . . . . . ”

bonariensis CAND. Ibid., p. 277. . . . . Buenos-Ayres.

basalis CAND. Ibid. . . . . . . . . . . Brésil.

pullatus ( CAND. Ibid., p. 276 . . . . . . . ”

pallidipennis CAND. Ibid., p. 279 . . . . . ”

sulcatus CAND. Ibid., p. 280 . . . . . Nouv. Grenade.

\section{TRIBU XXIV.}

\section{LUDITES.}

Front bombé; bouche inférieure; labre grand; mandibules perpendiculaires; sutures prosternales courbes.

Tomocephalus.

Latr. Ann. Fr., 1834, p. 146 (Emend.).

Megacnemius Eschs.

Mésosternum presque vertical; crêtes sus-antennaires très petites. 
sanguinicollis Latr, loc. cit.

Brésil.

sardioderus CAND. Monogr. IV, p. 286 . . . Merique.

substriatus CAND. Ibid. . . . . . . . Vénézuéla.

abdominalis CAND. Élat. nouv. IV, p.52 . . Véragua.

Probotriom.

CAND. Monogr. IV, p. 287.

Mésosternum vertical, le métasternum faisant saillie entre les jambes moyennes.

pubescens Krrb. Trans. Linn. Soc. 1818, p. $382 . \quad$ Brésil.

lampyrinus Perbr, Delect., p. 22.

velutinum GeRr. Zeitschr. $\nabla$, p. 184 . . . . ”

furvum Erichs. Wiegm. Arch., 1847, p. 78 . . Pérou.

rufipes CAND. Monogr. IV, p. 290 . . . . . Brésil.

Physorhinus CAND. Tbid. . . . . . . Mexique.

pupillum CAND. Ibid., p. 291 . . . . . N. Grenade.

gibbiferum CAND. Ibid., p. 272 . . . . . . Brésil.

rufivellum CAND. Ibid. . . . . . . . »

rufopubescens Stennн. Col. HefteXIV, p. 133. N. Grenade. crinitum CAND. Élat. nouv. II, p. 45 . . . . Quito. pilosum CANd. Tbid., p.46 . . . . . . . Bahia. amplicolle CAND. Monogr. IV, p. 293 . . . Para. setosum CANd. Tbid., p. 294 . . . . . . Colombie.

\section{Mecastrus.}

Sharp, Ann. a. Magaz. May 1877, p. 19.

Ludius à hanches postérieures peu dilatées en dedans. convexus Sharp, loc. cit. . . . . . . N. Zélande. discedens Sharp, Ibid. . . . . . . ” vicinus SHarp, Ibid. . . . . . . . . ”

LoDIUs.

Latr. Fam. Nat. du règne animal; 1825, p. 349. 
Aphanobius pars Gery. Zeitschr. V.

Steatoderus Escrs. Silberm. Rev. entom. IV.

Crigmus Lec. Trichophorus MuLs.

Amblygnathus, Genomecus Sor. Gay, Hist. Chil. Zool. V.

Front convexe, coupé obliquement de chaque côté, labre assez grand; antennes fortement dentées et même parfois pectinées chez les mâles, souvent hérissées de poils courts, angles postérieurs des angles prothoraciques munis d'un faisceau de poils au sommet.

\section{$1^{\text {er Groupe. }}$}

Espèces de l'ancien continent et îles voisines.

acutus CAND. Monogr. IV, p. 299 . . . . . Java.

ceylanicus CAND. Tbid., p. 300 . . . . . . Ceylan.

macassariensis CAND. Ibid. . . . . . Célèbes.

dissidens CAND. Ibid., p. 301. . . . . Ceylan.

aberrans KrRsch, Mitt. Zool. Dresd., 1875 . . Malacca.

illotipes CAND. Monogr. IV, p.302 . . . . Java.

rubiginosus CAND. Notes Leyd. Mus. XI, 1889, p. 96 . . . . . . . . . . . Sumatra.

suturalis CAND. Élat. nouv. IV, p. 53 . . . . Bornéo.

spissus CAND. Ibid. . . . . . . . . . . ”

lucidus CAND. Élat. nouv. I, p. 55 . . . . . Java.

hirtellus CAND. Monogr. IV, p. 303. . . . . Ceylan.

hirsutus CAND. Bull. Soc. entom. belg. xvHI . 1.Philippines.

Guillebeaui Murs. Opusc. entom. II, p.181. . Sicile.

Schaumi CAND. Élat. nouv. II, p. 103. . . . G Grèce.

tæygetanus Reitter, Deutsch. Entom. Zeits., 1887, p. 513. . . . . . . . . . ”

turanicus Reitter, Tbid. . . . . . . . Askabad.

Montandoni Buyss. Ann. Fr., 1888, bull. . . Bucharest.

ferrugineus LnNí, System. Nat. ed. 10, p.405. Europe.

v. occitanus Villers, Entom. I.

luctuosus Solsk. Hor. Soc. ent. Ross., 1871. . Vladivostock. 
sericans CAND. Élat. nouv. I, p. 55.

Ceylan.

Sieboldti Cand. Mém. Soc. Sc. Liége $\mathrm{V}, 2^{\mathrm{e}}$ sér.

Japon.

sinensis CAND. Élat. nouv. III, p. 103. . . . Chine.

sylheticus CAND. Idid.

Sihlet.

ligatus (pour lineatus) Cand. Mém. Soc. Sc.

Liége $V$.

Japon.

junior CAND. Ibid.

plebejus CAND. Ibid.

anchastinus CAND. Élat. nouv. III, p. 104.

grandis FALD. Bull. Moscou LX, 1836, p. 368 . Turcomanie. penicillatus Gerst. Beitr. Insekt. Zanz., 1871,

p. 54 .

Zanzibar.

\section{2e Goupe.}

Nouveau continent.

attenuatus $\mathrm{S}_{A X}$, Trans. Am. Soc. VI .

États-Unis.

fuscus Cast. Hist. Nat. Ins. I, p. 240.

Lecontei Horn, Tr. Entom. Soc. Am., 1874. ater CAND. Élat. nouv. I, p. 55.

Californie.

tartareus Lec. Proc. Acad. Phil., 1859, p. 85.

aterrimus Moтsch. Bull. Mosc. 1859.

pinguis Hors,Tr. Entom. Soc. Am., 1874

Amér. bor.

abruptus $\mathrm{S}_{A \mathrm{Y}}$, Ann. Lyc. I, p. 253.

)

coracinus GerMr. Zeitschr. IV, p. 47.

texanus Lec. Trans. Am. phil. Soc. X, p. 454. hepaticus GERMr. Ins. Sp. nov., p. 43 .

Texas. limbalis HerBst, Käf. IX, p. 53.

États-Unis.

havaniensis CAND. Monogr. IV, p. 309

Cuba.

brevis CAND. Élat. ṇouv. II, p. 46

Brésil.

subsericeus CAND. Monogr. IV, p. 309

Mexique.

crassus CAND. Tbid., p. 308

Cayenne.

brunnipilis CAND. Ibid., p. 310 . . . . . Nouv. Grenade.

decorus Germ. Zeitschr. IV, p. 48 .

Chili.

granulosus Sor. GAY. Hist. Chile V, p. 32. 
ruficollis Sol. GAY, Histor. Chile V, p. 30 .

Chili.

abdominalis SoL. Tbid.

ruficornis PншірPI, Ann. Univ. Chile, 1861 • ”

corralensis PHшIPPI, Ibid. . . . . . . . »

3 Groupe.

Australie, NHe Guinée et îles voisines.

exutus CAND. Monogr. IV, p. 304 . . . . . Australie. lineatus CAND. Ibid. . . . . . . . . . ”

hydropicus CAND. Élat. nouv. III, p. 104 . . Queensland.

atripennis MAC LeAY, Tr. N. S. Wal. II, p. 259.

variegatus CAND. Ann.Mus. Gênes XII, p.138. Arou.

erubescens CAND. Élat. nouv. II, p. 46 . . Nouv. Grinée.

dilaticollis Fatrir. Ann. Soc. Entom. belg.,

1883 . . . . . . . . . . . Nouv. Hébrides.

ORTHOSTETHUS.

LACord. Gener. des Col. IV, p. 217.

Aphanobius Germ. Zeitschr. V, p. 183.

Mésosternum horizontal; hanches larges en dehors.

infuscatus GerMr. Zeitschr. V, p. 183 . . . Etats-Unis mér. sordidus MeLsh. Proc. Ac. Phil. II, p. 216.

piceus CAND. Monogr. IV, p. 315 . . . . Mexique. præfectus CAND. Ibid. . . . . . . . . Brésil. corvinus Germ. Zeitschr. $\nabla$, p. 183 . . . . Nouv. Grenade. Landoldti Stennн. Mitt. d. Munch. Ver., 1877, 84. ”

\section{ApHanobius.}

Eschs. Thon, Arch. I, II, p. 33.

Mésosternum incliné; hanches postérieures larges en dehors.

alaomorphus CAND. Monogr. IV, p. 319. . . Indo-Chine. malaccensis CAND. Ibid. . . . . . . . Singapore. cylindricus CAND. Tbid., p.320 . . . . . Camboge. 
longithorax WIEDM. Zool. Mag. II, I, p. 106 .

Bengate. æqualis CAND. Élat. nouv. II, p. 46 .

Siam. gracilis CAND. Élat. nouv. IV, p.53 . . . Cochinchine. stenosomus CAND. Monogr. IV, p. 320 . . . Java. discoidalis CAND. Ann. Mus. Gênes, 1880, p. 197. . . . . . . . . Sumatra. longus CAND. Monogr. IV, p. 322 . . . . I. Plitippines. thoracicus CAND. Élat. nouv. IV, p. 53 . . . Zanguebar. seclusus CAND. Monogr. IV, p. 31 . . . . . Calabar. acutipennis Germ. Zeitschr. V, p.187 . . . 1. Maurice. vanus GerMr. Tbid. . . . . . . . . . . » lucius CAND. Monogr. IV, p.324. . . . . . ” bistrigatus CAND. Tbid. . . . . . . . . »

LODIGENUS.

CAND. Monogr. IV, 1863, p. 325.

Mésosternum horizontal; hanches larges en dedans, très amincies en dehors.

politus CAND. loc. cit. . . . . . Indo-Chine, Malaisie. primævus CAND. Ibid. . . . . . . . . . ”

\section{Dioxypterds.}

Fatru. Ann. Fr., 1881, p. 267.

Front plus allongé que chez les Aphanobius, corps très cambré. nigrotransversus FAIRM. loc. cit. . . . . Viti. flexuosus Fatrm. Ibid. . . . . . . . . ” guttulatus FATRM. Ibid. . . . . . . ” variegatus FAtrм. Ibid. . . . . . . . . . »

\section{Scelisus.}

CAND. Monogr. IV, 1863, p. 327.

Sutures prosternales canaliculées dans presque toute leur longueur. sanguineus CAND. loc. cit. . .. . . . . Népaul. 


\section{Paranilicus.}

CAND. Élat. nouv. II, p. 47.

Ophidius Mac Leap.

Front acuminé; hanches postérieures peu dilatées en dedans.

brevicornis Mac LeaY, Tr. N. S. W. II, p.254. Queensland. Mac Leayi CAND. Élat. nouv. II, p. 47 . . . Victoria.

\section{ANmicus.}

Cand. Monogr. IV, p. 328.

Ampedus Germ. Melanoxanthus Erichs.

Antennes courtes, épaisses; hanches postérieures dilatées dans leur moitié interne.

semiflavus Germ. Zeitschr. V, p. 163 . . . . Australie. attenuatus CAND. Monogr. IV, p. 330 . . . "

loricatus CAND. Ibid. . . . . . . . . . Australie occ. quadriguttatus Ericris. Wiegm. Arch., 1842,

p. 139. . . . . . . . . . . Tasmanie. flavipennis CAND. Élat. nouv. II, p. 47. . . . Melbourne. hæmorrhoidalis CAND. Ibid. IV, p. 54 . . . - » nigroterminatus Mac LeAY, King Survey, II, p.441. . . . . . . . . . . Australie.

COMPSHELUS.

CAND. Élat. nouv. II, p. 48.

Tarses légèrement dilatés, poilus en dessous.

flavus CAND. loc. cit. . . . . . . . . I. Viti.

\section{Monelasmus.}

Cand. Monogr. IV, p. 332.

Tarses à troisième article lamellé.

Augusti Cand. loc. cit., p. 333. . . . . . Mexique. 
moratus CAND. Ibid., p. 334

Mexique.

Jamaicæ CAND. Ibid. . . . . . . . J Jamaïque.

insularis CAND. Elat. nouv. IV, p. 54 . . . . Martinique.

guyanensis Caxd. Monogr. IV, p. 335. . . . Guyane.

Agelasinus.

CAND. Monogr. IV, p. 335.

Fossettes antennaires nulles.

viridis CAND. loc. cit. . . . . . . . . Grenade. limbatipennis Sternh. Col. Hefte XIV, p. 133. suturalis Sтепн. Ibid. . . . . . . ” metallescens Sternн. Ibid. . . . . . . ” campyloides CAND. Monogr. IV, p. 337 . . . »

\section{Cosmesus.}

Eschs. Thon, Arch. I, II, p. 33.

Corps allongé, svelte; à facies de Pumachilius; à front bombé et sans rebord; tarses simples.

\section{section 1.}

Elytres distinctement échancrées au sommet.

bilineatus Eschs. loc. cit. . . . . . . .

Brésil.

unilineatus CAND. Monogr. IV, p. $3+1$.

sexguttatus CAND. Ibid., p. 342 .

sexpustulatus CAND. Ibid., p. 343

guttatus CAND. Ibid., p. 344

posticus CAND. Ibid.

obliquus CAND. Ibid., p. 345

quadrimaculatus CAND. Ibid.

figuratus CAND. Ibid., p. 346 .

discoidalis Kırsen, Berl. Ent. Zeit., 1870,

p. 375.

Bogota

humeralis CAxd. Monogr. IV. p. 346 .

Brésil. 
lineatocollis CAND. Ibid., p. 347. . . . . . Brésil.

obscurofasciatus CAND. Tbid., p. 348 . . . ”

brunneus CAND. Ibid. . . . . . . . . ”

apicatus CAND. Ibid., p. 349 . . . . . . . . ”

fasciatus CAND. Ibid. . . . . . . . . . ”

gracilis KrnscH, Berl. Entom. Zeitschr., 1873,

p. 369 . . . . . . . . . . . Pérou.

striatus CAND. Monogr. IV, p. 350 . . . . Chili.

lucidus CAND. Ibid. . . . . . . Nouv. Grenade.

electus CAND. Tbid., p. 351 . . . . . . . . ”

\section{Bection II.}

Élytres entières ou peu distinctement tronquées au bout.

punctum CAND. Élat. nouv. IV, p. 54 . . . Équateur. monachus CAND. Monogr. IV, p. 352 . . . Brésil. suturalis SteInh. Col. Hefte XIV, p. 135 . Nouv. Grenade. depressus Steinн. Tbid. . . . . . . . . ” bonariensis BoHer. Eugen. Resa, p. 69 . . Buenos-Ayres. obtusipennis Sternh. Atti d. Soc. Ital. Sc. Nat., 1873, p. 569.

minor CAND. Monogr. IV, p. 353

Brésil. marginatus CAND. Tbid. cinctus CAND. Ibid., p. 354. . . . . . . . ” pictus CAND. Tbid. subtilis CAND. Tbid., p. 355. rugatus $\mathrm{C}_{\triangle \mathrm{ND}}$. Tbid. sulcifrons CAND. 1bid., p. 356. flaveolus $\mathrm{K}_{\mathrm{IRSCH}}$, Berl. Entom. Zeitsch., 1870,

p. 375 . Bogota. flavidus CAND. Monogr. IV, p. 356. . . . Caracas. pallidulus CAND. Tbid,, p. 357. . . . . . . Callao. brevis CAND. Ibid. . . . . . . . . Nouv. Grenade. maurus CAND. Ibid., p. 358 . . . . . . . ” nigrans CAND. Élat. nouv. IV, p. 54. . . . . Équateur. ater Stenn. Col. Hefte XIV, p.134 . . . Nouv. Grenade. 
Agriotes.

Eschs. Thon, Arch. I, II, p. 34.

Ectinus Eschs., Curtis, Redt. - Cataphagus StepH.

Dolopius MeLsh., Lec., etc. Synaptus FALD.

Adrastus pars LACord.

Sutures latérales du prothorax infléchies en avant, coloration générale brune et uniforme (sauf les espèces du Mexique); correspond aux Cosmesus de l'Amérique méridionale et aux Agonischius de l'Inde.

\section{Ier Groupe.}

Ancien continent (1).

aterrimus LinN. Syst. Nat., 1, 2, p. 653 . . . Europe. atratus ImLig. Mag. IV, p. 100.

niger De Geer, Mém. Ins. IV, p. 151.

จ. volhiniensis FIscH. Entom. Ross. II, p. 202.

gurgistanus Falderm. Faun. Transc. I, p. 161. Asie min. turcicus CAND. Monogr. IV, p. 379. . . . . Turquie. corsicus CAND. Ibid. . . . . . . . . . Corse.

Desbrochersi Buyss. Ann. Fr. 1890, Bullet, octobre . . . . . . . . .. . Algérie. pilosellus Schöкh. Syn. Ins. 1, 3, p. 307 . . . Europe. elongatus Marsh. Entom. Brit, I. pilosus $\mathrm{Pz}$. Entom. Tasch. CANd. Monogr. IV. vilis ILurg. Mag. VI, p. 5.

v. nudus Küster, Käf. Eur., p. 27, 84.

nuceus Fatrar. Ann. Fr., 1866, p. 260 . . . Asie mineure. Grandini CAND. Monogr. IV, p. 381 . . . . Algérie. tauricus HeYD. Deutsch. Entom. Zeitschr., 1881, p. 155. . . . . . . . . Crimée.

(1) M. 0. Schwarz, bibliothécaire de la Société entomologique allemande, à Berlin, soccupe actuellement d'une révision des duriotes de la faune paléarctique. Je Jois à son obligeance de précieux renseignements sur la validité de plusieurs espèces qui m'étaient inconnues. 
caspicus Herd. Ibid., 1882, p. 358 . . . . . Turcomanie. piceus Ménétr. Mém. Acad. Pétersb. VI, p. 51. ” unicolor KöNIG, Hor. Soc. Ross., 1889. Turkestan chinois. Laichartingi GreDL. Käf. Tyrol., 1863,1, p. 219. Tyrol. meridionalis, attenuatus Desbr. Abeille, 1869, p. 119.

ustulatus Schatl. Schr. Ges. Hall., 1783, I, p. 303. Europe. blandus Germ. Ins. Sp. nov., p. 4. confusus BACH, Kaferf. III, p. 47. flavicornis REDT. Fn. Austr., p. 509. gilvellus Lac. Fn. Par., p. 670. ustulus ScHöNH. Syn. Ins. variabilis HERBst, Käf. $\mathrm{X}$.

sputator LINNé, Syst. Nat. $10^{\circ}$ éd., p. 405 . . ” fusculus Luisg. Mag. 4, p. 101. brunnicollis GEBL. Ledeb. Reis., p. 80. graminicola Redt. Faun. Austr. cribrosus, corallifer Eschs. Die Quatem., 1830. lapicida FALD. Faun. transc.; p. 177 .

Perse. hispanicus Dessr. Mitth. Schw. Ges. III . . Espagne. lineatus Linn. System. Nat. 1, 2, p.653 . . . Europe. segetis BJERK. Vet. Ak. Nya Handl., 1779. striatus, striolatus $\mathrm{F}_{\mathrm{ABR}}$. var. strigosus KIEs. Nat. Ins. IV, 2, p. 261. obscurus LinNé, System. Nat. 1, 2, p. 655 . . . ” badius Mour. Zool. Dan. Pr., p. 60. hirtellus Herbst, Käf. X, p. 94. obtusus De GeER, Mém. Ins. IV, p. 147. variabilis FABR. Entom. Syst. 1, 2, p. 230.

rubiginosus CAND. Monogr. IV, p. 389 . . . Corfou. marginipennis LUCAs, Expl. Alg., p. 168 . . Algérie. brevis CAND. Monogr.IV, p. 390. . . . . . France mér. sordidus ILLig. Mag. VI, p. 7 . . . . . . Europe mér. rufipalpis Brulle, Exp. Mor., p. 137. curtus CAND. Élat. nouv. II, p. 49 . . . . . Maroc. 
italicus Band. Berl. Ent. Zeits., 1871, p. 55 . . Italie. punctulatus Brullé, Exp. Mor. Zool., p. 142. Grèce. flavobasalis Heyd. Deutsch. entom. Zeits., 1889, p. 38

Portugal. nitidicollis MrLu. Ibid., 1881, p. 220 Bude. paludum Kies. Berl. Zeits., 1859, p. 18. Grèce. litigiosus Rossr, Mant. Ins., 1792, p. 58 . Italie.

italicus BaUdr, Berl. Entom. Zeits., 1871, p. 55 .

Bonnairei Buyss. Ann. Fr., 1889, Bull., p. 214. dahuricus CAND. Monogr. IV, p. 394 . meticulosus CAND. Ibid., p. 396.

Oran. castaneus FATRM. Ann. Fr., 1852, p. 81 Daourie. Syrie. Sicile. modestus Kies. Nat. Ins. IV, 2, p. 259 Hongrie. fliformis CAND. Monogr. IV, p. 397 Turquie. sobrinus Kres. Nat. Ins. IV, 2, p. 258. Europe.

pallidulus ReDt. Fn. Austr., p. 511. pallidulus Illig. Mag. VI, p. 6 . .

umbrinus Gerr. Ins. Sp. nov., p. 46. gallicus Lac. Fn. Par. I, p. 670 piceolus Kust. Käf. Europ. 27, p. 77.

Dalmatie. monachus Muls. Opusc. Entom. VI, p. 165. tauricus HEYD. Deutsch. Ent. Zeits. XXVI, 1882 , p. 155.

Crimée. murinus Mrut. Wien. Ent. Mon, 1862, p. 343. I. Ioniennes. subvittatus M[oтscH. Bull. Mosc., 1859, IV, p. 490 .

karabachensis Kolex. Meletem. V, p. 39. fusiformis CAND. Elat. nouv. II, p. 49. Caucase. exulatus Cand. Mém. Soc. Liége V, $2^{`}$ sér . Cline. leucophæatus CAND. Ibid. Japon. helvolus CAND. Ibid. sericeus CAND. Élat. nouv. II, p. 49 
Gandezei Lewis Cat. (pour ferrugineipennis

Mots. Bull. M., $1866\left({ }^{(}\right)$. . . . . . . . Japon.

sericans Lewis, Monthl. Mag. Déc. 1879. . . ”

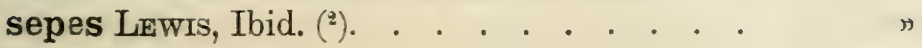

2e Grougr.

Youveau continent.

mancus SAY, Journ. Ac. Phil. III, p. 171. . . Missouri. obesus HarRIS, Ins. injur. Veget. I, p. 49.

striatulus, truncatus Melsh. Pr. Ac. Phil. II, p. 217.

stabilis Lec. Trans. Am. Ph. Soc. X, p. 457. insanus CAND. Monogr. IV, p. 376.

Canada. sordidus LEC. loc. cit.

fucosus Lec. Trans. Am. Soc. X, p. 456 . . Canada. var. collaris LEc, loc. cit.

ferrugineip snni ; LEc. Proc. Ac. Phil., 1861, p. 348.

imperfectus LEC. Trans. Entom.Soc.Am., 1884. sparsus Lec. Ibid. . . . . . . . . . . pubescens Melsh. Proc. Acad. Phil. II, p. 217. limosus Lec. Trans. Am. phil. Soc., p. 457 . . hispidus Lec. Trans. entom. Soc., 188t . . opaculus Lec. Proc. Acad. Phil., 1859, p. 85 . oblongicollis MeLSH. Ibid. II, p. 218 .

var. isabellinus MELSH., loc. cit.

nevadensis Lec. Trans. Am. ent. Soc., 1884 . Nevada. apicalis Lec. Ibid. . . . . . . . . . . Californie. avulsus Lec. Trans. Am. phil. Soc. X, p. 457 . Élats-Unis. torquatus Lec. Trans. Am. ent. Soc., 1884 . . Californie. montanus LEc. Ibid. . . . . . . . . Wyoming.

(1) Noms employés antérieurement (1861) par Leconte.

() Duxquets il faut ajouter les suivants dont la valeur spécifịue parait douteuse: Acuminatus Stepli. Infuscatus (Mingrélie) Olivievi (Algérie) DEsbr. 
inversus CAND. Monogr. IV, p. 375.

Californie.

Sericosomus flavipennis Mots.? (Lec., HorN.)

pulcherrimus CAND. Ibid., p. 364.

Mexique.

tæniatus CAND. Ibid.

ligatus $\mathrm{C}_{\mathrm{AND}}$. Élat. nouv. II, p. 48 . Guatémala.

pexus CAND. Monogr. IV, p. 365 .

))

fulvescens CAND. Ibid.

amabilis CAND. Ibid., p. 366

Mexique.

quadrivittatus CAND. Ibid.

notatus CAND. Ibid., p. 367.

virgatus $\mathrm{C}_{\mathrm{AND}}$. Ibid., p. 368 .

miniaticollis CAND. Ibid., p. 369.

hilaris $\mathrm{CAND}_{\mathrm{AND}}$. Ibid.

bicolor CAND. Ibid., p. 370.

lineipennis CAND. Ibid., p. 371

alternus CAND. Tbid.

longipennis. CAND. Ibid.

vaccinus CAND. Ibid., p. 372

guadulpensis $\mathrm{C}_{A \mathrm{ND}}$. Ibid.

)

lateralis CAND. Élat. nouv. II, p. 48

)

)

Guadeloupe.

australis FAIRM. Ann. Fr., 1883. . . . . Punta Aréna.

\section{Oxygonds.}

LEc. List. Col. North. Am., 1863, p. 48.

obesus SAY, Journ. Ac. Phil. III, p. 168

Missouvi.

acutipennis RaNd. Bost. Journ. II, p. 36.

ater Hons, Trans. Am. Soc., 1871, p. 318. . . Californie.

\section{Leptoschema.}

Hors, Trans. Am. Ent. Soc., 1884.

protractum HonN (Agriotes), loc. cit., 1871,

p. 227.

Californie. 
bicolor LEC. (Athous) Trans. Am. phil. Soc. X,

p. 428. . . . . . . . . . . . . . New-York.

discalceatum $\mathrm{S}_{A Y}$ (Elater), Ibid.IV, p.169. New-Hampshire.

Agonischios.

CAND. Monogr. IV, p. 407.

Représentent les Agriotes dans l'Inde; généralement parés de couleurs brillantes.

pectoralis CAND., Monogr. IV, p.411. . . . Bornéo. sanguinipennis CAND. Ibid. . . . . . . Java. fusiformis CAND. Mém. Soc. Ent. Belg., Bull., 1875

Mindanao. mirus CAND. Monogr. IV, p.412. . . . . . Indes or. Wallacei CAND. Ibid. . . . . . . . . . Bornéo. cyaneus CAND. Ibid. . . . . . . . . . Chusan. cyanipennis CAND. Ibid., p. 418. . . . . . Thibet. militaris CAND. Élat. nouv. III, p. 104. . . . Andamans. scutellaris CAND. Monogr. IV, p.413. . . . Singhapore. ornatus CAnd. Notes Leyd. Mus. II, p. 10 . Nouv. Guinée. gemmula CAND. Élat. nouv. II, p. 50 . . . . Hong-Kong. pantolamprus CAND. Monogr. IV, p. 419 . . Himalaya. altus CAND. Élat. nouv. IV, p. 54. . . . . . Darjeeling. thoracicus Freut. Ann. Fr., 1889 . . . . . Camboge. annamensis CAND. Monogr. IV, p. 413 . . . Cochinchine. conjungens CAND. Ibid. . . . . . . Singhapore. virgulatus CAND. Élat. nouv. IV, p. 55 . . . Bornéo. brevicollis CaND. Bull. Soc. Entom. xṿI, p. 127.

Philippines. basalis CAND. Ibid. ) Gastelnaui CAND. Monogr. IV, p. 417. . . . Siam. cyaneus CAND. Ibid., p. 418 . . . . . . . Chusan. æneolus CAND. Ibid, p. 417 . . . . . . J Java. lepidus CAND. Ibid., p. 415. . . . . . . . Cochinchine. Delaunayi Fleut. Ann. Fr., 1889 . . . . Annam. 
dorsalis CAND. Elat. nouv. II, p. 50

Canton. cyanopterus CAND. Ibid.

Madras.

bitinctus CAND. Monogr. IV, p. 416

Camboge.

suturalis CAND. Ibid.

)

unicolor CAND. Élat. nouv. II, p. 51.

)

Feæ Cand. Ann. Mus. Gênes, 1888, p. 688

Tenassérim.

cinctus CAND. Iilat. nouv. II, p. 49.

Bengale.

Cardoni Cand. Bull. Soc. Ent. Belg., 1890

)

bimaculatus C.xp. Notes Leyd. Mus. II, 1880.

Sumatra.

obscuripes Grut. Schönh. Syn. Ins. App.,

p. 131.

Chine.

caschmirensis Kollan, Hügel Caschm.IV,p.507.

viridis CAND. Monogr. IV, p. 421

Camboge. cineraceus $\mathrm{C}_{\mathrm{AND}}$. Ibid., p. 422

Singleapore.

cinnamomeus CAND. Ibid., p. 420 .

Himalaya.

semiluteus CAND. Elat. nouv. IV, p. 55

Bornéo.

chalcoxanthus CAND. Monogr., p. 415

Camboge.

luteus CAND. Tbid., p. 422

Java.

quadrilineatus Hope (Ludius), Trans. Ent.

Soc. Lond. IV, p. 10.

Cline.

sulcicollis CAXD. Élat. nouv. II, p. 50.

Sicum.

monachus CAND. Ibid., p. 51 .

Canton.

fasciatus CAND. Notes Leyd. Mus., 1880.

Sumatra.

lateralis CAND. Tbid.

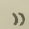

montanus Cavd. Ann. Mus. Gênes, 1880, p.197.

conspurcatus CAND. Notes Leyd. Mus. V,p.213

speculifer Fatrm. Ann. Fr., 1889, p. 34 .

Chine. religiosus CAND. Élat. nouv. IV, p. 55 . . . Cochinchine. decoratus CAND. Ibid.

)

frenatus CAND. Ibid. II, p.51. . . . . . . Ceylan. prymneus CAND. Monogr. IV, p. 42t . . Bengale, Ceylan. cardiorhinulus CAND. Ibid. . . . . . . Ceylan. finitimus CAnd. Bull. Soc. Entom. Belg., 1890. Bengale. æneipennis Ktrsch, Mitt. Mus. Zool. Dresd., 1875, p. 9

Malacca. 
chalcopterus CAND. Élat. nouv. II, p. 50. . . Madras.

Lansbergei CAND. Ibid.p. 127 . . . . . Billiton. marginatus CAND. Bull. Soc. Ent. Belg. 1875, Mindanao.

t. XVII, p. 117.

scapularis CAND. Monogr. IV, p. 425. . . . Hong-Kong. vittiger HEYD. Hor. Soc. Ross., 1888, p. 262 . Coree. subsericeus Kolв. Arch. Sc. Nat., 1887, p.197. breviusculus CAND. Monogr. IV, p. 426. . . Hong-Kong. apicalis (Dicteniophorus) MaC Leay, Tr. N. S.

W. II, p. 26. vittatus $(i d$.$) Mac Leay, Tbid. . . . . . . "$ ventosus CAND. Élat. nouv. IV, p.56 . . . Port Denison. longicornis CAND. Élat. nouv. III, p. 105 . . Queensland. australis CAND. Ibid. $\Pi$, p.51. . . . . . . ” semiflavus CAND. Notes Leyd. Mus., 1887,

p. 288. . . . . . . . . . . Normantown. brevis CAND. Tbid. lineatus CAND. Élat. nouv. II, p. 51. . . . . Australie.

\section{SERICUS.}

Eschs. Thon, Arch. I, II, p. 34. Atractopterus Lec. Dolopius Eschs. (pars).

Ectinus Redt. .ericosomus Steph., Lacord., Cand.

Caractères des Agriotes, sauf les bords du corselet dont la suture n'est pas infléchie au sommet.

brunneus Lrvv. Syst. Nat. éd. 10, p. 404 . . Europe. fugax GrLL. Ins. suec. I, p. 428.

tibialis Redt. Fn. Austr. $2^{e}$ éd., p. 510. vulgaris Herbst, nigromaculatus De GeER.

subæneus W. REDT. Quæd. Gen. et Sp., p. 12. ) jucundus, xanthodon, MärkeL, Stett. Zeit., 1847. micans Muls. Opusc. entom. VI. puerilis CANd. Deuts. Ent. Zeitschr., 1879, p. 282. Amur. 
affinis Мотьсн. Bull. Acad. Pét. 1859, p. 544 . Jukutsli. contiguus FALD. Fn. transc. I, p. 178 . . . . Caucase. sericarius Мотьсн. Bull. Mosc., 1866, p. 166 . Japon. marginatus Lins. Syst. Nat, éd. 10, p. 405. . Europe. lateralis Ou. Entom. sticticus Panz. Fn.

fulvus, suturalis MARSH. Ent. brit.

depressus Eschs. Mém. Ac. Pét. 1818.

lateralis Eschs. Thon, Arch. II, I, p. 3t. . . Amér. bor. californicus, sellatus Mannerh. B. M. 1852. pauper, macer, subustus, LEc. Trans. X.

Theveneti Hors, Trans. am. ent. Society 1872, p. 148 .

Californie. fusiformis Lec. Trans. Am. ph. Soc. X, p. 45t. Canada. viridanus SAY, Ann. Lyc. I, p. 250. . . . Amér. bor. sublucens RaND. Bost. Journ. II, p. 37.

incongruus Lec. loc. cit. . . . . . . . Lac Supérieur. silaceus SAY, Ann. Lyc. I, p. 260. ํ. . . Amér. bor. v. debilis Lec. Proc. Ac. Phil., 1859, p. 72.

ơ umbraticus Lec. Trans. X, p. 505.

Behrensi (') CANd. Elat. nouv. IV, p. 56 . . . Californie.

\section{Pittonotus.}

Kies. Sch. cat., 1862, p. 58.

Ectinus CAND.

theseus Germ. Reis. Dalm., p. 258 . Europe or.

Simoni Stierd. Mitt. Schw. Ent. Ges. V, p. 511.

DoLOPORUS.

Cand. Notes Leyd. Mus. IX, p. 289.

Front large, bombé; tarses à articles 1-3 un peu dilatés. aterrimus CAND. loc. cit. . . . . . . Normantown. thoracicus CAND. Tbid.

(') Les suivants, de Motschoulski, me sont inconnus : basalis (l'eus.) humeralis, simplex, sericatus (Cal.). 
ACRONIOPUS.

Erichs. Wiegm. Arch., 1843, II, p. 175.

Atelopus pars, ERICHs.

Front d'Agriotes; les tarses à 40 article lamellé.

fuliginosus CAND. Monogr. IV, p. 438 . . . Sidney.

ater CAND. Tbid.

humilis ERICHs. loc. cit., 1842, I, p. 143 . . . Tasmanie.

infimus Erichs. Ibid. . . . . . . . . . »

pubescens Mac Leax, N. S. W.Trans. II, p. 250. Queensland. rufipennis MAC LeaY, Ibid. . . . . . . . ”

sidneyanus BoIsD. Voy. Astrol. Col. 1835, p. 109. Sidney. grandis REDT. Reis Novarra II, p. 96. . . . N. Zélande.

Ascesis.

CAND. Monogr. IV, p. 440.

Facies, antennes et hanches postérieures des Monocrepidius australiens avec une tête de Ludiite.

australis CAND. loc. cit. . . . . . . . Australie or.

DICTENIOPHORUS.

CAND. Monogr. IV, p. 441.

Ludius Eschs. Corymbites Germ.

Antennes pectinées, chez les mâles, à partir du $33^{\ominus}$ article; dentées chez les femelles.

ramifer Eschs. Thon Arch. I, II; p. 34. . . . Australie. Hunteri Borsd. Fn. Océan.

badiipennis CAND. Monogr. IV, p. 443 . . . ”

fusiformis CAND. Ibid. . . . . . . . . Australie sept.

melanoderus CAND. Ibid. . . . . . . Melbourne.

vitticollis MAC Leay, N. S. W. Trans. II, p.260. Queensland.

Ochosternus.

CAND. Monogr. IV, p. 445.

Nycterilampus Montr. Ann. Fr., 1860, p. 258. 
Antennes pectinées chez les mâles à partir du $4^{\circ}$ article. zealandicus White. Voy. Ereb. a. Ter., p. 7. Nouv.Zélande. ơ punctithorax White. Ibid. Parryi CAND. Monogr. IV, p. 447 . . . . . » lifuanus Montr., loc. cit. . . . . . . Nouv. Calédonie. gigas CAND. Élat. nouv. III, p. 105 . . . . . 》 》

\section{Thoramus (').}

Sharp, Ann. a: Mag., May 1877.

Wakefieldi Sharp, loc. cit., p. 5. . . . . Nouv. Zélande. obscurus Sharp, Ibid.

Feredayi Scharp, Ibid.

angustus Broun, Man. New Z. Col., 1881, p.678. ”

parvulus Broun, Ibid. . . . . . . . . "

cervinus Broun, Ibid. ....... ”

Huttoni Scharp, Ann. a. Mag., 1887, p.400. . ”

Geranus.

SHArp, Ann. a. Mag., May 1877.

lineicollis White. Voy. Er. a. Terr. - . Nouv. Zélande.

† fulvus SHARP, loc. cit.

crassus Sharp, Ibid. . . . . . . . . . ”

similis SHARP, Ibid. . . . . . . . . . "

collaris Pasc. Ann. a. Mag., 1876 . . . . . "

nitidofuscus BL. Voy. Pol. Sud IV, p. 88 . . ”

\section{TRIBU XXV.}

\section{ADRASTITES.}

Front bombé, sans carène, les crêtes sus-antennaires obliques; la bouche inférieure; crochets des tarses pectinés.

(') Ce genre et le précélent ne paraissent pas trìs distincts, mais je n'ai pas les éléments nécessaires pour élucider celte question. 


\section{Synaptus.}

Ctenonychus Steph. Cand. Monogr.

Article 3 des tarses lamellé, le 4 mo petit, simple.

filiformis FABR. Entom. Syst. II, p. 227 . . . Europe. incola HeRBst, Käf. X.

unguliserris SснӧNн. Syn. Ins. App.

cinereus IuLIG. Mag. VI.

hirsutus STEPH. Illustr. Brit. Entom. III.

v. erivanus FALD. Faun. Transc. I.

\section{GLYPHONYx.}

CAND. Monogr. IV, p. 451.

Adrastus pars ERICHs. Zeitschr. III.

Ctenonychus QuorumD.

Tarses à $4^{e}$ article lamellé, le cinquième court; front acuminé au-dessus de l'insertion du labre.

\section{Groupe.}

Espèces d'Amérique.

prævius ERICHs. Zeitschr. III, p. 121 . . . St-Domingue. marmorosus (PAL.) DeJ. Cat.

bivittatus CAND. Monogr. IV, p. 455 . . . . ”

Gundlachi Cand. Ibid. . . . . . . . . Cuba.

scabriusculus Chev. (Silesis) Ann. Fr., 1867,

p. 614. . . . . . . . . . . . . . . 》

fusculus Erichs. Zeitschr. III, p. 119. . . . » recticollis SAY, Journ. Acad. Phil. III, p. 168. Etats-Unis. inscius SAY, Trans. Am. Phil. VI.

pumilus Erichs. Zeitschr. III.

testaceus Mersh. Proc. Acad. Phil. II, p. 219.

)

quietus SAY, Trans. Am. Phil. VI, p. 184 . . Missouri. inquinatus $S_{A Y}$ Ibid. . . . . . . . . . Caroline $N$. 
mimeticus Hors, Trans. Am. phil. Soc., 1874,

p. 23 .

Caroline $N$.

nigritus CAxD. Élat. nouv. II, p. 52 . . . . Mexique.

cruciellus Erichs. Zeitschr. III, p. 120 . . . Colombie.

niger Steinh. Col. Hefte XIV, p. 136 . . - Noun. Grenade.

suturellus StennH. Ibid. . . . . . . . . »

antiquus CAND. Élat. nouv. III, p. 108 . . . »

se Goupre.

Espèces de l'ancien continent.

semipunctatus CAND. Élat. nouv. IV, p.56. . Bengale. brunneus CAND. Ibid. II, p. 52 . . . . . . Birmanie

flavidus CAND. Monogr. IV, p. 458 . . . . . Ceylan.

illepidus CAND. Mém. Liége, $2^{e}$ sér. V. . . Japon.

aberrans CANd. Monogr. IV, p. $456^{\bullet}$. . . . Boméo.

rubricus CAND. Ibid. . . . . . . . .

pallidulus CAND. Ibid. . . . . . . . . . . "

zonatus CAxD. Ann. Mus. Gênes, XII, p. 139 . ”

dorsalis CAỸ. Iloid. . . . . . . . Jar

quadrimaculatus CAND. Ibid. . . . . . ”

frontalis CAND. Notes Leyd. Mus. II, p.5 . . Sumatra. ruficaudis CAXD. Ann. Mus. Gênes, 1880, p. 197. ”

subopacus CAND. Tbid. . . . . . . »

erraticus Caxd. Bull.Soc.Ent.Belg.xvmi,p.127. Philippines. posticus CAND. Ibid. . . . . . . Mindanao.

3e Groupc.

Essèce polynésienne.

puberusus Montr. Ann. Fr., 1360, p. 259

(Adrastus) . . . . . . . . N.Calédonie.

SiLests.

CAND. Monogr. IV, p. 458.

Adrastus pars ERICHS.

Front non acuminé en avant, le reste comme chez les Glyphonyx. 
rutilipennis Illig. Mag. VI, p. 8 . . . Europe mér. occ. cordubensis Heyd. Deuts. Ent. Zeits. 1882. . Espagne. terminatus ERICHs. Zeitschr. III, p. 116. . Rég. méditerr. dimidiatipennis Reiore, Ann. Fr., 1856. . Rég. méd.or. concolor DesBr. Opusc. entom. I, 1875 . . . ” absimilis CAND. Monogr. IV, p.463 . . . Chine. modestus CAND. Élat. nouv. II, p. 52 . . . . ” mutabilis Bates, Proc. Zool. Soc., 1866 . . . Formose. musculus CAND. Mém. Soc. Liége, 2 sér. V. . Japon. sanguinicollis CAND. Monogr. IV, p. 461 . . Bengale. bengalensis CAND. Élat. nouv. IV, p. 56 . . . ” sericeus CAND. Ibid. II, p. 52. . . . . . . Sikkim. hilaris CAND. Monogr. IV, p. 461 . . . . . Ceylan. simulatus CAND. Ibid. . . . . . . . Madras. semirufus CAND. Ibid. . . . . . . . . Birmanie. semicastaneus CAND. Ann. Mus. Gênes, 1888, p. 688. . . . . . . . . . . . Birmanie. rufus CAND. Ibid. . . . . . . . . . . . » Gestroi Cand. Tbid. . . . . . . . . . ”

Ctemoplus.

CAND. Monogr. IV, p. 463.

Mésosternum à bords tranchants et horizontaux; tarses simples.

javanus CAND. loc. cit.

sanguinolentus CAND. Notes Leyd. Mus. II (Silesis) . Java. collaris CAND. Élat. nouv. IV, p. 57
Adrastus.

Eschs. Thon, Arch. II, I, p. 35.

Fossette mésosternale à bords déclives; hanches postérieures étroites; prothorax muni à la base de sillons plus ou moins longs; tarses sans lamelles. 
limbatus FABR. Mantiss., p. 235. . . . . . Europe. pusillus FABR. Syst. Eleuth. II, p. 246.

nitidulus MaRsh. Ent. brit.

axillaris ErICHS. Zeitschr. III, p. 122. . . . ”

turcicus Stient. Wien. Ent. Monats V, p. 219. Allanie.

pallens FABR. Ent. Syst. 1, 2, p. 132 . . . Europe.

จ. limbatus $\mathrm{P}_{\mathrm{AXK}}$. Fn. suec. III.

v. pusillus Herbst, Käf. X.

lacertosus Erichs. Zeitschr. ШU, p.126 . . . Europe mér. nanus Herbst, Käf. X, p. 96 . . . . . . . Europe.

I luteipennis Errchs. Zeitschr. III, p. 125 . . Autriche. humilis ERICHs. Ibid. . . . . . . . . Europe.

rudis Küst. Käf. Europ. 14, p. 33.

Miegi Graërrs, Mém. Map. Géol., 1858, p. 46 . Castille.

\section{TRIBU XXVI.}

\section{CAMPYLITES $\left({ }^{\prime}\right)$.}

Prosternum sans mentonnière; hanches intermédiaires contiguës; facies des Élatérides altéré, pour se rapprocher de celui des Cébrionides.

\section{Oestodes.}

LEC. Rev. Elat. in Trans. phil. Soc. X, p. 424.

Front rebordé; antennes dentées, ì partir du 30 article chez les mâles. Sutures prosternales courbes.

tenuicollis RAxD. Bost. Journ. II, p. 14 . . Etats-Unis sept. v. graciliformis RAND., loc, cit.

puncticollis Hors, Ent. Soc.Trans, 1874, p. $24 . \quad$ »

(1) Cette tribu devrait, logiquement, s'appeler Lepturö̈dites, le nom de Campylus étant tombé en synonỵmie. J'ai cru toutefois pouroir lui conserver le nom sous lequel elle a été créée par Lacordaire dans son Genera et consacrée dans d'autres ouvrages. 


\section{BLadus.}

LEC. Class. north Am. Col., 1861, I, p. 161.

Voisin du précédent, article 3 des antennes petit; sutures prosternales droites.

quadricollis SAX, Trans. Am. phil. Soc. VI, p. 186. . . . . . . . . . . Etats-Unis.

\section{Phanophorus.}

Sor. Gay, Hist. de Chil. Zool. II, p. 27.

Campyloxenus Farrur. Col. Chil., 1863.

De grosses vésicules phosphoriques sur le prothorax.

niger Sor., loc. cit. . . . . . . . . . . Chili. parallelus Soc. Ibid.

pyrothorax FAIRM., loc. cit.

\section{LEPTUROIDES.}

Herbst, Fuessly Arch. V, 1784, p. 103.

Campylus Fischer, Entomogr. Imperii russici II, p. 453.

Exophthalmus Latr. - Hammionus MegerLe.

Denticollis Pmu. et Miтт. ( $\left.{ }^{1}\right)$.

Front fortement caréné transversalement en avant; yeux petits, globuleux, saillants; antennes souvent pectinées chez les mâles; prothorax petit, inégal; élytres molles, larges; tournure de Lycide.

rubens Pri. et Mrtwensp. It. Poseg., p. 86 . . Europe or. denticollis FABR. Ent. Syst. IV.

pyrrhopterus Oz. Entom. II, p. 31.

miniatus CAND. Notes Leyd. Mus. VII, p. $121 . \quad J a p o n$.

(1) Suivant quelques entomologistes, ce nom, plus ancien d'un an que celui de Lepturoides, devrait prévaloir. Les partisans de la prescription trentenaire préconisent le nom de Campylus. J'adopte ici la dénomination de Herbst, sa construction se conformant davantage à ce qui est exigé pour les noms de genres. 
linearis Lrvwé, System. Nat. Ed. 10, p. 404.

Europe.

+ mesomelas Linné, Tbid.

cantharoides Ourv. Entom. II, p. 31.

dispar. PAYK, Faun. suec. ШI, p. 37.

var. livens, marginatus, marginellus $\mathrm{F} A \mathrm{BR}$.

bicolor $\mathrm{Pz}$. - lividus MULu. - limbatus ScHr.

Kiesenwetteri Schauf. Isis, 1862, p. 199 .

parallelicollis AđBÉ, Ann. Fr. 1850, p. 336.

Castille.

nigricollis Gebler, N. Mém. Mose. II, p. 14 . Sibérie or. incequalis CAND. Deuts. Entom.Zeitschr. 1879,

p. 282.

dilutiangulus Mотsсн. Schr. Reis. II, p. 112 .

Daurie. mongolicus Мотьсн. Tbid. . . . . . Mongolie. borealis PAXK, Faun. suec. II, p.6 . . . Anc. Cont. sept. cinctus CAND. Monogr. IV, p. 479 . . . . . Sibérie. Davidis FaIRMr. Ann. Fr., 1889, p. 34 . . . . Chine centr. variabilis Eschs. Thon, Arch. II, I, p. 33 . . Kamschatka.

จ. varians, Salllbergi Germ. Linn. Entom.I,p.153.

flavipes GERr. Linn. Entom. I, 1846, p. 155. productus RAND. Bost. Journ. II, p. 8 . . . Caroline. denticornis Krrsp, Fn. bor. Am. IV, p. 145 . Canada. flavinasus Melsh. Proc. Ac. Phil. II, p. 219. fulvus Mотsch. Bull. Mos., 1859, p. 386 . . . Californie.

CAMPYLOMORPHUS.

JACQ. Duv. Gener. Col. Europ. III, p. 130.

Caractères des Lepturoides; exceptionnellement une mentonnière au prosternum,

homalisinus Illig. Mag. VI, p. 14. . . . . Portugal. suturanigra (Athous) CBevr. Rev. Zool., p. 15.

Macromalooera.

Hope, Trans. Ent. Soc. Lond. I, p. 13.

Antennes extrêmement longues chez les mâles, très courtes chez les femelles. 
ceramboides Hope, loc. cit. . . . . Adelaïde, Swan Riv. cœnosa Hope, loc. cit. . . . . . . . . . »

Pleonomus.

MÉnÉtriḱs, Mém. Ac. Pétersb. VI, p. 48.

Ictis CAND. Mon. IV.

Antennes filiformes; angles postérieurs du prothorax spiniformes; corps svelte chez les mâles, épais chez les femelles.

tereticollis MÉnÉTR. loc. cit. . . . . . . Turcoménie. spinicollis FALDERMr. Tbid., 1835, p. 414 . . . Mongolie. sinensis CAND. Monogr. IV, p. 240 ( + Ictis). . Chine.

б acutidens FaIRM. Ann. Fr., 1878, p. 110.

strictus CAND. loc. cit., p. 498 . . . . . . Espagne.

\section{NOMOPLEUS.}

Cand. olim sub nomine Pleonomus.

Guerini CAND. Monogr. IV, p.500 . . . . Nilgherries. argentatus CAND. Ibid., p. 499 . . . . . . Madagascar. longicornis Boнемr. Ins. Caffr. 1, 2, p. 392 . . Cafrerie. misellus Bонем. Ibid. . . . . . . . . . . » Walbergi CAND. Élat. nouv. II, p. 54. . . - ” niger CAND. Tbid. . . . . . . . . ”

\section{CyLINDRODERUS.}

Eschs. Sпв. Rev. IV, 1836.

Cebrio GERM. Ins. Sp..n. 61.

Corps long, cylindrique; antennes longues, velues, linéaires; prothorax étroit, parallèle; hanches postérieures brusquement élargies en dedans.

femoratus Germ. Ins. Sp. n. p.61 . . . . . Brésil. indutus CAND. Monogr. IV, p. 504 . . . . . ” lineatus CAND. Ibid., p. 505 . . . . . . . ” stenoderus CAND. Ibid. . . . . . . . . . " 
relictus CAND. Ibid., p. 506.

Brésil.

vittatus CAND. Ibid.

Uruguay. mexicanus CAND. Ibid. . . . . . . . Mexique. chilensis CAND. Élat. nouv. II, p. 54 . . . . Chili.

Stichотомеs.

Cand. Monogr. IV, p. 507.

Antennes pectinées chez les mâles à partir du 30 article qui est en outre très long.

corrigiolatus CAND. loc. cit. . . . . . . Australie.

OcTINODES.

CAND. Monogr. IV, p. 487.

Antennes des mâles longuement flabellées à partir du 40 article, les appendices garnis de cils fins hérissés.

capillatus CAND. loc. cit. . . . . . . . . Vénézuéla. plumosus CAND. Élat. nouv. II, p. 53 . . . . Amazones. æquatorius CAND. Tbid. IV, p.57 . . . . Rịo Napo.

Plectrosternus.

Lacond. Génér. des Coléopt. IV, p. 227.

Oxysternus Latr. Ann. Fr. III, p: 164.

Antennes pectinées à partir du 40 article chez les mâles; prothorax court, présentant de longs sillons basilaires latéraux; élytres larges, amples, dilatées en arrière.

rufus (Latr.) Lac. Génér. IV, p. 228 . . . . Indes orient.

\section{HeMrops.}

(Eschs.) Lap. de Cast. Silb. Rev. IV, p. 15.

Antennes dentées dans les deux sexes; prothorax et élytres comme chez les Plectrosternus.

crassa Gxum. Schönh. Syn. App., p. 155. . Indes orient. nigripes, flava Cast. loc. cit. CAND. Mon.

chinensis, lutea Germ. Zeitschr. IV, p. 52.

plana CAST. Hist. Nat. I, p. 254. 
nigricollis CAND. Monogr. IV, p. 496 . . . . Bornéo. sinensis CAND. Élat. nouv. III, p. 106. . . . Chine. alternata FAIRM. Ann. Fr., 1878, p. 110 . . . ” " Semperi Cand. Bull. Soc. Belg. XXI. . . I. Philippines. acutangulata CAND. Élat. nouv. III, p. 105. Poulo-Pinang. longa CAND. Ibid., p. 106 . . . . . . . Sumatra.

\section{Adolesches.}

Cand. Élat. nouv. III, p. 107.

Représente les Hemiops en Amérique.

crinitus CAND. loc. cit. .

Uruguay.

\section{PARHEMTOPS.}

CAND. Élat. nouv. II, p. 53.

Dernier article des palpes cylindriques; antennes fortement dentées; voisin des Hemiops.

palliata CAND. loc. cit.

Siam.

angusta CAND. Notes Leyd. Mus. II, p. 5 .. . Sumatra. dubia Flevt. Ann. Fr., 1889 . . . . . . . Indo-Chine.

\section{HEMTOPSIDA.}

Mac Leay, Trans. N. S. W. II.

Mastersi M. L. loc. cit. . . . . . . . Queensland.

\section{Hemiopinus.}

FAIRM. Stettin. Zeitung., 1882, p. 385.

Hildebrandti FaIRM. loc. cit. . . . . . Madagascar. metallicus CAND. Élat. nouv. TV, p. 57

\section{Isosoma.}

Ménétr. Cat. rais. 1832, p. 160.

elateroides MÉń́tín. loc. cit.

Caucase. 


\section{TRIBU XXVII.}

\section{PLASTOCÉRITES.}

Facies de Cébrionides; antennes généralement pectinées chez los mâles; élytres très raccourcies et laissant les segments postérieurs en grande partie découverts chez les femelles; dernier article des palpes cylindrique; mandibules saillantes.

\section{Puastocertus.}

Leconte, Trans. Am. phil. Soc. X, p. 502.

Antennes de onze articles, pectinées dans les deux sexes où les élytres sont également semblables.

angulosus Gersr. Fn. Ins. Europ. 23, p. 5 . . Asie mineure Schaumi LEc. loc. cit. . . . . . . . . Californie.

v. frater LEc. Proced. Acad., 1859, p. 73.

v. macer HonN, Trans. Am. Entom. Soc., 1884.

Aplastus.

Led. Proc. Ac. Philad., 1859, p. 73.

Anamesus Lec. Proc. Ac. Phil., 1866.

Antennes des mâles fortement dentées à partir du 40 article; élytres des femelles très courtes.

angusticollis HoRN, Tr. Am. Ent. Soc., 1874, p. 25 . . . . . . . . . . . Californie. tenuiformis Hons, Ibid. . . . . . . . . » corymbitoides HonN, Ibid. . . . . . ” speratus Lec. Proc. Acad. Phil., 1859, p. 73 . ” optatus LEc. Tbid., 1861, p. 349 . . . . . . ”

+ Anamesus convexicollis LEc. Ibid., 1866, p. 393. molestus Hors, loc. cit., p. 27 . . . . . . ” 


\section{Euthisantus.}

LEC. Trans. Am. phil. Soc. X, p. 502.

Antennes de 12 articles, fortement pectinées chez les mâles à partir du $4 \theta$ article ; élytres très raccourcies chez les femelles.

lautus LEC. loc. cit. . . . . . . . . . Californie. pretiosus LEc. Ibid. . . . . . . . . . »

\section{APHRICUS.}

LEc. Trans. Am. phil. Soc. X, p. 501.

Fàcies de Cardiophorus; antennes de 11 articles simples chez les mâles, seul sexe connu.

californicus LEC. loc. cit. . . . . . . Californie.

\section{ENISONYX.}

HoRN, Tr. Am. entom. Soc., 1884.

Facies de Cardiophorus, y compris la forme de l'écusson, le reste comme chez les Aphricus.

pullatus Horn, Trans. Ent. am. Soc., 1884, p. 52. Californie. gracilis Hons, Ibid. 



\section{ADDITIONS ET CORRECTIONS.}

p. 23, ligne 11 : Henzi, lisez Herzi.

p. 88, 1. 14, aj. en synon. Ampedus GerM.

p. 89, l. 1 : Elater sanginolentus Schr. aj. en syn. E. lythroptrus, GERM.

p. 124, 1. 24, aj. G. Russowi Herd. Deutsch. entom. Zeits., 1889, p. 328 . . . . . . Turkestan.

p. 130, 1. 18, aj. G. cruciatus CAND. Élat. nouv. IV, p. 41. . . . . . . . . . . Madagascar.

p. 102 : Oxysthetus, lisez Oxystethus.

p. 162, 1. 3, supprimez NiGer SoL. reporté p. 211, sous le nom générique de Phanophorus.

p. 188 : Les trois espèces du G. Mecastrus ont été, par erreur, imprimées en caractères italiques. 



\section{AUTEURS ET OUVRAGES CTTÉS.}

AUBÉ (Charles). Description de quelques Coléoptères appartenant à l'Europe et à l'Algérie, in Ann. Soc. entom. Fr. 1850.

BACH (Michael). Käferfauna für Nord-und Mitteldeutschland 18491860.

BAILLON (E.). Verz. Kreise v. Kuldsha ges. Käf. in Bulletin de Moscou, 1878 (Cor. atratus).

Bates (Henri). Insectes de l'île Formose in Proceedings Zool. Soc. 1866.

BAUDI (Flaminio). Berliner Zeitschrift, 1859.

- Ibid., 1871.

_ in de Marseul " L'Abeille " 1871.

BERToloni (Giuseppe). Illustratio rerum naturalium Mozambici. Dissertatio III, 1849.

- Memor. Acad. Bol. Sér. II, IV.

BJERKANDER (Clas). Beskrifning pà et högst etc. (Elater segetis) in Vetensk Acad. nya Handl., 1779.

Blanchard (Emile). Insectes du "Voyage dans l'Amér. mér. d'Alc. d'Orbigny , 1837-1843.

- Voy. au Pôle Sud. Insectes recueillis par MM. Hombron et Jaquinot, 1853.

BLANCHARD (Frédér.). Cardiophorus américains in Trans. Amér. entom. Soc., 1889.

BLAND. Proc. entom. Soc. Phil., 1864.

BOHEMAN (Carl. H.). Insecta Caffraria a Wahlberg collecta, T. I, fasc. II, 1851.

- Entom. und. Resa I, Södra Sver. in Vet. Akad. Handl., 1852.

- Kongl. Sv. Freg. " Eugenies „ Resa, 1858. 
Borsduval (Dr Alph.). Fn. entom. de l'Océan Pacifique in Voy. de l'Astrolabe, 1832-1835.

- (et Lacordaire). Fn. entom. des environs de Paris, I, 1835.

Brisout de BArveville (L.). Nouv. coléopt. français in Ann. Soc. entom. Fr., 1861.

- Ibid. 1866 (espèces d'Espagne).

Broun (Capne Thomas). New Zealand Journ. Sc. 1880.

- Ibid, 1882.

- Man. new. Zeal. Coleopt., 1885.

BRuldé (Auguste). La partie des Insectes dans l'Expédition de Morée, 1832.

Burmeister (Herman). Col. de la Rép. Argentine in Stett. entom. Zeit., 1875.

Buysson (Henri du). Annales de la Soc. entomol. de France. Bullet. 1889 .

- Ibid., 1890.

CandĖze (Ernest). Monographie des Elatérides in Mémoires de la Société royale des Sciences de Liége; XII, 1857 (T. I); XIV, 1859 (T. II); XV, 1860 (T. III) ; XVII, 1863 (T. IV). Il y est décrit 1988 espèces, dont plus de la moitié nouvelles.

- Elatérides nouveaux, fasc. I, in Mémoires in-80 de l'Académie des Sciences de Bruxelles, XVII, 1865. Description sommaire de 165 espèces.

- Elatérides du Japon in Mémoires de la Société Royale des Sciences de Liége, $2{ }^{\circ}$ sér. T. V, 1873.58 espèces, 44 nouvelles.

- Révision de la Monographie des Elatérides, T. I (seul paru) in Mémoires de la Soc. Roy. de Liége, $2^{e}$ sér. IV, 1874. Ne comprend que les premières tribus et s'arrête aux Tetralobus exclusivement. 158 espèces y sont décrites pour la première fois.

- Elatérides nouveaux. Fascicule II, in Bulletin de la Société entomol. de Belg., 1881. 198 espèces. 
CANDĖzE (Ernest). Relevé des Elatérides recueillis dans les îles Malaises, etc., par MM. Doria, Beccari et d'Albertis in Annali del Museo civico di Genova XII, mars 1878.

- Addition au Mémoire précédent Ibid XV, 1880. Ces deux mémoires renferment 149 espèces, dont plus de la moitié nouvelles.

- Descriptions of the new Elateridæ collected during the recent scientific Sumatra Expedition in Notes from the Leyden Museum II, 1879.

- Un genre et deux espèces d'Elatérides. Ibid. Même année.

- Une espèce africaine d'Elatéride. Ibid., 1882.

- Description de trois espèces nouvelles d'Elatérides de l'Archipel Indo-Néerlandais. Ibid. V, 1883.

- Deux Élatérides nouveaux des îles de la Sonde. Ibid. $\nabla, 1883$.

- Elatérides récoltés à Serdang, par le Dr Hagen. Ibid. V, 1883.

- Deux espèces nouvelles d'Elatérides. Ibid. XII, 1884. Ces différents mémoires font connaître 30 espèces.

- Elatérides nouveaux. Fascicule III in Mémoires de la Soc. des Sciences de Liége, $2{ }^{\ominus}$ série, T. IX; 242 espèces y sont décrites.

- Note sur un Elatéride de Madagascar du groupe des Allotriites in Cistula Entomologica, Aug. 1879,

- Elatérides de l'Amur in Deutsch. Entom. Zeitschr., 1879.

- Notes sur les Elatérides du genre Chalcolepidius in Bulletin de la Soc. entomologique de Belgique, arril 1886. (58 espèces, dont 7 nouvelles.)

- Elatérides nouveaux. Fascicule IV in Annales de la Soc. entom. de Belgique, XXXIII, 1889. 200 espèces.

- Elatérides recueillis en Birmanie et au Ténassérim, par M. L. Fea, in Annali del Museo civico di Genova, sér. 2, vol. VI. Décembre 1888. 90 espèces, dont 37 nouvelles.

- Bull. Soc. Zool. Fr., 1889.

- Note sur les Elatérides du Chota-Nagpore in Bulletin de la Soc. entom. de Belgique. Sept.1890. 52 espèces, dont 16 nouvelles. 
CANdĖze (Ernest). Note sur un Chalcolepidius nouveau in Bull. Soc. entom. Belg. Nov. 1890.

Casey (Thos. L.). Contribution to the descriptive and systematic Coleopterology of N. Amér., II, 1885.

Castelyad (Laporte de). Revue entomologiqne de Silberman, 1831.

- Tbid. 1836.

- Histoire naturelle des Insectes; Coléoptères I, 1810 (162 espèces, dont 35 données comme nouvelles).

Charpentier (Toussaint de). Horæ entomologicæ. Breslau, 1825.

Chevrolat (Auguste). Coléoptères du Mexique, centur. II, 1835. (plusieurs Chalcolepidius, etc.).

- Revue Zoologique, 1841 (Camps rutilans).

Ibid. 1852 (Cor. Racinei).

- Magasin de Zoologie, 1843.

- Annales de la Soc. entom. de France, 1867 (Coléopt. de Cuba).

Costa (O. Gabriel). Voyage en Calabre, 1881.

Cowper (Willam). Canad. Natur.; 1865 (Athous affinis).

Crotch (G. R.). Proceed. Zool. Soc. London 1867 (Elastrus dolosus).

- Check list of the Coleopt. of America, North of Mexico, 1880.

CuRTIS (John). British Entomology; Ins. found in Gr. Brit. and Irel. 1823-1840.

DEJEAN (Pierre Fr. Cte). Catalogue des Coléoptères de sa collection. L'édition de 1833 énumère 20909 espèces, au nombre desquels 600 Elatérides dont beaucoup d'inédits. C'était l'une des collections les plus riches de l'époque.

Desbrochers Des Loges (Jules). Espèces nouvelles de Coléoptères; in Abeille VII, 1870.

- Coléoptères d'Eur. et confins de la Méditerranée, in Mitt. Schweis. Entom. Geselsch. 1870-71.

Desvignes (Thomas). New British Elater; in Entomologist, 1842.

DEYROLLE (Achille), in Maillard, insectes de la Réunion.

DOHRN (Ch. Aug.). Stettiner Entomol. Zeitschr., 1881. 
Drapiez (A.). Descriptions d'insectes, nouveaux; in Ann. génér. des Sc. phys., Brux. 1819.

DRURY (Drew). Illustrat. of natur. History, 1770-1782.

Dufour (Léon). Excurs, entomol. dans la Vallée d'Ossau; in Bull. Soc. Sciences de Pau, 1843.

Dumont (Gustave). Description d'une nouv. esp. de Dichronychus et d'Adiaphorus; in Bull. Soc. entom. Belg., 1890.

ERICHSON (W.F.). Ueber Elateriden ohne Bruststachel (Cardiophorus); in Germar, Zeitschr. f. d. Entomol. II, 2, 1840. (109 espèces sont décrites, beaucoup de nouvelles. L'auteur comprend dans ce genre toute la tribu actuelle des Cardiophorites.)

- Die Arten der Gat. Chalcolepidius; in Germar Zeitschr.III, 1841. L'auteur y parle de 18 espèces, plusieurs nouvelles.

- Ueber die Elateren mit Kammförmig gezähnten Krallen. 40 Melanotus (1 Perothops), 15 Adrastus (Silesis et Glyphonyx compris).

- Beitrag zur Insekten fauna V. Vandiemensland; in Arch. fur naturgeschichte VIII, 1842. (16 espèces et deux genres nouveaux, Crepidomenus et Atelopus.)

- Meyens Reise; in Acta Acad. Leop. Carol., 1834.

- Conspectus Ins. Coleopt. quæ in Republica peruaia observata sunt; in Wiegm. Arch., 1847.

- Die ins.; in Schomburgks Reise in Guyana, 1848.

- Insecten Fauna von Angola; in Wiegm. Arch., 1843.

EsCHS CHOLTZ (Jos. Fried.). Entomographien; in Dorpat, naturw. Abhandl. 1823.

- Eintheilung der Elateriden in Gattungen; in Thon Archives, 1829. Important travail. C'est le premier ouvrage d'ensemble où le genre Elater est transformé en famille et divisé en 28 genres de nouvelle formation.

- Die Quatember, Zeitschr. f. Naturwissenschaft, 1829. (Elat. ochropterus.)

- Tableau des genres d'Elatérides; in SrLb. Revue entomol. 1836. 
FABRICIUS (Joh. Christ.). Systema Entomologiæ, 1775.

- Species insectorum exhib. diff. spec, 1781.

- Mantissa Insectorum, 1787.

- Entomologia systematica, 1792-1794.

- Systema Eleutheratorum, 1801. Il y est décrit, sous le nom d'Elater, 137 espéces, dont beaucoup d'exotiques, à peine signalées alors.

- Index alphabeticus; in System. Rhyngot, etc., 1803.

Fairmaire (Léon). Coléoptères de la Polynésie; in Rev. et Magas. de Zool., 1819.

- Annales de la Soc. entom. de France, 1852.

- Ibid. 1866, Ibid. 1871, Ibid. 1878, Ibid. 1881, Ibid. 1881. Ibid. 1887.

- Journal " le Naturaliste " 1880 (Elat. de la Réunion) 1883 (N. Bretagne) 1885 (Somalis) 1885 (Terre de Feu).

- Revue Zoologique, 1860 (Col. du Chili).

- Stettiner Entom. Zeitschr. 1883.

- Ann. Soc. entom. Belg., 1883 (Niles Hébrides).

FALDERMANx (Franz). Fauna entomologica transcaucasica 1835-1838; in Bulletin de Moscou. N. Mém.

- Col. ab ill. Bungio in China boreali, Mongolia, etc. Collect. 1835.

- N. Mém. des Nat. de Moscou. Coleopt. persico-armeniaca. Addimenta, 1835.

FAuST (J.). Horæ Societ. entom. Rossicæ 1877. (Ath. Raddei, Astrabadensis.)

Fauvet (A.). Coléoptères de la Nlle Calédonie; in Bull. Soc. Linn. Norm. VII, 1862.

- Cat. des ins. rec. à la Guyane française, par M. E. Déplanche; in Bull. Soc. Linn. norm. V, 1861.

Fischer voN WALDHEN (Gotthelf). Catalogus coleopt. in Sibiria orientali a Gregorio Karelin collect. 1842.

- Entomographia Imperii russici, 1820-1822. 
Fideutiaux (Edm.). Annales de la Soc. entomol. de France, 1889. Elatér. de l'Indo-Chine.

Fourcrox (Ant.-François). Entomologia parisiensis, 1785.

FrIvaldski (Emerich). A Magyar tudos Tàrsasàg, etc. in Ann. der Ungr. Akadem. 1835. (Coléopt. des Balkans).

- Akirályi magyar, etc.; in Annal. der. nat. Ges. V. Ungarn, 1835.

Froelich (Jos. Aloys). Neue Gattungen und Arten von Käfern; in Naturforscher. 1799.

FÜESSLY (Johan. Gaspar). Verzeichniss Schweiz. Ins., 1775

- Archiv der insekt., 1781-1786. Herbst a inséré dans cette publication la description de beaucoup d'Elater.

GEBLER (Fried. Aug. von), Ledebours Reise Bemerkung. über die insect. Sibiriens, 1830.

- Sibirischen coleopteren; in Bull. Acad. St-Pétersb., 1841.

- Verzeichniss der in Koliwano-Woskresenskichem, etc.; in Bull. Moscou, 1847.

GEER (Carl de). Mémoires pour servir à l'histoire des insectes, 17521778.

GEMMTNGER (Max.). Voy. HAROLD.

GÉNÉ (Carlo Giuseppe). de Quibusd. Insectis Sardiniæ novis aut minus cognitis; in Mém. Academ. Torin. 1836.

GERMAR (Ernest Fried ). Reise nach Dalmatien, etc. II, 1817.

- Fauna insectorum Europæ, 1817.

- Insectorum Sp. nov., 1824.

- Ahandl. Berl. Akad., 1832.

- Elatérides à tarses lamellés; in Zeitschrift für die Entomologie 1, 2, p. 193 à 236, 1839, établit les G. Eucamptus, Semiotus, Tetralobus, Hemicrepidius, Dicrepidius, Dipropus, Heteropus, Atractodes, Hypodesis, Monocrepidius, Pomachilius, Dima, Synaptus.

- Bemerkungen über Elateriden; in Zeitschr, f. d. Entom. II, 2, p. 241-278, 1840. Il traite des genres Physorhinus, Tylotarsus Dicronychus, Agrypnus (pars), Adelocera, Lacon, Iphis Ctenicera, Hemirhipus. 
- Beitrage zu einer Monogr. d. G. Pyrophorus Ill.; in Zeitschr. f. d. Entom. III, 1841.69 espèces y sont décrites - plusieurs où les sexes sont sous des noms différents.

- Bemerkungen über Elateriden; in Zeitschr. IV, 1843.

Crepidomenus, Ludius, Biliophorus, Tomicephalus, Hemiops, Corymbites, Diacanthus, Pristilophus, Cardiorhinus.

- Die Arten des gat. Campsosternus Latr. Ibid. IV, 1843, p. 99. 18 espèces.

- Bemerkungen über Elateriden; in Zeitschr. V, 1844. Monogr. des genres Cryptohypnus, Ampedus, Ischnodes, Aphanobius.

- Beitrage z. insekten Fauna von Adelaide; in Linnea entomologica, t. III, 1848. - Id. t. I, 1846 (Campylus).

GERStAECKER (Carl). Beitrag zur Insekten-Fauna ron Zanzibar; in Bull. Berl. Akad, juin 1871. Courtes diagnoses de plusieurs espèces d'Elatérides.

- Iarhb. Hamburg. Anst. 1883 (Alaus polyzomus).

Gestro (Dr Raphaelo). Descript. de Alcune Nuov. Sp.; in Ann. museo civic. di Genova, 1875.

GoRY (Hypolithe). Description de l'Elater Goryi; in Magasin de Zool., 1832.

- Description d'un Tetralobus nouveau; in Ann. Soc. Entom. Fr. 1832.

- I'rois Coléoptères nouveaux. Tbid. 1832.

- Tetralobus et Scarabeus nouveaux. Ibid. 1836.

Gozis (M. des). Recherche de l'espèce typique, 1886.

Graells (Mariano de la Paz). Zool. espagnol.; in Memorias de la comm. del Mapa Geolog., 1858.

GredLer (M. Vinc.). Coléoptères du Tyrol (Käf. Tyr.) 1833.

GUÉrin-NÉneVILLE (F. S.). Toyage autour du Monde de la Corvette "La Coquille" sous les ordres de Duperrey, 1829-1832.

- Insectes du voyage de "La Favorite „; in Magas. de Zool., 1838.

- Voyage de G. Osculati au Rio Napo; in Verh. Wien Zool. Bot. Ver. 1855. 
- Voyage de Lefebure en Abyssinie; in Revue Zoologique, 1847.

- De nombreux articles dans les Revues: 10 Magasin de Zoologie, 1831-1845; 20 Revue zoologique, 1838-1848; 3o Revue et Magasin de Zoologie, 1849 et suiv.

Guillebead. Voy. Mulsant.

GyLlenhali (Leonhard). Insecta suecica. Dans le vol. 1, il est décrit 52 Elaters, dont plusieurs nouveaux.

- in Schönherr Synon. Insector. Appendix 1817.

Haldeman (S. S.). Descript. of insects, etc.; in Proceed. Acad. Nat. Sc. Philad. 1844.

HAMPE (Clem.). Beschreib, einig. n. Käferart. (Ath. foveolatus); in Stettin. Zeitschr. 1850.

- Athous proximus, etc.; in Wien Entom. Monatsber. 1864.

- El. cuneiformis, etc.; in Berlin. Entom. Zeitschr., 1866.

HAROLD (Edgar von). Catalogus coleopterorum, T. V (Elatérides) 1869 (avec Gemminger).

- Deux Elat. du Congo; in Munch. Entom. Ver. 1878.

HARRIS (Taddeus Will.). Trans. Hartfort Nat. History, 1836 ( $E l$. militaris).

Hentz (Nicol. Marcel). Descript. of some n. Sp. of N. Amer. Ins. Col.; in Journ. Acad. Sc. Philad. 1827. (Cor. vernalis.)

Herbst (Joh. Friedl. Will.). In Fuessly's Archiv., 1791.

- Natursystem. all. Bekanten in und ausl. insekten Käfer 1-10, 1785-1806.

HEYDEN (Luc. von). Entom. Reise in Span. Berl. ent. Zeitschr. 1870 .

- Senck Nat. Ges. Francf. 1877.

- Deutsches Entom. Zeitschr., 1880 à 1886. Différents articles principalement sur des Elatérides d'Asie.

- Verh. Verein Brünn. T. XVI.

HOPE (Fr. Will.). In Gray, Zool. Miscell., 1831.

- in Griffith, Animal Kingsd., 1832.

- Monogr. of the col. fam. Phyllophorida; in Proceed. Zool. Soc. Lond. (G. Leptophyllus, Pectocera, Tetralobus, Piezophyllus, etc.) 
- Monogr. of G. Campsostermes; in Ann, and Magaz. of Nat. Hist. 1842.

- On some insects of Western Africa. Ibid. 1842.

- 22 esp. africaines; in Ann. Nat. Histor. 1843. Non reconnaissables.

- Trans. Entom. Soc. Lond. 1815. Même remarque.

HonN (Dr George). Transact. Amer. Entomolog. Society. Presque tous les vol. de cette Revue renferment des revisions de genres et des descriptions d'espèces nouvelles des EtatsUnis. Je citerai notamment :

Ann. 1871. Revision des Adelocera et espèces nouvelles.

Ann. 1880. Sur les Asaphes de l'Amér. boréale.

Ann. 1881. Sur les Aphricus, Aplastus, Plastocerus, Euthisanius, avec figures.

Ann. 1884, Monogr. des genres (pour les Etats-Unis). Horistonotus, Esthesopus, Aptopus, Ludius, Anchastus, etc., etc.

ILliger (Joh. Carl. Wil.). Tierz. new insecten in Wiedem.Arch. 1800 (Elater (Ctenicera) nobilis).

- Magazin fur Insektenkunde, 1802-1807.

- Magazin Gesellsch. Nat.Freund.1807.Monographie der Elateren mit leuchtenden Fleck. auf den Halsschilde. 16 espèces qui prendront, plus tard, rang dans le G. Pyrophorus. Première monographie d'un groupe d'Elatérides.

- Fauna Etrusca, 1807.

JANson (Edw.). Cistula entomologica 1882, vol. III. (Ch. Bukleyi) Sem. carus, etc.).

JAQUelix-Duval (Camille). Coléoptères de Cuba; in Ramon de la Sagra. Hist. de Cuba, 1857.

- Genera des Coléoptères d'Europe, 1851-1868. Elat. en 1863. G. Campylomorphus.

KARSCH (D F.). Sitz. B. Natur. Freund. 1881. (Cten. controversa.)

- Deutsch. entomol. Zeitschr., 1881.

KIESENWETtER (Ernest Helmuth von). Naturgeschichte der Insecten Deutschl. IV, 1857-1861. 
- Beitrage z. Käferf. Griechenland; in Berlin. Entomolog. Zeits. 1859.

- Schaum catalog. 1862 (Pittonotus).

- Verh. Verein. Brun. XVI.

KirBY (William). Fauna boreali americana; Ins. du Voy. de Franklin 1837. (Plusieurs Elatérides du Canada et régions voisines.)

KIRSCH (Theodor). Plusieurs mémoires in Berlin. Entom. Zeitschr. notamment en 1866, Beitr. z. Kaferfauna von Bogota. Ibid. en 1870 , en 1873, en 1875, et en 1883.

- Mitt. Mus. Dresde 1875.

KudG (Joh. Christ. Fried.). Insectes de Madagascar; in Abhandl. d. Konigl. Akad. d. W. z. Berlin, 1832-1833.

- Verzeichniss von Thieren, etc. Reise von Erman, 1835.

- Peters Reise nach Mozambique, 1862. (Renferme la description de plusieurs Elatérides.)

KOLBE (H. J.). Arch. f. Nat. 1887-1888.

KoLENati (Friedr.). Meletemata entomologica, 1845 et suiv.

KolLaR (Vincent). In Hugel, Reise d. Kashmir. 1842.

KöNIG. In Horæ entomol. Rossicæ 1888. (Insectes de Corée.)

- Ibid. 1889. (Asie centrale.)

KraAtz (Gust.). Deutsch. entom. Zeitschr. 1879. (Elat. de l'Amur.)

- Ibid. 1883. (Adel. tristis, incompta, etc.)

KRINICKY (Johann). Enumeratio coleopterorum Rossiæ meridionalis; in Bull. Moscou, 1832. (Card. obesus, etc.)

KUSCHAKEWITSCH (J.). Eine neue Elateride (Corymbites Baeri); in Hor. Soc. Rossicæ, 1861.

KÜSTER (H. C.). Die Käfer Europas, 1844-1853 (beaucoup de fausses espèces).

LACORDATRE (Théodore) (avec Boisduval). Faune entomologique des environs de Paris, I, 1835. Il y est décrit quelques Elatérides nouveaux. Le 1er vol, seul paru, est tout entier de la main de Lacordaire. 
- Genera des Coléoptères, T. IV, 1857. Dans lequel l'auteur établit un nouvel arrangement des Elatérides et crée plusieurs genres.

LATreille (P. A.). Histoire naturelle des crustacés et des insectes, 1802-1805. Ouvrage fondamental.

- Les insectes du voyage de Humboldt et Bompland, 1811.

- Les insectes d'Afrique, recueillis par Caillaud. Voy. à Méroé, 1823.

- Familles naturelles du Règne animal, etc. Insectes, 1825.

- Distribution méthodique des Serricornes; in Annales Fr. 1834 (ouvrage posthume).

Le Conte (Dr John). American philosophical transactions. Vol. X, Oct. 1853. Renferme une Monographie des Elatérides des Etats-Unis, avec classification et création de nombreux genres. Sans les Eucnémides, qui y sont compris, le Mémoire renferme la description de 273 espèces, pour la plus grande partie nouvelles.

- Rep. Explor. and Surveys 1857. Limonius ornatulus, Athous scissus, Corymbites Suckleyi.

- Proceed.Acad. Nat. Sc. Philad. 1859. (Aplastus speratus, Plastocerus frater, etc., p. 69-90.)

- Ibid. 1854. (Chalc. smaragdinus, Webbi, etc.)

- Ibid. p. 281-292. (Adel. rorulenta, etc.)

- Ibid. 1866, p. 361-394. (Ad. maculata, pyrsolepis, Meg.angularis, Anchastus bicolor, etc.)

- Ibid. 1861. (Chalcolepidius rubripennis, etc.)

- Smithsonian Misc. Coll. 1863-66. (Alaus melanops, Horist. densus, simplex, Crypt. grandicollis, planatus, Mon. athoides, etc.)

- Ann. Mag. Nat. history, 1869. (Corymbites fraternus, etc.)

- Proceed. Acad. Phil., 1866. (Crypt. gentilis, Ath limbatus, Anch. bicolor, Melanotus opacipennis, etc., etc.)

- Trans. Am. ph. Soc. 1874. Scaptolenus estriatus. 
- Proc. Ac. Nat. Sc. Phil., 1860. Asaphes carbonatus, etc.

- Annual rep. Chief Engineers, 1876. Asaphes soccifer, etc.

- Bull. U. S. Geol. and Geogr. Survey, 1878. Corymb, planulus.

- Proceed. Am. ph. Soc. 1878. Anchastus asper, Athous debilis Isch. fuscus, etc.

- Ibid. Col. of Florida. Anchast. longulus, fuscus, 1878.

- Trans. Am. ent. Soc. XII, 1884. Etudes sur les genres et espèces de : Drasterius, Megapenthes, Elater, Agriotes et Dolopius des Etats-Unis.

LE GUILLou (Elie J. Fr.). Coléoptères recueillis dans le voyage de l'Astrolabe et de la Zélée; in Revue Zool., 1844.

LESKE (Gottfr. Nathan). Reise durch Sachsen, 1785.

LEWIS (George). Cat. des Coléoptères du Japon, 1877.

- Diagnoses d'Elatérides du Japon; in Entom. Monthley Mag. Décembre 1879.

LINNÉE (Carl.). Mus. Ludovicæ Ulricæ Reginæ, etc., 1764.

- Systema Naturæ. Ouvrage fondamental. On s'arrête généralement à l'Ed. XII, qui est de 1767 (l'Ed. XIII (Gmelin) est de 1788). Linné est le créateur du genre Elater, devenu depuis la Famille des Elatérides. Il signale une trentaine d'espèces. LUCAS (Hippolyte). Hist. nat. des animaux articulés de l'Algérie, 1879.

- Animaux rares, etc. rec. par Castelnau, dans la trav. de l'Amér. du Sud, 1857.

MAC LeAY (William). Notes on a collect. of ins. from Gayndah, etc.; in Trans. Entom. Soc. of New S. Wales. Vol. II, 1873. Description d'une trentaine d'Elatérides difficilement reconnaissables, l'auteur s'étant abstenu de comparaison avec les espèces voisines déjà connues et déjà fort nombreuses, par ex. des Lacon et des Monocrepidius d'Australie.

MAERKel (Joh. Christ. Fried.). Stettin. Entom. Zeitschr. 1847.

MAEkLIN (Fréd.). Ofvers Finsk. vet. Soc. XIX, 1877.

ManNerheim (Carl. G. von). Monogr. du G. Eucnemis. Il y est décrit l'Eucn. (Olophous) gigas. 
- Nov. Coleopt. species Imperii Rossici, otc.; in Hummel Essais, 1825.

- Bulletin de Moscou. Nombre de volumes de cette Revue renferment des articles de Mannerheim sur des Elatérides de Californie, de l'ancienne Amérique russe, etc. qui sont cités lorsqu'il y a lieu.

MARSHAM (Thomas). Entomologia britannica, 1802.

Marseul (S. A. de). Le journal l' "Abeille „. Elatérides dans les vol. V et VI (1868-1869).

MEgerle (Jos. Carl.). Catalogus insect. q. Viennæ Austriæ describ. 1801.

Melshenmer (Fried.). Descript. of n. sp. Coleopt. of the United States; in Proc. Acad. Philad. 1846.

MÉnÉtriés (E.). Catalogue raisonné des objets rec. dans un voy. au Caucase, 1832.

- Cat. d'ins. rec. entre Constantinople et les Balkans; in Mém. Acad. Pétersb. 1838.

- Catalog. des ins. rec. par Lehman; in Mém. Acad. Pétersb. 1848.

Muluer (L.). Wien Entom. Monatsb. 1862.

- Deutsch. Entom. Zeitschr. 1881. (Agr. cingulatus, nitidicollis, etc.)

Montrouzier (P.). Essai sur la Faune de l'Hle Woodlark; in Ann. Soc. d'Agric. de Lyon, 1857.

- Faune de la Nlle Calédonie; in Ann. Soc. Entom. Fr. 1860.

Motschulski (Victor de). Descriptions d'espèces d'abord sous le nom de "Victor ", puis sous son nom, dans un grand nombre de volumes du Bulletin de Moscou.

- Id. dans le Bull. de l'Acad. de St-Pétersb.

- Etudes entomologiques. Différents nos de cette Revue, commencée en 1853, sont consacrés à des descriptions d'espèces nouvelles, dont nombre d'Elatérides. Beaucoup de noms appliqués à ces espèces par l'auteur doivent (lorsqu'elles sont reconnaissables, ce qui n'est pas toujours le cas), tomber en 
synonymie, Motschoulski ne s'étant pas assez préoccupé des travaux parus avant lui.

- Coléoptères de la Sibérie orient. du voyage de Schrenk, etc. 1860.

Plusieurs Elatérides nouveaux.

MÜLLER (Otto Fried.). Zoologiæ danicæ prodromus, 1776.

MÜLLER (Philippe). Neue insecten Beschreib.; in Germ. Magaz. d. Entom. IV, 1821.

MULSANT (Etienne). Opuscules entomologiques, 1852-1860.

- Annales de la Soc. Linn. de Lyon, à partir de 1838. Souvent arec la collaboration de MM. Guillebeau ou Rey, de nombreux Mémoires contenant la description d'espèces nouvelles, principalement de l'Europe centrale et méridionale.

Murray (André). Ann. and Magaz. of nat. History, 1872.

NewMan (Edward). Ins. Coll. by Gibbon; in Trans. Ent. Soc. Lond. n. sér. IV, 1857. (Alaus Gibboni.)

OLIVIER (Ant. Guill.). Entomologie, ou Hist. nat. des Insectes, etc. 1789-1808.

- Journ. d'Hist. nat., 1792.

Palisot DE Beauvois (Ambr.). Insectes rec. en Afrique et en Amérique, etc. $1805-1821$.

PASCOE (Francis). Ann. and Magaz. of nat. Hist. 1876.

PAYKUL (Gustaf).. Insecta suecica (Coleoptera) 1798-1800.

Panzer (George Wolf. Fr.). Fauna insectorum Germanicæ initia, 1793-1809. Beaucoup d'espèces y sont décrites et accompagnées de bonnes figures.

- Deutsch.insekten Fauna, oder Entomol. Taschenbuch, etc. 1795. PELERIN (J.). Sur l'Elater punctolineatus; in Vigors Zool. Journal, 1829. Perez ArCaS (Laureano). Ann. Soc. esp. Histor. nat. 1872. (Dima Assoi.)

- Ibid. 1874 (Mel. Hidalgoi, etc.).

PERrIs (Edouard). Description de quelques esp. nouv. de Coléopt. (d'Espagne); in Annales Soc. entom. de France, 1864. 
PERroud (B. Philibert). Ann. Soc. Lin. Lyon 1864 (El. Guillebaui). PERTy (Maximil.). Delectus animalium articul, quæ colliger. Spix et Martius 1830-1834.

Phimrppr (F. H.E.). Species quædam novæ Coleopt. Chilens.; in Annal. Universit. Chile 1861.

- Stettiner entom. Zeitschr. 1873. (Cryptot. ater.)

PILLER (Mathias). Iter per Poseganam, Sclavoniæ provinciam, etc. (Avec Mitterspacher) in Scriba journ. 1783. (Dent. rubens.)

PODA (P. Nicolaus). Insecta Musæi græcensis, etc. 1761.

QUEDENFELd (M.). Deutsch. entom. Zeitschr. 1886. (Plusieurs Elatérides du Congo.)

RAGUSA (Emilio). Naturalista siciliano 1881. (Agr. hymerensis.)

RANDALI (John. W.). New species coleopt.; in Boston natural History II, 1838. (El. semicinctus.)

RAZUMOVSKY (Graf Geory). Histoire naturelle du Jorat et de ses environs, etc. 1789.

REDTENBACHER (Ludwig). Fauna austriaca, 1849.

- Reise Novarra II. (Acroniopus grandis.)

REDTENBACHeR (William). Qædam gener, et species Coleopt. Archid. Austriæ Dissertatio, etc. 1842. (Porthm. fulvus, etc.)

ReICHE (Louis). Coléopt. du voy. de Ferret et Galinier en Abyssinie, 1847.

- Col. rec. par M. F. de Saulcy dans son voyage en Orient (Agr. judaicus, etc.); in Ann. Soc. entom. Fr. 1856.

- Coléopt. N. rec. en Corse par M. Bellier de la Chavignerie. Ibid. 1861.

- Tbid 1864. (Ath. Peragalloi.)

- Catal. des Coléopt. de France de Grenier (3 Athous) 1863.

- Note sur quelques espèces du G. Athous; in Ann. Soc. entom. Fr. 1869.

Reitter (Edm.). Wien Entom. Zool. 1881.

- Deutsch. Entom. Zeitschr. 1887. 
- Entomol. Nachrichten, 1890, no 16. Plusieurs nouveaux Athous $\mathrm{du}$ Cancase.

REY (Claudius). Voy. MULSANT.

RITSEMA(Charles). Notes of Leyden museum 1883. (Agrceus constrictus.)

- Entom. Monthl. Mag. 1873-74.

RoJAs (Marc.-Aur. de). Espèces nouvelles du Vénézuéla; in Rev. Zool. 1855.

Rossi (Peter). Fauna Etrusca, 1790. (Elater crucifer.)

- Mantissa insectorum; Spec. in Etruria collecta, 1792-1794.

ROSENHAUER (Will. Gotl.). Beitrage z. insekten fauna Europas (Coléoptères) 1847. (A. mutilatus.)

- Die Thiere Andalusiens; 1856.

RYE (Edw.). The entomological Montley Magaz. III, 1867.

SAHLBERG (John.). Expédition de la Véga; in Midd. Soc. fennica, 1884 (Crypt. barbatus).

Satlti (Auguste). Différentes espèces nouvelles d'Elatérides sont décrites dans : Ann. Soc. entom. France 1854-1855. Revue et Magas. de Zool. 1873, avec figures. (Eud. Boucardi, etc.)

SAXESEN (W.). Isis, 1838 (Cor. Heyeri).

SAY (Thomas). Descriptions of Col. Ins, collect. in the Exped. to the Rocky mountains; in Journ. Acad. Nat. Sc. Philad. III, 1823.

- Descript. of new Amer. spec. of Buprestis, Trachys and Elater; in Ann. Lyceum. New-York 1825.

- Descript. of new N. Americ. spec. in Trans. Amer. Philos. Society VI, 1839. L'auteur décrit dans ces mémoires, nombre d'Elater des Etats-Unis, dont on ne connaissait avant lui que peu de chose.

Schaller (Joh. Gottlob.). New insect. beschrieb.; in Schrift naturg Gesellsch. 1783.

SCHARP (David). Elat. of New Zealand; in Ann, and Magaz. 1877. L'auteur fonde un grand nombre de genres et décrit beaucoup d'espèces de l'Archipel ci-dessus. Il les distribue dans une vingtaine de Groupes en s'affranchissant de toute classifioation antérieure et générale. 
- Monthley Magaz. 1880.

- Transact. R. Acad. Dublin, 1883. Plusieurs Elatérides des îles Sandwich.

SchaUfUSS (L. W.). Isis 1861 (Ath cantabricus). Ibid. 1862 (Elat. aurilegulus). Ibid. 1864 (Alaus basalis).

- Coléoptères nouveaux; in Ann. Soc. Entom. Fr. 1862. (Cardioph. d'Espagne.)

SCHIÖDTE (Joh. Georg.). Kröyer Nat. Tidsskr. 1865.

Scopoli (J. Ant.). Entomologia carniolica, 1763.

ScHRANk (Franz.) Entomol. Beitrage, 1776.

- Enumeratio insectorum Austriæ indigenorum, 1781.

- Bayersch. Reise, 1786.

SeIdlitz (Georg.). Berl. entomol. Zeitschr. 1867 (Dima Perezi).

Serville (J. G. Aucinet). Art. Insectes dans l'Encyclopédie méthodique (avec Lepelletier de St-Fargeau) 1825. (Tetralobus, etc.)

SwIDT (Ferdin. Jos.). Gistel Faunus, 1832. (E. (Adel.) Grafi.)

Solier (Ant. Jos. J.) in Gay Historia fisica y politica de Chile. Zool. V, 1850. Beaucoup de genres et d'espèces chiliennes; les premiers, établis sur des caractères insuffisants.

SolSKI (S.). Hor. Societ. entomol. Rossicæ. Plusieurs articles, dans les années 1871, 1881, 1882, renferment des descriptions d'Elatérides nouveaux.

Sternheil (Edotard). Atti della Soc. Italiana de Scienze natural. Milan, 1873. Elatérides de la Rép. Argentine, dont 9 nouveaux.

- Coléopt. de la N1le Grenade; in Col. Hefte XIV, 1875. (105 Elatérides, dont 50 nouveaux.)

- N. Columb. Elateriden aus Ocania; in Munch. Verein 1877.

Stephexs (James Francis). Illustrat. of British Entomology; vol. VI, 1836-37.

- A manual of brit. Coleopt. 1839.

Steven (Christian von). Description de l'Elater Parreyssi, etc.; in Bull, de Moscou, II, 1830. 
STIERLIN (G.). Mittheil. Schw. Gesellsch. 1862 et années suivantes. Esp. nouvelles.

- Berliner Entomol. Zeitschr. 1864.

Sturii (Dr Jacob). Catalogue 1843. Enumère 13266 Coléopt. dont 371 Elatérides.

SUlzER (Joh.Heinrich). Abgekürzte Geschichte der insekten, etc. 1776.

TARNIER (Frédéric). Coléoptères des îles Açores, 1860.

Thomson (C.G.). Skandinaviens coleoptera synoptisk Bearbet. 1857.

Thomson (James). Description de 17 coléoptères; in Revue et Magas. de Zool. 1856, avec figures. (Iphis mortuus, etc.)

THunberG (Carl. Peter.). Novæ insectorum species. Acta Upsal. 1784. VILLA (Antonio). Coleoptera Europæ dup. in Coll. Villæ. Supplément de 1838.

VILLERs (Ch. Jos, de). Caroli Linnei Entomologia, etc. Lyon 1789.

VOET (Jos. Ezech.). Catalogus system. Coleopterorum; 1806.

WALKER (Francis). Ann. nat. Histor. 1858. Col. de Ceylan.

- Natural. Vancouver II, 1866. Les descriptions de Coléoptères faites par Walker sont difficilement reconnaissables et considérées par beaucoup comme non avenues.

WALTt، (Jos.). Verzeischniss d. um Passau Vork Selt. Käfer, etc.

- Beitr. z. Kenntn. Coleopteren der Turkei. Tous deux publiés dans Isis, 1838.

WATERHoUse (Alph.). Proceed. Zool. Soc. London 1888. (Tetr. cribricollis.)

WATERHOUSE (Georg. Rob.). Description of Col. ins. collect. by Darwin in Gallapagos Island; in Ann. nat. Histor. 1845.

WeBER (Fried.). Observationes entomologiæ, etc. Univ. Buchh. Kiel, 1801.

WESTWOOD (John. Obadiah). Ann. and magaz. of nat. Hist. 1848. (E. perpulcher.)

- Cabinet of oriental Entomology 1848. Quelques Elatérides avec belles figures. 
TVHITE (Adam). Descriptions of Coleopt. a. Orthopt. of new. Zealand; in Zool. Voy. Erebus and Terror XI, 1846.

Wiedemann (Christian Rud.). New Käfer von Java, Bengalen, etc.; in Germ. Magazin. II, 1823.

Wollaston (Th. Vernon). Insecta Maderensia, 1854.

- Coll. Canar. Island; in Ann. a. Magaz. 1858-1862.

- Coll. Canar. 1864. Création du G. Coptostethus et El. nouv. des Canaries.

Zetterstedt (Joh. Will.). Acta Holmiæ, 1824.

- Fauna insectorum lapponica, 1828.

- Insecta lapponica descripta, 1840.

Ziegler (Daniel). Descript. of new N. Amer. Coleopt.; in Proceed. Acad. Philad. 1844. 
TABLE ALPHABÉTIQUE

\section{DES TRIBUS \& DES GENRES}

ÉNUMÉRÉS DANS CE CATALOGUE.

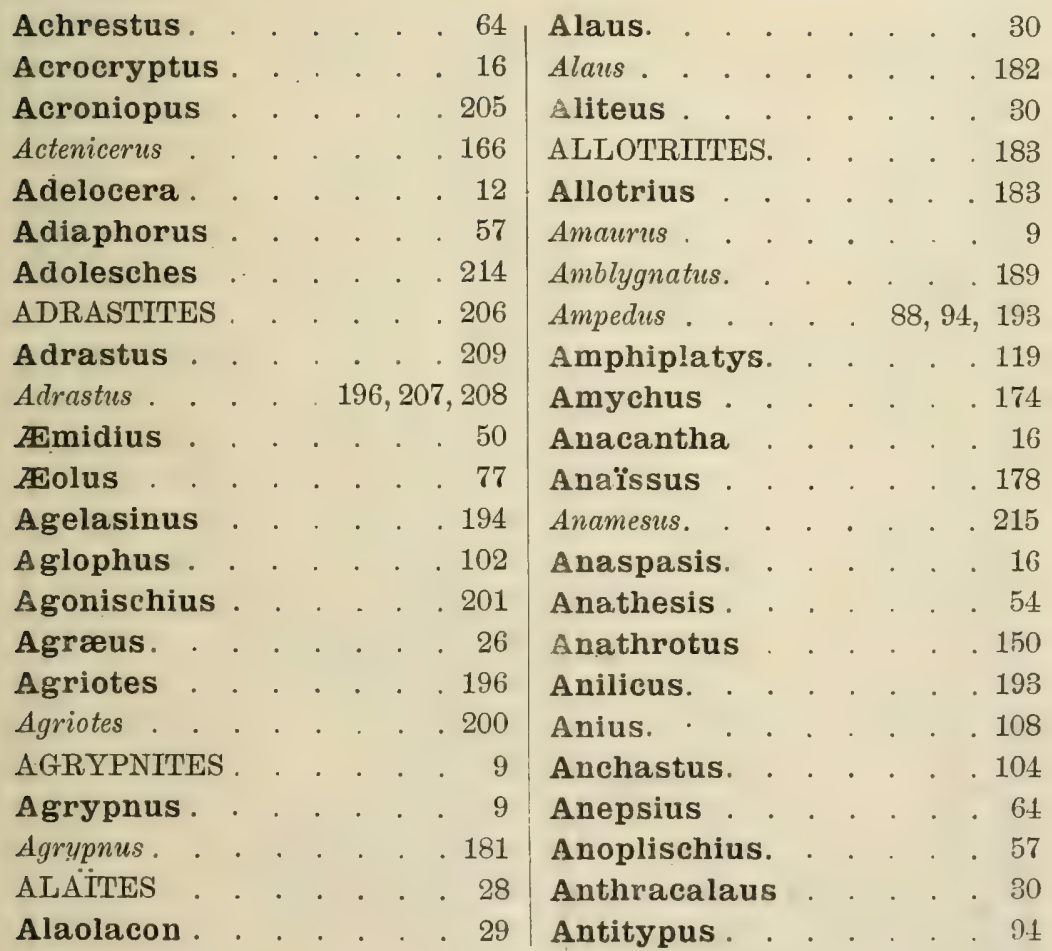




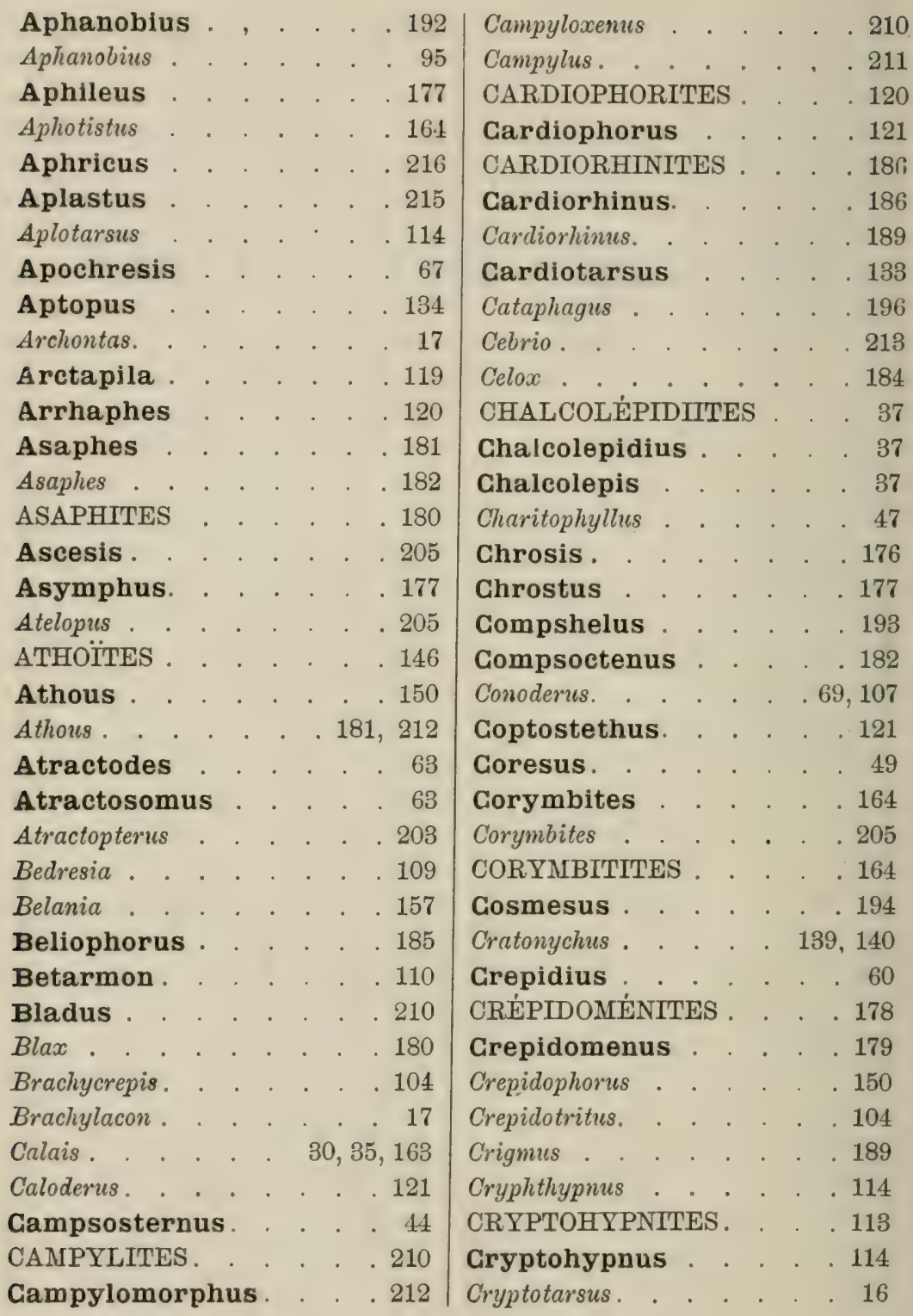




\begin{tabular}{|c|c|c|c|c|c|c|}
\hline & & & & & & \\
\hline tenicera. & . & : 164 & Eopenthes. & & & \\
\hline tenonychus & & 140,207 & xus. & & & 1 \\
\hline tenoplus & . & 209 & Est & & & \\
\hline a & & 57 & Euc & & & \\
\hline rus & & 213 & Enc & & & \\
\hline . & . & 211 & YLITES . & & & \\
\hline & & 108 & & & & \\
\hline & 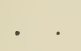 & 164,181 & 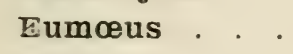 & & & \\
\hline & & 185 & & & & \\
\hline IES & & 49 & us. & & & 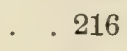 \\
\hline & & 64 & is. & & & 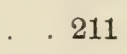 \\
\hline & & . 53 & (a) & & & 14 \\
\hline$i c$ & . & 54,121 & Gar & & & $x^{\prime}$ \\
\hline rus. & . & .205 & Ger & & & 10 \\
\hline & & 15 & $\mathrm{Ge}$ & & & 20 \\
\hline & & 184 & & & & \\
\hline 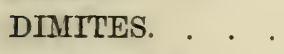 & & 184 & ilus. & & & \\
\hline is . & & . . 192 & & & & 20 \\
\hline & & . . 139 & rus & & & 87 \\
\hline & & 57 & $G r$ & & & 150 \\
\hline & & 103 & Ha & . & & 21 \\
\hline & & .203 & us & & & 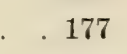 \\
\hline 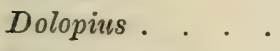 & & . . 196 & $\mathrm{H}_{a}$ & & & 106 \\
\hline le & & . . 204 & $\mathrm{He}$ & & & $16:$ \\
\hline $\operatorname{orcc}$ & & 177 & $\mathrm{He}$ & & & 17 \\
\hline 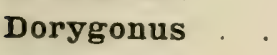 & & & $\mathrm{Hem}$ & & & 18 \\
\hline 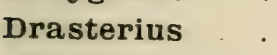 & & 86 & rus & & & 18 \\
\hline tin & .196 & 203,204 & $\mathrm{He}$ & & & 21 \\
\hline & & . . 17 & $\mathrm{He}$ & & & 21 \\
\hline u & & 56 & $\mathrm{He}$ & & & 21 \\
\hline $\operatorname{sem}$ & & 47 & $\mathrm{He}$ & & & 35 \\
\hline & & 88 & $\mathrm{He}$ & & & 120 \\
\hline & & 88 & $\mathrm{He}$ & & & \\
\hline & & 88 & $\mathrm{He}$ & & & \\
\hline & & . 149 & & & & 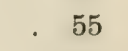 \\
\hline & & & Hexaulacus & & & \\
\hline
\end{tabular}


Hifo

Homotechnes

Horistonotus

Hypnoidus

Hypodesis.

HYPODÉSITES

Hypolithus

Hypsiophthalmus

Ictis

Iphis

Ischiodontus .

Ischiodontus .

Ischius .

Ischnodes .

Isidus

Isosoma

Itodacnus .

Lacon

Leptophyllus

Leptoschema

Lepturoides .

Limonius

Liotrichus

Loboderus

Lomemus .

Ludigenus.

LUDHTES

Ludius

Ludius.

Lycoreus

Macromalocera

Mecastrus.

Mecothorax

Medonia

Megacnemius.

Megalorhipis .

Megapenthes
162

94

135

114

185

185

114

157

212

29

60

50

163

95

149

215

178

17

47

200

211

146

166

59

102

192

187

189

9, 205

$95,164,179,205$
29

212

188

108

109

187

47

95
Melanactes . . . . . 175

MÉLANOTITES . . . - . 139

Melanotus. . . . . 140

Melanoxanthus. . . . 99

Melanoxanthus . . . . . . 193

Melantho . . . . . . 179

Melanthoides . . . . $6 ;$;

Meristhus. . . . . . 26

Meroplinthus . . . . 163

Metablax . . . . . 180

Monadicus . . . . 114

Monelasmus . . . . . . 193

MONOCRÉPIDHTES . . . 67

Monocrepidius . . . . 68

Morostoma . . . . . . 183

Mivrmodes . . . . . 27

Negastrius . . . . . . . 114

Nomophleus . . . . . 212

Nothodes . . . . . . 174

Nycterilampus . . . . . 205

Ochosternus . . . . . 205

Coneus . . . . . . . 17

Octinodes . . . . . . . 213

Oedostethus . . . . . . 120

Oestodes . . . . . . 210

Oistus . . . . . 44

Olophoeus. . . . . 50

Ophidius . . . . . . 180

Ophidius . . . . . . 193

Optaleus . . . . . . . 17

Orithales. . . . . . 166

Orthostethus. . . . . . 192

Osorno . . . 185

Ovipalpus . . . . . . 59

Oxycleidins . . . . . 57

Oxygonus . . . . . . 200

oxylasma . . . . . 178 
OXYNOPTÉRITES . . . . 46

Oxynopterus. . . . . 46

Oxysternus . . . . . . . 210

Oxystethus . . . . . 102

Pachyderes . . . . . 66

Panspæus. . . . . . . 111

Pantolamprus . . . . 50

Parallotrius . . . . . 183

Paranilicus . . . . . 193

Paranomus . . . . . . 174

Paraphileus . . . . . . 163

Parasaphes . . . . 182

Parelater. . . . . . . 94

Parhemiops . . . . . 214

Parinus. . . . . . . 178

Pectocera. . . . . . 47

Pedetes. . . . . . . 150

Penia. . . . . . . . 184

Pericallus. . . . . . . . 40

Pericus. . . . . . . 27

Perimecus. . . . . . . 140

Phanophorus . . . . 210

Phanophorus. . . . . . $15 \pi$

Phedomenus . . . . 68

Pheletes . . . , . . 136

Phorotarsus . . . . . . . 120

Photophorus . . . . 162

Phyllophorus. . . . . . . 47

PHYSORHINITHS . . . . 103

Physorhinus . . . . 103

Piezophyllus. . . . . . 49

Pittonotus. . . . . . 204

Pityobius . . . . . . 149

Platynychus . . . . . 121

PLASTOCÉRITES . . . . 215

Plastocerus . . . . . 215

Plectrosternus
Pleonomus . . . . . 212

Podeonius. . . . . . 104

Podonema. . . . . . . 108

POMACHILHTES. . . . 107

Pomachilius . . . . . . 111

Pomatochilus. . . . . . 111

Porthmidius . . . . . 104

Priopus . . . . . . 140

Pristilophus . . . . . 174

Pristilophus . . . . . . 164

Probotrium . . . . . 188

Prosternon . . . . . 164

Protelater. . . . . . 110

Psellis . . . . . . . 139

Psephus . . . . . 50

Pseudreolus . . . . . . . 77

Pseudiconus . . . . . 120

Psiloniscus . . . . . 107

Psorochroa . . . . . 178

Pyrapractes. . . . . 164

PYROPHORITES. . . . 157

Pyrophorus . . . . . . 157

Scaphoderus. . . . . . 12

Scelisus. . . . . . . 192

Scoliocerus . . . . . . 114

Selatosomus . . . . . . 164

Semiotus . . . . . 40

Semiotopsis . . . . . . 44

Senodonia. . . . . . 183

Sephilus . . . . . . 55

Sericosomus . . . . . . 203

Sericus . . . . . 203

Silesis . . . . . 208

Simodactylus . . . . 65

Singhalenus . . . . . 56

Smilicerus. . . . . . 111

Sphenomerus . . . . 56 


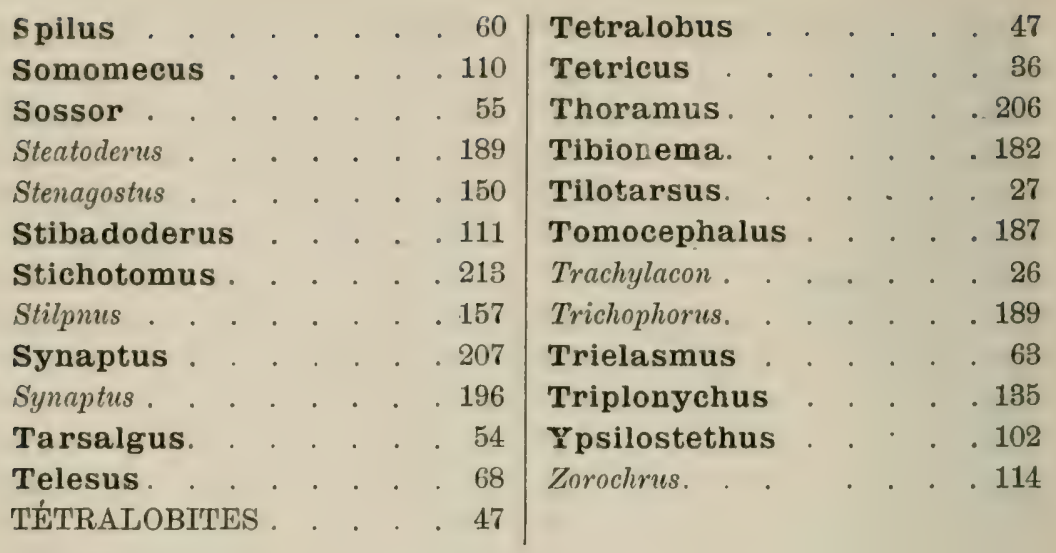












\section{型}

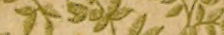

$9=-32,-x$

(5)

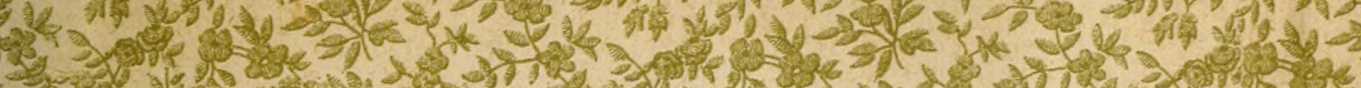

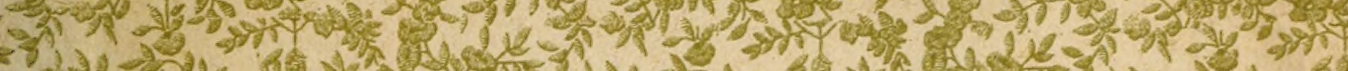
- 4 1. a) 2,2 ,

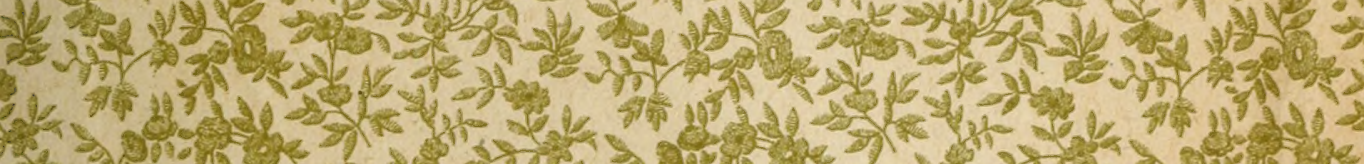

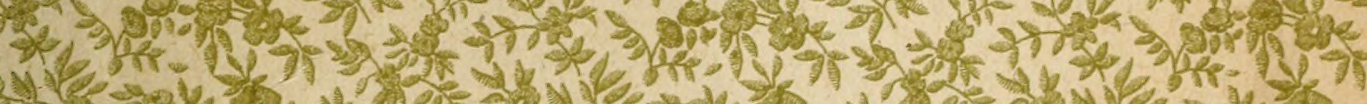

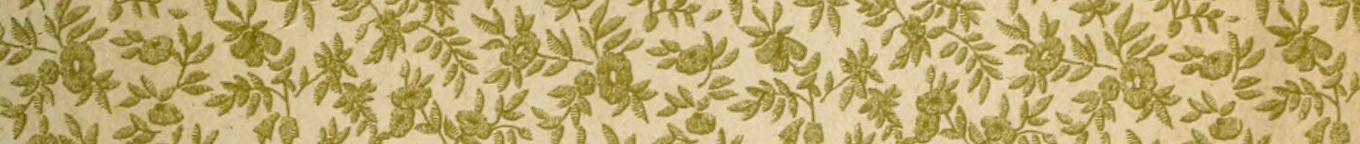

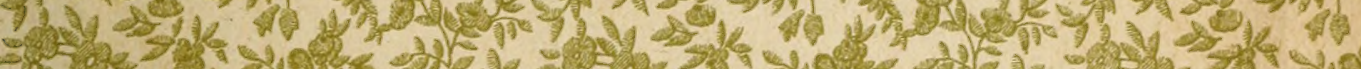
Fo 24.

(1) 2. 1) 320

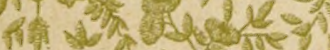
1. 1)

\section{res 2} Le $20.72 \%$ 1 . 10 . $\sqrt{3}$

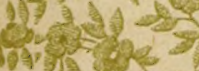
t?

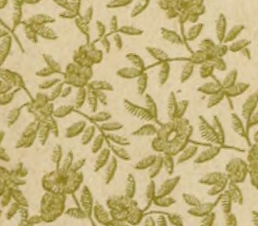
(1) $(-3)$

$5>3$ al 2 की $21-25$

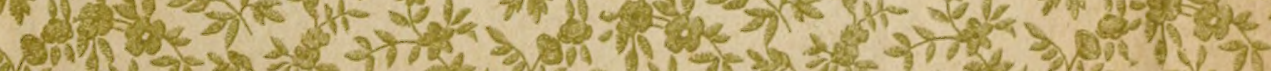

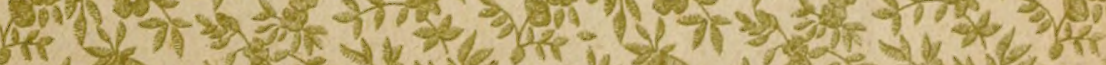

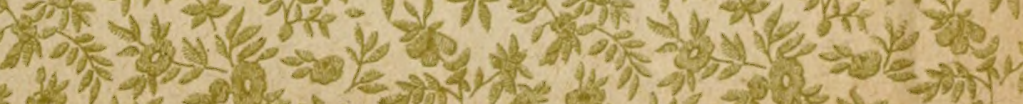


